\title{
Patient-specific cardiovascular modeling in pulmonary hypertension : assessment of hemodynamic and mechanical ventricular interaction
}

Citation for published version (APA):

Lumens, J. E. (2010). Patient-specific cardiovascular modeling in pulmonary hypertension : assessment of hemodynamic and mechanical ventricular interaction. [Doctoral Thesis, Maastricht University]. dlgraphics. https://doi.org/10.26481/dis.20100702jl

Document status and date:

Published: 01/01/2010

DOI:

10.26481/dis.20100702jl

Document Version:

Publisher's PDF, also known as Version of record

Please check the document version of this publication:

- A submitted manuscript is the version of the article upon submission and before peer-review. There can be important differences between the submitted version and the official published version of record.

People interested in the research are advised to contact the author for the final version of the publication, or visit the DOI to the publisher's website.

- The final author version and the galley proof are versions of the publication after peer review.

- The final published version features the final layout of the paper including the volume, issue and page numbers.

Link to publication

\footnotetext{
General rights rights.

- You may freely distribute the URL identifying the publication in the public portal. please follow below link for the End User Agreement:

www.umlib.nl/taverne-license

Take down policy

If you believe that this document breaches copyright please contact us at:

repository@maastrichtuniversity.nl

providing details and we will investigate your claim.
}

Copyright and moral rights for the publications made accessible in the public portal are retained by the authors and/or other copyright owners and it is a condition of accessing publications that users recognise and abide by the legal requirements associated with these

- Users may download and print one copy of any publication from the public portal for the purpose of private study or research.

- You may not further distribute the material or use it for any profit-making activity or commercial gain

If the publication is distributed under the terms of Article $25 \mathrm{fa}$ of the Dutch Copyright Act, indicated by the "Taverne" license above, 
Patient-Specific Cardiovascular Modeling

in Pulmonary Hypertension

Assessment of Hemodynamic and

Mechanical Ventricular Interaction

Joost Lumens 



\section{Patient-Specific Cardiovascular Modeling in Pulmonary Hypertension Assessment of Hemodynamic and Mechanical Ventricular Interaction}

\section{PROEFSCHRIFT}

ter verkrijging van de graad van doctor aan de Universiteit Maastricht, op gezag van de Rector Magnificus, Prof. mr. G.P.M.F. Mols, volgens het besluit van het College van Decanen in het openbaar te verdedigen op vrijdag 2 juli 2010 om 14:00 uur

door

\section{Jozef Elisabeth Lumens}

geboren op 28 mei 1982

te Sittard 


\section{Promotores:}

Prof. dr. ir. T. Arts

Prof. dr. T. Delhaas

\section{Beoordelingscommissie:}

Prof. dr. H.P. Brunner-La Rocca (voorzitter)

Prof. dr. R. Naeije (Free University of Brussels)

Prof. dr. L.H.E.H. Snoeckx

Prof. dr. J.V. Tyberg (University of Calgary)

Prof. dr. ir. F.N. van de Vosse

This research project has been financially supported by an unrestricted grant from Actelion Pharmaceuticals Nederland BV, Woerden, The Netherlands.

Financial support by the Netherlands Heart Foundation and Stichting Hartsvrienden RESCAR for the publication of this thesis is gratefully acknowledged. 


\section{Wijze boeken}

als ik die dikke boeken zie van de geleerden

vol wijze dingen die zij allemaal beweerden en ik zie de wereld om me heen, dan moet ik vrezen

dat niemand ooit die wijze boeken heeft gelezen

\section{Toon Hermans}


ISBN/EAN:

978-90-8590-860-9

Cover:

Ontwerp: Joost Lumens

Uitvoering: Eric Lemmens

Lay-out:

Babs Grit / Eric Lemmens - D\&L graphics

Pulished by:

www.dlgraphics.nl

Printed by:

Schrijen-Lippertz 


\section{TABLE OF CONTENTS}

Chapter 1

General Introduction

Chapter 2

Cardiac Anatomy and Physiology Under Normal and Pulmonary

Hypertensive Conditions

\section{Chapter 3}

Modeling Ventricular Interaction: a Multiscale Approach from Sarcomere Mechanics to Cardiovascular System Hemodynamics

\section{Chapter 4}

Three-Wall Segment (TriSeg) Model Describing Mechanics and Hemodynamics of Ventricular Interaction.

\section{Chapter 5}

Right Ventricular Free Wall Pacing Improves Cardiac Pump Function in Severe Pulmonary Arterial Hypertension: a Computer Simulation Analysis.

\section{Chapter 6}

Left Ventricular Underfilling and Not Septal Bulging Dominates Abnormal Left Ventricular Hemodynamics in Chronic Thromboembolic Pulmonary Hypertension.

\section{Chapter 7}

Patient-Specific Modeling for Assessment of Ventricular Mechanical Asynchrony in Pulmonary Arterial Hypertension

\section{Chapter 8}

General Discussion

Summary

Samenvatting

Dankwoord

Curriculum Vitae

List of Publications 



\section{Chapter'}

General Introduction 
Chapter 1 


\section{General Introduction}

Pulmonary hypertension $(\mathrm{PH})$ is a pathological condition that is characterized by elevated blood pressure in the pulmonary artery. PH has been defined clinically as a mean pulmonary artery pressure, measured by right heart catheterization, of $>25 \mathrm{mmHg}$ at rest, or $>30 \mathrm{mmHg}$ with exercise. ${ }^{1}$ The most recent clinical classification of PH (Dana Point classification) distinguishes five subgroups. ${ }^{39}$ Each subgroup of PH shares pathological and clinical features as well as similar therapeutic options. In this thesis, we focus on two subgroups, i.e., pulmonary arterial hypertension (PAH) and chronic thromboembolic pulmonary hypertension (CTEPH). PAH is defined as a group of diseases with a common characteristic histopathological pattern of pulmonary vascular remodeling. This vascular remodeling results in resistive lesions in the pulmonary vascular bed progressively leading to chronic elevation of pulmonary arterial pressure. ${ }^{16,38}$ $\mathrm{PAH}$ is a rare disease as evidenced by low estimates of prevalence and incidence, i.e., 15.0 cases per million of adult inhabitants and 2.4 cases per million of adult inhabitants per year, respectively. ${ }^{22}$ Furthermore, CTEPH is a late-occurring complication of pulmonary embolism. ${ }^{3,}{ }^{34} \mathrm{CTEPH}$ can be considered as a type of $\mathrm{PAH}$, since it also progressively develops due to similar histopathological changes in the pulmonary vasculature as seen in other forms of severe nonthromboembolic PAH. ${ }^{11}$, 15, 32 In the remainder of this thesis, the term PAH also includes CTEPH, unless stated differently.

When left untreated, PAH progressively leads to right heart failure and premature death within 2.8 years from diagnosis. ${ }^{9,} 29,38$ Several studies showed that morbidity and mortality of PAH patients are determined by the ability of the right ventricle (RV) to maintain normal stroke volume and cardiac output despite pressure overload. 8, 31, 32, 35, 36

Historically, knowledge of RV pump function under normal as well as under pathological conditions has lagged behind that of the left ventricle (LV). ${ }^{17}$ Consequently, the mechanisms of RV failure are poorly understood. Over the last decade, however, the RV is gaining interest, partly driven by the insight that RV performance is of pivotal importance in patients with PAH. $8,17,19,20,28,41$ The $\mathrm{LV}$ and the RV are functionally dependent due to their mechanical interaction via the interventricular septum and their hemodynamic series interaction via the pulmonary and systemic circulations. 2, 4-6, 10, 13, 14, 23, 25, 33, 37, 40, 42 Thus, in daily clinical practice, understanding the mechanisms of ventricular interaction is also essential for proper treatment of right heart failure in $\mathrm{PAH}$. 


\section{General Aim of the Thesis}

In this thesis, we aim to elucidate the mechanisms by which PAH affects left and right ventricular pump function. For that purpose, we developed a mathematical model of cardiovascular mechanics incorporating hemodynamic and mechanical ventricular interaction. This model was used for hypothesis-driven investigation of the effect of PAH on cardiac pump function. Each of these investigations starts with fitting the model to the average of a set of clinical measurements obtained in patients with PAH in order to obtain a representative pathologyspecific simulation of ventricular mechanics and hemodynamics as function of time. Using the pathology-specific simulation as point of departure, the model was used 1) to test pacing of the RV free wall for its potential to treat RV failure in patients with severe PAH, 2) to quantify contributions of mechanical and hemodynamic ventricular interaction to LV filling hemodynamics in patients with CTEPH before and after surgically induced decrease of pulmonary vascular resistance, and 3) to assess ventricular mechanics patient-specifically for better understanding of ventricular mechanical asynchrony in the individual PAH patient.

\section{OUtLine of the Thesis}

The thesis starts with an introduction to relevant aspects of cardiac anatomy and physiology in chapter 2. The influence of PAH on cardiac anatomy and physiology is also addressed in this chapter.

Chapters 3 and 4 comprise the mathematical basis of a new three-wall segment model (TriSeg model) describing ventricular mechanics and hemodynamics incorporating mechanical interaction of the LV free wall, the interventricular septum, and the RV free wall.

In chapter 3, the TriSeg model is introduced and used to simulate ventricular mechanics and hemodynamics with normal synchronous and with abnormal asynchronous mechanical activation of the ventricular walls. Simulation results of ventricular geometries and pressures are compared with experimental data obtained in dogs before and after acute induction of left bundle branch block, i.e., delayed onset of mechanical activation of the LV free wall compared to that of the interventricular septum and RV free wall.

The theory of the TriSeg model is described elaborately in chapter 4 . The model is used to simulate cardiac mechanics and hemodynamics with normal 
mechanical loading, with acute increase of pulmonary vascular resistance, and with increase of pulmonary vascular resistance including adaptation of the ventricular walls to increased mechanical load. Simulation results are compared with clinical data on ventricular mechanics and hemodynamics as obtained in healthy volunteers and in patients with $\mathrm{PAH}$. Furthermore, the relation between geometry of the interventricular septum and its transmural pressure difference, as simulated, is compared with this relation as measured in patients with and without PAH.

In the second part of the thesis (chapters 5-7), the model is used to obtain a better understanding of the cardiac pathophysiology in PAH and to quantify the effect of therapeutic interventions on cardiac pump function.

In PAH, duration of myofiber shortening is prolonged in the RV free wall as compared to that in the interventricular septum and LV free wall. ${ }^{27}$ This ventricular mechanical asynchrony eventually leads to right heart failure. It has also been shown that PAH is associated with electrophysiological changes in the $\mathrm{RV}$ free wall, i.e., conduction slowing and action potential prolongation. 7, 21, 24 We used the model to explore whether early pacing of the RV free wall may improve interventricular mechanical synchrony and, hence, cardiac pump function in patients with severe PAH (chapter 5).

In patients with CTEPH, chronic increase of RV afterload is associated with an abnormal LV filling pattern, 12, 18, 26, 30 i.e., early diastolic peak of mitral blood flow velocity $(E)$ is smaller than the peak flow velocity related to atrial contraction (A). Pulmonary thromboendarterectomy is a surgical intervention that acutely reduces pulmonary vascular resistance and is associated with acute increase of mitral E/A-ratio. ${ }^{18,26,30}$ Resolution of leftward septal bulging and increase in RV systolic output are thought to be responsible for the increase of the E/A-ratio. The TriSeg model is used to quantify the separate contributions of leftward septal bulging and of RV systolic output to LV filling hemodynamics in a group of CTEPH patients before and after pulmonary thromboendarterectomy (chapter 6).

A patient specific simulation approach is designed to assess the mechanism of ventricular mechanical asynchrony in the individual patient with PAH (chapter 7).

The thesis ends with chapter 8 , a general discussion linking the findings of abovementioned studies and putting them in a broader perspective. Potential clinical implications are discussed and are followed by suggestions for related future studies. 
Chapter 1 


\section{REFERENCES}

1. Badesch DB, Champion HC, Sanchez MA, Hoeper MM, Loyd JE, Manes A, McGoon M, Naeije R, Olschewski H, Oudiz RJ, and Torbicki A. Diagnosis and assessment of pulmonary arterial hypertension. J Am Coll Cardiol 54: S55-66, 2009.

2. Baker AE, Dani R, Smith ER, Tyberg JV, and Belenkie I. Quantitative assessment of independent contributions of pericardium and septum to direct ventricular interaction. Am J Physiol 275: H476-483, 1998.

3. Becattini C, Agnelli G, Pesavento R, Silingardi M, Poggio R, Taliani MR, and Ageno W. Incidence of chronic thromboembolic pulmonary hypertension after a first episode of pulmonary embolism. Chest 130: 172-175, 2006.

4. Beyar R, Dong SJ, Smith ER, Belenkie I, and Tyberg JV. Ventricular interaction and septal deformation: A model compared with experimental data. Am J Physiol 265: H20442056, 1993.

5. Beyar R, Hausknecht MJ, Halperin HR, Yin FC, and Weisfeldt ML. Interaction between cardiac chambers and thoracic pressure in intact circulation. Am J Physiol 253: H12401252, 1987.

6. Brinker JA, Weiss JL, Lappe DL, Rabson JL, Summer WR, Permutt S, and Weisfeldt ML. Leftward septal displacement during right ventricular loading in man. Circulation 61: 626-633, 1980.

7. Chen PS, Moser KM, Dembitsky WP, Auger WR, Daily PO, Calisi CM, Jamieson SW, and Feld GK. Epicardial activation and repolarization patterns in patients with right ventricular hypertrophy. Circulation 83: 104-118, 1991.

8. Chin KM, Kim NH, and Rubin LJ. The right ventricle in pulmonary hypertension. Coron Artery Dis 16: 13-18, 2005.

9. D'Alonzo GE, Barst RJ, Ayres SM, Bergofsky EH, Brundage BH, Detre KM, Fishman AP, Goldring RM, Groves BM, Kernis JT, and et al. Survival in patients with primary pulmonary hypertension. Results from a national prospective registry. Ann Intern Med 115: 343-349, 1991.

10. Damiano RJ, Jr., La Follette P, Jr., Cox JL, Lowe JE, and Santamore WP. Significant left ventricular contribution to right ventricular systolic function. Am J Physiol 261: H15141524, 1991.

11. Dartevelle P, Fadel E, Mussot S, Chapelier A, Herve P, de Perrot M, Cerrina J, Ladurie FL, Lehouerou D, Humbert M, Sitbon O, and Simonneau G. Chronic thromboembolic pulmonary hypertension. Eur Respir J 23: 637-648, 2004.

12. Dittrich HC, Chow LC, and Nicod PH. Early improvement in left ventricular diastolic function after relief of chronic right ventricular pressure overload. Circulation 80: 823830, 1989. 


\section{Chapter 1}

13. Dong SJ, Smith ER, and Tyberg JV. Changes in the radius of curvature of the ventricular septum at end diastole during pulmonary arterial and aortic constrictions in the dog. Circulation 86: 1280-1290, 1992.

14. Elzinga G, van Grondelle R, Westerhof N, and van den Bos GC. Ventricular interference. Am J Physiol 226: 941-947, 1974.

15. Galie N, and Kim NH. Pulmonary microvascular disease in chronic thromboembolic pulmonary hypertension. Proc Am Thorac Soc 3: 571-576, 2006.

16. Galie N, Torbicki A, Barst R, Dartevelle P, Haworth S, Higenbottam T, Olschewski H, Peacock A, Pietra G, Rubin LJ, Simonneau G, Priori SG, Garcia MA, Blanc JJ, Budaj A, Cowie M, Dean V, Deckers J, Burgos EF, Lekakis J, Lindahl B, Mazzotta G, McGregor K, Morais J, Oto A, Smiseth OA, Barbera JA, Gibbs S, Hoeper M, Humbert M, Naeije R, and Pepke-Zaba J. Guidelines on diagnosis and treatment of pulmonary arterial hypertension. The task force on diagnosis and treatment of pulmonary arterial hypertension of the european society of cardiology. Eur Heart J 25: 2243-2278, 2004.

17. Greyson CR. Pathophysiology of right ventricular failure. Crit Care Med 36: S57-65, 2008.

18. Gurudevan SV, Malouf PJ, Auger WR, Waltman TJ, Madani M, Raisinghani AB, DeMaria AN, and Blanchard DG. Abnormal left ventricular diastolic filling in chronic thromboembolic pulmonary hypertension: True diastolic dysfunction or left ventricular underfilling? J Am Coll Cardiol 49: 1334-1339, 2007.

19. Haddad F, Doyle R, Murphy DJ, and Hunt SA. Right ventricular function in cardiovascular disease, part ii: Pathophysiology, clinical importance, and management of right ventricular failure. Circulation 117: 1717-1731, 2008.

20. Haddad F, Hunt SA, Rosenthal DN, and Murphy DJ. Right ventricular function in cardiovascular disease, part i: Anatomy, physiology, aging, and functional assessment of the right ventricle. Circulation 117: 1436-1448, 2008.

21. Hardziyenka M, Campian ME, Bouma BJ, Linnenbank AC, de Bruin-Bon HA, Kloek JJ, van der Wal AC, Baan J, Jr., de Beaumont EM, Reesink HJ, de Bakker JM, Bresser P, and Tan HL. Right-to-left ventricular diastolic delay in chronic thromboembolic pulmonary hypertension is associated with activation delay and action potential prolongation in right ventricle. Circ Arrhythm Electrophysiol 2: 555-561, 2009.

22. Humbert M, Sitbon O, Chaouat A, Bertocchi M, Habib G, Gressin V, Yaici A, Weitzenblum E, Cordier JF, Chabot F, Dromer C, Pison C, Reynaud-Gaubert M, Haloun A, Laurent M, Hachulla $E$, and Simonneau G. Pulmonary arterial hypertension in france: Results from a national registry. Am J Respir Crit Care Med 173: 1023-1030, 2006.

23. Janicki JS, and Weber KT. The pericardium and ventricular interaction, distensibility, and function. Am J Physiol 238: H494-503, 1980. 
24. Li GR, Lau CP, Leung TK, and Nattel S. Ionic current abnormalities associated with prolonged action potentials in cardiomyocytes from diseased human right ventricles. Heart Rhythm 1: 460-468, 2004.

25. Little WC, Badke FR, and O'Rourke RA. Effect of right ventricular pressure on the enddiastolic left ventricular pressure-volume relationship before and after chronic right ventricular pressure overload in dogs without pericardia. Circ Res 54: 719-730, 1984.

26. Mahmud E, Raisinghani A, Hassankhani A, Sadeghi HM, Strachan GM, Auger W, DeMaria $A N$, and Blanchard DG. Correlation of left ventricular diastolic filling characteristics with right ventricular overload and pulmonary artery pressure in chronic thromboembolic pulmonary hypertension. J Am Coll Cardiol 40: 318-324, 2002.

27. Marcus JT, Gan CT, Zwanenburg JJ, Boonstra A, Allaart CP, Gotte MJ, and VonkNoordegraaf A. Interventricular mechanical asynchrony in pulmonary arterial hypertension: Left-to-right delay in peak shortening is related to right ventricular overload and left ventricular underfilling. J Am Coll Cardiol 51: 750-757, 2008.

28. McDonald MA, and Ross HJ. Trying to succeed when the right ventricle fails. Curr Opin Cardiol 24: 239-245, 2009.

29. McLaughlin VV. Survival in patients with pulmonary arterial hypertension treated with first-line bosentan. Eur J Clin Invest 36 Suppl 3: 10-15, 2006.

30. Menzel T, Wagner S, Kramm T, Mohr-Kahaly S, Mayer E, Braeuninger S, and Meyer J. Pathophysiology of impaired right and left ventricular function in chronic embolic pulmonary hypertension: Changes after pulmonary thromboendarterectomy. Chest 118 : 897-903, 2000.

31. Moser KM, Auger WR, and Fedullo PF. Chronic major-vessel thromboembolic pulmonary hypertension. Circulation 81: 1735-1743, 1990.

32. Moser KM, and Bloor CM. Pulmonary vascular lesions occurring in patients with chronic major vessel thromboembolic pulmonary hypertension. Chest 103: 685-692, 1993.

33. Olsen CO, Tyson GS, Maier GW, Spratt JA, Davis JW, and Rankin JS. Dynamic ventricular interaction in the conscious dog. Circ Res 52: 85-104, 1983.

34. Pengo V, Lensing AW, Prins MH, Marchiori A, Davidson BL, Tiozzo F, Albanese P, Biasiolo A, Pegoraro C, lliceto $S$, and Prandoni P. Incidence of chronic thromboembolic pulmonary hypertension after pulmonary embolism. N Engl J Med 350: 2257-2264, 2004.

35. Riedel M, Stanek V, Widimsky J, and Prerovsky I. Longterm follow-up of patients with pulmonary thromboembolism. Late prognosis and evolution of hemodynamic and respiratory data. Chest 81: 151-158, 1982.

36. Sandoval J, Bauerle O, Palomar A, Gomez A, Martinez-Guerra ML, Beltran M, and Guerrero ML. Survival in primary pulmonary hypertension. Validation of a prognostic equation. Circulation 89: 1733-1744, 1994. 
37. Santamore WP, Shaffer T, and Hughes D. A theoretical and experimental model of ventricular interdependence. Basic Res Cardiol 81: 529-538, 1986.

38. Simonneau G, Galie N, Rubin LJ, Langleben D, Seeger W, Domenighetti G, Gibbs S, Lebrec D, Speich R, Beghetti M, Rich S, and Fishman A. Clinical classification of pulmonary hypertension. J Am Coll Cardiol 43: 5S-12S, 2004.

39. Simonneau G, Robbins IM, Beghetti M, Channick RN, Delcroix M, Denton CP, Elliott CG, Gaine SP, Gladwin MT, Jing ZC, Krowka MJ, Langleben D, Nakanishi N, and Souza R. Updated clinical classification of pulmonary hypertension. J Am Coll Cardiol 54: S43-54, 2009.

40. Slinker BK, and Glantz SA. End-systolic and end-diastolic ventricular interaction. Am J Physiol 251: H1062-1075, 1986.

41. Voelkel NF, Quaife RA, Leinwand LA, Barst RJ, McGoon MD, Meldrum DR, Dupuis J, Long CS, Rubin LJ, Smart FW, Suzuki YJ, Gladwin M, Denholm EM, and Gail DB. Right ventricular function and failure: Report of a national heart, lung, and blood institute working group on cellular and molecular mechanisms of right heart failure. Circulation 114: 1883-1891, 2006.

42. Weber KT, Janicki JS, Shroff S, and Fishman AP. Contractile mechanics and interaction of the right and left ventricles. Am J Cardiol 47: 686-695, 1981. 
Background and General Aim of the Thesis 


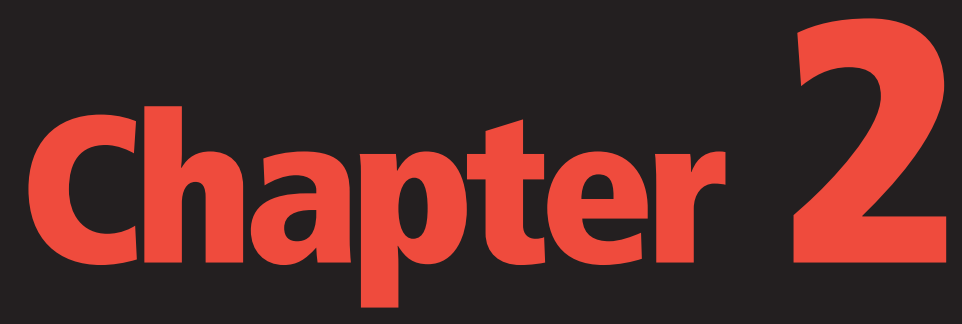

Cardiac Anatomy and Physiology Under Normal and Pulmonary Hypertensive Conditions 


\section{The Heart as a Pump}

The heart is a dual pump that drives the blood in two serial circuits, i.e., the pulmonary and the systemic circulation (Figure 2.1). The pump work needed to achieve blood circulation is delivered by four hollow chambers enclosed by muscular walls (Figure 2.2). Deoxygenated blood returning from the systemic circulation arrives via the coronary sinus and the inferior and superior vena cava in the right atrium. Via the tricuspid valve, the blood then enters the right ventricle (RV). The RV pumps the blood via the pulmonary valve through the main pulmonary artery into the pulmonary peripheral circulation. The pulmonary circulation then provides the blood with oxygen and removes carbon dioxide. Subsequently, the oxygenated blood returns to the heart and arrives in the left atrium via the pulmonary veins. Via the mitral valve, the blood enters the left ventricle (LV). The LV propels the blood via the aortic valve through the aorta into the systemic peripheral circulation, i.e., body organs and tissues. Finally, the blood re-enters the right atrium via the coronary sinus and the venae cavae, thus closing the circulation.

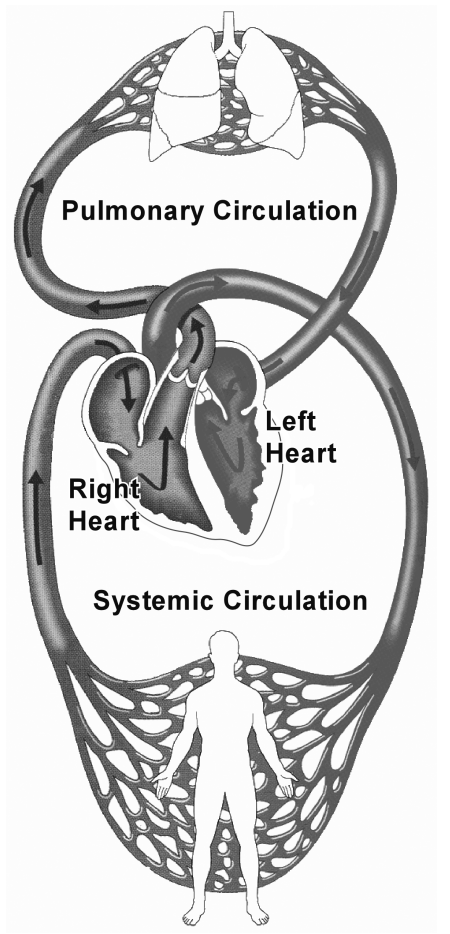

Figure 2.1 Schematic representation of the human cardiovascular system. The pulmonary and the systemic circulation are coupled in series with the heart in between them. The right heart receives deoxygenated blood from the body tissues and pumps it into the pulmonary circulation. After passing the lungs, the oxygenated blood enters the left heart which pumps the blood into the systemic circulation to supply the body tissues. [Adapted from E.N. Marieb, Human Anatomy and Physiology. Fourth Edition. Benjamin Cummings, 1998] 


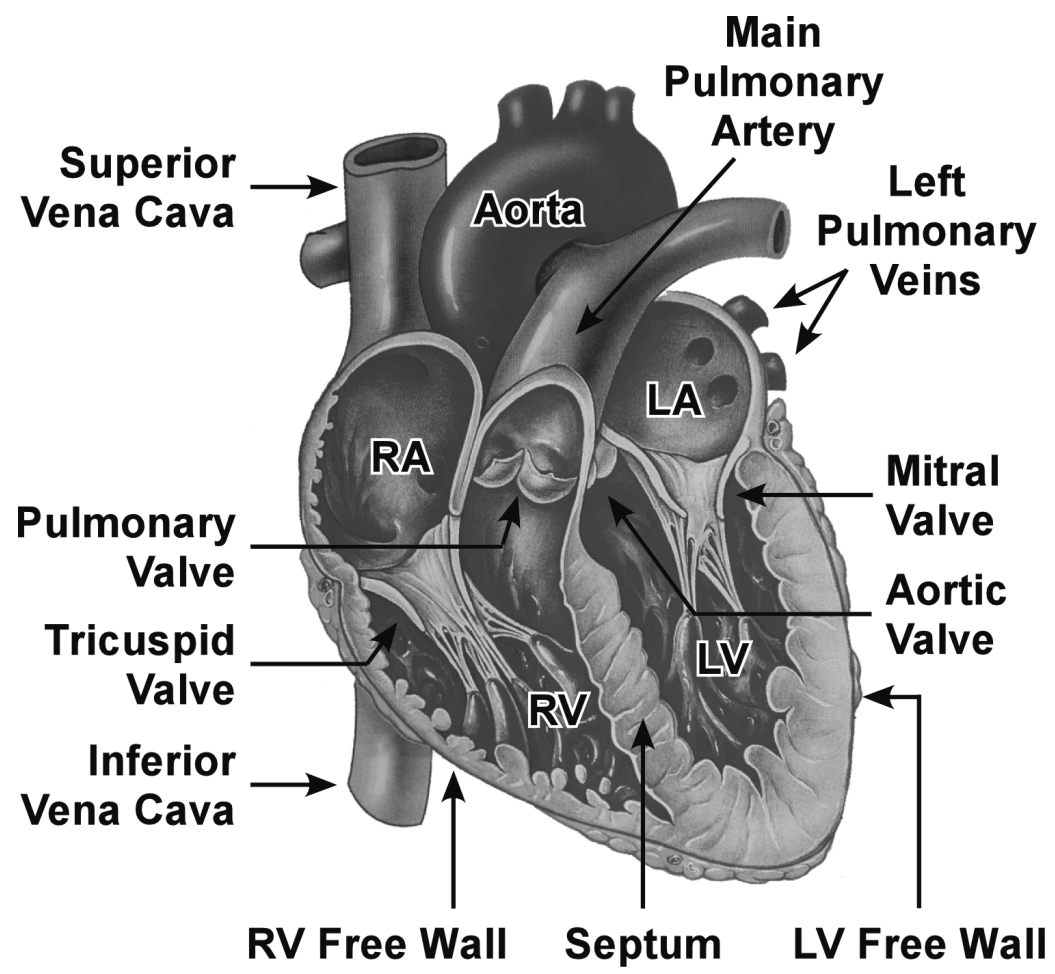

Figure 2.2 Anatomy of the heart. This schematic representation shows a cross-section of the heart along its long axis. Abbreviations: $L A=$ left atrium; $L V=$ left ventricle; $R A=$ right atrium; $R V=$ right ventricle. [Adapted from E.N. Marieb, Human Anatomy and Physiology. Fourth Edition. Benjamin Cummings, 1998]

\section{Structure and Function of the Cardiac Walls}

The myocardial walls of the heart mainly consist of two types of cells, i.e., myocytes and fibroblasts. ${ }^{17,33,34}$ Myocytes are responsible for contraction of the heart. Inside the myocytes, contractile force is generated by contractile units called sarcomeres. Locally, myocytes are more or less aligned to form myofibers. ${ }^{24,61}$ Myocytes are embedded in the extra-cellular matrix, a fibrous network mainly consisting of collagen. The extra-cellular matrix plays an important role in transmitting the contractile force generated by the sarcomeres to the ventricular chamber. Fibroblasts play a pivotal role in the regulation of composition and structure of the extra-cellular matrix. ${ }^{60}$ 


\section{The Cardiac Cycle}

Figure 2.3 shows the time courses of atrial, ventricular, and arterial pressures as well as ventricular volumes and flows through valves during a cardiac cycle of the normal human heart. A cardiac pump cycle is generally divided into the phases of filling, isovolumic contraction, ejection, and isovolumic relaxation. As shown by the time courses of blood flow through the mitral and tricuspid valves (Figure 2.3), the filling phase can be divided in an early diastolic filling phase (E-wave) and a subsequent active filling phase induced by atrial contraction (A-wave). In Figure 2.3, the cardiac cycle starts with atrial contraction and thus with the Awave of ventricular filling and the related increase of ventricular pressure. Next, the myofibers in the ventricular walls are activated. Increase of ventricular pressure beyond atrial pressure causes closure of the atrioventricular valve (mitral or tricuspid valve). During the subsequent isovolumic contraction phase, further mechanical activation of the myofibers in the ventricular walls causes additional increase of ventricular pressure while ventricular volume remains constant with all valves closed. Increase of ventricular pressure beyond arterial pressure causes opening of the ventriculoarterial valve (aortic or pulmonary valve). Subsequently, blood is ejected into the large arteries (aorta and pulmonary artery). At the end of ejection, the myofibers relax and ventricular pressure drops below arterial pressure, causing closure of the ventriculoarterial valve. During the subsequent isovolumic relaxation phase, ventricular pressure drops further while ventricular volume remains constant since all valves are closed. Decrease of ventricular pressure below atrial pressure causes opening of the atrioventricular valve and results in an early diastolic filling wave (E-wave). During the latter passive filling phase, ventricular cavity volume increases rapidly and atrial and ventricular pressures evolve in parallel.

As depicted in Figure 2.3, the shapes of the pressure tracings of the left and right heart are quite similar. However, pressures in the right heart (Figure 2.3A) are about one fifth of those in the left heart (Figure 2.3B). Under hemodynamic steady-state conditions, volume changes of the LV are the same as those of the RV because both ventricles eject the same amount of blood. Despite small left-to-right differences in timing of valve opening and closure, hemodynamic tracings show relative left-right synchrony of contractions and associated hemody-namics. 

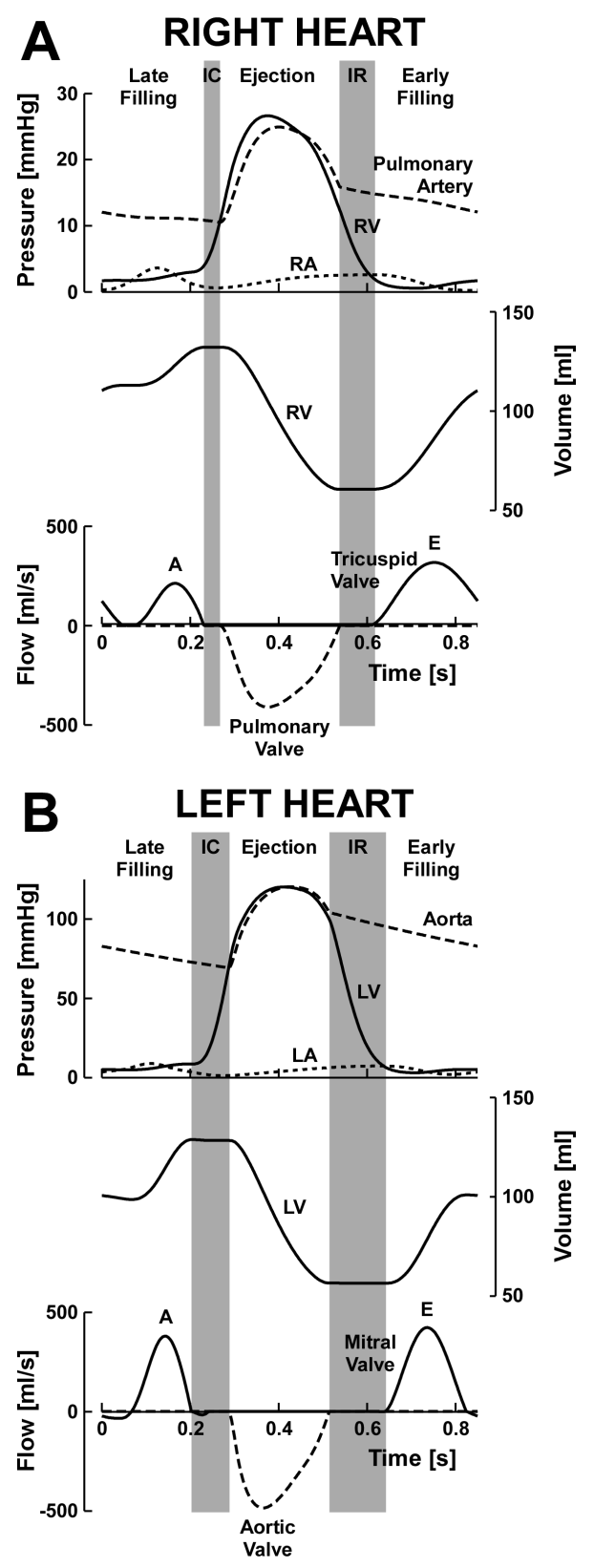

Figure 2.3 Time courses of blood pressures, ventricular volumes, and blood flows through cardiac valves during a cardiac cycle of a normal adult at rest. A: right heart hemodynamics. B: left heart hemodynamics. Note that the cardiac cycle is divided into four phases separated by moments of cardiac valve opening and closure, i.e., ventricular filling, isovolumic contraction (IC), ventricular ejection, and isovolumic relaxation (IR). Note that the filling phase is subdivided into an early passive filling wave (E) and a late active filling wave (A) induced by atrial contraction. Abbreviations: $L A=$ left atrial; $L V=$ left ventricular; $\mathrm{RA}=$ right atrial; $\mathrm{RV}=$ right ventricular. 


\section{Assessment of Global Ventricular Pump Load and Local Myofiber load}

During the cardiac cycle, a loop is formed by ventricular cavity pressure as function of cavity volume (Figure 2.4A). This pressure-volume loop represents the global mechanical pump load of the ventricle during a single cardiac cycle. The area of the pressure-volume loop quantifies external mechanical stroke work as generated by the ventricle. As depicted for the LV in Figure 2.4A, the ventricular pressure-volume loop is bounded by two curves quantifying the passive and active mechanical behavior of the ventricle as a chamber.
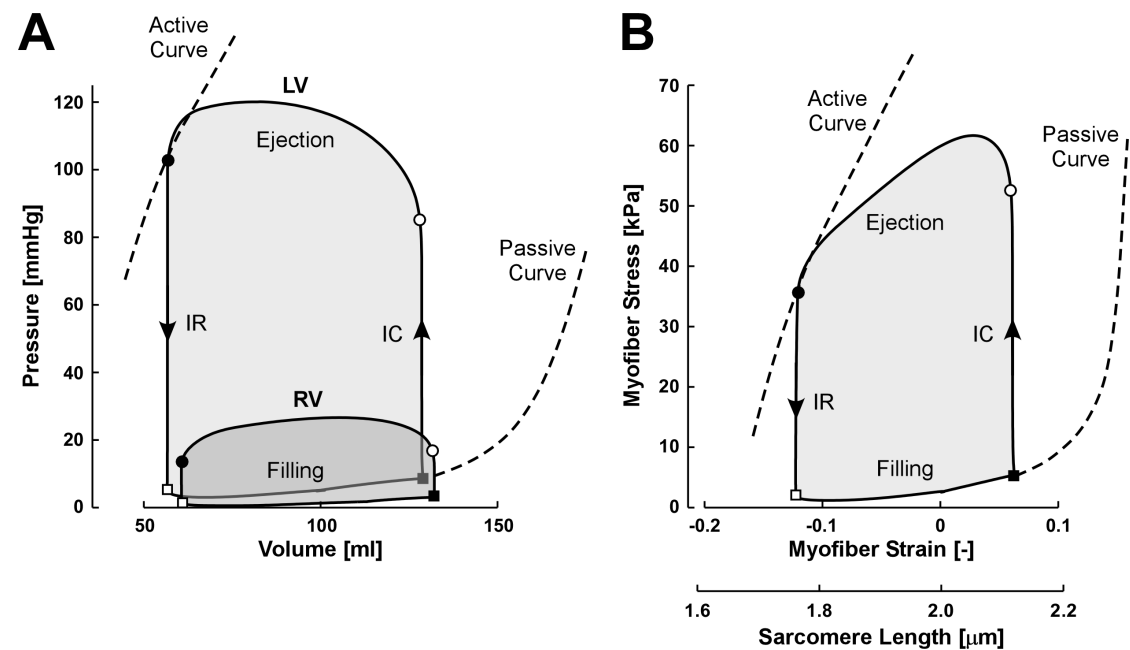

Figure 2.4 Assessment of ventricular mechanical load during a cardiac cycle of a normal adult at rest. A: global mechanical left (LV) and right (RV) ventricular pump load as expressed by cavity pressure-volume loops. The shaded area of the pressure-volume loop represents ventricular stroke work. B: local mechanical tissue load as expressed by a myofiber Cauchy stress-natural strain loop. The shaded area of the myofiber stress-strain loop represents myofiber stroke work density. Ventricular pressure-volume loops and myofiber stress-strain loops are bounded by two curves describing the passive and active behavior of the myocardium. Moments of aortic/pulmonary valve opening $(\circ)$ and closure $(\bullet)$ and of mitral/tricuspid valve opening $(\square)$ and closure $(\mathbf{})$ are indicated and divide the cardiac cycle in four phases, i.e., ventricular filling, isovolumic contraction (IC), ventricular ejection, and isovolumic relaxation (IR).

At local tissue level, a loop is formed by myofiber stress as function of myofiber strain during a cardiac cycle (Figure 2.4B). This myofiber stress-strain loop represents local mechanical load of the myofiber during a single cardiac 
cycle. In this thesis, myofiber stress is defined as Cauchy stress, i.e., the force per unit of cross-sectional area of the myofiber in the actual state. Myofiber strain is defined as natural strain, i.e., natural logarithm of the ratio of myofiber length to an arbitrary reference length. The area of the myofiber stress-strain loop quantifies mechanical stroke work density, i.e., contractile myofiber stroke work per unit of tissue volume. Analogous to the ventricular pressure-volume loop, the myofiber stress-strain loop is bounded by two curves quantifying the passive and active mechanical behavior of the local myocardial tissue (Figure 2.4B). Myofiber stress in passively stressed myocardial tissue increases about exponentially with myofiber strain. ${ }^{15,30}$ At low sarcomere length, myocardial tissue stiffness is mainly determined by components of the myocyte cytoskeleton such as titin. ${ }^{23,73}$ At larger sarcomere length, collagen in the extra-cellular matrix becomes stretched and, thereby, increases myocardial tissue stiffness and prevents myocardial overstretch during filling. Furthermore, passive mechanical behavior of the myocardial tissue is anisotropic, i.e., along the myofiber axis, the tissue is significantly stiffer than in the direction perpendicular to the myofiber axis. In the actively contracting state, myocardial tissue shows a more linear relation between myofiber stress and strain. Local force actively generated by the myofibers depends, among other parameters, on time after activation, sarcomere length, sarcomere shortening velocity, $12,26,36,64$ as well as on the mechanical boundary conditions imposed by the surrounding tissue.

Under normal cardiac loading conditions at rest, the RV ejects the blood against relatively low arterial pressure (mean pulmonary artery pressure $\approx 15$ $\mathrm{mmHg}$ ) as compared to the LV (mean aortic pressure $\approx 90 \mathrm{mmHg}$ ). Consequently, mechanical pump stroke work of the RV is about six times lower than that of the LV when assuming equal stroke volume. This difference in ventricular pump load is associated with differences in gross anatomy of both ventricles. The RV free wall is relatively thin compared to the LV free wall and the interventricular septum, the latter two having quite similar wall thicknesses (Figure 2.5, upper row). ${ }^{29}$ Furthermore, non-invasively obtained imaging data revealed that, in healthy subjects, the LV free wall accounts for about half of the total ventricular wall mass of about $200 \mathrm{~g}^{41}$ whereas the RV free wall and the interventricular septum both account for about a quarter.

The pump work generated by the LV and the RV is the resultant of the summed action of all individual myocytes in the ventricular walls. Myofiber stress, force, and cavity pressure are closely related quantities. Similarly, myofiber strain is closely related to changes in sarcomere length and cavity volume. The quantitative relation between global ventricular pump function, as expressed in 
terms of cavity pressure and volume, and local contractile tissue function, as expressed in terms of myofiber stress and strain, is not straightforward. Since it is hard to measure myofiber stress in vivo, ${ }^{19,44}$ researchers have used mathematical models to assess this relation. Most of these models are based on finite element analysis. $8,9,37,52,66,68$ Finite element models allow description of local wall mechanics and geometry and can, thus, deal with regional non-uniformities in the myocardial walls, e.g., asynchrony of activation or reduced contractile performance in case of myocardial infarction. Finite element models of the heart are computationally demanding due to their complexity. In an attempt to simplify the relation between myocardial tissue mechanics and pump function in the normal heart, Arts et al. ${ }^{1}$ found that the ratio of ventricular cavity to wall volume was the most important determinant for average myofiber stress and strain in the wall, while actual geometry appeared of minor importance. This finding was incorporated in the relatively simple one-fiber model relating ventricular cavity pressure and volume to myofiber stress and strain. The onefiber model was based on the assumption that, in the normal heart, myofiber structure and geometry adapt until myofiber load is evenly distributed over the myocardial wall. ${ }^{3}$ For study of beat-to-beat hemodynamics and mechanics of the heart and the blood vessels, the one-fiber concept has been used to develop the lumped closed-loop CircAdapt model of heart and circulation. ${ }^{2}$ The CircAdapt model consists of a network of modules representing myocardial walls, valves, large blood vessels, and peripheral resistances. It allows beat-to-beat simulation of time-dependent cardiovascular mechanics and hemodynamics, e.g., cavity volumes and pressures of the cardiac chambers, pressures in the large blood vessels, myofiber stress and strain in the cardiac walls, and flows through valves. An important feature of the CircAdapt model is that the number of independent parameters is reduced by incorporating adaptation of cardiac and vascular wall size and wall mass to mechanical load so that stresses and strains in the walls of heart and blood vessels are normalized to tissue-specific physiological standard levels. 2,43 


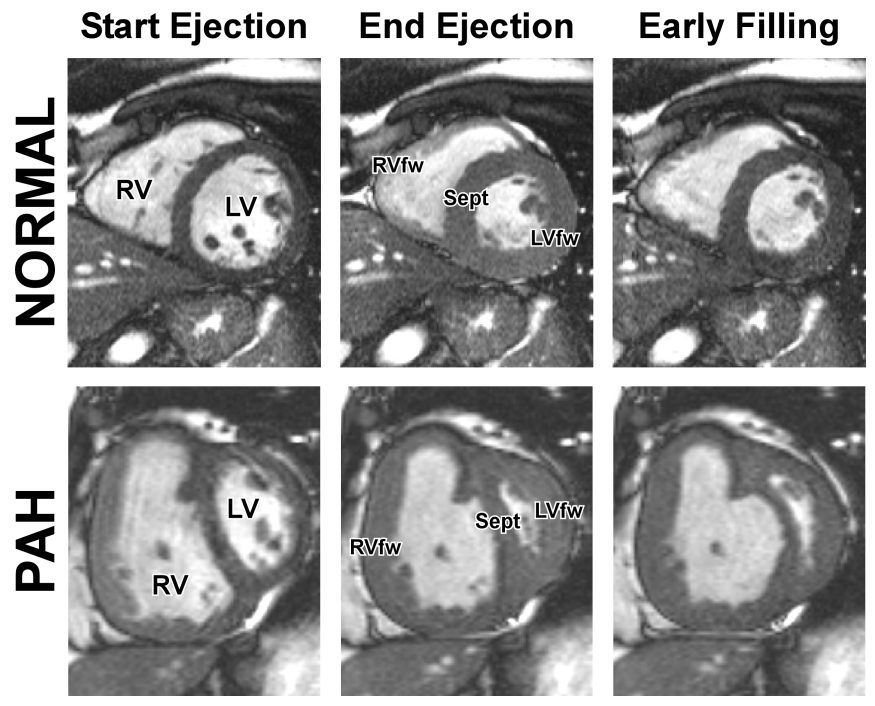

Figure 2.5 Midventricular short axis magnetic resonance images as obtained in a normal healthy adult (NORMAL) and in a patient with severe pulmonary arterial hypertension (PAH). In the normal heart, the septum (Sept) is concave toward the left ventricular (LV) cavity during the entire cardiac cycle. In the PAH heart, the Sept is concave toward the right ventricular (RV) cavity and shows most leftward bulging during early LV filling. Note the hypertrophic RV free wall (RVfw), the dilated RV cavity, and the small LV cavity in the PAH heart. Thickness of the LV free wall (LVfw) and of the septum are similar in the normal heart as well as in the PAH heart. The magnetic resonance images are used by courtesy of Marcus et al. ${ }^{47}$

\section{Ventricular Interaction}

Ventricular interaction refers to the concept of mutual mechanical and hemodynamic dependence of the LV and the RV. Three anatomic aspects of the cardiovascular system are responsible for ventricular interaction. First, hemodynamic interaction between the RV and the LV is achieved by their series coupling via the pulmonary circulation and the systemic circulation. In a normal heart under hemodynamically steady-state conditions, each ventricle ejects all the blood that it receives from the other ventricle. Second, the direct anatomical coupling of the ventricles results in a strong mechanical interaction via the interventricular septum. A change of shape, size, or pressure-volume relation of one ventricle affects shape, size, and pressure-volume relation of the other ventricle through the septum. 4, 16, 54, 58, 72 This phenomenon has been elegantly demonstrated with experiments in cardiac preparations in which direct mechanical ventricular interaction via the septum had been preserved but series 
ventricular interaction via the pulmonary circulation had been abolished. ${ }^{18,51,63,69}$ These studies showed that increase of RV pressure, while keeping LV pressure and filling volume artificially constant, increased LV end diastolic pressure as a direct result of geometrical changes such as leftward septal bulging. Third, the relatively stiff pericardial sac surrounding the heart limits acute cardiac distension and, thereby, modulates mechanical ventricular interaction. Experimental studies in animals and in patients have shown that pericardial constraint has little but significant effect on diastolic ventricular mechanics and negligible effect on systolic mechanics under normal physiological circumstances at rest. ${ }^{31,46}$ However, pericardial constraint has a significant effect on diastolic and systolic ventricular mechanics and hemodynamics with acute pulmonary hypertension or RV volume overload. ${ }^{5,6}$ Other studies showed that the pericardium is capable of adapting over time to changes in cardiac size and geometry. ${ }^{7,20}$

\section{Effect of Pulmonary Arterial Hypertension on Ventricular Pump Function and Anatomy}

With pulmonary arterial hypertension (PAH), progressive remodeling and vasoconstriction of the pulmonary vasculature leads to gradual increase of pulmonary vascular resistance. To maintain cardiac output, RV pressure is increased and may finally approach or even exceed LV pressure. In early stages of disease, the heart manages to compensate for the increase of $\mathrm{RV}$ afterload by increasing RV free wall mass (hypertrophy) so that wall stress is decreased to normal physiological value. ${ }^{11,25}$ In animal models of $\mathrm{PAH}^{50,53}$ the early hypertrophic response comprises significant increase of diameter and number of myocytes in the RV free wall, whereas myocyte length and average sarcomere length remained unchanged. With further aggravation of $\mathrm{PAH}$, however, RV contractile function deteriorates and RV end-diastolic volume increases. ${ }^{28,} 59$ Subsequently, wall tension and myocardial oxygen demand increase in the RV free wall, while coronary perfusion is locally diminished. ${ }^{67}$ Finally, these morphological and functional changes lead to decompensated right heart failure characterized by rise of RV filling pressures, development of RV diastolic dysfunction, RV dilatation, and decrease of cardiac output. ${ }^{70}$ Several RV diastolic function parameters correlate well with mean pulmonary artery pressure and with prognostic parameters assessing the risk of heart failure in patients with PAH. ${ }^{21,74}$

In severe PAH, RV dilatation is usually associated with functional tricuspid regurgitation due to dilatation of the tricuspid annulus followed by inability of 
the tricuspid leaflets to coapt properly. The resultant increase of RV volume overload accelerates the transition from compensation to decompensated right heart failure. Significant reduction of RV afterload by surgical treatment has been shown to diminish tricuspid regurgitation in patients with PAH. ${ }^{10,57,65}$

In $\mathrm{PAH}$, the most distinct characteristic is the abnormal geometry and motion of the interventricular septum. ${ }^{32,56,62}$ Several studies showed that septal geometry and motion are of diagnostic and predictive significance in patients with PAH. ${ }^{35,38}$ As mentioned previously, curvature of the septum is directly related to its transmural pressure gradient. ${ }^{13,16,39,40,55}$ As depicted in the upper row of Figure 2.5 , the septum of the normal heart is concave toward the LV cavity during the entire cardiac cycle. However, with increase of RV pressure due to PAH, the septum flattens and may even bulge toward the LV cavity (Figure 2.5, lower row). In severe PAH, the septum often experiences a rapid leftward motion during the LV isovolumic relaxation phase and during early LV filling. During this period, pressure in the RV shortly exceeds that in the LV due to delayed RV pressure decay. ${ }^{62}$ Furthermore, shortening of the RV free wall is prolonged compared to that of the LV free wall and the septum. ${ }^{47,71}$ Although the latter left-right delay of shortening correlates with an estimate of wall tension in the RV free wall, ${ }^{47}$ the precise reason for this interventricular mechanical and hemodynamic asynchrony is still unknown. In Chapter 5 and Chapter 7, a computer model is used to investigate the mechanism of interventricular mechanical and hemodynamic asynchrony in PAH. Furthermore, it is tested whether early pacing of the RV free wall may improve interventricular mechanical synchrony and, hence, cardiac pump function in patients with severe PAH (Chapter 5).

In patients with $\mathrm{PAH}$, chronic increase of pulmonary vascular resistance is associated with reduced LV end-diastolic volume and abnormal LV filling pattern, ${ }^{14,22,27,42,45,48,49,71}$ i.e., early diastolic peak of mitral blood flow velocity appears smaller than the peak of mitral flow velocity related to left atrial contraction. This phenomenon might be due to leftward septal bulging, which is believed to hinder passive filling by compressing the LV. Alternatively, the inability of the RV to maintain normal stroke volume is believed to reduce preload of the LV. Although Gurudevan et al. ${ }^{27}$ suggested that the latter mechanism is the dominant disturbing factor in patients with chronic thromboembolic pulmonary hypertension, it is still unclear to what extent both mechanisms contribute to the change of LV filling hemodynamics in these patients. In Chapter 6, the separate contributions of both mechanisms are quantified in patients with chronic thromboembolic pulmonary hypertension to obtain better understanding of the mechanism by which PAH affects LV filling. 
Chapter 2 


\section{Cardiac Anatomy and Physiology}

\section{REFERENCES}

1. Arts T, Bovendeerd PH, Prinzen FW, and Reneman RS. Relation between left ventricular cavity pressure and volume and systolic fiber stress and strain in the wall. Biophys J 59: 93-102, 1991.

2. Arts T, Delhaas T, Bovendeerd P, Verbeek X, and Prinzen FW. Adaptation to mechanical load determines shape and properties of heart and circulation: The CircAdapt model. Am J Physiol Heart Circ Physiol 288: H1943-1954, 2005.

3. Arts T, Prinzen FW, Snoeckx LH, Rijcken JM, and Reneman RS. Adaptation of cardiac structure by mechanical feedback in the environment of the cell: A model study. Biophys J 66: 953-961, 1994.

4. Baker AE, Dani R, Smith ER, Tyberg JV, and Belenkie I. Quantitative assessment of independent contributions of pericardium and septum to direct ventricular interaction. Am J Physiol 275: H476-483, 1998.

5. Belenkie I, Dani R, Smith ER, and Tyberg JV. The importance of pericardial constraint in experimental pulmonary embolism and volume loading. Am Heart J 123: 733-742, 1992.

6. Belenkie I, Sas R, Mitchell J, Smith ER, and Tyberg JV. Opening the pericardium during pulmonary artery constriction improves cardiac function. J Appl Physiol 96: 917-922, 2004.

7. Blanchard DG, and Dittrich HC. Pericardial adaptation in severe chronic pulmonary hypertension. An intraoperative transesophageal echocardiographic study. Circulation 85: 1414-1422, 1992.

8. Bovendeerd PH, Arts T, Delhaas T, Huyghe JM, van Campen DH, and Reneman RS. Regional wall mechanics in the ischemic left ventricle: Numerical modeling and dog experiments. Am J Physiol 270: H398-410, 1996.

9. Bovendeerd PH, Arts T, Huyghe JM, van Campen DH, and Reneman RS. Dependence of local left ventricular wall mechanics on myocardial fiber orientation: A model study. J Biomech 25: 1129-1140, 1992.

10. Braunwald NS, Ross J, Jr., and Morrow AG. Conservative management of tricuspid regurgitation in patients undergoing mitral valve replacement. Circulation 35: 16369, 1967.

11. Chin $\mathrm{KM}, \mathrm{Kim} \mathrm{NH}$, and Rubin LJ. The right ventricle in pulmonary hypertension. Coron Artery Dis 16: 13-18, 2005.

12. de Tombe PP, and ter Keurs HE. Force and velocity of sarcomere shortening in trabeculae from rat heart. Effects of temperature. Circ Res 66: 1239-1254, 1990.

13. Dellegrottaglie S, Sanz J, Poon M, Viles-Gonzalez JF, Sulica R, Goyenechea M, Macaluso F, Fuster $V$, and Rajagopalan S. Pulmonary hypertension: Accuracy of detection with left ventricular septal-to-free wall curvature ratio measured at cardiac MR. Radiology 243: 63-69, 2007. 


\section{Chapter 2}

14. Dittrich HC, Chow LC, and Nicod PH. Early improvement in left ventricular diastolic function after relief of chronic right ventricular pressure overload. Circulation 80: 823830, 1989.

15. Dokos S, Smaill BH, Young AA, and LeGrice IJ. Shear properties of passive ventricular myocardium. Am J Physiol Heart Circ Physiol 283: H2650-2659, 2002.

16. Dong SJ, Smith ER, and Tyberg JV. Changes in the radius of curvature of the ventricular septum at end diastole during pulmonary arterial and aortic constrictions in the dog. Circulation 86: 1280-1290, 1992.

17. Eghbali M, Czaja MJ, Zeydel M, Weiner FR, Zern MA, Seifter S, and Blumenfeld OO. Collagen chain mrnas in isolated heart cells from young and adult rats. J Mol Cell Cardiol 20: 267-276, 1988.

18. Elzinga G, van Grondelle R, Westerhof N, and van den Bos GC. Ventricular interference. Am J Physiol 226: 941-947, 1974.

19. Feigl EO, Simon GA, and Fry DL. Auxotonic and isometric cardiac force transducers. J Appl Physiol 23: 597-600, 1967.

20. Freeman GL, and LeWinter MM. Pericardial adaptations during chronic cardiac dilation in dogs. Circ Res 54: 294-300, 1984.

21. Gan CT, Holverda S, Marcus JT, Paulus WJ, Marques KM, Bronzwaer JG, Twisk JW, Boonstra A, Postmus PE, and Vonk-Noordegraaf A. Right ventricular diastolic dysfunction and the acute effects of sildenafil in pulmonary hypertension patients. Chest 132: 11-17, 2007.

22. Gan CT, Lankhaar JW, Marcus JT, Westerhof N, Marques KM, Bronzwaer JG, Boonstra A, Postmus PE, and Vonk-Noordegraaf A. Impaired left ventricular filling due to right-to-left ventricular interaction in patients with pulmonary arterial hypertension. Am J Physiol Heart Circ Physiol 290: H1528-1533, 2006.

23. Granzier HL, and Irving TC. Passive tension in cardiac muscle: Contribution of collagen, titin, microtubules, and intermediate filaments. Biophys J 68: 1027-1044, 1995.

24. Greenbaum RA, Ho SY, Gibson DG, Becker AE, and Anderson RH. Left ventricular fibre architecture in man. Br Heart J 45: 248-263, 1981.

25. Grossman W, Jones D, and McLaurin LP. Wall stress and patterns of hypertrophy in the human left ventricle. J Clin Invest 56: 56-64, 1975.

26. Guccione JM, Le Prell GS, de Tombe PP, and Hunter WC. Measurements of active myocardial tension under a wide range of physiological loading conditions. J Biomech 30: 189-192, 1997.

27. Gurudevan SV, Malouf PJ, Auger WR, Waltman TJ, Madani M, Raisinghani AB, DeMaria AN, and Blanchard DG. Abnormal left ventricular diastolic filling in chronic thromboembolic pulmonary hypertension: True diastolic dysfunction or left ventricular underfilling? J Am Coll Cardiol 49: 1334-1339, 2007. 


\section{Cardiac Anatomy and Physiology}

28. Hardziyenka M, Campian ME, de Bruin-Bon HA, Michel MC, and Tan HL. Sequence of echocardiographic changes during development of right ventricular failure in rat. J Am Soc Echocardiogr 19: 1272-1279, 2006.

29. Ho SY, and Nihoyannopoulos P. Anatomy, echocardiography, and normal right ventricular dimensions. Heart 92 Suppl 1: i2-13, 2006.

30. Hunter PJ, McCulloch AD, and ter Keurs HE. Modelling the mechanical properties of cardiac muscle. Prog Biophys Mol Biol 69: 289-331, 1998.

31. Janicki JS, and Weber KT. The pericardium and ventricular interaction, distensibility, and function. Am J Physiol 238: H494-503, 1980.

32. Jessup M, Sutton MS, Weber KT, and Janicki JS. The effect of chronic pulmonary hypertension on left ventricular size, function, and interventricular septal motion. Am Heart J 113: 1114-1122, 1987.

33. Jugdutt BI. Remodeling of the myocardium and potential targets in the collagen degradation and synthesis pathways. Curr Drug Targets Cardiovasc Haematol Disord 3: 1-30, 2003.

34. Jugdutt BI. Ventricular remodeling after infarction and the extracellular collagen matrix: When is enough enough? Circulation 108: 1395-1403, 2003.

35. Kaul S. The interventricular septum in health and disease. Am Heart J 112: 568-581, 1986.

36. Kentish JC, ter Keurs HE, Ricciardi L, Bucx JJ, and Noble MI. Comparison between the sarcomere length-force relations of intact and skinned trabeculae from rat right ventricle. Influence of calcium concentrations on these relations. Circ Res 58: 755-768, 1986.

37. Kerckhoffs RC, Neal ML, Gu Q, Bassingthwaighte JB, Omens JH, and McCulloch AD. Coupling of a $3 \mathrm{~d}$ finite element model of cardiac ventricular mechanics to lumped systems models of the systemic and pulmonic circulation. Ann Biomed Eng 35: 1-18, 2007.

38. King ME, Braun $H$, Goldblatt A, Liberthson R, and Weyman AE. Interventricular septal configuration as a predictor of right ventricular systolic hypertension in children: A crosssectional echocardiographic study. Circulation 68: 68-75, 1983.

39. Kingma I, Tyberg JV, and Smith ER. Effects of diastolic transseptal pressure gradient on ventricular septal position and motion. Circulation 68: 1304-1314, 1983.

40. Little WC, Reeves RC, Arciniegas J, Katholi RE, and Rogers EW. Mechanism of abnormal interventricular septal motion during delayed left ventricular activation. Circulation 65: 1486-1491, 1982.

41. Lorenz CH, Walker ES, Morgan VL, Klein SS, and Graham TP, Jr. Normal human right and left ventricular mass, systolic function, and gender differences by cine magnetic resonance imaging. J Cardiovasc Magn Reson 1: 7-21, 1999.

42. Louie EK, Rich S, and Brundage BH. Doppler echocardiographic assessment of impaired left ventricular filling in patients with right ventricular pressure overload due to primary pulmonary hypertension. J Am Coll Cardiol 8: 1298-1306, 1986. 


\section{Chapter 2}

43. Lumens J, Delhaas T, Kirn B, and Arts T. Three-wall segment (TriSeg) model describing mechanics and hemodynamics of ventricular interaction. Ann Biomed Eng 37: 22342255, 2009.

44. Lunkenheimer PP, Redmann K, Florek J, Fassnacht U, Cryer CW, Wubbeling F, Niederer P, and Anderson $\mathrm{RH}$. The forces generated within the musculature of the left ventricular wall. Heart 90: 200-207, 2004.

45. Mahmud E, Raisinghani A, Hassankhani A, Sadeghi HM, Strachan GM, Auger W, DeMaria AN, and Blanchard DG. Correlation of left ventricular diastolic filling characteristics with right ventricular overload and pulmonary artery pressure in chronic thromboembolic pulmonary hypertension. J Am Coll Cardiol 40: 318-324, 2002.

46. Mangano DT, Van Dyke DC, Hickey RF, and Ellis RJ. Significance of the pericardium in human subjects: Effects on left ventricular volume, pressure and ejection. J Am Coll Cardiol 6: 290-295, 1985.

47. Marcus JT, Gan CT, Zwanenburg JJ, Boonstra A, Allaart CP, Gotte MJ, and VonkNoordegraaf A. Interventricular mechanical asynchrony in pulmonary arterial hypertension: Left-to-right delay in peak shortening is related to right ventricular overload and left ventricular underfilling. J Am Coll Cardiol 51: 750-757, 2008.

48. Marcus JT, Vonk Noordegraaf A, Roeleveld RJ, Postmus PE, Heethaar RM, Van Rossum $A C$, and Boonstra A. Impaired left ventricular filling due to right ventricular pressure overload in primary pulmonary hypertension: Noninvasive monitoring using mri. Chest 119: 1761-1765, 2001

49. Menzel T, Wagner S, Kramm T, Mohr-Kahaly S, Mayer E, Braeuninger S, and Meyer J. Pathophysiology of impaired right and left ventricular function in chronic embolic pulmonary hypertension: Changes after pulmonary thromboendarterectomy. Chest 118 : 897-903, 2000.

50. Minami S, Onodera T, Okazaki F, Miyazaki H, Ohsawa S, and Mochizuki S. Myocyte morphological characteristics differ between the phases of pulmonary hypertensioninduced ventricular hypertrophy and failure. Int Heart J 47: 629-637, 2006.

51. Moulopoulos SD, Sarcas A, Stamatelopoulos S, and Arealis E. Left ventricular performance during by-pass or distension of the right ventricle. Circ Res 17: 484-491, 1965.

52. Nash MP, and Hunter PJ. Computational mechanics of the heart: From tissue structure to ventricular function. J Elasticity 61: 113-141, 2000.

53. Olivetti G, Ricci R, Lagrasta C, Maniga E, Sonnenblick EH, and Anversa P. Cellular basis of wall remodeling in long-term pressure overload-induced right ventricular hypertrophy in rats. Circ Res 63: 648-657, 1988.

54. Olsen CO, Tyson GS, Maier GW, Spratt JA, Davis JW, and Rankin JS. Dynamic ventricular interaction in the conscious dog. Circ Res 52: 85-104, 1983. 


\section{Cardiac Anatomy and Physiology}

55. Reisner SA, Azzam Z, Halmann M, Rinkevich D, Sideman S, Markiewicz W, and Beyar R. Septal/free wall curvature ratio: A noninvasive index of pulmonary arterial pressure. J Am Soc Echocardiogr 7: 27-35, 1994.

56. Roeleveld RJ, Marcus JT, Faes TJ, Gan TJ, Boonstra A, Postmus PE, and VonkNoordegraaf A. Interventricular septal configuration at mr imaging and pulmonary arterial pressure in pulmonary hypertension. Radiology 234: 710-717, 2005.

57. Sadeghi HM, Kimura BJ, Raisinghani A, Blanchard DG, Mahmud E, Fedullo PF, Jamieson SW, and DeMaria AN. Does lowering pulmonary arterial pressure eliminate severe functional tricuspid regurgitation? Insights from pulmonary thromboendarterectomy. J Am Coll Cardiol 44: 126-132, 2004.

58. Slinker BK, and Glantz SA. End-systolic and end-diastolic ventricular interaction. Am J Physiol 251: H1062-1075, 1986.

59. Spann JF, Jr., Covell JW, Eckberg DL, Sonnenblick EH, Ross J, Jr., and Braunwald E. Contractile performance of the hypertrophied and chronically failing cat ventricle. Am J Physiol 223: 1150-1157, 1972.

60. Spinale FG. Myocardial matrix remodeling and the matrix metalloproteinases: Influence on cardiac form and function. Physiol Rev 87: 1285-1342, 2007.

61. Streeter DD, Jr., Spotnitz HM, Patel DP, Ross J, Jr., and Sonnenblick EH. Fiber orientation in the canine left ventricle during diastole and systole. Circ Res 24: 339-347, 1969.

62. Tanaka H, Tei C, Nakao S, Tahara M, Sakurai S, Kashima T, and Kanehisa T. Diastolic bulging of the interventricular septum toward the left ventricle. An echocardiographic manifestation of negative interventricular pressure gradient between left and right ventricles during diastole. Circulation 62: 558-563, 1980.

63. Taylor RR, Covell JW, Sonnenblick EH, and Ross J, Jr. Dependence of ventricular distensibility on filling of the opposite ventricle. Am J Physiol 213: 711-718, 1967.

64. ter Keurs HE, Rijnsburger WH, van Heuningen R, and Nagelsmit MJ. Tension development and sarcomere length in rat cardiac trabeculae. Evidence of lengthdependent activation. Circ Res 46: 703-714, 1980.

65. Thistlethwaite PA, and Jamieson SW. Tricuspid valvular disease in the patient with chronic pulmonary thromboembolic disease. Curr Opin Cardiol 18: 111-116, 2003.

66. Usyk TP, Le Grice IJ, and McCulloch AD. Computational model of three-dimensional cardiac electromechanics. Comput Visual Sci 4: 249-257, 2002.

67. van Wolferen SA, Marcus JT, Westerhof $N$, Spreeuwenberg MD, Marques KM, Bronzwaer JG, Henkens IR, Gan CT, Boonstra A, Postmus PE, and Vonk-Noordegraaf A. Right coronary artery flow impairment in patients with pulmonary hypertension. Eur Heart J 29: 120-127, 2008.

68. Vetter FJ, and McCulloch AD. Three-dimensional stress and strain in passive rabbit left ventricle: A model study. Ann Biomed Eng 28: 781-792, 2000. 
69. Visner MS, Arentzen CE, Crumbley AJ, 3rd, Larson EV, O'Connor MJ, and Anderson RW. The effects of pressure-induced right ventricular hypertrophy on left ventricular diastolic properties and dynamic geometry in the conscious dog. Circulation 74: 410-419, 1986.

70. Voelkel NF, Quaife RA, Leinwand LA, Barst RJ, McGoon MD, Meldrum DR, Dupuis J, Long CS, Rubin LJ, Smart FW, Suzuki YJ, Gladwin M, Denholm EM, and Gail DB. Right ventricular function and failure: Report of a national heart, lung, and blood institute working group on cellular and molecular mechanisms of right heart failure. Circulation 114: 1883-1891, 2006.

71. Vonk-Noordegraaf A, Marcus JT, Gan CT, Boonstra A, and Postmus PE. Interventricular mechanical asynchrony due to right ventricular pressure overload in pulmonary hypertension plays an important role in impaired left ventricular filling. Chest 128: 6285630S, 2005.

72. Weber KT, Janicki JS, Shroff S, and Fishman AP. Contractile mechanics and interaction of the right and left ventricles. Am J Cardiol 47: 686-695, 1981.

73. Wu Y, Cazorla O, Labeit D, Labeit S, and Granzier H. Changes in titin and collagen underlie diastolic stiffness diversity of cardiac muscle. J Mol Cell Cardiol 32: 2151-2162, 2000.

74. Yu CM, Sanderson JE, Chan S, Yeung L, Hung YT, and Woo KS. Right ventricular diastolic dysfunction in heart failure. Circulation 93: 1509-1514, 1996. 



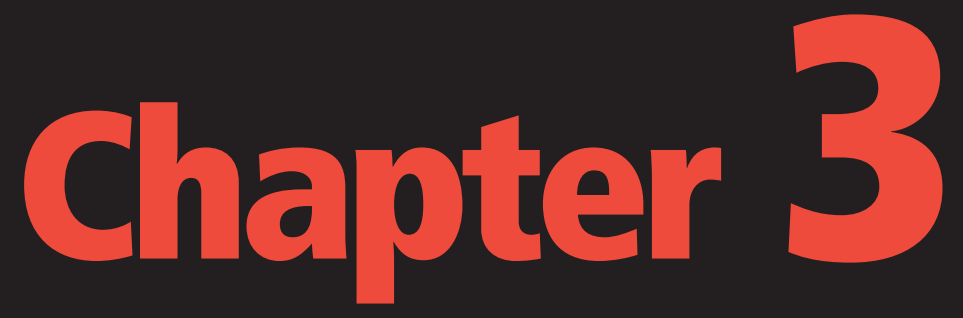

\title{
Modeling Ventricular Interaction:
}

a Multiscale Approach from Sarcomere Mechanics to Cardiovascular System Hemodynamics

\author{
Joost Lumens 1,2 \\ Tammo Delhaas \\ Borut Kirn 2,3 \\ Theo Arts ${ }^{2}$
}

Departments of ${ }^{1}$ Physiology and ${ }^{2}$ Biophysics, Cardiovascular Research Institute Maastricht (CARIM), Maastricht University, The Netherlands; ${ }^{3}$ Institute of Physiology, School of Medicine, University of Ljubljana, Slovenia.

Pac Symp Biocomput. 2008: 378-389, 2008 


\section{ABstract}

Direct ventricular interaction via the interventricular septum plays an important role in ventricular hemodynamics and mechanics. A large amount of experimental data demonstrates that left and right ventricular pump mechanics influence each other and that septal geometry and motion depend on transmural pressure. We present a lumped model of ventricular mechanics consisting of three wall segments that are coupled on the basis of balance laws stating mechanical equilibrium at the intersection of the three walls. The input consists of left and right ventricular volumes and an estimate of septal wall geometry. Wall segment geometry is expressed as area and curvature and is related to sarcomere extension. With constitutive equations of the sarcomere, myofiber stress is calculated. The force exerted by each wall segment on the intersection, as a result of wall tension, is derived from myofiber stress. Finally, septal geometry and ventricular pressures are solved by achieving balance of forces. We implemented this ventricular module in a lumped model of the closed-loop cardiovascular system (CircAdapt model). The resulting multiscale model enables dynamic simulation of myofiber mechanics, ventricular cavity mechanics, and cardiovascular system hemodynamics. The model was tested by performing simulations with synchronous and asynchronous mechanical activation of the wall segments. The simulated results of ventricular mechanics and hemodynamics were compared with experimental data obtained before and after acute induction of left bundle branch block in dogs. The changes in simulated ventricular mechanics and septal motion as a result of the introduction of mechanical asynchrony were very similar to those measured in the animal experiments. In conclusion, the module presented describes ventricular mechanics including direct ventricular interaction realistically and thereby extends the physiological application range of the CircAdapt model. 


\section{INTRODUCTION}

The left (LV) and right ventricle (RV) of the heart are pumping blood in the systemic and pulmonary circulation, respectively. Although both ventricular cavities are completely separated, there is a strong mechanical interaction between the ventricles, because they share the same septal wall, separating the cavities. A vast amount of evidence demonstrates that septal shape and motion depend on transseptal pressure. ${ }^{7,8}$ Also, a change in pressure or volume load of one ventricle influences pumping characteristics of the other ventricle., 12,15

Various mathematical models have been designed to describe the consequences of mechanical left-right coupling by the septum for ventricular geometry and hemodynamics. ${ }^{5,6,11,14,16,18}$ Commonly, interaction is assumed to be global and linear, using coupling coefficients for pressures, volumes or compliances. An exception was found in the model by Beyar et al., ${ }^{5}$ which was based on the balance of forces between free walls and septum. The latter model was primarily designed for diastolic interaction and was not suited to implement the dynamic mechanics of myocardial contraction.

The CircAdapt model ${ }^{3}$ has been developed to simulate cardiovascular dynamics and hemodynamics of the closed-loop circulation. The model is configured as a network, composed of four types of modules, i.e., cardiac chamber, blood vessel, valve and flow resistance. The number of required independent input parameters was reduced tremendously by incorporating adaptation of geometry, e.g., size of ventricular cavities and thickness of walls, to mechanical load so that stresses and strains in the walls were normalized to physiological standard levels. Ventricular interaction was modeled as an outer myocardial wall, encapsulating both ventricles, and an inner wall around the LV cavity accommodating the pressure difference between LV and RV. This description is reasonable, as long as LV pressure largely exceeds RV pressure. However, for high RV pressures, the description is not accurate anymore.

Because of the need to describe pathologic circumstances with high RV pressure, a new model of left to right ventricular interaction was designed. This model should be symmetric in design, allowing RV pressure to exceed LV pressure. Furthermore, the new model should satisfy the following requirements to fit in the CircAdapt framework. 1) For given LV and RV volumes as input, LV and RV pressures should be calculated as a result. 2) The model should incorporate dynamic myofiber mechanics, responsible for pump action. 3) The model should satisfy conservation of energy, i.e., the total amount of contractile work, as generated by the myofibers, should equal the total amount of hydraulic 
pump work, as delivered by the ventricles.

In the present study, a model setup was found, satisfying abovementioned requirements. The LV and RV cavities are formed between an LV free wall segment and a septal wall segment and between the septal wall segment and an RV free wall segment, respectively. The area of each wall segment depends on myofiber length in that wall. Pressures are generated by wall tension in the curved wall segments. Equilibria of mechanical forces are used to restrict degrees of freedom for geometry.

The model was tested by manipulating timing of mechanical activation of the various wall segments. Consequences of left bundle branch block (LBBB) have been simulated for septal motion and timing of LV and RV pressure development. Model results were compared with experimental results reported earlier. ${ }^{1,8-10,13,20}$

\section{Methods}

\section{Model design}

In the model, LV and RV cavities are enclosed by an LV and an RV free wall segment (LVfw and RVfw, respectively). The cavities are separated by a shared septal wall segment (Sept) (Figure 3.1). The wall segments are modeled as thickwalled spherical segments. The segments are assumed to be mechanically coupled at midwall. Midwall surface is defined to divide the wall in two spherical shells of equal wall volume. Midwall geometry of a wall segment depends on two variables, i.e., the midwall bulge height of the spherical segment $\left(x_{m}\right.$ sept $)$, and the radius of the midwall boundary circle $\left(y_{m}\right)$ (Figure 3.1). Midwall curvature, area, and volume of a wall segment can be expressed as a function of these two variables. Since all three wall segments share the same circle of intersection, four variables are needed to describe complete ventricular geometry, i.e., $x_{m, R v f w} x_{m, S e p t}, x_{m, L v f w}$ and $y_{m}$.

The core of the CircAdapt model is a set of first-order differential equations describing state-variables such as ventricular cavity volumes and flows through cardiac valves as a function of time. ${ }^{3}$ The CircAdapt model requires that RV and $\mathrm{LV}$ cavity pressures are expressed as function of the related cavity volumes. Since in the new model ventricular geometry is defined by four parameters, and only two volumes are known as input values, two remaining geometric parameters have to be solved. This is done by stating equilibrium of forces in the intersection of the wall segments. In Figure 3.2, the sequence of calculations within the ventricular module is shown graphically. 


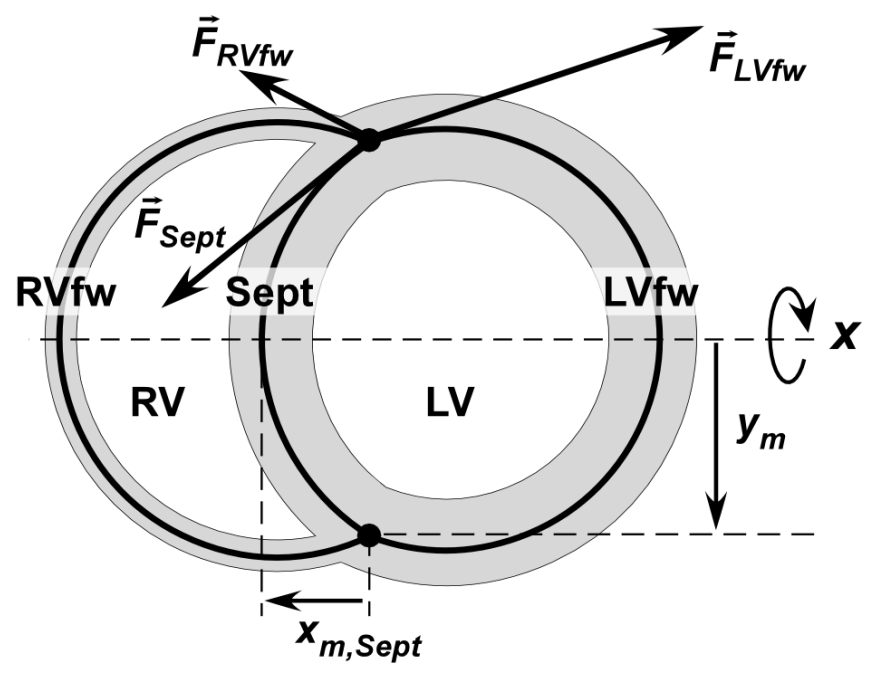

Figure 3.1: A cross-section of the model of ventricular mechanics. Three thick-walled spherical segments (shaded), i.e., the LV free wall segment (LVfw), the RV free wall segment (RVfw), and the septal wall segment (Sept) are coupled mechanically. The resulting ventricular composition is rotationally symmetric around axis $\mathrm{x}$ and has a midwall intersection circle crossing this image plane perpendicularly through the thick points. Midwall geometry of the septal wall segment is expressed by bulge height $\left(x_{m}\right.$, Sept $)$ and the radius $\left(y_{m}\right)$ of the midwall intersection circle. In this intersection each wall segment exerts a force $(F)$ caused by wall tension. 


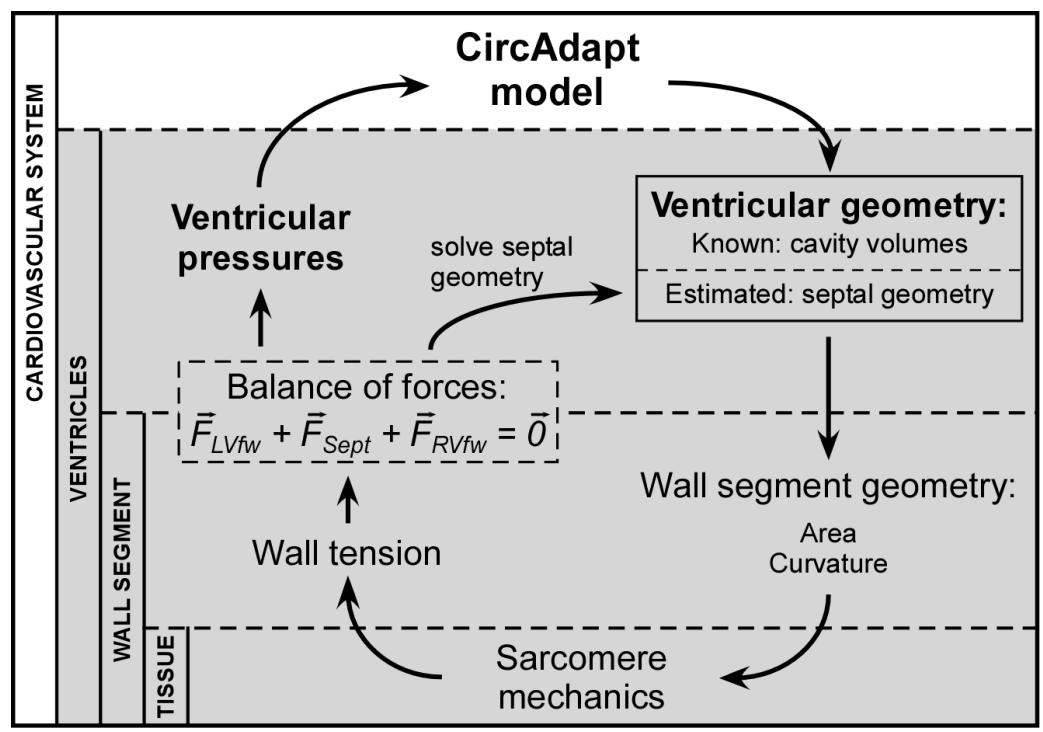

Figure 3.2: Flowchart of the new ventricular module (shaded area), describing ventricular mechanics up to and including the level of the myocardial tissue, as implemented within the framework of the CircAdapt model of the cardiovascular system. ${ }^{3}$ Ventricular pressures are calculated as a function of cavity volumes. Degrees of freedom in septal geometry are solved by achieving balance of forces. Then, ventricular cavity and wall mechanics as well as sarcomere mechanics are known.

Starting with LV and RV volumes and an estimate of septal bulge height $x_{m}$, sept and radius $y_{m}$ of the intersection circle, for all three segments, bulge height and segment radius are calculated. Next, for each segment, midwall area and curvature is calculated. From midwall area and curvature, sarcomere extension is calculated. Myofiber stress is calculated with constitutive equations of the sarcomere incorporating Hill's sarcomere force-velocity relation and Starling's sarcomere length-contractility relation, as previously described in detail by Arts et al. ${ }^{3}$ Using segment geometry, total radial and axial force components of midwall tension acting on the intersection circle are calculated. Thus, force balance provides two equations, which are solved numerically by proper variation of $x_{m, \text { Sept }}$ and $y_{m}$. Finally, a solution for ventricular geometry is found and LV and RV pressures are calculated from wall tensions, as needed for the CircAdapt model (Figure 3.2). 


\section{Simulation methods}

The model was tested by simulating canine ventricular hemodynamics and mechanics. The first simulation (Control) was assumed to be representative for baseline conditions with synchronously contracting ventricular wall segments. In a simulation of left bundle branch block (LBBB) we imposed asynchronous mechanical activation of the three wall segments, similar to that as observed in dogs with LBBB. ${ }^{19}$

Table 3.1 shows major input parameters used for the Control simulation, representing normal cardiac loading conditions of a dog. ${ }^{20,21}$ The thickness and midwall area of each wall segment were adapted to the loading conditions by using adaptation rules. ${ }^{3}$ The LBBB simulation represents an acute experiment in which no structural adaptation has occurred. Thus, with LBBB, size and weight of the wall segments were the same as in Control. Mechanical activation of the septum and LV free wall were delayed by $30 \mathrm{~ms}$ and $70 \mathrm{~ms}$ relative to the RV free wall, respectively. These average delay times were derived from animal experiments on mongrel dogs in which acute LBBB was induced by ablating the left branch of the His bundle using a radiofrequency catheter. ${ }^{20,21}$

Table 3.1. Input parameter values used for the simulations.

\begin{tabular}{lcc} 
Parameter & Unit & Value \\
\hline Mean arterial blood pressure & $\mathrm{kPa}$ & 10.8 \\
Cardiac output & $\mathrm{ml} / \mathrm{s}$ & 60 \\
Cardiac cycle time & $\mathrm{ms}$ & 760 \\
Blood pressure drop over & $\mathrm{kPa}$ & 1.33 \\
pulmonary circulation & & \\
\hline
\end{tabular}

The set of differential equations has been solved numerically using the ODE113 function in Matlab 7.1.0 (MathWorks, Natick, MA) with a temporal resolution of 2 ms. Simulation results were compared with experimental results of LV and RV pressure curves and the time course of septal motion. 


\section{RESULTS}

Simulation results of LV and RV hemodynamics for control and LBBB are shown in Figure 3.3. In the Control simulation, the time courses of pressures, volumes and flows are within the normal physiological range. In case of LBBB, the following hemodynamic changes, indicated by numbers in Figure 3.3, were noted:

1. LV pressure rise and decay were delayed with respect to that of RV pressure

2. Amplitude of the maximum positive time derivative of LV and RV pressureswere both decreased.

3. At the beginning of systole RV pressure exceeds LV pressure.

4. Beginning and end of LV ejection occur later than the corresponding RV events.

5. Mitral flow reverses after atrial contraction

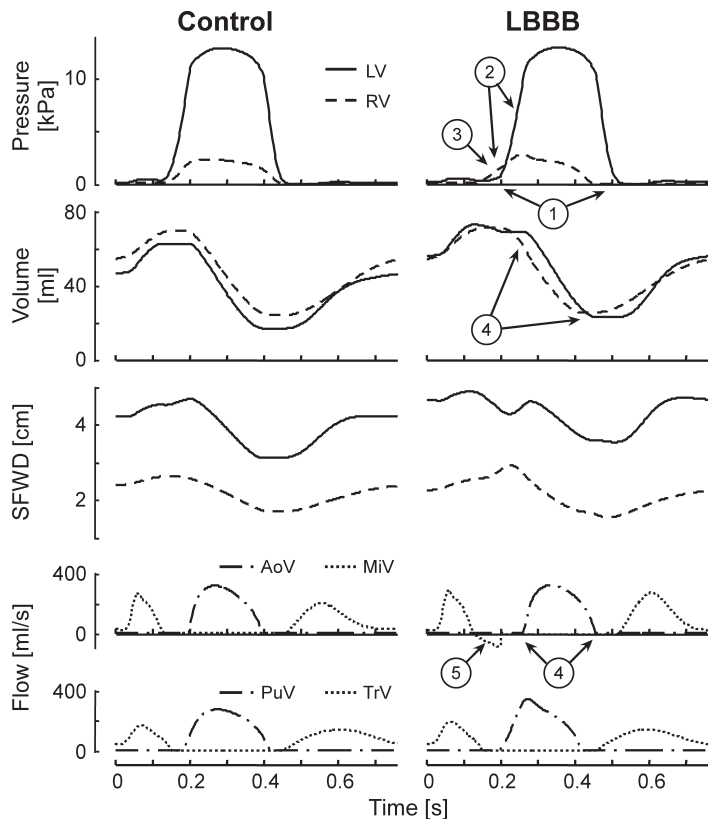

Figure 3.3: Time courses of left (LV) and right (RV) ventricular hemodynamics as simulated with the CircAdapt model in Control (left column) and with left bundle branch block (LBBB, right column). From top to bottom: LV and RV pressures, LV and RV volumes, septum-to-free wall distance (SFWD) for the LV and RV, flows through aortic (AoV) and mitral valves (MiV), and flows through pulmonary (PuV) and tricuspid valves (TrV). Encircled numbers correspond to changes listed in the text. 
In Figure 3.3, septal-to-free wall distances (SFWD) for both ventricles show also characteristic differences between Control and LBBB. In Control, time courses of RV and LV SFWD follow those of RV and LV volumes quite closely. With LBBB, the septum moves leftward during rise of RV pressure, and rightward shortly thereafter. During the rest of the cardiac cycle septal motion is similar in Control and LBBB.

Figure 3.4 shows LV and RV pressure curves normalized to their maximum value. The top panels show these normalized pressures, as obtained experimentally in a dog before and after induction of $\mathrm{LBBB} .{ }^{20}$ The bottom panels show the corresponding simulated curves. Experiment and simulation are in close agreement on the points already mentioned in relation to Figure 3.3. Moreover, in Figure 3.4, experiment and simulation appear in agreement on the increase of asymmetry of the RV pressure curve with LBBB.

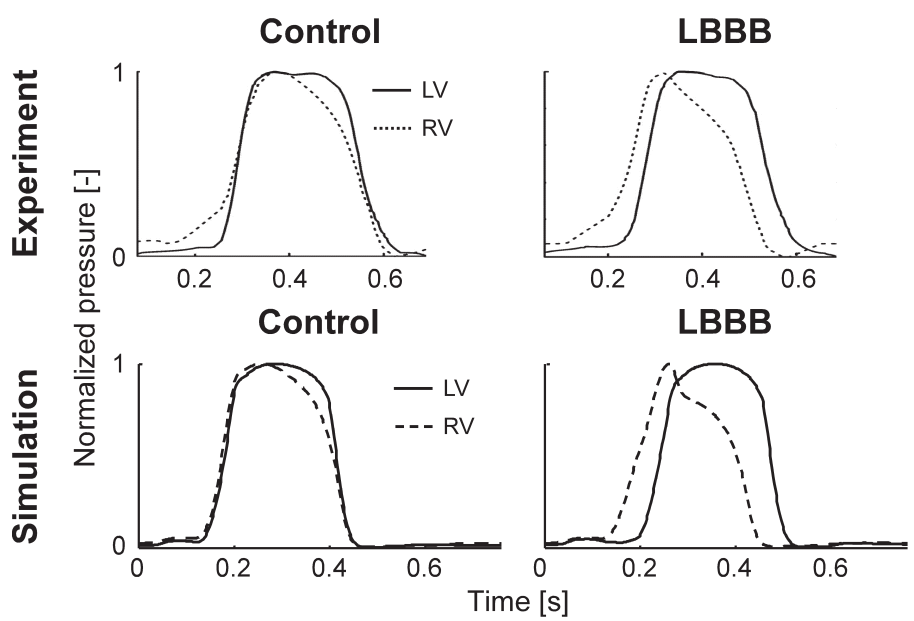

Figure 3.4: Left ventricular (LV) and right ventricular (RV) pressures normalized to their maxima. Top panels: representative experimental results of $L V$ and $R V$ pressures acquired before (Control) and after (LBBB) ablation of the left branch of the His-bundle in dogs. [Adapted from Verbeek et al. ${ }^{20}$ ] Bottom panels: normalized pressures obtained from the simulations shown in Figure 3.3.

Figure 3.5 shows LV SFWD as derived from typical M-Mode echocardiograms acquired in a dog before (Control) and after induction of LBBB. ${ }^{9}$ During LBBB, the experimental LV SFWD curve shows the same typical motion pattern of the septum early in systole as seen in the LBBB simulation. 

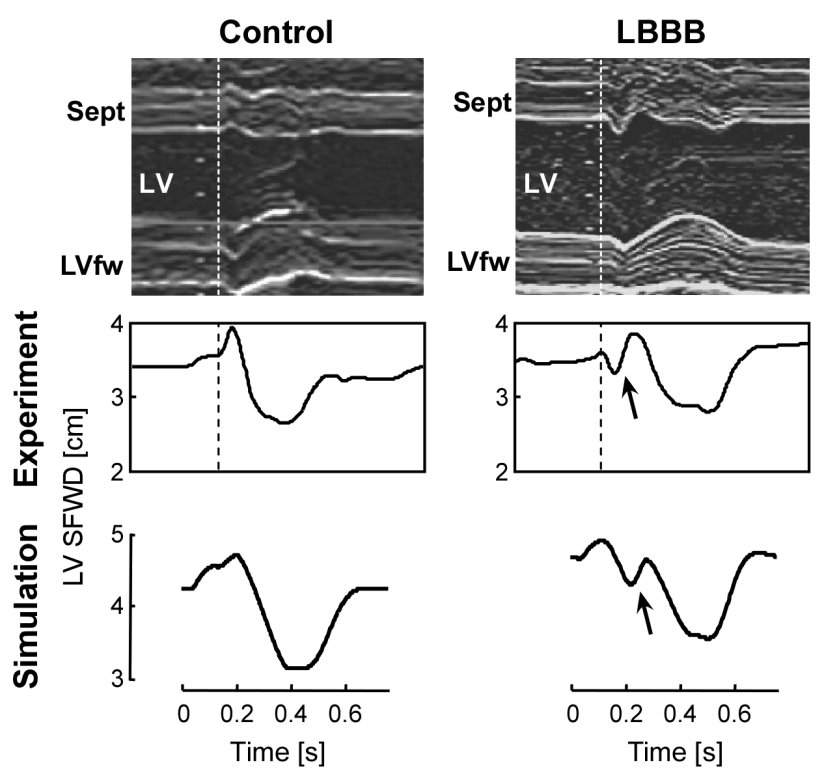

Figure 3.5: Left ventricular septal-to-free wall distance (LV SFWD) as derived from M-Mode echocardiograms of the left ventricle (LV) in the dog. [Adapted from Liu et al. ${ }^{9}$ ] The septal wall and LV free wall are indicated by Sept and LVfw, respectively. The left panel was acquired with synchronously contracting ventricles (Control) and the right image after induction of left bundle branch block (LBBB). Start of the QRS complex is indicated by vertical dashed lines. The arrows indicate the early systolic leftward motion of the septum, followed by the paradoxical rightward motion. The simulated curves of LV SFWD, as shown in the bottom panels, appear similar.

Figure 3.6 shows simulated LV and RV pressure-volume loops and myofiber stress-strain loops of all three wall segments. Stroke volumes do not change because cardiac output and heart rate were fixed in both simulations. In the LBBB simulation, the LV pressure-volume loop is shifted rightward, indicating ventricular dilatation that is generally considered representative for loss of cardiac contractile function. The areas of the stress-strain loops indicate contractile work of the myofibers per unit of tissue volume in the different wall segments. In Control circumstances, myocardial stroke work per unit of tissue volume is similar in all three segments, i.e., 5.5, 4.7, and $4.6 \mathrm{kPa}$ for LV free wall, septal wall, and RV free wall, respectively. With LBBB, the early activated RV free wall generates clearly less work per unit of tissue volume $(4.2 \mathrm{kPa})$ than the late activated LV free wall $(7.8 \mathrm{kPa})$. Although the septum is later activated than the $\mathrm{RV}$ free wall, the septal tissue generates far less work $(0.9 \mathrm{kPa})$. 

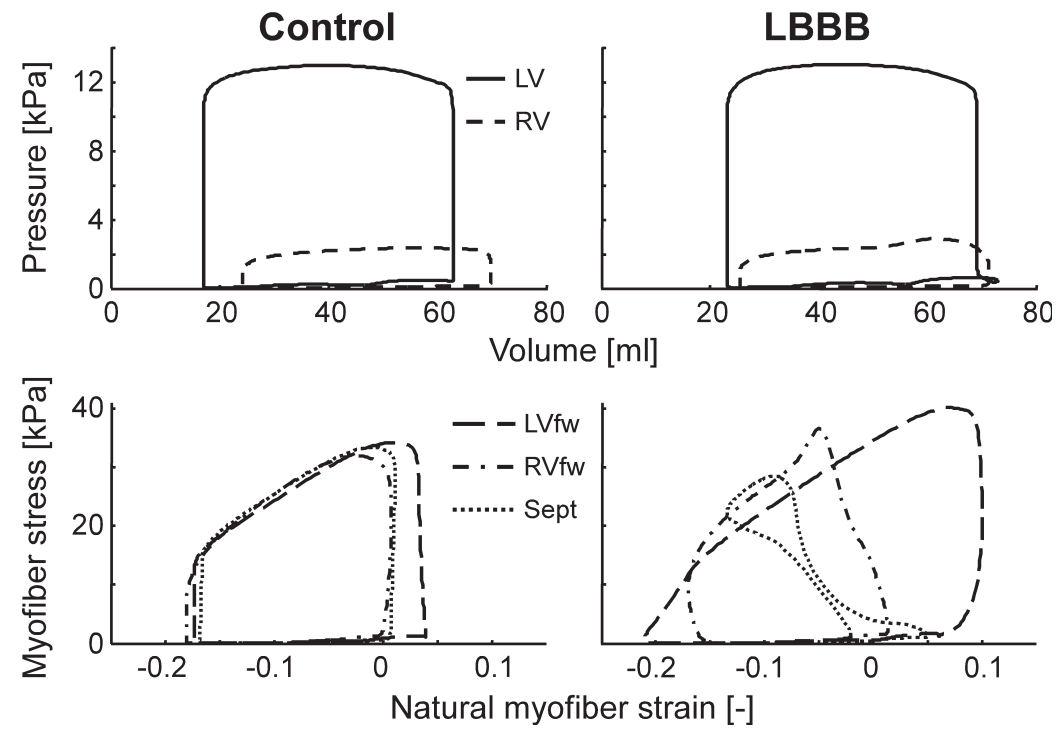

Figure 3.6: Simulated pressure-volume loops of the left ventricular (LV) and right ventricular (RV) cavities (top panels) and myofiber Cauchy stress-natural strain loops of the left ventricular free wall (LVfw), septal wall (Sept), and the right ventricular free wall (RVfw) (bottom panels). The left panels show results of the Control simulation and the right panels that of the LBBB simulation.

\section{Discussion}

A lumped module was designed, describing ventricular mechanics with direct ventricular interaction. The ventricular cavities were considered to be formed between three wall segments, being the LV free wall, septum and RV free wall. Mechanical interaction between the walls caused mutual dependency of LV and RV pump function. The three-segment ventricular module was incorporated in the closed-loop CircAdapt model of the complete circulation. Size and weight of the constituting wall segments were determined by adaptation of the myocardial tissue to imposed mechanical load. A comparison with experimental data ${ }^{9,13,20}$ demonstrated that simulation results of ventricular mechanics and hemodynamics at baseline and LBBB conditions were surprisingly realistic.

In the model, the atrioventricular valves could close only when the following two conditions were satisfied: 1) ventricular pressure exceeded atrial pressure and 2) the distal ventricular wall segments were mechanically activated. The latter condition mimicked papillary muscle function preventing valvular prolapse when ventricular pressure exceeded pressure in the proximal atrium. In 
the LBBB simulation, mitral backflow occurred because LV pressure rose above left atrial pressure before mechanical activation of the LV free wall. As soon as the LV free wall was activated, the mitral valve closed. Patient studies have shown that LBBB patients often have mitral regurgitation possibly as a result of late activation of papillary muscles. ${ }^{17}$

Figure 3.6 showed remarkable changes in the amount of myofiber work done by early and late activated wall segments of the LV. The same qualitative changes in LV regional myofiber work density have been observed in animal experiments in which regional LV pump work was derived from strain analysis of short-axis MR tagging images and simultaneous invasive pressure measurements. ${ }^{13}$ In chronic LBBB, these regional differences in work density may be responsible for asymmetric remodeling of the LV wall. ${ }^{21}$

A crucial step in the calculation procedure was the estimation of sarcomere extension. The one-fiber model by Arts et al., ${ }^{2}$ related sarcomere extension to the ratio of cavity volume to wall volume. This model has previously been shown to be applicable to an anisotropic thick-walled structure like a myocardial wall when assuming rotational symmetry and homogeneity of mechanical load in the wall. In our new model, the relation between midwall area and sarcomere extension was derived by applying the one-fiber model to a closed spherical cavity. The resulting relation was then extended to a partial segment of the sphere by considering a fraction of the wall, having the same curvature, wall tension, and transmural pressure difference. The one-fiber model has been shown to be rather insensitive to wall geometry. ${ }^{2}$ We expected the present relation between midwall area, curvature, and transmural pressure also to be quite insensitive to actual geometry. However, this fact has not been proven.

The simulation results demonstrated that ventricular interaction through the septum is one very important mechanism for the hemodynamic changes associated with abnormal mechanical activation of the ventricular wall segments. However, another important potential mechanism might be changes in contractility due to asynchronous contraction within each wall segment. Due to its lumped character, this model did not allow description of regional interactions within each wall segment but was limited to the description of its average sarcomere mechanics.

Experimental data show a decrease of cardiac output by approximately $30 \%$ after induction of LBBB. ${ }^{20,21}$ In our simulations, however, cardiac output was the same in the Control and LBBB simulations. In the model, cardiac output affects the forces in the intersection of the three wall segments proportionally, provided LV and RV stroke volumes are the same. Thus, a change of cardiac 
output as observed in the experiments will only affect the amplitude of septal wall motion (Figure 3.5) but not its characteristic course in time.

The mechanical coupling of the three spherical wall segments resulted in a circle of intersection with two degrees of freedom, namely, radial and axial displacement. This ventricular composition resulted in simple equations relating wall segment geometry to sarcomere behavior. Implementation of this ventricular module in the CircAdapt model resulted in a closed-loop system model that related fiber mechanics within the cardiac and vascular walls to hemodynamics realistically. Calculation time was limited to 6 seconds per cardiac cycle on a regular personal computer. Furthermore, the model behaved symmetrically around zero septal curvature, so that inversion of transseptal pressure and septal bulging could be handled. In conclusion, the resulting ventricular module satisfied all requirements mentioned in the introduction.

\section{ConcLusion}

In the lumped CircAdapt model of the complete circulation, a new module was incorporated, representing the heart with realistic left to right ventricular interaction. The ventricular part of the heart was designed as a composition of the LV free wall, the septum, and the RV free wall, encapsulating the LV and RV cavities. In a test simulation, ventricular hemodynamics and septal motion during normal synchronous activation was compared with these variables during left bundle branch block. Simulated time courses of ventricular pressures and septal motion were in close agreement with experimental findings. The newly developed three-segment module, describing ventricular mechanics with direct ventricular interaction, is a promising tool for realistic simulation of right heart function and septal motion under normal as well as pathologic circumstances, using the framework of the CircAdapt model.

\section{ACKNOWLEDGMENTS}

This research was financially supported by Actelion Pharmaceuticals Nederland B.V. (Woerden, The Netherlands). 
Chapter 3 


\section{REFERENCES}

1. Abbasi AS, Eber LM, MacAlpin RN, and Kattus AA. Paradoxical motion of interventricular septum in left bundle branch block. Circulation 49: 423-427, 1974.

2. Arts T, Bovendeerd PH, Prinzen FW, and Reneman RS. Relation between left ventricular cavity pressure and volume and systolic fiber stress and strain in the wall. Biophys J 59: 93-102, 1991.

3. Arts T, Delhaas T, Bovendeerd P, Verbeek X, and Prinzen FW. Adaptation to mechanical load determines shape and properties of heart and circulation: The CircAdapt model. Am J Physiol Heart Circ Physiol 288: H1943-1954, 2005.

4. Baker AE, Dani R, Smith ER, Tyberg JV, and Belenkie I. Quantitative assessment of independent contributions of pericardium and septum to direct ventricular interaction. Am J Physiol 275: H476-483, 1998.

5. Beyar R, Dong SJ, Smith ER, Belenkie I, and Tyberg JV. Ventricular interaction and septal deformation: A model compared with experimental data. Am J Physiol 265: H20442056, 1993.

6. Chung DC, Niranjan SC, Clark JW, Jr., Bidani A, Johnston WE, Zwischenberger JB, and Traber DL. A dynamic model of ventricular interaction and pericardial influence. Am J Physiol 272: H2942-2962, 1997.

7. Kingma I, Tyberg JV, and Smith ER. Effects of diastolic transseptal pressure gradient on ventricular septal position and motion. Circulation 68: 1304-1314, 1983.

8. Little WC, Reeves RC, Arciniegas J, Katholi RE, and Rogers EW. Mechanism of abnormal interventricular septal motion during delayed left ventricular activation. Circulation 65: 1486-1491, 1982.

9. Liu L, Tockman B, Girouard S, Pastore J, Walcott G, KenKnight B, and Spinelli J. Left ventricular resynchronization therapy in a canine model of left bundle branch block. Am J Physiol Heart Circ Physiol 282: H2238-2244, 2002.

10. McDonald IG. Echocardiographic demonstration of abnormal motion of the interventricular septum in left bundle branch block. Circulation 48: 272-280, 1973.

11. Olansen JB, Clark JW, Khoury D, Ghorbel F, and Bidani A. A closed-loop model of the canine cardiovascular system that includes ventricular interaction. Comput Biomed Res 33: 260-295, 2000.

12. Olsen CO, Tyson GS, Maier GW, Spratt JA, Davis JW, and Rankin JS. Dynamic ventricular interaction in the conscious dog. Circ Res 52: 85-104, 1983.

13. Prinzen FW, Hunter WC, Wyman BT, and McVeigh ER. Mapping of regional myocardial strain and work during ventricular pacing: Experimental study using magnetic resonance imaging tagging. J Am Coll Cardiol 33: 1735-1742, 1999. 


\section{Chapter 3}

14. Santamore WP, and Burkhoff D. Hemodynamic consequences of ventricular interaction as assessed by model analysis. Am J Physiol 260: H146-157, 1991.

15. Slinker BK, and Glantz SA. End-systolic and end-diastolic ventricular interaction. Am J Physiol 251: H1062-1075, 1986.

16. Smith BW, Chase JG, Shaw GM, and Nokes RI. Simulating transient ventricular interaction using a minimal cardiovascular system model. Physiol Meas 27: 165-179, 2006.

17. Soyama A, Kono T, Mishima T, Morita H, Ito T, Suwa M, and Kitaura Y. Intraventricular dyssynchrony may play a role in the development of mitral regurgitation in dilated cardiomyopathy. J Card Fail 11: 631-637, 2005.

18. Sun Y, Beshara M, Lucariello RJ, and Chiaramida SA. A comprehensive model for rightleft heart interaction under the influence of pericardium and baroreflex. Am J Physiol 272: H1499-1515, 1997.

19. Verbeek XA, Auricchio A, Yu Y, Ding J, Pochet T, Vernooy K, Kramer A, Spinelli J, and Prinzen FW. Tailoring cardiac resynchronization therapy using interventricular asynchrony. Validation of a simple model. Am J Physiol Heart Circ Physiol 290: H968977, 2006.

20. Verbeek XA, Vernooy K, Peschar M, Van Der Nagel T, Van Hunnik A, and Prinzen FW. Quantification of interventricular asynchrony during LBBB and ventricular pacing. Am J Physiol Heart Circ Physiol 283: H1370-1378, 2002.

21. Vernooy K, Verbeek XA, Peschar M, Crijns HJ, Arts T, Cornelussen RN, and Prinzen FW. Left bundle branch block induces ventricular remodelling and functional septal hypoperfusion. Eur Heart J 26: 91-98, 2005. 



\title{
Chapter 4
}

\section{Three-Wall Segment (TriSeg) Model} Describing Mechanics and Hemodynamics of Ventricular Interaction

\author{
Joost Lumens \\ Tammo Delhaas \\ Borut Kirn 2,3 \\ Theo Arts 2
}

Departments of ${ }^{1}$ Physiology and ${ }^{2}$ Biomedical Engineering, Cardiovascular Research Institute Maastricht (CARIM), Maastricht University, The Netherlands; ${ }^{3}$ Institute of Physiology, School of Medicine, University of Ljubljana, Slovenia. 


\section{ABstract}

A mathematical model (TriSeg model) of ventricular mechanics incorporating mechanical interaction of the left and right ventricular free walls and the interventricular septum is presented. Global left and right ventricular pump mechanics were related to representative myofiber mechanics in the three ventricular walls, satisfying the principle of conservation of energy. The walls were mechanically coupled satisfying tensile force equilibrium in the junction. Wall sizes and masses were rendered by adaptation to normalize mechanical myofiber load to physiological standard levels. The TriSeg model was implemented in the previously published lumped closed-loop CircAdapt model of heart and circulation. Simulation results of cardiac mechanics and hemodynamics during normal ventricular loading, acute pulmonary hypertension, and chronic pulmonary hypertension (including load adaptation) agreed with clinical data as obtained in healthy volunteers and pulmonary hypertension patients. In chronic pulmonary hypertension, the model predicted right ventricular free wall hypertrophy, increased systolic pulmonary flow acceleration, and increased right ventricular isovolumic contraction and relaxation times. Furthermore, septal curvature decreased linearly with its transmural pressure difference. In conclusion, the TriSeg model enables realistic simulation of ventricular mechanics including interaction between left and right ventricular pump mechanics, dynamics of septal geometry, and myofiber mechanics in the three ventricular walls. 


\section{GLOSSARY OF TERMS}

\section{General}

LV

RV

LVfW

Sept

RVfw
Left ventricle / ventricular

Right ventricle / ventricular

Left ventricular free wall

Septal wall

Right ventricular free wall

\section{Geometry-related parameters}

\begin{tabular}{|c|c|c|}
\hline$V_{L V}$ & $m^{3}$ & Left ventricular cavity volume \\
\hline$V_{R V}$ & $m^{3}$ & Right ventricular cavity volume \\
\hline$V_{w}$ & $m^{3}$ & Wall volume of ventricular wall segment \\
\hline$V_{m}$ & $\mathrm{~m}^{3}$ & $\begin{array}{l}\text { Volume of spherical cap, formed by midwall surface } \\
\text { of wall segment }\end{array}$ \\
\hline$A_{m}$ & $m^{2}$ & Midwall surface area of curved wall segment \\
\hline$C_{m}^{m}$ & $m^{-1}$ & Curvature of midwall surface (reciprocal of radius) \\
\hline$x_{m}$ & $\mathrm{~m}$ & Maximal axial distance from midwall surface to origin \\
\hline$y_{m}$ & $\mathrm{~m}$ & Radius of midwall junction circle \\
\hline$z$ & - & $\begin{array}{l}\text { Ratio of wall thickness to midwall radius of curvature } \\
\text { of curved wall segment }\end{array}$ \\
\hline$\alpha$ & - & Half the opening angle of spherical midwall surface \\
\hline$\varepsilon_{f}$ & - & Natural myofiber strain \\
\hline
\end{tabular}

\section{Force-related parameters}

\begin{tabular}{|c|c|c|}
\hline$p_{L V}$ & $\mathrm{~Pa}$ & Left ventricular cavity pressure \\
\hline$p_{R V}$ & $\mathrm{~Pa}$ & Right ventricular cavity pressure \\
\hline$p_{\text {Trans }}$ & $\mathrm{Pa}$ & $\begin{array}{l}\text { Transmural pressure difference across curved wall } \\
\text { segment }\end{array}$ \\
\hline$\sigma_{f}$ & $\mathrm{~Pa}$ & Cauchy myofiber stress \\
\hline$T_{m}$ & $\mathrm{Nm}^{-1}$ & Representative midwall tension \\
\hline$T_{x}$ & $\mathrm{Nm}^{-1}$ & Axial midwall tension component \\
\hline$T_{y}$ & $\mathrm{Nm}^{-1}$ & Radial midwall tension component \\
\hline
\end{tabular}


Chapter 4 


\section{INTRODUCTION}

The left (LV) and right (RV) ventricular cavities of the normal human heart are separated from the intrathoracic space by the left and right ventricular free walls, respectively (Figure 4.1A). The cavities are mutually separated by the septal wall. In order to simulate LV and RV mechanics and hemodynamics, the close anatomic coupling between these two ventricles must be considered. Experimental data demonstrate that a change in loading condition of either ventricle directly influences pump function of the other ventricle. ${ }^{5,21,50,55,68} \mathrm{It}$ is also well recognized that septal geometry and motion depend on transseptal pressure difference. ${ }^{36,37,51}$ This dependency is best illustrated by flattening of the septum with increase of RV pressure relative to LV pressure (Figure 4.1B). Septal geometry and motion appeared to be of diagnostic and predictive significance in patients with pulmonary hypertension. ${ }^{32,35}$

Several mathematical models of ventricular mechanics have been developed to quantify the effect of ventricular interaction on cardiac function. In several lumped models of ventricular hemodynamics, ${ }^{7}, 13,41,43,48,53,56,60,61$ ventricular interaction is described by empirically determined coupling coefficients, quantifying interventricular cross-talk of pressures and volumes. The model designed by Beyar et al. ${ }^{7}$ describes ventricular interaction in a more mechanistic way. In this model, LV and RV cavities are enclosed by three ventricular walls. For given ventricular pressures, the mechanical equilibrium of tensile forces in the junction of the walls is used as constraint to predict ventricular geometry. The model is restricted to description of passive mechanics of walls lacking contractile myofiber properties. More recently, 3D finite element models of the cardiac ventricles were used to simulate ventricular pump function and local tissue mechanics, ${ }^{34,47,66}$ inherently including ventricular interaction via the septum. In comparison with lumped models of global ventricular mechanics, finite element models allow description of regional wall mechanics and geometry. Consequently, these models are computationally demanding.

For study of beat-to-beat hemodynamics and mechanics of heart and blood vessels, Arts et al. previously developed the closed-loop CircAdapt model of heart and circulation. ${ }^{3}$ In this model, mechanical interaction of the LV and RV has been simulated by a common outer wall, having a transmural pressure equal to RV pressure, encapsulating an inner wall, which represents the left ventricle. ${ }^{1}$ The inner wall encapsulates the LV cavity only and has a transmural pressure equal to the difference between LV and RV pressure. Under normal ventricular loading conditions, this model enables realistic simulation of global LV and RV 
pump mechanics. ${ }^{1,3}$ However, this model setup presumes RV pressure to be substantially lower than LV pressure during the whole cardiac cycle. This condition is not satisfied with pulmonary hypertension or with left-to-right asynchrony of electrical activation. Furthermore, septal geometry can not be simulated. Thus, this model can not be used to interpret this measurable signal that contains important information about the difference between LV and RV pressure. ${ }^{35,51}$ Therefore, we designed the TriSeg model of ventricular mechanics that realistically incorporates ventricular interaction via the interventricular septum.

In the TriSeg model, three wall segments, i.e., the LV free wall (LVfw), septal wall (Sept), and RV free wall (RVfw), meet in a junction margin, thus encapsulating the LV and RV cavities (Figure 4.2A). LVfw and RVfw geometries are calculated starting from an initial estimate of Sept geometry. For each wall segment, myofiber strain is calculated from midwall surface area relative to a reference area. From myofiber strain, myofiber stress is determined using constitutive equations describing sarcomere mechanics. Substitution of myofiber stress in the known geometry of the walls results in representative midwall tension, acting on the junction line. Next, septal geometry is adjusted so that equilibrium of tension is achieved, i.e., summed tension at the junction of the three walls is equal to zero. As a result, geometries of the three wall segments and the two cavities are known, together with stresses, tensions, and pressures.

The Triseg model of ventricular mechanics is designed to be incorporated as a module in the existing CircAdapt model simulating mechanics and hemodynamics of the whole circulation. ${ }^{3}$ According to the principles of CircAdapt, size and mass of the wall segments are determined by adaptation so that mechanical load of the myofibers is normalized to physiological standard levels. ${ }^{3,4}$ The TriSeg model, as integrated in the CircAdapt model, has been tested by simulation of time-dependent LV and RV mechanics and hemodynamics under normal ventricular loading conditions as well as with acute and chronic pulmonary hypertension (PH). PH has been simulated to test whether the TriSeg model realistically relates septal geometry to transseptal pressure. Simulation results have been compared with previously published experimental data on ventricular hemodynamics 9,62 and on the relation between septal geometry and transmural pressure ${ }^{17,51}$ in healthy volunteers and in patients with chronic PH. 


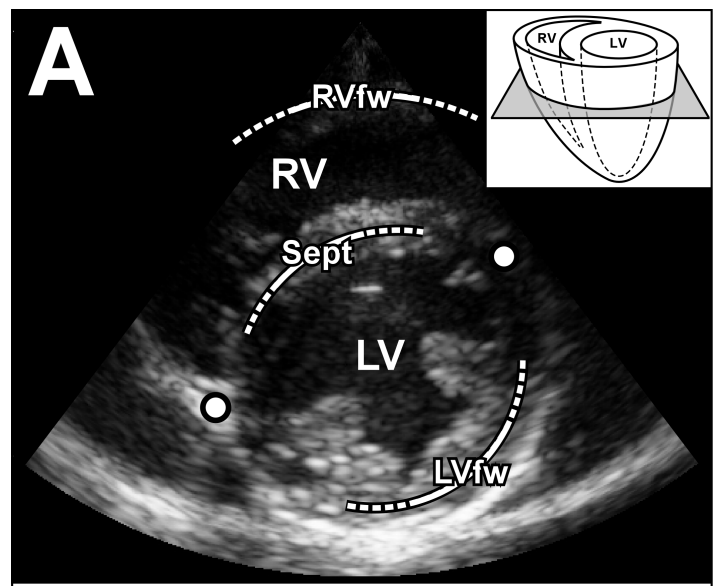

Figure 4.1: Two typical 2D echocardiographic images showing end-diastolic parasternal short-axis views of the hearts of a normal individual (A) and a pulmonary hypertension patient (B). Note that the septum is flattened with pulmonary hypertension. Crosssections of left (LV) and right (RV) ventricular cavities, LV free wall (LVfw), septal wall (Sept), and RV free wall (RVfw) are indicated. The bright dots indicate the cross-section of the junction margin of the walls. In the right upper corner, image plane orientation (shaded plane) is indicated in a schematic representation of the cardiac ventricles. 


\section{Methods}

\section{General design of the TriSeg model}

The design of the new TriSeg model of ventricular mechanics should enable incorporation as a module in the existing CircAdapt model that simulates mechanics and hemodynamics of the whole circulation. ${ }^{3}$ Therefore, the TriSeg model calculates LV and RV pressures ( $p_{L V}$ and $p_{R V}$, respectively) as functions of LV and RV cavity volumes ( $V_{L V}$ and $V_{R V}$, respectively). Mechanics of ventricular interaction is incorporated assuming a simplified ventricular composite geometry (Figure 4.2A). The three ventricular walls LVfw, Sept, and RVfw are modeled to be thick-walled and spherical with a common junction circle with midwall radius $y_{m}$ (Figure 4.2B). Midwall is defined as the spherical surface that divides wall volume $V_{w}$ in an inner and an outer shell of equal volume. Midwall volume $V_{m}$ is the volume enclosed by the midwall surface and the plane of the junction circle (Figure 4.2C). The center of the junction circle is the origin of the applied cylindrical coordinate system (Figure 4.2B). The $x$-direction is perpendicular to the plane of the junction circle and is defined positive towards the RV free wall. The $y$ coordinate represents the radial distance to the $x$-axis. The spherical midwall surface of a wall segment intersects the $x$-axis at value $x_{m}$ (Figure 4.2C). 

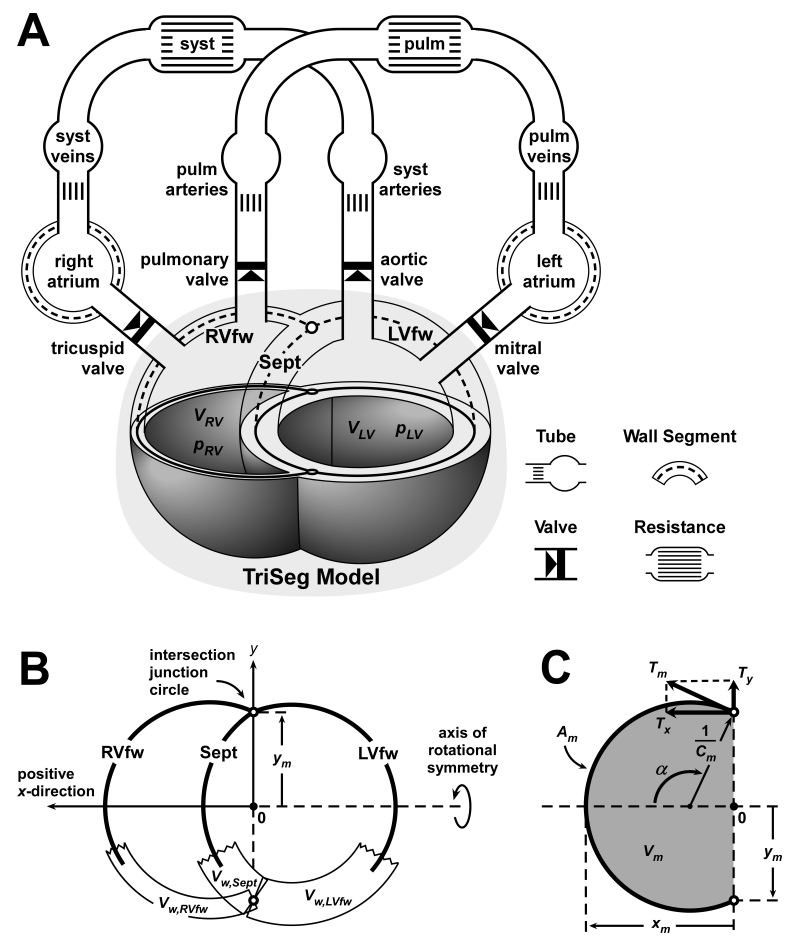

Figure 4.2: TriSeg model of ventricular mechanics. A: The TriSeg model (grey shading) incorporated in the modular CircAdapt model of the systemic (syst) and pulmonary (pulm) circulations. ${ }^{3}$ Three thick-walled spherical segments representing LV free wall (LVfw), RV free wall (RVfw), and septal wall (Sept) form a ventricular composite consisting of a left ventricular (LV) cavity, with volume $V_{L V}$ and pressure $p_{L V}$, and a right ventricular (RV) cavity, with volume $V_{R V}$ and pressure $p_{R V}$. B: Crosssection of the ventricular composite through the axis of rotational symmetry $(x)$. The ventricular walls are coupled mechanically at the common junction circle where their midwall surfaces (thick lines) meet. This junction circle perpendicularly intersects the plane of drawing (open dots). The midwall surface divides the wall with wall volume $V_{w}$ in two shells of equal volume. The center of the junction circle is the origin (0) of the cylindrical coordinate system applied. Note that $x$-direction is defined positive towards the RV free wall. C: Cross-section of a single wall segment through the axis of rotational symmetry. Note that only midwall geometry is shown. The shaded area indicates midwall volume $V_{m}$ enclosed between the midwall surface and the junction plane. Midwall volume $V_{m}$, area $A_{m}$, and curvature $C_{m}$ (reciprocal of radius of curvature) depend on distance $x_{m}$ and radius $y_{m}$ of the boundary circle. Each wall is loaded by transmural pressure difference resulting in a representative midwall tension $T_{m}$. With half opening angle $\alpha_{1} T_{m}$ is resolved in an axial $\left(T_{x}\right)$ and a radial $\left(T_{y}\right)$ component. 
In presenting the TriSeg model, the following levels are distinguished, 1) ventricular hemodynamics, 2) ventricular composite mechanics, 3) ventricular wall segment mechanics, 4) curved wall patch mechanics, and 5) myofiber mechanics. Starting from LV and RV volumes, the sequence of calculations needed to obtain LV and RV pressures has been illustrated in Figure 4.3. The ventricular hemodynamics section serves as interface with the CircAdapt model of the whole circulation. ${ }^{3}$ In the ventricular composite section, LV and RV volumes are used to calculate LV and RV pressures, applying a model of wall mechanics to each ventricular wall segment. In the ventricular wall segment section, volume $V_{m}$ and boundary radius $y_{m}$ of a spherical wall segment are used to calculate the axial and radial vector components $T_{x}$ and $T_{y}$, respectively, at the common junction circle from representative midwall tension $T_{m}$, applying a model of the mechanics of a curved wall patch. Such patch is defined as a fraction of a spherical wall segment with an arbitrarily shaped boundary and with midwall area $A_{m}$ and curvature $C_{m}$. Curvature is defined as the reciprocal of radius of curvature, having the advantage of being well-defined for a flat wall. In the section of curved wall patch mechanics, $A_{m}$ and $C_{m}$ are used to calculate representative midwall tension $T_{m}$, applying a model of myofiber mechanics. In the myofiber mechanics section, natural myofiber strain $\varepsilon_{f}$ is converted to Cauchy myofiber stress $\sigma_{f,}$ using a constitutive law of the myofiber. This model of myofiber mechanics has been heuristically obtained from reported physiological experiments. 


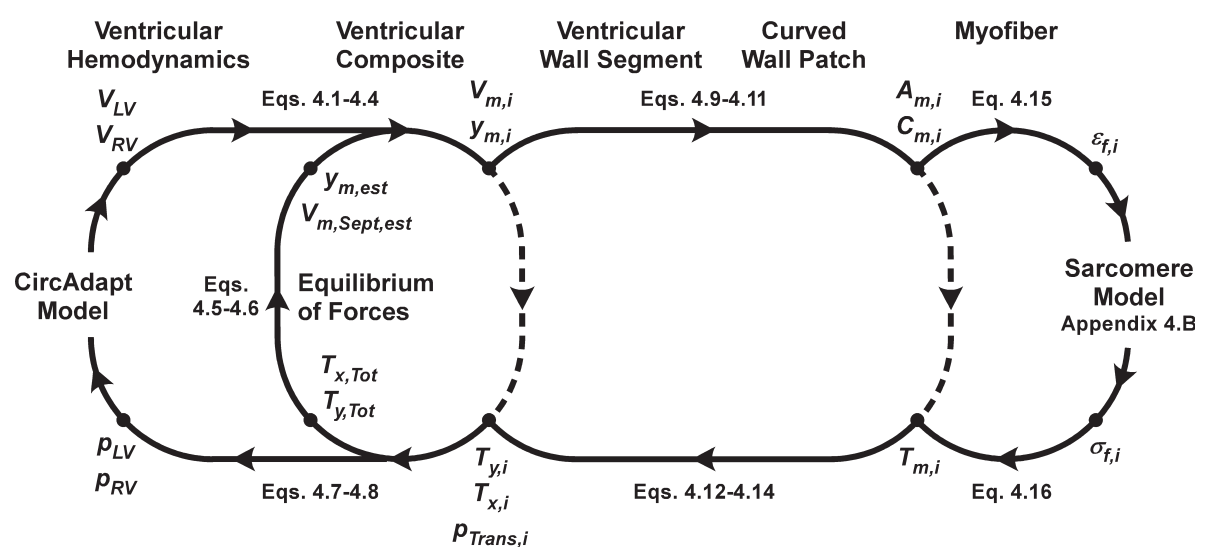

Figure 4.3: Flowchart of the TriSeg model of left (LV) and right (RV) ventricular mechanics. Applied equation numbers are indicated. The ventricular hemodynamics section serves as interface with the CircAdapt model of the whole circulation. In the ventricular composite section, for each wall $i$, midwall boundary radius $\left(V_{m, i}\right)$ and midwall volume $\left(V_{m, i}\right)$ are calculated from LV and RV volumes $\left(V_{L V}\right.$ and $V_{R V}$, respectively) and septal geometry. Septal geometry is initially defined by estimates of boundary radius $\left(y_{m, \text { est }}\right)$ and midwall volume $\left(V_{m, \text { Sept,est }}\right)$. LV free wall, septum, and RV free wall are indicated by $i=\mathrm{LVfw}$, Sept, and RVfw, respectively. The first dashed arrow indicates calculation of axial $\left(T_{x, i}\right)$ and radial $\left(T_{y, i}\right)$ wall tension components and transmural pressure $\left(p_{\text {Trans,i, }}\right)$. LV and RV pressure ( $p_{L V}$ and $p_{R V}$, respectively) and a new estimate of septal geometry is obtained from the summed axial $\left(T_{x, \text { Tot }}\right)$ and radial $\left(T_{y, T o t}\right)$ tension components. If septal geometry is sufficiently accurate by iteration, the solution is accepted. Because direct calculation along the dashed arrow is not possible, the sequence of calculations has to follow the solid lines. For each wall segment, midwall area $\left(A_{m, i}\right)$ and curvature $\left(C_{m, i}\right)$ are calculated. Wall tension $\left(T_{m, i}\right)$ is calculated following the second dashed arrow and renders wall tension components and transmural pressure. Again, direct calculation is not possible. Therefore, in the myofiber section, midwall area and curvature are used to calculate myofiber strain $\left(\varepsilon_{f, i}\right)$. Myofiber strain is converted to myofiber stress $\left(\sigma_{f, i}\right)$ using a model of sarcomere mechanics. Myofiber stress and wall segment geometry are used to calculate wall tension. By cascading the different sections, calculations can be performed sequentially along the pathways marked by solid lines. 


\section{Ventricular composite mechanics}

In this section, it is shown how cavity volumes $V_{L V}$ and $V_{R V}$ are used to calculate pressures $p_{L V}$ and $p_{R V}$. For that purpose, a model of ventricular wall segment mechanics is applied that renders axial and radial tension components $\left(T_{x}\right.$ and $T_{y}$, respectively) from representative midwall tension as a function of the midwall volume $\left(V_{m}\right)$ and junction radius $\left(y_{m}\right)$ of the spherical wall segment. This latter model will be derived in the next section discussing mechanics of the ventricular wall segment.

The geometry of the ventricular composite (Figure $4.2 B$ and $C$ ) is defined by the common radius $y_{m}$ of the wall junction and the enclosed midwall cap volumes $V_{m, L V f w}, V_{m \text {,Sept }}$ and $V_{m, R V f w}$ of the wall segments LVfW, Sept, and RVfW, respectively. Septal midwall volume $V_{m, \text { sept }}$ and junction radius $y_{m}$ are initially estimated by the solutions as obtained in the preceding time point, i.e., $V_{m \text {, Sept,est }}$ and $y_{m, e s t}$, respectively:

$$
\begin{aligned}
& y_{m}=y_{m, \text { est }} \\
& V_{m, \text { Sept }}=V_{m, \text { Sept,est }}
\end{aligned}
$$

The latter estimates, together with ventricular cavity volumes $V_{L V}$ and $V_{R V}$ as given directly by the CircAdapt model, render an estimate of ventricular composite geometry. Using the fact that ventricular cavity volume added to half of wall volume $V_{w}$ of the enclosing wall segments renders the sum of midwall volumes, it is found:

$$
\begin{aligned}
& V_{m, L V t w}=-V_{L V}-\frac{1}{2} V_{w, L V t w}-\frac{1}{2} V_{w, \text { Sept }}+V_{m, \text { Sept }} \\
& V_{m, \text { RVfw }}=+V_{R V}+\frac{1}{2} V_{W, R V \text { fiv }}+\frac{1}{2} V_{w, \text { Sept }}+V_{m, \text { Sept }}
\end{aligned}
$$

Note that the sign of midwall volume $V_{m}$ is positive if wall curvature is convex to the positive $x$-direction (Figure 4.2B). Thus, for a normal heart, the sign is negative for LVfw and positive for RVfw and Sept.

For each wall segment $i, V_{m, i}$ and $y_{m, i}$ are used to calculate axial and radial tension components $T_{x, i}$ and $T_{y, i}$ as well as transmural pressure $p_{\text {Trans,i. }}$. This is done by applying a model of ventricular wall segment mechanics as discussed in 
the next section. The axial tension components of the three wall segments $T_{x, L V f w} T_{x, \text { Sept }}$ and $T_{x, R V f w}$ are summed to calculate the net axial tension $T_{x, \text { Tot }}$ in the junction. Similarly, the net radial tension $T_{y, \text { tot }}$ in the junction is obtained. The initial estimates of $y_{m}$ and $V_{m \text {, sept }}$ (Eqs. 4.1 and 4.2) are adjusted numerically so that for the summed tension components it holds:

$$
\begin{aligned}
& T_{x, \text { Tot }}\left(y_{m}, V_{m, \text { Sept }}\right)=0 \\
& T_{y, \text { Tot }}\left(y_{m}, V_{m, \text { Sept }}\right)=0
\end{aligned}
$$

Having solved Eqs. 4.5 and 4.6, and assuming the pressure surrounding the ventricular composite to be zero, for LV and RV pressures it holds:

$$
\begin{aligned}
& p_{L V}=-p_{\text {Trans, LVfV }} \\
& p_{R V}=+p_{\text {Trans, RVfw }}
\end{aligned}
$$

Note that the minus sign for $p_{L V}$ is caused by the definition of $p_{\text {Trans, }}$ which is positive for a negative $x$-gradient of pressure in the wall segment.

\section{Mechanics of the ventricular wall segment}

In this section, it is shown for a wall segment (Figure 4.2C) how $V_{m}$ and $y_{m}$ are used to calculate axial and radial midwall tension components $T_{x}$ and $T_{y}$, and transmural pressure $p_{\text {Trans }}$. For that purpose, a model of the mechanics of a curved wall patch is applied. Such curved wall patch is defined as a fraction of a spherical wall segment with midwall surface area $A_{m}$ and curvature $C_{m}$. This latter model renders representative midwall tension from midwall surface area $A_{m}$ and curvature $C_{m}$ and will be derived in the next section discussing mechanics of the curved wall patch.

Wall segment geometry is most conveniently described by radius $y_{m}$ of the junction circle and distance $\mathrm{xm}$ between plane of junction circle and center of midwall surface (Figure 4.2C). $V_{m}, A_{m}$, and $C_{m}$ depend on $x_{m}$ and $y_{m}$ by

$$
\begin{aligned}
& V_{m}=\frac{\pi}{6} x_{m}\left(x_{m}^{2}+3 y_{m}^{2}\right) \\
& A_{m}=\pi\left(x_{m}^{2}+y_{m}^{2}\right)
\end{aligned}
$$




$$
C_{m}=\frac{2 x_{m}}{\left(x_{m}^{2}+y_{m}^{2}\right)}
$$

First, $x_{m}$ is calculated from $y_{m}$ and $V_{m}$ by solving Eq. 4.9. Second, $A_{m}$ and $C_{m}$ are determined with Eqs. 4.10 and 4.11. Next, $A_{m}$ and $C_{m}$ are used to calculate representative midwall tension $T_{m}$, applying a model of the mechanics of a curved wall patch as discussed in the next section.

At the circular boundary of the midwall surface (Figure 4.2C), geometric parameters $x_{m}$ and $y_{m}$, and midwall tension $T_{m}$ are used to calculate axial $\left(T_{x}\right)$ and radial $\left(T_{y}\right)$ tension components:

$$
\begin{aligned}
& T_{x}=T_{m} \cdot \sin \alpha \quad \text { with } \quad \sin \alpha=\frac{2 x_{m} y_{m}}{x_{m}^{2}+y_{m}^{2}} \\
& T_{y}=T_{m} \cdot \cos \alpha \quad \text { with } \quad \cos \alpha=\frac{-x_{m}^{2}+y_{m}^{2}}{x_{m}^{2}+y_{m}^{2}}
\end{aligned}
$$

The symbol $\alpha$ represents half opening angle of the circular wall segment (Figure 4.2C).

Transmural pressure $p_{\text {Trans }}$ equals total axial force. Consequently, $p_{\text {Trans }}$ is calculated by multiplying $T_{x}$ by the ratio of length of the junction contour $\left(2 \pi y_{m}\right)$ to area of the junction circle $\left(\pi y_{m}{ }^{2}\right)$ :

$$
p_{\text {Trans }}=\frac{2 T_{x}}{y_{m}}
$$

\section{Mechanics of the curved wall patch}

In this section, it is shown how midwall surface area $A_{m}$ and curvature $C_{m}$ are used to calculate representative midwall tension $T_{m}$. For that purpose, a constitutive model of myofiber mechanics is applied. This latter model renders Cauchy myofiber stress from natural myofiber strain and is presented in Appendix 4.B.

In the ventricular module, originally implemented in the CircAdapt model, ${ }^{3}$ the one-fiber model developed by Arts et al. ${ }^{2}$ was used to relate ventricular pump mechanics, as described by cavity pressure and volume, to myofiber mechanics, as described by myofiber stress and strain. When assembling three wall segments to a ventricular composite with two cavities, contractile function of a wall segment, as described by representative midwall tension and area change, should be put 
between mechanics of cavity and myofiber. The following conditions should be satisfied. First, when folding a wall segment to a completely closed spherical surface, the relation between pump mechanics and myofiber mechanics should be equivalent to the equations of the one-fiber model. Second, like in the one-fiber model, where contractile myofiber work equals ventricular pump work, summed pump work of both cavities should be equal to summed work as generated by the three walls. Furthermore, within each wall segment, work as delivered by the wall through wall tension and changes of geometry, should be equal to the work generated by the myofibers.

In Appendix 4.A, it is shown that such a model of wall segment mechanics can be found, although the derivation is quite complex. Because the shape of the midwall area is not relevant for the balance of work, the relations are extended to the more general case of a spherical wall patch with arbitrary shape of the boundary. Thus, for a wall patch it is found that natural myofiber strain $\varepsilon_{f}$ depends on midwall surface area $A_{m}$ and curvature $C_{m}$ by the following approximation (relative error $<1 \%$ ), as derived in Appendix 4.A:

$$
\varepsilon_{f} \approx \frac{1}{2} \ln \left(\frac{A_{m}}{A_{m, \text { ref }}}\right)-\frac{1}{12} z^{2}-0.019 z^{4} \quad \text { with } \quad z=\frac{3 C_{m} V_{w}}{2 A_{m}}
$$

where $A_{m, \text { ref }}$ represents reference midwall surface area. Note that the dimensionless curvature parameter $z$ is closely related to the dimensionless ratio of wall thickness to radius of curvature. With a constitutive model of the myofiber (Appendix 4.B), natural myofiber strain $\varepsilon_{f}$ is used to calculate Cauchy myofiber stress $\sigma_{f}$. In Appendix 4.A, it is derived how myofiber stress and wall segment geometry are used to calculate representative midwall tension $T_{m}$. It holds by approximation (relative error $<2 \%$ ):

$$
T_{m} \approx \frac{V_{w} \sigma_{f}}{2 A_{m}}\left(1+\frac{z^{2}}{3}+\frac{z^{4}}{5}\right) \quad \text { with } \sigma_{f}=f\left(\varepsilon_{f}\right)
$$

Equations 4.15 and 4.16 represent the first few terms of a Taylor series approximating the analytically derived expressions. These approximations have the advantage of being well-defined for zero curvature. The analytical derivations cannot be used in numerical calculations, because zero by zero division occurs near zero curvature, while the ratio is analytically well-defined. 


\section{Implementation of TriSeg model in CircAdapt model}

The TriSeg model of ventricular mechanics is incorporated in the existing CircAdapt model of the whole circulation. ${ }^{3}$ This latter model supplies the required hemodynamic boundary conditions, i.e., LV and RV cavity volumes. The CircAdapt model is designed as a network of modules representing cardiac chambers, valves, large blood vessels, and peripheral resistances. Extended with the TriSeg model, the CircAdapt model allows beat-to-beat simulation of timedependent ventricular mechanics and hemodynamics, e.g., ventricular cavity volumes and pressures, geometries and representative myofiber mechanics of ventricular walls, and flows through valves. An important feature of the existing CircAdapt model is that the number of independent parameters is reduced by incorporating adaptation of cavity size and wall mass of cardiac chambers and blood vessels to mechanical load so that stresses and strains in the walls of heart and blood vessels are normalized to tissue-specific physiological standard levels.

The model was initialized using realistic estimates of wall volume $V_{w}$, reference midwall surface area $A_{m, r e f}$ and reference sarcomere length $L_{s, \text { ref }}$ (Table 4.1: Parameter values for model initialization). After model initialization, these parameters will be obtained for each wall segment by load adaptation as described in the next "Simulations" section. The actual parameter values chosen for initialization (Table 4.1) are noncritical due to load adaptation. However, for fast convergence to steady state adaptation these estimates were chosen in physiological range.

The set of differential equations that describe the CircAdapt model, including the Triseg module, was solved by numerical integration with time steps of 2 ms using the ODE113 function in MATLAB 7.1.0 (MathWorks, Natick, MA). Simulation time of a single cardiac cycle was less than 7 seconds on a Windows XPTM platform (version 2002) with a $2.00 \mathrm{GHz}$ Intel $\left(\right.$ Core ${ }^{\mathrm{TM} 2}$ Duo T7250 processor and 1 GB of RAM. 


\section{Simulations}

The TriSeg model, as integrated in the CircAdapt model, was tested by simulation of human ventricular mechanics and hemodynamics with 1) normal ventricular loading conditions (NORM), 2) acute pulmonary hypertension (PHAc), and 3) chronic pulmonary hypertension including adaptation (PHCh). All simulations are presented with similar values of mean systemic blood flow (cardiac output), cardiac cycle time (heart rate), and mean systemic arterial blood pressure, simulating hemodynamics at rest (Table 4.1: Hemodynamics at rest).

\section{NORM simulation}

The diameter of large blood vessels was assumed to be determined by adaptation to mean chronic circumstances, which state is most closely described by the condition of rest (Table 4.1: Hemodynamics at rest). However, geometry of the heart and wall thickness of large blood vessels were considered to be a result of adaptation to a state of moderate exercise, thus simulating the effect of relatively short periods of training by exercise. The applied simulation protocol of adaptation has been described earlier. ${ }^{3}$ Briefly recapitulating, with resting hemodynamics (Table 4.1: Hemodynamics at rest), diameters of the large blood vessels were adapted until mean blood flow velocity reached the set point value (Table 4.1: Set point values for adaptation). Next, a moderate state of exercise was simulated by tripling cardiac output and doubling heart rate (Table 4.1: Interventions with exercise). Under these circumstances, wall volume $\left(V_{w}\right)$ and reference midwall surface area $\left(A_{m, r e f}\right)$ of all cardiac wall segments were adapted until maximum and minimum sarcomere length as well as maximum passive myofiber stress reached the set point values of adaptation with exercise (Table 4.1). Also, wall thickness of the blood vessels was adapted until maximum wall stress was equal to the set point value (Table 4.1). Next, hemodynamics were returned to rest conditions and the above-mentioned adaptation protocol was repeated two or three times until steady state geometry was reached $(<1 \%$ deviation from set point values). In total, finding the steady state adapted NORM simulation required simulation of about 100-200 cardiac cycles.

\section{PHAc and PHCh simulations}

The NORM simulation was used as point of departure for simulation of ventricular mechanics and hemodynamics with increased pulmonary resistance. Steady state simulations (PHAC1, $\mathrm{PHAC2}$, and $\mathrm{PHAC} 3$ ), representing increasing degrees of acute pulmonary hypertension, were obtained by acute increase of mean pulmonary arteriovenous pressure drop in three steps without adaptation 


\section{Chapter 4}

(Table 4.1: Interventions with PH simulations). Finally, adaptation was applied to the three steady state PHAc simulations following the same protocol used for the NORM simulation. This resulted in three steady state simulations (PHCh1, PHCh2, and PHCh3, respectively), representing gradually increasing degrees of chronic pulmonary hypertension at rest.

Table 4.1. Input parameter values for NORM and PH simulations

\begin{tabular}{|c|c|c|}
\hline Parameter & Unit & Value \\
\hline \multicolumn{3}{|l|}{ Hemodynamics at rest } \\
\hline Mean systemic arterial blood pressure & $\mathrm{kPa}$ & 12.2 \\
\hline Mean systemic blood flow & $\mathrm{m} \cdot \mathrm{s}^{-1}$ & 85 \\
\hline Cardiac cycle time & s & 0.850 \\
\hline Mean pulmonary arteriovenous pressure drop & $\mathrm{kPa}$ & 1.5 (NORM) \\
\hline \multicolumn{3}{|l|}{ Interventions with exercise } \\
\hline Mean systemic blood flow & $\mathrm{m} \cdot \mathrm{s}^{-1}$ & 255 \\
\hline Cardiac cycle time & s & 0.425 \\
\hline \multicolumn{3}{|l|}{ Set point values for adaptation } \\
\hline Mean flow velocity in large blood vessels (rest) & $m \cdot s^{-1}$ & 0.17 \\
\hline Maximum vascular wall stress (exercise) & $\mathrm{kPa}$ & 500 \\
\hline Maximum sarcomere length (exercise) & $\mu \mathrm{m}$ & 2.2 \\
\hline Minimum sarcomere length (exercise) & $\mu \mathrm{m}$ & 1.75 \\
\hline Maximum passive myofiber stress (exercise) & $\mathrm{kPa}$ & 10 (LVfw), 8 (Sept), 20 (RVfw) \\
\hline \multicolumn{3}{|l|}{ Interventions with PH simulations } \\
\hline Mean pulmonary arteriovenous pressure drop & $\mathrm{kPa}$ & $\begin{array}{l}3.0 \text { (PHAc1 and PHCh1), } \\
4.5 \text { (PHAc2 and PHCh2), } \\
6.0 \text { (PHAc3 and PHCh3) }\end{array}$ \\
\hline \multicolumn{3}{|l|}{ Parameter values for model initialization } \\
\hline Wall volume $\left(v_{w}\right)$ & $\mathrm{ml}$ & 75 (LVfw), 40 (Sept), 30 (RVfw) \\
\hline Reference midwall surface area $\left(A_{m, \text { ref }}\right)$ & $\mathrm{cm}^{2}$ & 80 (LVfw), 45 (Sept), 100 (RVfw) \\
\hline Reference sarcomere length $\left(L_{s, \text { ref }}\right)$ & $\mu \mathrm{m}$ & 2.0 (LVfw, Sept, and RVfw) \\
\hline Septal midwall volume $\left(v_{m, \text { Sept }}\right)$ & $\mathrm{ml}$ & 42 \\
\hline Radius of midwall junction circle $\left(y_{m}\right)$ & $\mathrm{cm}$ & 3.3 \\
\hline
\end{tabular}

LVfw, left ventricular free wall; NORM, normal simulation; PHAc, acute pulmonary hypertension simulation; PHCh, chronic pulmonary hypertension simulation; RVfw, right ventricular free wall; Sept, septal wall. 


\section{Simulation data analysis}

\section{Ventricular pump mechanics and hemodynamics}

Simulated time courses of normal (NORM) LV and RV pressures, volumes, and flows were compared with physiological data obtained in normal subjects. ${ }^{26,29}$, 54, 62, 63 Area of ventricular pressure-volume relation was calculated to quantify ventricular pump stroke work $\left(\mathrm{W}_{\text {stroke }}\right)$ for the LV and RV. Time courses of blood flow velocities through the mitral, aortic, tricuspid, and pulmonary valves were used to study the effects of chronic pulmonary hypertension on ventricular hemodynamics. For comparison with clinical data obtained in healthy subjects and in patients with chronic pulmonary hypertension,, 962 several timing parameters of RV hemodynamics were quantified for the NORM and PHCh simulations. RV ejection time (ET) was quantified as the time from pulmonary valve opening to closure, acceleration time of pulmonary flow (AT) as the time from pulmonary valve opening to moment of maximal pulmonary flow velocity, $\mathrm{RV}$ isovolumic contraction time (ICT) as the time from tricuspid valve closure to pulmonary valve opening, and RV isovolumic relaxation time (IRT) as the time from pulmonary valve closure to tricuspid valve opening.

\section{Tissue mechanics}

Area of myofiber stress-strain relation was calculated to quantify stroke work density $\left(w_{\text {stroke }}\right)$ for the LVfw, Sept, and RVfw. Stroke work density was defined as contractile myofiber stroke work per unit of tissue volume.

\section{Ventricular wall geometry}

End-diastolic wall thicknesses $\left(\mathrm{H}_{\mathrm{ed}}\right)$ were calculated as wall volume divided by end-diastolic midwall surface area. End-diastole was defined as the moment of mitral valve closure. Furthermore, time courses of LVfw, Sept, and RVfw midwall curvatures were used to assess acute and chronic effects of increase of pulmonary resistance on wall curvatures. The relation between septal geometry and the transseptal pressure gradient in the TriSeg model was compared with the relation found in patients with and without chronic pulmonary hypertension. ${ }^{17}$ For that purpose, curvature ratio (CR) was calculated as the Sept/LVfw midwall curvature ratio at the moment of aortic valve closure, whereas transmural pressure ratio (PR) was defined as the difference between maximum LV and RV pressures divided by maximum LV pressure. 


\section{RESULTS}

\section{Simulation of normal physiology (NORM)}

Figure 4.4 shows simulated time courses of ventricular cavity pressures and volumes, aortic and pulmonary artery pressures, and flows through arterial and atrioventricular valves under normal ventricular loading conditions (NORM). These time courses show agreement with physiological data on the following aspects: 1) LV and RV cavity pressures and volumes rise at the end of diastole as
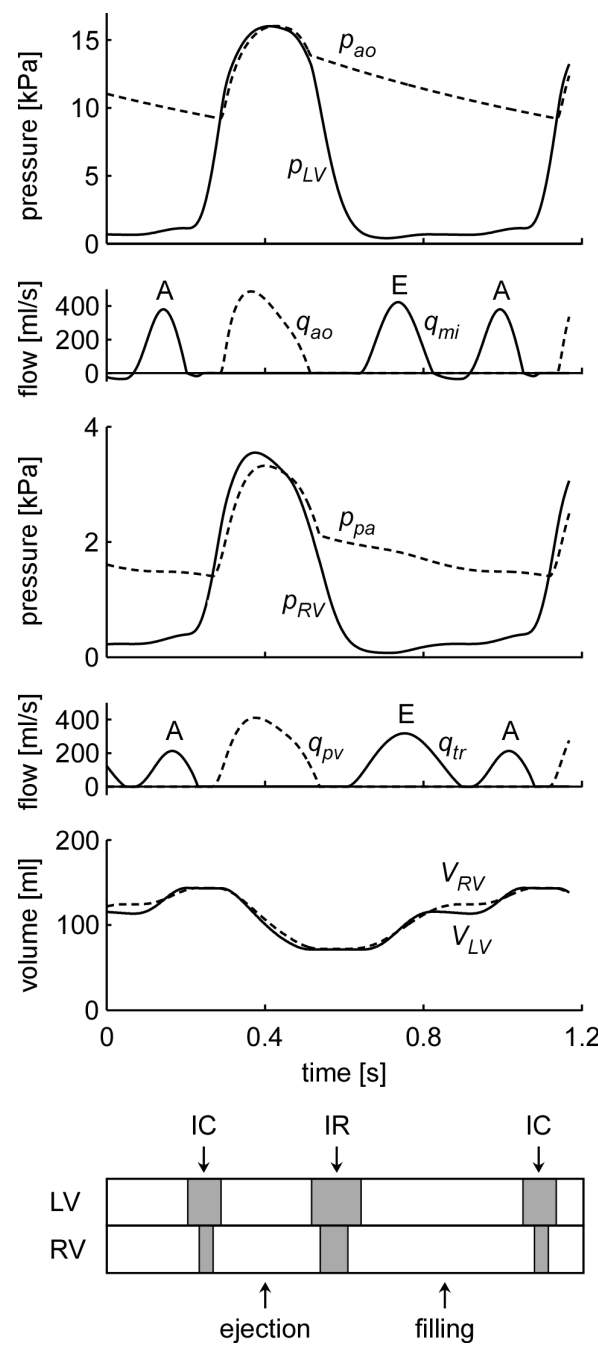

Figure 4.4: Simulated time courses of normal (NORM simulation) LV and RV hemodynamics at rest. The bar plot (bottom panel) displays RV and LV ejection, filling, and isovolumic contraction and relaxation periods. Note that simulation duration amounts to one and a half cardiac cycle. Symbols: A, late active ventricular filling wave; $E$, early passive ventricular filling wave; $I C$, isovolumic contraction; IR, isovolumic relaxation; LV, left ventricular; $p_{a o}$, aortic pressure; $p_{L V}$, left ventricular pressure; $p_{p a}$, pulmonary artery pressure; $p_{R V_{1}}$ right ventricular pressure; $q_{a 0}$, aortic valve flow; $q_{m i}$, mitral valve flow; $q_{p v}$, pulmonary valve flow; $q_{t r}$, tricuspid valve flow; $R V$, right ventricular; $V_{L V}$, left ventricular cavity volume; and $V_{R V}$, right ventricular cavity volume. 
a result of atrial contraction, 2) time courses of mitral and tricuspid valve flows show an early passive filling wave (E) followed by a clearly separated late filling wave (A) resulting from atrial contraction, ${ }^{26} 3$ ) LV and RV end-diastolic volumes are not significantly different, ${ }^{54}$ 4) RV ejection starts earlier and ends later than LV ejection, ${ }^{29}$ and 5) durations of LV isovolumic contraction and relaxation exceed the corresponding RV time intervals (Figure 4.4, bottom panel). ${ }^{62,63}$ Steady state adaptation rendered LVfw, Sept, and RVfw wall volumes of 76, 38, and $31 \mathrm{ml}$, respectively. Wall thicknesses $\mathrm{H}_{\text {ed, Lvfw }}$ and $\mathrm{H}_{\text {ed, Sept }}$ were almost equal and three times as large as $\mathrm{H}_{\text {ed,Rvfw }}$ (Table 4.2).

Table 4.2. Simulation results: ventricular wall thickness

\begin{tabular}{lcccccccc} 
& & NORM & \multicolumn{3}{c}{ PHAc } & \multicolumn{3}{c}{ PHCh } \\
\hline Parameter & Unit & & 1 & 2 & 3 & 1 & 2 & 3 \\
\hline End-diastolic wall thickness & & & & & & & \\
\hline$H_{\text {ed, LVfw }}$ & $\mathrm{mm}$ & 7.9 & 7.8 & 7.6 & 7.3 & 8.3 & 8.2 & 8.3 \\
$\mathrm{H}_{\text {ed, Sept }}$ & $\mathrm{mm}$ & 6.8 & 6.9 & 7.0 & 6.9 & 6.7 & 6.9 & 6.5 \\
$\mathrm{H}_{\text {ed, RVfw }}$ & $\mathrm{mm}$ & 2.3 & 2.1 & 2.0 & 1.7 & 3.3 & 4.2 & 5.1 \\
\hline
\end{tabular}

$H_{\text {ed, }}$, end-diastolic wall thickness; LV, left ventricular; LVfw, left ventricular free wall; NORM, normal simulation; PHAc, acute pulmonary hypertension simulation; PHCh, chronic pulmonary hypertension simulation; RV, right ventricular; RVfw, right ventricular free wall; Sept, septal wall.

\section{Simulations of acute pulmonary hypertension (PHAc)}

The upper panel row of Figure 4.5 illustrates simulated ventricular pressurevolume loops under normal loading conditions (NORM) and with acutely increased pulmonary resistance (PHAc1, PHAc2, and PHAC3). With increasing pulmonary resistance, RV cavity volume, systolic and diastolic RV pressures, mean pulmonary artery pressure, and RV pump work (Table 4.3) increased, while LV cavity volume and pump work were relatively unaffected. The middle panel row of Figure 4.5 shows stress-strain loops of the myofibers in the ventricular wall segments. Acute rise of pulmonary resistance caused increase of RVfw myofiber stress, myofiber strain, and stroke work density, while it caused decrease of LVfw and Sept stroke work densities (Table 4.3). The lower panel row of Figure 4.5 illustrates time courses of LVfw, Sept, and RVfw midwall curvatures. With increasing pulmonary resistance, Sept midwall curvature decreased predominantly during diastole. In the PHAc3 simulation, the septum 
even flattened to zero curvature and bulged shortly towards the LV free wall (negative curvature) during early diastole. LVfw and RVfw curvatures appeared relatively insensitive to acute changes of pulmonary resistance. With increasing pulmonary resistance (Table 4.2), $\mathrm{H}_{\text {ed,Rvfw }}$ decreased maximally by $26 \%$ in PHAc3 relative to NORM, $\mathrm{H}_{\text {ed,Lffw }}$ decreased maximally by $8 \%$, whereas $\mathrm{H}_{\text {ed, Sept }}$ increased only $1 \%$.
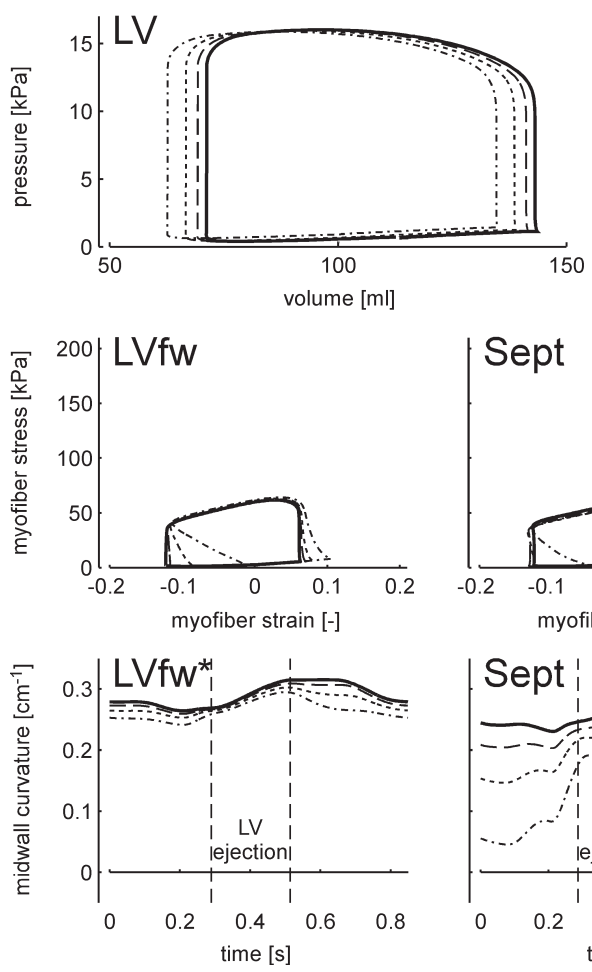
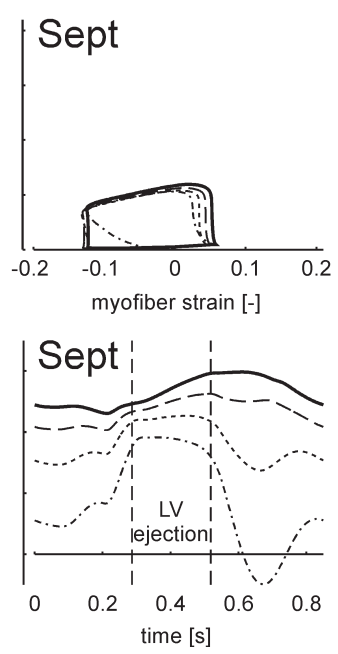
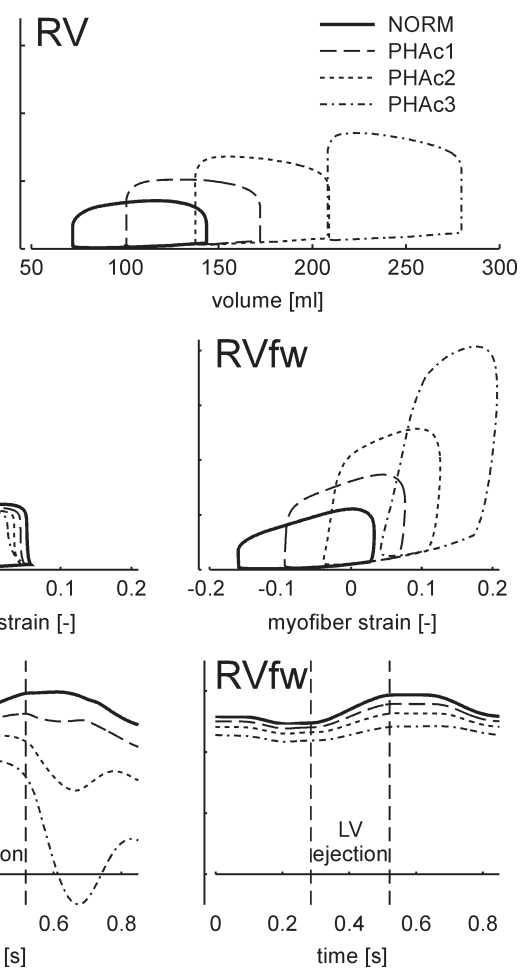

Figure 4.5: Simulated ventricular pressure-volume loops, myofiber stress-strain loops, and time courses of midwall curvatures under normal (NORM) and acute pulmonary hypertensive (PHAC1, PHAC2, and PHAc3) ventricular loading conditions. Acute increase of pulmonary resistance resulted in RV dilatation, increased RV pump work, increased contractile work density in the RV free wall, and decreased septal midwall curvature. Simulation duration amounts to one cardiac cycle. LV ejection is indicated in the curvature plots. Abbreviations: LV, left ventricular; LVfw, left ventricular free wall; RV, right ventricular; RVfw, right ventricular free wall; and Sept, septal wall.

* Note that the absolute value of LVfw midwall curvature is used for graphical representation, while this curvature is defined to be negative in the TriSeg model. 
Table 4.3. Simulation results: pulmonary artery pressures and ventricular work

\begin{tabular}{|c|c|c|c|c|c|c|c|c|}
\hline \multirow{2}{*}{ Parameter } & \multirow[b]{2}{*}{ Unit } & \multirow[t]{2}{*}{ NORM } & \multicolumn{3}{|c|}{ PHAC } & \multicolumn{3}{|c|}{ PHCh } \\
\hline & & & 1 & 2 & 3 & 1 & 2 & 3 \\
\hline \multicolumn{9}{|c|}{ Ventricular pump stroke work } \\
\hline$W_{\text {stroke, LV }}$ & $\mathrm{Pa} \cdot \mathrm{m}^{3}$ & 1.04 & 1.04 & 1.03 & 1.02 & 1.05 & 1.04 & 1.04 \\
\hline $\mathrm{W}_{\text {stroke, } \mathrm{RV}}$ & $\mathrm{Pa} \cdot \mathrm{m}^{3}$ & 0.22 & 0.32 & 0.42 & 0.51 & 0.35 & 0.48 & 0.61 \\
\hline \multicolumn{9}{|c|}{ Stroke work density } \\
\hline $\mathrm{W}_{\text {stroke, } \mathrm{LVfw}}$ & $\mathrm{kPa}$ & 9.41 & 9.54 & 9.32 & 8.66 & 8.88 & 8.90 & 8.78 \\
\hline$W_{\text {stroke, Sept }}$ & $\mathrm{kPa}$ & 8.94 & 8.28 & 7.54 & 6.15 & 9.19 & 8.66 & 8.49 \\
\hline $\mathrm{W}_{\text {stroke, } \mathrm{RVfw}}$ & $\mathrm{kPa}$ & 7.91 & 11.66 & 15.94 & 21.59 & 8.59 & 9.13 & 9.48 \\
\hline \multicolumn{9}{|c|}{ Mean pulmonary artery pressure } \\
\hline$p_{p a}$ & $\mathrm{kPa}$ & 2.04 & 3.56 & 5.11 & 6.77 & 3.53 & 5.00 & 6.47 \\
\hline
\end{tabular}

LV, left ventricular; LVfw, left ventricular free wall; NORM, normal simulation; PHAc, acute pulmonary hypertension simulation; $\mathrm{PHCh}$, chronic pulmonary hypertension simulation; $\mathrm{p}_{\mathrm{pa}}$, mean pulmonary artery pressure; RV, right ventricular; RVfw, right ventricular free wall; Sept, septal wall; $\mathrm{W}_{\text {stroke, }}$ stroke work density; $\mathrm{W}_{\text {stroke, }}$ ventricular pump stroke work.

\section{Simulations of chronic pulmonary hypertension (PHCh)}

Figure 4.6 illustrates graphs of the same variables as presented in Figure 4.5, but now rendered after adaptation to increased pulmonary resistance (PHCh1, PHCh2, and PHCh3). Like in absence of adaptation, systolic and diastolic RV pressures, mean pulmonary artery pressure, and RV pump work were elevated with chronic pulmonary hypertension (Table 4.3), while LV pump function remained relatively unaffected. In contrast to the PHAc simulations, RV cavity volume decreased $8 \%$ in the PHCh3 simulation (Figure 4.6). The myofiber stressstrain loops in Figure 4.6 show that adaptation resulted in a more homogeneous distribution of stroke work density in the ventricular walls (Table 4.3). In the PHCh3 simulation, RVfw stroke work density was increased (20\%), whereas LVfw and Sept stroke work densities were decreased (7\% and 5\%, respectively) relative to the NORM simulation. Increase of pulmonary resistance with load adaptation resulted in the following changes of ventricular geometry. Mean Sept curvature (Figure 4.6) decreased linearly with increasing pulmonary resistance, while the shape of the time course was preserved. LVfw and RVfw curvatures were practically unaffected. Relative to the NORM simulation, $\mathrm{H}_{\text {ed, Rvfw }}$ increased up to $120 \%$ in PHCh3, whereas changes of $\mathrm{H}_{\text {ed, Lvfw }}$ and $\mathrm{H}_{\text {ed,Sept }}$ were small $(<5 \%)$ (Table 4.2). 
In Figure 4.7, time courses of LV and RV inflow and outflow velocities are shown for the NORM and PHCh simulations. With increasing pulmonary resistance, pulmonary and tricuspid valve flow velocities changed significantly relative to the NORM simulation, whereas aortic and mitral valve flow velocities remained relatively unaffected. Furthermore, in Figure 4.7, RV ejection time (ET),
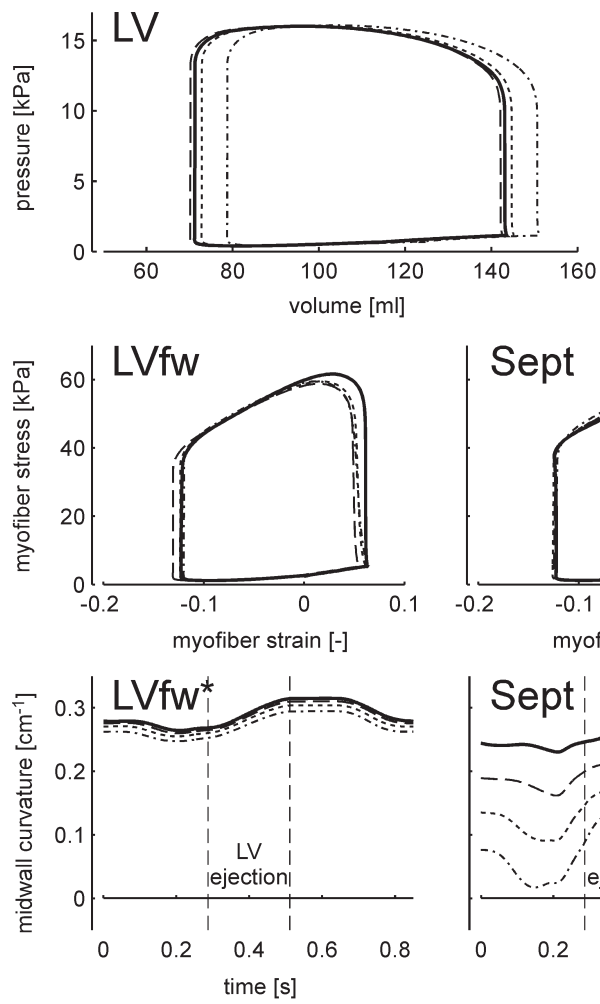
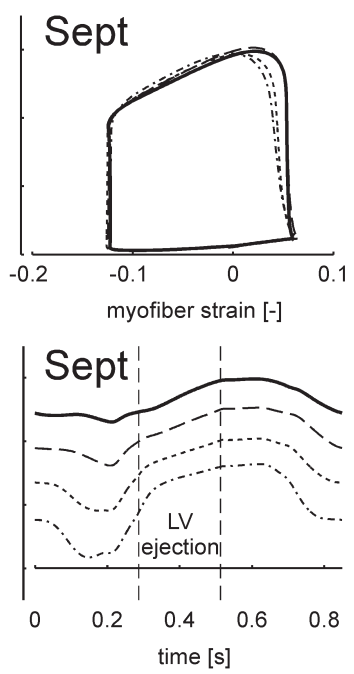
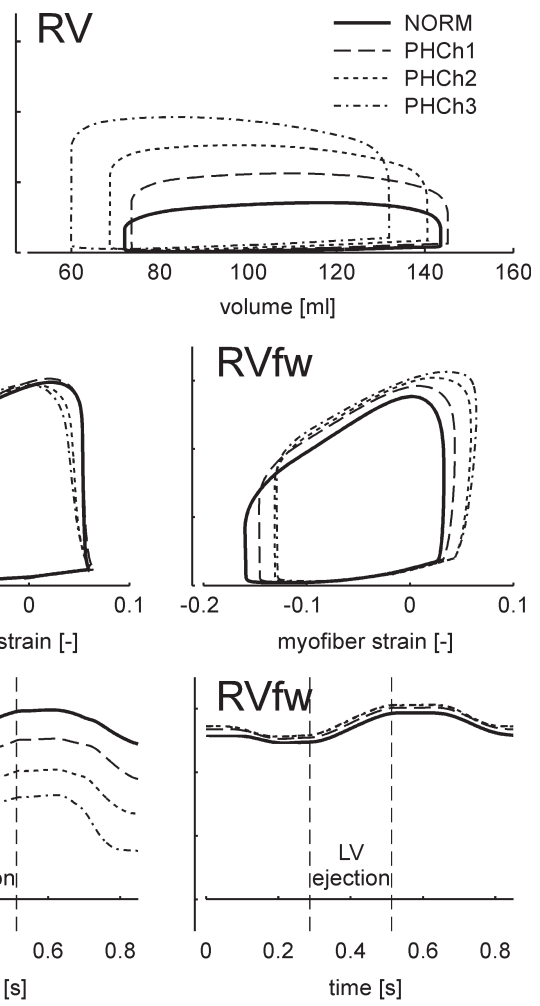

Figure 4.6: Simulated ventricular pressure-volume loops, myofiber stress-strain loops, and time courses of midwall curvatures under normal (NORM) and chronic pulmonary hypertensive (PHCh1, $\mathrm{PHCh} 2$, and $\mathrm{PHCh} 3$ ) ventricular loading conditions. Chronic increase of pulmonary resistance including adaptation resulted in increase of RV pump work, homogeneity of contractile work densities in the walls, and linear decrease of septal midwall curvature. Simulation duration amounts to one cardiac cycle. LV ejection is indicated in the curvature plots. Abbreviations: LV, left ventricular; LVfw, left ventricular free wall; RV, right ventricular; RVfw, right ventricular free wall; and Sept, septal wall.

* Note that the absolute value of LVfw midwall curvature is used for graphical representation, while this curvature is defined to be negative in the TriSeg model. 
acceleration time of pulmonary flow (AT), RV isovolumic contraction time (ICT), and RV isovolumic relaxation time (IRT) are indicated for the NORM simulation. In Figure 4.8, changes of these timing parameters are compared with the changes as observed in clinical studies among normal individuals and patients with chronic pulmonary hypertension. ${ }^{9,62}$ In the simulations, chronic increase of pulmonary resistance caused decrease of AT and AT/ET-ratio, indicating increase of pulmonary flow curve skewness. This is in accordance with measurements as obtained in patients with chronic pulmonary hypertension. ${ }^{9}$ Furthermore, simulated ICT and IRT lengthened (maximally $163 \%$ and $47 \%$ in the PHCh3 simulation, respectively). The ratio of total RV isovolumic time (ICT+IRT) to ET almost doubled. Again, the simulated changes were similar to the changes as observed in patients with pulmonary hypertension. ${ }^{62}$ Furthermore, the tricuspid early filling wave narrowed and the tricuspid E/A-ratio of peak atrioventricular flow during early filling $(E)$ to that during atrial contraction $(A)$ decreased from 1.49 in the NORM simulation to unity in the PHCh3 simulation.
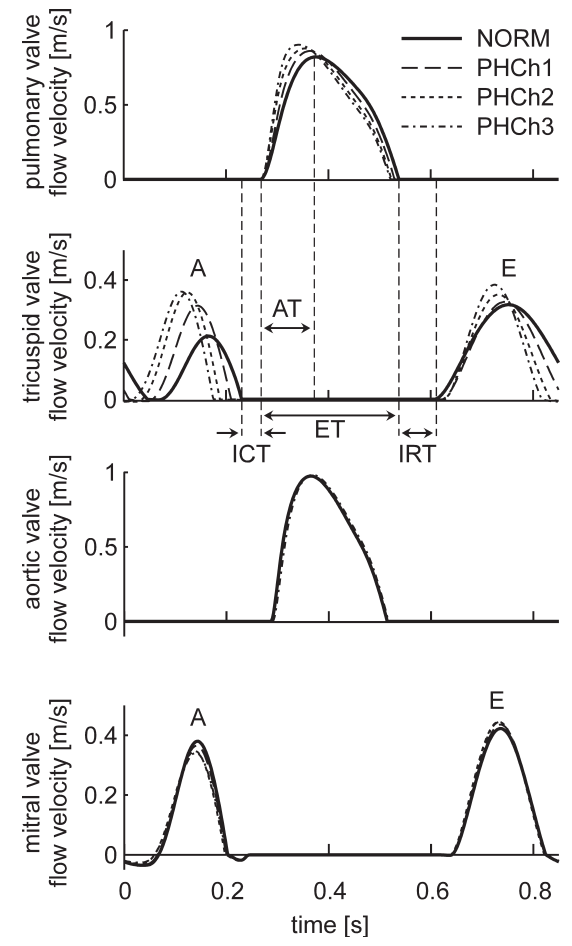

Figure 4.7: Simulated time courses of pulmonary and tricuspid valve flow velocities (upper two panels) show lengthening of RV isovolumic contraction, shortening of RV ejection, and increase of pulmonary flow acceleration after adaptation to increased pulmonary resistance (PHCh1, $\mathrm{PHCh} 2$, and PHCh3). Aortic and mitral valve flow velocities (lower two panels) appear relatively insensitive to increase of pulmonary resistance. Simulation duration amounts to one cardiac cycle. The following time intervals are indicated for the NORM simulation: AT, acceleration time of pulmonary valve flow; ET, RV ejection time; ICT, RV isovolumic contraction time; and IRT, RV isovolumic relaxation time. Symbols: A, late active ventricular filling wave; and $E$, early passive ventricular filling wave. 

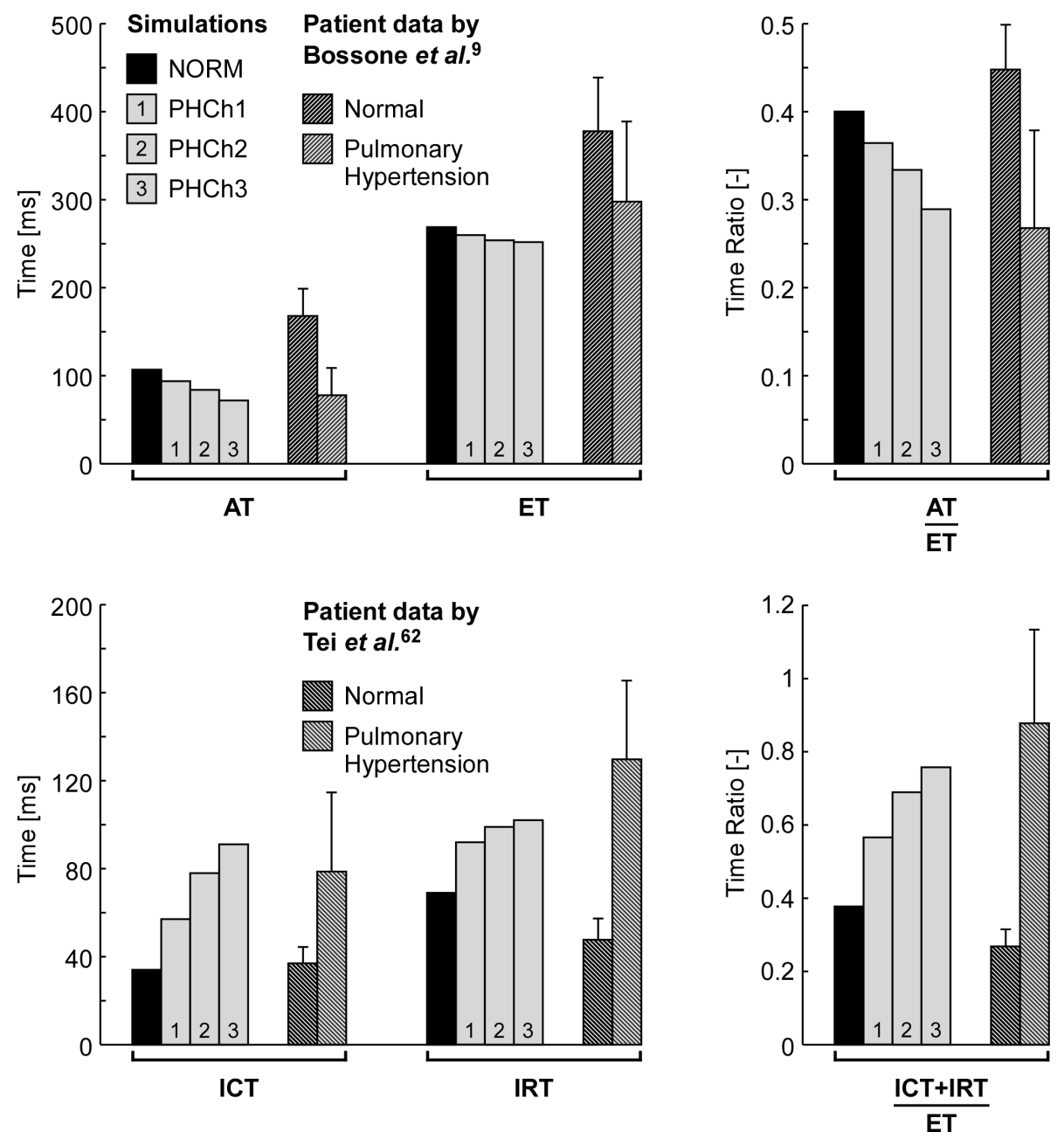

Figure 4.8: Comparison of simulated and patient data on timing of RV hemodynamics. Simulated changes of AT, ET, ICT, and IRT as chronic response to increased pulmonary resistance agree with measurements obtained in healthy normals and patients with pulmonary hypertension [Adapted from Bossone et al. ${ }^{9}$ (upper row) and Tei et al. ${ }^{62}$ (lower row)]. Patient data are presented by mean values with standard deviations indicated by error bars. Symbols: AT, pulmonary flow acceleration time; ET, RV ejection time; ICT, RV isovolumic contraction time; and IRT, RV isovolumic relaxation time. 


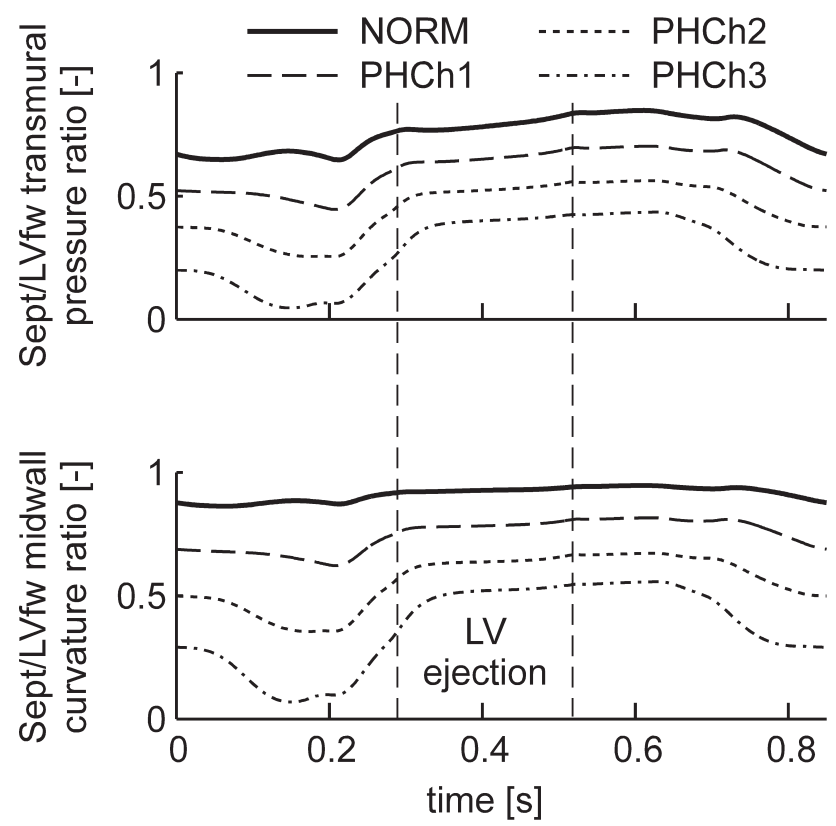

Figure 4.9: Simulated time courses of Sept/LVfw transmural pressure ratio (upper panel) and Sept/LVfw midwall curvature ratio (lower panel) appear similar revealing a linear relation between both ratios at each time point in the cardiac cycle. Simulation duration amounts to one cardiac cycle. Duration of LV ejection is indicated by vertical dashed lines.

Figure 4.9 shows simulated time courses of Sept/LVfw transmural pressure ratio and Sept/LVfw midwall curvature ratio. Sept transmural pressure was quantified as LV cavity pressure minus RV cavity pressure, while LVfw transmural pressure was quantified as LV cavity pressure. At each time point in the cardiac cycle, both ratios decreased linearly with increase of pulmonary resistance. 
Both CR and PR decreased with increase of pulmonary resistance (Table 4.4). Figure 4.10 shows the relation between $C R$ and $P R$, as derived from the NORM and PHCh simulations, as well as the relation found among patients with and without pulmonary hypertension. ${ }^{17}$ In this latter patient population, pulmonary hypertension was defined as an RV systolic pressure $>40 \mathrm{mmHg}$. Linear

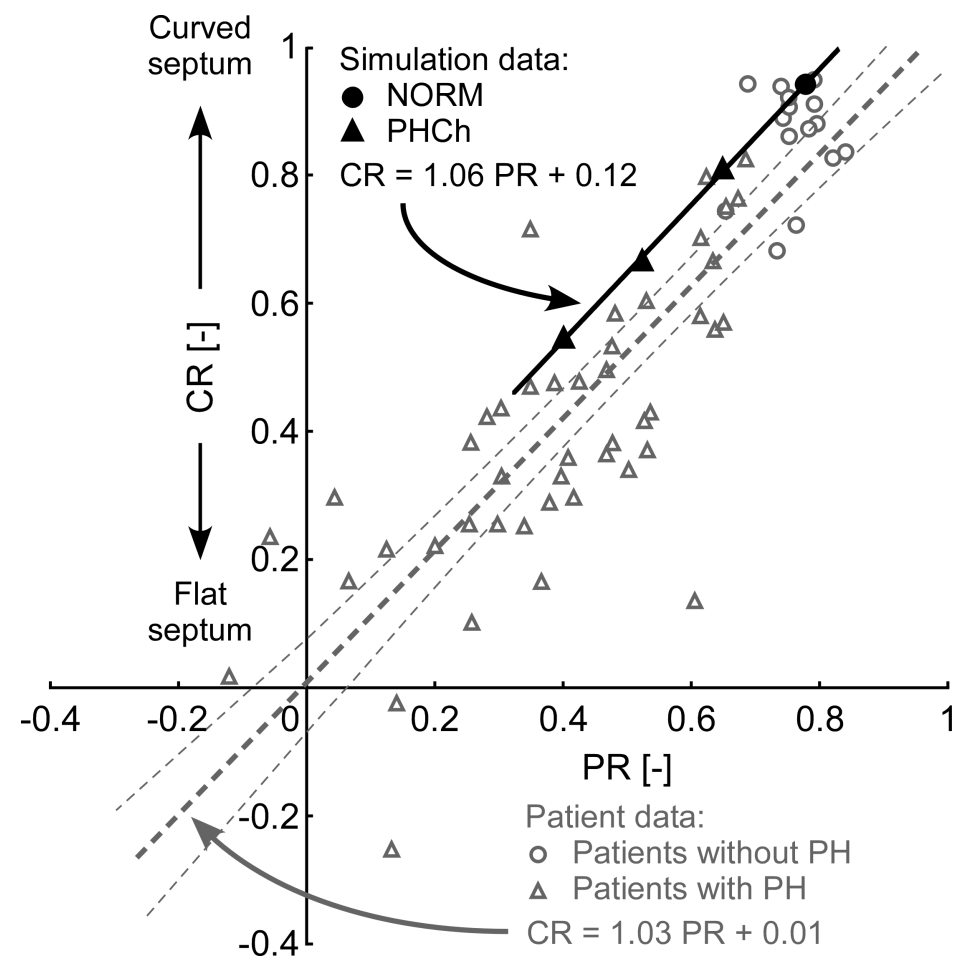

Figure 4.10: Simulation results on the correlation between curvature ratio $C R$ and transmural pressure ratio PR (black data points), see text for definitions, as compared with the relation extracted from data (grey data points) obtained in patients with and without pulmonary hypertension ( $\mathrm{PH}=\mathrm{RV}$ systolic pressure $>40 \mathrm{mmHg}$ ) [Adapted from Dellegrottaglie et al. ${ }^{17}$ ]. Linear regression analysis of data points as derived from the NORM and PHCh simulations revealed a strong correlation (bold black line, $r^{2}=1.00, S E E=0.008$ ) between $C R$ and $P R$ with similar slope as the relation found in patients (thick grey dashed line, $r^{2}=0.73, S E E=0.044$ ). The thin dashed lines indicate the $95 \%$ confidence interval of linear regression fit to the patient data. Linear regression formulas are shown for simulated as well as measured data points. Symbols: r, regression residual; and SEE, standard error of the estimate. 
regression analysis of data points as derived from the NORM and PHCh simulations revealed a strong correlation $\left(r^{2}=1.00, S E E=0.008\right)$ between $C R$ and PR with similar slope as the relation found in patients $\left(r^{2}=0.73, S E E=0.044\right)$. In the simulations, however, an offset was found, shifting the relation to higher $C R$ values (Figure 4.10). It is noted (not shown) that although acute change $( \pm 20 \%)$ of mean systemic flow or mean arterial blood pressure affected CR and PR, their interdependence followed the linear relation as derived from the chronic simulations within $2 \%$ deviation. The same holds for the data points derived from the PHAC1 and PHAC2 simulations, while for the PHAC3 simulation the deviation was more than $10 \%$ (Table 4.4, not shown in Figure 4.10).

Table 4.4. Simulation results: curvature ratio and transmural pressure ratio

\begin{tabular}{lcccccccc} 
& & NORM & \multicolumn{3}{c}{ PHAC } & \multicolumn{3}{c}{ PHCh } \\
\hline Parameter & Unit & & 1 & 2 & 3 & 1 & 2 & 3 \\
\hline CR & - & 0.94 & 0.85 & 0.72 & 0.54 & 0.81 & 0.67 & 0.55 \\
PR & - & 0.78 & 0.68 & 0.57 & 0.46 & 0.65 & 0.52 & 0.40 \\
\hline
\end{tabular}

$C R$, septal-to-LV free wall midwall curvature ratio at the moment of aortic valve closure; NORM, normal simulation; PHAc, acute pulmonary hypertension simulation; PHCh, chronic pulmonary hypertension simulation; PR, systolic septal-to-LV free wall transmural pressure ratio. 


\section{Discussion}

The newly designed TriSeg model of ventricular mechanics incorporates mechanical interaction of the LV free wall, RV free wall, and septal wall resulting in a strong coupling of LV and RV pump mechanics and hemodynamics. The TriSeg model was successfully implemented as a module in the available CircAdapt model, simulating cardiac mechanics and hemodynamics of the closed-loop circulation. Effects of ventricular interaction on cardiac mechanics and hemodynamics were assessed by simulation of pulmonary hypertension in the acute phase as well as in the chronic phase, the latter implying adaptation of ventricular geometry to mechanical load. For chronic pulmonary hypertension, simulated ventricular geometry, hemodynamics, and septal mechanics agreed surprisingly well with corresponding measurements in patients.

\section{Model assumptions}

In the TriSeg model, ventricular geometry was approximated by three thickwalled spherical segments encapsulating the LV and RV cavities (Figure 4.2A). In reality, the ventricular cavities are enclosed by truncated ellipsoidal muscular walls and the non-contractile basal sheet with valves. ${ }^{58}$ The simplification to spherical segments without a non-contractile sheet resulted in a general underestimation of ventricular dimensions. Although the fact that there are indications that bending stiffness of the myocardium may be important for simulation of septal geometry, ${ }^{7,21}$ we neglected this effect for simplicity. Despite these inaccuracies, relative changes of dimensions during the cardiac cycle as a result of adaptation were simulated realistically.

In the TriSeg model, myofiber strain was estimated from midwall curvature, area, and wall volume by application of the one-fiber mode ${ }^{2}$ to a spherical wall segment (Eq. 4.15 and Appendix 4.A). The one-fiber model has been shown to be insensitive to actual wall geometry by assuming conservation of energy and homogeneity of fiber stress in the wall. ${ }^{2}$ So, we expected that the present relation for transmural pressure as a function of midwall surface area and curvature was also applicable to the real, more irregular cardiac geometry, although this fact has not been proven.

The analytically derived dependency of myofiber strain on wall segment geometry (Eq. 4.A7) appeared continuous and differentiable around $z=0$. To avoid numerical inaccuracy near zero curvature, because of zero-division, a fourth-order Taylor series approximation (Eq. 4.15) was used instead. For similar reasons, Eq. 4.A4 for midwall tension was also approximated by a fourth-order 
Taylor series (Eq. 4.16). Within the physiological range of ventricular geometry, the errors of the approximations as compared to the analytically derived relations were smaller than $1 \%$ and $2 \%$ for strain and tension, respectively. With respect to total ventricular pump work, total myofiber stroke work was overestimated less than $2.6 \%$, whereas total tensile stroke work at the midwall surface was underestimated less than $0.5 \%$.

In the NORM and PHCh simulations, a load-controlling adaptation mechanism was applied to render size and mass of each wall segment. The applied adaptation rules required prescribed values for maximum and minimum sarcomere lengths and for maximum passive myofiber stress in each wall segment (Table 4.1). Maximum and minimum sarcomere lengths were derived from experiments on isolated cardiac muscle of the rat. ${ }^{15,31,64}$ Maximum passive myofiber stress was chosen as adaptation stimulus because experimental data obtained in dogs with chronic volume overload suggested that end-diastolic myofiber stress and ejection strain were important mechanical stimuli for hypertrophy, while peak systolic myofiber stress appeared irrelevant. ${ }^{22}$ However, when assuming similar values of maximum passive myofiber stress in the three ventricular walls of the TriSeg model, ventricular geometry did not develop anatomically accurate. Therefore, in our model, we adjusted levels of maximum diastolic stress per wall segment (Table 4.1) so that after adaptation the weight ratios for the walls agreed with findings in healthy volunteers. 19,28

In the present study, the external pressure surrounding the LV and RV free walls was assumed to be zero. The real heart is surrounded by the pericardium, which constrains increase of total heart volume during volume overload. Under resting conditions, the pericardium is believed to play a minor role, setting pericardial pressure close to zero. ${ }^{42,46,67}$ However, with acute increase of total heart volume, the pericardium affects cardiac hemodynamics and interaction of the cardiac chambers significantly. ${ }^{6,11,25}$ In our simulations of acute pulmonary hypertension (PHAC), the effect of the pericardium should be considered because of severe RV dilatation (Figure 4.5). In the PHAc3 simulation, RV cavity volume even increased by $120 \%$ relative to the NORM simulation. Together with the changes of right atrial and LV cavity volumes (45\% increase and 10\% decrease, respectively), total heart volume increased by $25 \%$. In the chronic PH simulations, the effect of the pericardium on ventricular mechanics is likely to be of minor importance, because measurements demonstrated adaptive dilatation of the pericardium in patients with chronic pulmonary hypertension. ${ }^{8,24}$ 


\section{Comparison of model simulations with measurements}

The relation between curvature ratio $C R$ and transmural pressure ratio $P R$ as extracted from the NORM and PHCh simulations (Figure 4.10) agreed quite well with the relation as found in a patient group consisting of patients with and without chronic pulmonary hypertension. ${ }^{17}$ The relations were about linear with equal slope. In the simulations, however, an offset was found, shifting the relation to higher CR values, implying overestimation of septal curvature for a given right-to-left pressure ratio.

The overestimation of septal curvature may have many causes. In the model, wall geometry was considered spherical having a clear unique radius of curvature by definition. The real ventricular walls are not spherical, ${ }^{58}$ implying that radii of curvature along the circumferential and base-to-apex direction are different. Also, the junction of the real ventricular walls is smoothed over the wall boundaries, thus further hindering a clear definition of curvature. Besides an error in the curvature ratio as derived from the simulations, the offset might originate from a systematic error in the pressure or curvature measurements obtained in patients as discussed by Dellegrottaglie et al. ${ }^{17}$ Nonetheless, the linear relationship between CR and PR might be a useful tool for noninvasive estimation of systolic RV cavity pressure in patients with RV pressure overload. ${ }^{17,35,51}$ In the simulations, the linear relation appeared also valid $(<2 \%$ deviation) for the PHAc1 and PHAc2 simulations (Table 4.4) as well as after acute changes $( \pm 20 \%)$ of mean arterial pressure and cardiac output in the chronic pulmonary hypertension simulations (not shown in Figure 4.10). The latter findings suggested that the linear relation between $C R$ and $P R$ is insensitive to acute changes of hemodynamic status.

Experimental studies with acute manipulation of transseptal pressure difference as well as clinical studies among patients with chronic pulmonary hypertension showed that septal curvature and position of the septum between the LV and RV free walls depend instantaneously on transseptal pressure difference. 10,20,21,35,36,52 This dependency was found during systole as well as diastole. Similar dependencies were found in our simulations of pulmonary hypertension. Septal curvature decreased with increase of pulmonary resistance (Figures 4.5 and 4.6). Furthermore, leftward shift of the septum, increase of RV volume, and decrease of $\mathrm{LV}$ volume with acute increase of pulmonary resistance (Figure 4.5), as predicted by the TriSeg model, were also predicted by Kerckhoffs et al. ${ }^{34}$ using a finite element model of the ventricles coupled to a lumped circulation model.

Simulations and clinical observations were in agreement concerning 
changes in timing of cardiac flow events due to pulmonary hypertension (Figures 4.7 and 4.8). For example, acceleration time of pulmonary flow velocity was significantly decreased and varied linearly with mean pulmonary artery pressure. ${ }^{9,14}$ Furthermore, RV isovolumic contraction and relaxation times increased while pulmonary ejection time decreased. ${ }^{62,70}$ Moreover, tricuspid E/Aratio was decreased in patients with chronic pulmonary hypertension indicating deterioration of RV diastolic function. ${ }^{71}$

Ventricular wall volumes and end-diastolic wall thicknesses (Table 4.2) in the NORM simulation were all about $25 \%$ smaller than values measured in healthy volunteers. ${ }^{19,28,57}$ This difference most likely resulted from an overestimation of contractility used in the sarcomere mechanics model (Appendix 4.B, Eq. 4.B7), causing walls to be thinner after adaptation to mechanical load. Furthermore, pulmonary acceleration and ejection times as derived from the NORM simulation (Figure 4.8) were underestimated as compared to data obtained in healthy individuals. ${ }^{9,27}$ A probable cause is inaccuracy in the model description of sarcomere mechanics, which is primarily derived from experiments on isolated cardiac muscle of the rat. ${ }^{15,31,64}$ Human myocardium under in vivo conditions is likely to behave differently.

In the PHCh simulations, LV pump function and hemodynamics were relatively unaffected by increase of pulmonary resistance (Figures 4.6 and 4.7 ). Also in rats with chronic pulmonary hypertension, resting LV pump function remains unaffected as long as the myocardium is able to compensate for increased tissue load by structural adaptation. ${ }^{23}$ Furthermore, in the PHCh simulations, the RV free wall hypertrophied and RV cavity volume decreased (Table 4.2 and Figure 4.6). These geometric changes were in agreement with experimental observations in rats with mild chronic pulmonary hypertension. ${ }^{49}$ In the latter study, chronic pulmonary artery banding resulted in an increase of $\mathrm{RV}$ systolic pressure from 33 to $71 \mathrm{mmHg}$. This increase of RV afterload resulted in $76 \%$ increase of thickness of the RV free wall and 14\% decrease of RV free wall area, which suggests a small reduction in RV cavity volume. In our simulations, RV systolic pressure increased from $27 \mathrm{mmHg}$ in the NORM simulation to $72 \mathrm{mmHg}$ in the PHCh3 simulation (Figure 4.6), end-diastolic wall thickness increased $120 \%$ (Table 4.2), and RV cavity volume decreased $8 \%$. In another animal study in rats, ${ }^{44}$ it was shown that structural adaptation to chronic increase of RV afterload was associated with concentric hypertrophy up to a certain level. Beyond this level, however, the myocardium could not fully compensate for further load increase. Consequently, the RV cavity dilated, mainly due to dilatation of the RV free wall. In patients with severe chronic 
pulmonary hypertension, RV free wall hypertrophy is found to occur together with RV dilatation. ${ }^{12,30}$ Secondary to RV failure, systolic and diastolic LV function deteriorate by ventricular interaction. ${ }^{38,39}$ As a result, cardiac performance deteriorates and exercise capacity reduces. ${ }^{12,45}$ These phenomena indicating RV failure were also found in our PHAc simulations.

In the PHAc simulations, the myocardial tissue was unable to compensate for increased RV afterload by load adaptation. As a result, mechanical myofiber load was inhomogeneously distributed over the ventricular walls (Table 4.3 and Figure 4.5). RVfw stroke work density increased with increase of RV afterload, whereas LVfw and Sept stroke work densities decreased. In the PHAc3 simulation, RVfw stroke work density was increased by almost $200 \%$ with respect to the NORM simulation. When assuming stroke work density to be correlated to oxygen consumption, ${ }^{59}$ this implies an increase of oxygen demand by the RV free wall. Since oxygen supply is not included in our model, the potential effect of perfusion imposed limitations on myocardial performance is unknown in our simulations of pulmonary hypertension.

In patients with pulmonary hypertension, tricuspid regurgitation often occurs due to RV and tricuspid annular dilatation. ${ }^{18,65}$ In our simulations, tricuspid valve regurgitation was not included. In the PHAc3 simulation, RV enddiastolic volume was increased by almost $100 \%$ with respect to the NORM simulation. It is likely that the absence of tricuspid regurgitation resulted in underestimation of RV volume overload in the PHAc simulations.

The TriSeg model was successfully integrated as a module in the CircAdapt model of the closed-loop cardiovascular system. As shown previously, the CircAdapt environment is flexible by its modular setup and enables realistic simulation of cardiovascular mechanics and hemodynamics under normal as well as various pathological conditions. ${ }^{3,33,40}$ A set of physiological adaptation rules, expressing structural adaptation of the system to mechanical load, makes the model self-structuring and reduces the number of independent model parameters. For each ventricular wall segment in the TriSeg model, wall volume, midwall area, and reference sarcomere length were varied so that mechanical myofiber load was normalized to a known physiological level, which was assumed to be the same for all ventricular walls. The field of application of the CircAdapt model was substantially enlarged by implementation of the TriSeg model. In the future, the combined model may be used to study fundamental research questions concerning ventricular interaction and its role in cardiac pathologies. For example, specific material properties may be changed per wall segment in order to study effects of heterogeneity of wall properties on cardiac 
mechanics and hemodynamics, e.g., asynchronous mechanical activation (and pacing) or a localized myocardial infarct. 33,40

Although the TriSeg model can be easily modified to include a description of inhomogeneous myocardial wall properties, a finite element model of cardiac mechanics is more accurate and better suited to describe local inhomogeneities in mechanical load. For patient-specific modeling of the circulation, the model should simulate many cycles in order to find a best match with the available set of measurements. For that purpose, the TriSeg model is to be preferred because calculation effort was about 1,000 times less than that of a finite element approach. The finite element model is absolutely needed to estimate and evaluate possible errors introduced by the applied simplifications of the TriSeg model. Currently, the CircAdapt model with the TriSeg module will be evaluated in its possibility to simulate hemodynamics of pulmonary hypertension patient-specifically.

\section{Conclusions}

We presented the Triseg model of ventricular mechanics and hemodynamics incorporating mechanical interaction of the LV free wall, RV free wall, and septal wall, resulting in a strong coupling of LV and RV hemodynamics. The model enables calculation of LV and RV pressures given the respective cavity volumes. LV and RV hemodynamics are related to myofiber mechanics in the three ventricular walls, satisfying the principle of conservation of energy. The three ventricular walls are mechanically coupled satisfying equilibrium of tensile forces in their junction. After implementation as a module in the lumped closed-loop CircAdapt model of heart and circulation, the TriSeg model enables simulation of ventricular hemodynamics and wall mechanics as functions of time.

Simulations of cardiac mechanics and hemodynamics during normal ventricular loading, acute pulmonary hypertension, and chronic pulmonary hypertension after load adaptation agreed with clinical data as obtained in normal subjects and in chronic pulmonary hypertension patients. With increasing levels of chronic pulmonary hypertension, the TriSeg model predicted increase of systolic pulmonary flow acceleration, increase of isovolumic contraction and relaxation times, and linear decrease of septal-to-LV free wall curvature ratio. Summarizing, the TriSeg model realistically describes ventricular mechanics including the interaction between left and right ventricular pump mechanics, dynamics of septal geometry, and contractile myofiber function in the three ventricular walls. 


\section{ACKNOWLEDGMENTS}

We gratefully acknowledge the financial support of Actelion Pharmaceuticals Nederland B.V. (Woerden, The Netherlands) and the Netherlands Heart Foundation Grant 2007B203.

\section{Appendix 4.A: Derivations of mechanics of the CURVed wall patch}

This appendix contains the analytical derivations of Eqs. 4.15 and 4.16 relating mechanics of the curved wall patch to myofiber mechanics. For the derivation of Eq. 4.16, calculating representative midwall tension $T_{m}$ from myofiber stress, the logarithmic one-fiber model relating global ventricular pump mechanics to myofiber mechanics is used. ${ }^{2}$ Applying the definition of midwall surface as used in Eqs. 4.3 and 4.4 , for the ratio of transmural pressure $p_{\text {Trans }}$ to myofiber stress $\sigma_{f}$ in a closed thick-walled sphere with midwall volume $V_{m}$ and wall volume $V_{w}$, it holds:

$$
\frac{p_{\text {Trans }}}{\sigma_{f}}=\left.\frac{1}{3} \ln \left(\frac{1+z}{1-z}\right)\right|_{\text {sphere }} \quad \text { with } \quad z=\left.\frac{V_{w}}{2 V_{m}}\right|_{\text {sphere }}
$$

where the definition of $z$ is equivalent to the more general definition used in Eq. 4.15 for a curved wall patch. For a closed sphere with midwall surface area $A_{m}$ and curvature $C_{m}$, the relation between $p_{\text {Trans }}$ and representative midwall tension $T_{m}$ is determined by conservation of energy, i.e.,

$$
p_{\text {Trans }} d V_{m}=\left.T_{m} d A_{m}\right|_{\text {sphere }}
$$

where $d V_{m}$ and $d A_{m}$ represent small increments of midwall volume and area, respectively. Substitution of $V_{m}=4 / 3 \pi r^{3}, A_{m}=4 \pi r^{2}$, and $r=1 / C_{m}$ in $E q .4 . A 2$ yields:

$$
p_{\text {Trans }}=2 T_{m} C_{m}
$$

Note that, Eq. 4.A3 represents Laplace's law expressed in such a way that the applied scalar definition of $T_{m}$ satisfies conservation of energy (Eq. 4.A2). Although we have derived Eq. 4.A3 for a complete sphere, it also holds for a fraction of the same sphere, since both curvature $C_{m}$ and tension $T_{m}$ are local parameters. Thus, Eq. 4.A3 may also be applied to a spherical wall segment (Figure 4.2C). Substituting Eq. 4.A3 in Eq. $4 . A 1$ and applying $C_{m}=\left(2 A_{m} z\right) /\left(3 V_{w}\right)$ renders for representative midwall tension $T_{m}$ in a curved wall patch: 


$$
T_{m}=\frac{\sigma_{f} V_{w}}{2 A_{m}} \cdot \frac{1}{2 z} \ln \left(\frac{1+z}{1-z}\right)
$$

Note that this latter function is continuous around zero curvature. To avoid numerical inaccuracy near $z=0, E q .4 . A 4$ is replaced by its close fourth-order polynomial approximation as specified in Eq. 4.16 (relative error $<2 \%$ ).

For the derivation of Eq. 4.15, myofiber strain $\varepsilon_{f}$ is considered a function of two variables, i.e., $A_{m}$ and $C_{m}$. Applying the principle of conservation of energy, work of the wall by a small area change $d A_{m}$ with tension $T_{m}$ equals work of the myofibers by a change of natural myofiber strain $d \varepsilon_{f}$ with Cauchy myofiber stress $\sigma_{f}$ :

$$
T_{m} d A_{m}=V_{w} \sigma_{f} d \varepsilon_{f}
$$

Rearranging Eq. 4.A5, substituting $\mathrm{Tm}$ with Eq. 4.A4, and keeping $C_{m}$ constant renders the following partial derivative:

$$
\frac{\partial \varepsilon_{f}}{\partial A_{m}}=\frac{1}{4 A_{m} z} \ln \left(\frac{1+z}{1-z}\right) \quad \text { with } \quad z=\frac{3 C_{m} V_{w}}{2 A_{m}}
$$

A standard mathematical solution technique is used to find the general solution of the first-order partial differential equation in Eq. 4.A6. ${ }^{16}$ For that purpose, $\varepsilon_{f}$ is written as the sum of a strain term $\varepsilon_{f, A C}$ and a strain term $\varepsilon_{f, C}$ :

$$
\varepsilon_{f}=\varepsilon_{f, A C}\left(A_{m}, C_{m}\right)+\varepsilon_{f, C}\left(C_{m}\right)
$$

First, integration of $E q$. $4 . A 6$ with respect to $A_{m}$ renders strain term $\varepsilon_{f, A C}$ under the condition that $C_{m}$ remains constant during integration:

$$
\varepsilon_{f, A C}\left(A_{m}, C_{m}\right)=\frac{1}{2} \ln \left(\frac{A_{m}}{A_{m, \text { ref }}}\right)+\frac{1}{4 z} \ln \left(\frac{(1+z)^{1+z}}{(1-z)^{1-z}}\right)
$$

Symbol $A_{m, r e f}$ represents a reference value of midwall surface area $A_{m}$. Below, the term $\varepsilon_{f, C}$ will be derived for a relatively simple special case of inflating a closed sphere. The thus found expression is valid for all combinations of $A_{m}$ and $C_{m}$. For a closed thick-walled sphere myofiber strain $\varepsilon_{f}$ is known as a function of $V_{m}$. Using the principle that work as generated by the myofibers equals pump work, it holds: 


$$
V_{w} \sigma_{f} d \varepsilon_{f}=\left.p_{\text {Trans }} d V_{m}\right|_{\text {sphere }}
$$

Substitution of Eq. 4.A1 in Eq. 4.A9 renders for a closed sphere:

$$
\frac{d \varepsilon_{f}}{d V_{m}}=\left.\frac{1}{3 V_{w}} \ln \left(\frac{1+z}{1-z}\right)\right|_{\text {sphere }}
$$

Then, midwall volume $V_{m}$ and curvature ratio $z$ are expressed as functions of curvature $C_{m}$ :

$$
\begin{aligned}
& V_{m}=\left.\frac{4 \pi}{3 C_{m}^{3}}\right|_{\text {sphere }} \\
& z=\left.\frac{3 V_{w} C_{m}^{3}}{8 \pi}\right|_{\text {sphere }}
\end{aligned}
$$

Substitution of Eqs. 4.A11 and 4.A12 in Eq. 4.A10 results in a differential equation in $C_{m}$ only. Integration of this differential equation with respect to $C_{m}$ yields an expression of myofiber strain $\varepsilon_{f}$ for a closed sphere as function of $C_{m}$ (full equation given in Appendix 4.C):

$$
\varepsilon_{f}=\left.f\left(C_{m}\right)\right|_{\text {sphere }}
$$

Applying Eq. 4.A8 to a closed sphere with curvature $C_{m}$ and substituting the resulting expression as well as Eq. 4.A13 in Eq. 4.A7, results in the unknown strain term $\varepsilon_{f, C}$ (full equation given in Appendix 4.C):

$$
\varepsilon_{f, C}\left(C_{m}\right)=\left.\varepsilon_{f}\right|_{\text {sphere }}-\left.\varepsilon_{f, A C}\left(A_{m}, C_{m}\right)\right|_{\text {sphere }}
$$

Finally, substitution of Eqs. 4.A8 and 4.A14 in Eq. $4 . A 7$ yields a complicated analytical expression of natural myofiber strain $\varepsilon_{f}$ in a curved wall patch (Appendix 4.C). Mathematica 5.2 (Wolfram Research, Inc) was used to obtain a simpler fourth-order polynomial fit as specified in Eq. 4.15, This latter expression closely approximates the analytical derivation (relative error $<1 \%$ ). 


\section{Appendix 4.B: Model of Myofiber Mechanics}

In this appendix, the applied heuristic relation is presented that converts natural myofiber strain $\varepsilon_{f}$ to Cauchy myofiber stress $\sigma_{f}$. This empirical representation of sarcomere contraction simulates experiments on isolated rat cardiac muscle. ${ }^{15,64}$ Parameter values are given in Table 4.B.

Natural myofiber strain is converted to sarcomere length $L_{s}$ by:

$$
L_{s}=L_{s, \text { ref }} \mathrm{e}^{\varepsilon_{f}}
$$

The sarcomere has been modeled as a passive element in parallel with a series combination of a contractile element and series elastic element. The timedependent behavior is described by two state variables, i.e., contractile element length $L_{s c}$ and mechanical activation $C$. This latter activation parameter is physiologically related to intracellular calcium concentration. The time derivative of $L_{s c}$ depends linearly on length of the series elastic element $\left(L_{s}-L_{s c}\right)$ and equals zero for isometric contraction:

$$
\frac{d L_{s c}}{d t}=\left(\frac{L_{s}-L_{s c}}{L_{s e, s o}}-1\right) v_{\max }
$$

where $v_{\max }$ represents velocity of sarcomere shortening with zero load and $L_{s, \text { iso }}$ length of the isometrically stressed series elastic element (Table 4.B). Dependence on $v_{\max }$ represents the myofiber force-velocity relation so that shortening velocity increases with applied external force.

The time derivative of $C$ is heuristically obtained, having separate terms to describe rise and decay of mechanical activation $C$ :

$$
\frac{d C}{d t}=\frac{1}{\tau_{R}} \cdot C_{L}\left(L_{s c}\right) \cdot F_{\text {rise }}(t)+\frac{1}{\tau_{D}} \cdot \frac{C_{\text {rest }}-C}{1+\mathrm{e}^{\left(T\left(L_{s c}\right)-t\right) / \tau_{D}}}
$$


Parameters $\tau_{R}$ and $\tau_{D}$ are scaling rise and decay time, respectively (Table 4.B). Symbols $t$ and $C_{\text {rest }}$ represent time and diastolic resting level of activation, respectively. Functions $C_{L}, F_{\text {rise, }}$ and $T$ describe increase of activation with sarcomere length, rise of mechanical activation, and decrease of activation duration with decrease of sarcomere length, respectively:

$$
\begin{aligned}
& C_{L}\left(L_{s c}\right)=\tanh \left(4.0\left(L_{s c}-L_{s c 0}\right)^{2}\right) \\
& F_{\text {rise }}(t)=0.02 \cdot x^{3}(8-x)^{2} \mathrm{e}^{-x} \\
& \text { with } x=\min \left(8, \max \left(0, t / \tau_{R}\right)\right) \\
& T\left(L_{s c}\right)=\tau_{s c}\left(0.29+0.3 L_{s c}\right)
\end{aligned}
$$

where $L_{s c o}$ and $\tau_{s c}$ represent contractile element length with zero load and a time factor scaling duration of contraction, respectively (Table 4.B). and $C$ :

Active myofiber stress $\sigma_{f, \text { act }}$ depends on length of series elastic element, $L_{s c}$

$$
\sigma_{f, \text { act }}=\sigma_{a c t} \cdot C \cdot\left(L_{s c}-L_{s c o}\right) \cdot \frac{L_{s}-L_{s c}}{L_{s e, i s o}}
$$

In order to simulate the contributions of titin as well as collagen to passive myocardial stiffness, ${ }^{69}$ passive stress $\sigma_{f, \text { pas }}$ represents a soft behavior for low myofiber strain and a stiff behavior for large positive strain:

$$
\sigma_{t, \text { pas }}=\sigma_{\text {pas }}\left(36 \cdot \max \left(0, \varepsilon_{f}-0.1\right)^{2}+0.1\left(\varepsilon_{f}-0.1\right)+0.0025 \mathrm{e}^{30 \varepsilon_{f}}\right)
$$

The value of $\sigma_{\text {pas }}$ has been adapted so that at maximum sarcomere length with exercise, passive myofiber stress assumes the maximum value (Table 4.1: Set point values for adaptation). Total Cauchy myofiber stress $\sigma_{f}$ is the sum of active and passive stress:

$$
\sigma_{f}\left(\varepsilon_{f}\right)=\sigma_{f, \text { pas }}+\sigma_{f, \text { act }}
$$

The latter result has been substituted in Eq. 4.16. 
Table 4.B. Parameter values of the sarcomere mechanics model

\begin{tabular}{llcl} 
Symbol & Unit & Value & Parameter description \\
\hline$C_{\text {rest }}$ & - & 0.02 & Diastolic resting level of activation \\
$L_{s c 0}$ & $\mu \mathrm{m}$ & 1.51 & Contractile element length with zero active stress \\
$L_{\text {s,ref }}$ & $\mu \mathrm{m}$ & 2.0 & Reference sarcomere length at zero strain \\
$L_{\text {se,iso }}$ & $\mu \mathrm{m}$ & 0.04 & Length of isometrically stressed series elastic element \\
$V_{\max }$ & $\mu \mathrm{m} \cdot \mathrm{s}^{-1}$ & 7 & Sarcomere shortening velocity with zero load \\
$T_{D}$ & $\mathrm{~ms}$ & 32 & Factor scaling contraction decay time \\
$T_{R}$ & $\mathrm{~ms}$ & 48 & Factor scaling contraction rise time \\
$T_{\text {sc }}$ & $\mathrm{ms}$ & 425 & Factor scaling duration of contraction \\
$\sigma_{\text {act }}$ & $\mathrm{kPa}$ & 120 & Factor scaling active myofiber stress \\
$\sigma_{\text {pas }}$ & $\mathrm{kPa}$ & 7 & Factor scaling passive myofiber stress
\end{tabular}

\section{Appendix 4.C: Polynomial series approximations}

The polynomial series approximation given by Eq. 4.15 is obtained from the analytical solution expressing natural myofiber strain in terms of $A_{m}$ and $C_{m}$ (Eq. 4.A7) by applying the following steps:

1. Expression of myofiber strain $\varepsilon_{f}$ for a closed sphere (use $A_{m}=4 \pi / C_{m}{ }^{2}$ ) as function of $C_{m}$ (Eq. 4.A13) yields:

$$
\begin{aligned}
& \left.\varepsilon_{f}\right|_{\text {sphere }}=-\ln \left(C_{m}\right)+\frac{\ln (8 \pi)}{3}+\frac{G(x)}{6} \\
& G(x)=\frac{1}{x} \cdot \ln \left(\frac{(1+x)^{1+x}}{(1-x)^{1-x}}\right) \\
& x=\left.z\right|_{\text {sphere }}=\frac{3 C_{m}^{3} V_{w}}{8 \pi}
\end{aligned}
$$




\section{Chapter 4}

2. Applying Eq. 4.A8 to a closed sphere (use $A_{m}=4 \pi / C_{m}^{2}$ ) yields:

$$
\left.\varepsilon_{f, A C}\right|_{\text {sphere }}=-\ln \left(C_{m}\right)+\frac{1}{2} \cdot \ln \left(\frac{4 \pi}{A_{m, \text { ref }}}\right)+\frac{G(x)}{4}
$$

Substitution of Eqs. 4.C1 and 4.C2 in Eq. 4.A14 yields for strain term $\varepsilon_{f, c}$ :

$$
\varepsilon_{f, C}=\frac{\ln (8 \pi)}{3}-\frac{1}{2} \cdot \ln \left(\frac{4 \pi}{A_{m, \text { ref }}}\right)-\frac{G(x)}{12}
$$

The thus found expression for $\varepsilon_{t, C}$, which holds for the special case of inflating a sphere, is valid for all combinations of $A_{m}$ and $C_{m}$.

3. Substitution of Eqs. 4.C3 and 4.A8 in Eq. 4.A7 yields the general analytical solution of natural myofiber strain $\varepsilon_{f}$ in a curved wall patch:

$$
\varepsilon_{f}=\frac{\ln (8 \pi)}{3}-\frac{1}{2} \cdot \ln \left(\frac{4 \pi}{A_{m, \text { ref }}}\right)+\frac{1}{2} \cdot \ln \left(\frac{A_{m}}{A_{m, \text { ref }}}\right)-\frac{G(x)}{12}+\frac{G(z)}{4}
$$

4. In order to facilitate series approximation, $z=K / A$ and $x=K^{3}$ are substituted in Eq. 4.C4:

$$
\begin{aligned}
\varepsilon_{f}= & \frac{\ln (8 \pi)}{3}-\frac{1}{2} \cdot \ln \left(\frac{4 \pi}{A_{m, \text { ref }}}\right)+\frac{1}{2} \cdot \ln \left(\frac{A_{m}}{A_{m, \text { ref }}}\right) \\
& -\frac{1}{12 K^{3}} \cdot \ln \left(\frac{\left(1+K^{3}\right)^{1+K^{3}}}{\left(1-K^{3}\right)^{1-K^{3}}}\right)+\frac{A}{4 K} \cdot \ln \left(\frac{\left(1+\frac{K}{A}\right)^{1+\frac{K}{A}}}{\left(1-\frac{K}{A}\right)^{1-\frac{K}{A}}}\right)
\end{aligned}
$$

with $A$ and $K$ representing normalized area and curvature, respectively:

$$
\begin{aligned}
& A=\frac{A_{m}}{\sqrt[3]{9 \pi V_{w}^{2}}} \\
& K=\frac{C_{m}}{2} \sqrt[3]{\frac{3 V_{w}}{\pi}}
\end{aligned}
$$


5. The first two terms of Eq. 4.C5 are constant strain offsets and are therefore eliminated from the strain relation, which describes the transition from a reference to a new state with state variables $A_{m}$ and $C_{m}$. Taylor series approximation of this simplified version of Eq. 4.C5 yields:

$$
\varepsilon_{f}=\frac{1}{2} \cdot \ln \left(\frac{A_{m}}{A_{m, \text { ref }}}\right)-\frac{K^{2}}{12 A^{2}}-\frac{K^{4}}{40 A^{4}}+O\left(K^{6}\right)
$$

where $O\left(K^{6}\right)$ represents the higher order terms.

6. It is used that $z=K / A$. Furthermore, the fourth-order polynomial approximation of natural myofiber strain in a curved wall patch (Eq. 4.15) is obtained after slight adjustment of the fourth-order term of Eq. 4.C6 for best accuracy within the whole range of operation $(|z|<0.8)$.

The polynomial series approximation given by Eq. 4.16 is obtained from the analytical solution expressing representative midwall tension in terms of $A_{m}, C_{m}$, and $\sigma_{f}(E q .4 . A 4)$ by applying the following steps:

1. Substitution of $z=\left(3 C_{m} V_{w}\right) /\left(2 A_{m}\right)$ in Eq. 4.A4 yields:

$$
\frac{T_{m}}{\sigma_{f}}=\frac{1}{6 C_{m}} \ln \left(\frac{2 A_{m}+3 C_{m} V_{w}}{2 A_{m}-3 C_{m} V_{w}}\right)
$$

2. Similarly to what has been done to obtain Eq. 4.C5, $A$ and $K$ are substituted in Eq. 4.C7 to facilitate series approximation:

$$
\frac{T_{m}}{\sigma_{f}}=\frac{L}{6 K} \ln \left(\frac{1+\frac{K}{A}}{1-\frac{K}{A}}\right)
$$

with

$$
L=\frac{1}{2} \sqrt[3]{\frac{3 V_{w}}{\pi}}
$$


3. Taylor series approximation of Eq. 4. C8 yields:

$$
\frac{T_{m}}{\sigma_{f}}=\frac{L}{3 A}+\frac{L K^{2}}{9 A^{3}}+\frac{L K^{4}}{15 A^{5}}+O\left(K^{6}\right)
$$

where $O\left(K^{6}\right)$ represents the higher order terms, which are neglected in the Triseg model.

4. Finally, the fourth-order polynomial approximation of representative midwall tension (Eq. 4.16), is obtained by rearranging Eq. 4.C9 and using $z=K / A$. 


\section{REFERENCES}

1. Arts T, Bovendeerd P, Delhaas T, and Prinzen F. Modeling the relation between cardiac pump function and myofiber mechanics. J Biomech 36: 731-736, 2003.

2. Arts T, Bovendeerd PH, Prinzen FW, and Reneman RS. Relation between left ventricular cavity pressure and volume and systolic fiber stress and strain in the wall. Biophys J 59: 93-102, 1991.

3. Arts T, Delhaas T, Bovendeerd P, Verbeek X, and Prinzen FW. Adaptation to mechanical load determines shape and properties of heart and circulation: The circadapt model. Am J Physiol Heart Circ Physiol 288: H1943-1954, 2005.

4. Arts T, Prinzen FW, Snoeckx LH, Rijcken JM, and Reneman RS. Adaptation of cardiac structure by mechanical feedback in the environment of the cell: A model study. Biophys J 66: 953-961, 1994.

5. Baker AE, Dani R, Smith ER, Tyberg JV, and Belenkie I. Quantitative assessment of independent contributions of pericardium and septum to direct ventricular interaction. Am J Physiol 275: H476-483, 1998.

6. Belenkie I, Dani R, Smith ER, and Tyberg JV. The importance of pericardial constraint in experimental pulmonary embolism and volume loading. Am Heart J 123: 733-742, 1992.

7. Beyar R, Dong SJ, Smith ER, Belenkie I, and Tyberg JV. Ventricular interaction and septal deformation: A model compared with experimental data. Am J Physiol 265: H20442056, 1993.

8. Blanchard DG, and Dittrich HC. Pericardial adaptation in severe chronic pulmonary hypertension. An intraoperative transesophageal echocardiographic study. Circulation 85: 1414-1422, 1992.

9. Bossone E, Avelar E, Bach DS, Gillespie B, Rubenfire M, and Armstrong WF. Diagnostic value of resting tricuspid regurgitation velocity and right ventricular ejection flow parameters for the detection of exercise induced pulmonary arterial hypertension. Int J Card Imaging 16: 429-436, 2000.

10. Brinker JA, Weiss JL, Lappe DL, Rabson JL, Summer WR, Permutt S, and Weisfeldt ML. Leftward septal displacement during right ventricular loading in man. Circulation 61: 626-633, 1980.

11. Calvin JE. Optimal right ventricular filling pressures and the role of pericardial constraint in right ventricular infarction in dogs. Circulation 84: 852-861, 1991.

12. Chemla D, Castelain V, Herve P, Lecarpentier $Y$, and Brimioulle S. Haemodynamic evaluation of pulmonary hypertension. Eur Respir J 20: 1314-1331, 2002.

13. Chung DC, Niranjan SC, Clark JW, Jr., Bidani A, Johnston WE, Zwischenberger JB, and Traber DL. A dynamic model of ventricular interaction and pericardial influence. Am J Physiol 272: H2942-2962, 1997. 


\section{Chapter 4}

14. Dabestani A, Mahan G, Gardin JM, Takenaka K, Burn C, Allfie A, and Henry WL. Evaluation of pulmonary artery pressure and resistance by pulsed doppler echocardiography. Am J Cardiol 59: 662-668, 1987.

15. de Tombe PP, and ter Keurs HE. Force and velocity of sarcomere shortening in trabeculae from rat heart. Effects of temperature. Circ Res 66: 1239-1254, 1990.

16. Debnath L. Nonlinear partial differential equations for scientists and engineers. Boston: Birkhäuser, 2005, p. 737.

17. Dellegrottaglie S, Sanz J, Poon M, Viles-Gonzalez JF, Sulica R, Goyenechea M, Macaluso F, Fuster V, and Rajagopalan S. Pulmonary hypertension: Accuracy of detection with left ventricular septal-to-free wall curvature ratio measured at cardiac mr. Radiology 243: 63-69, 2007.

18. Dittrich HC, McCann HA, and Blanchard DG. Cardiac structure and function in chronic thromboembolic pulmonary hypertension. Am J Card Imaging 8: 18-27, 1994.

19. Doherty NE, 3rd, Fujita N, Caputo GR, and Higgins CB. Measurement of right ventricular mass in normal and dilated cardiomyopathic ventricles using cine magnetic resonance imaging. Am J Cardiol 69: 1223-1228, 1992.

20. Dong SJ, Crawley AP, MacGregor JH, Petrank YF, Bergman DW, Belenkie I, Smith ER, Tyberg JV, and Beyar R. Regional left ventricular systolic function in relation to the cavity geometry in patients with chronic right ventricular pressure overload. A threedimensional tagged magnetic resonance imaging study. Circulation 91: 2359-2370, 1995.

21. Dong SJ, Smith ER, and Tyberg JV. Changes in the radius of curvature of the ventricular septum at end diastole during pulmonary arterial and aortic constrictions in the dog. Circulation 86: 1280-1290, 1992.

22. Donker DW, Volders PG, Arts T, Bekkers BC, Hofstra L, Spatjens RL, Beekman JD, Borgers $\mathrm{M}, \mathrm{Crijns} \mathrm{HJ}$, and Vos MA. End-diastolic myofiber stress and ejection strain increase with ventricular volume overload--serial in-vivo analyses in dogs with complete atrioventricular block. Basic Res Cardiol 100: 372-382, 2005.

23. Faber MJ, Dalinghaus M, Lankhuizen IM, Steendijk P, Hop WC, Schoemaker RG, Duncker DJ, Lamers JM, and Helbing WA. Right and left ventricular function after chronic pulmonary artery banding in rats assessed with biventricular pressure-volume loops. Am J Physiol Heart Circ Physiol 291: H1580-1586, 2006.

24. Freeman $\mathrm{GL}$, and LeWinter MM. Pericardial adaptations during chronic cardiac dilation in dogs. Circ Res 54: 294-300, 1984.

25. Freeman $\mathrm{GL}$, and LeWinter MM. Role of parietal pericardium in acute, severe mitral regurgitation in dogs. Am J Cardiol 54: 217-219, 1984.

26. Fujii J, Yazaki Y, Sawada H, Aizawa T, Watanabe H, and Kato K. Noninvasive assessment of left and right ventricular filling in myocardial infarction with a two-dimensional doppler echocardiographic method. J Am Coll Cardiol 5: 1155-1160, 1985. 
27. Gardin JM, Burn CS, Childs WJ, and Henry WL. Evaluation of blood flow velocity in the ascending aorta and main pulmonary artery of normal subjects by doppler echocardiography. Am Heart J 107: 310-319, 1984.

28. Hajduczok ZD, Weiss RM, Stanford W, and Marcus ML. Determination of right ventricular mass in humans and dogs with ultrafast cardiac computed tomography. Circulation 82: 202-212, 1990.

29. Hirschfeld S, Meyer R, Schwartz DC, Korfhagen J, and Kaplan S. Measurement of right and left ventricular systolic time intervals by echocardiography. Circulation 51: 304-309, 1975.

30. Horan LG, Flowers NC, and Havelda CJ. Relation between right ventricular mass and cavity size: An analysis of 1500 human hearts. Circulation 64: 135-138, 1981.

31. Hunter PJ, McCulloch AD, and ter Keurs HE. Modelling the mechanical properties of cardiac muscle. Prog Biophys Mol Biol 69: 289-331, 1998.

32. Kaul S. The interventricular septum in health and disease. Am Heart J 112: 568-581, 1986.

33. Kerckhoffs RC, Lumens J, Vernooy K, Omens JH, Mulligan LJ, Delhaas T, Arts T, McCulloch AD, and Prinzen FW. Cardiac resynchronization: Insight from experimental and computational models. Prog Biophys Mol Biol 97: 543-561, 2008.

34. Kerckhoffs RC, Neal ML, Gu Q, Bassingthwaighte JB, Omens JH, and McCulloch AD. Coupling of a $3 \mathrm{~d}$ finite element model of cardiac ventricular mechanics to lumped systems models of the systemic and pulmonic circulation. Ann Biomed Eng 35: 1-18, 2007.

35. King ME, Braun H, Goldblatt A, Liberthson R, and Weyman AE. Interventricular septal configuration as a predictor of right ventricular systolic hypertension in children: A crosssectional echocardiographic study. Circulation 68: 68-75, 1983.

36. Kingma I, Tyberg JV, and Smith ER. Effects of diastolic transseptal pressure gradient on ventricular septal position and motion. Circulation 68: 1304-1314, 1983.

37. Little WC, Reeves RC, Arciniegas J, Katholi RE, and Rogers EW. Mechanism of abnormal inter-ventricular septal motion during delayed left ventricular activation. Circulation 65: 1486-1491, 1982.

38. Louie EK, Lin SS, Reynertson SI, Brundage BH, Levitsky S, and Rich S. Pressure and volume loading of the right ventricle have opposite effects on left ventricular ejection fraction. Circulation 92: 819-824, 1995.

39. Louie EK, Rich S, Levitsky S, and Brundage BH. Doppler echocardiographic demonstration of the differential effects of right ventricular pressure and volume overload on left ventricular geometry and filling. J Am Coll Cardiol 19: 84-90, 1992.

40. Lumens J, Delhaas T, Kirn B, and Arts T. Modeling ventricular interaction: A multiscale approach from sarcomere mechanics to cardiovascular system hemodynamics. Pac Symp Biocomput 378-389, 2008. 


\section{Chapter 4}

41. Luo C, Ware DL, Zwischenberger JB, and Clark JW, Jr. Using a human cardiopulmonary model to study and predict normal and diseased ventricular mechanics, septal interaction, and atrio-ventricular blood flow patterns. Cardiovasc Eng 7: 17-31, 2007.

42. Mangano DT, Van Dyke DC, Hickey RF, and Ellis RJ. Significance of the pericardium in human subjects: Effects on left ventricular volume, pressure and ejection. J Am Coll Cardiol 6: 290-295, 1985.

43. Maughan WL, Sunagawa K, and Sagawa K. Ventricular systolic interdependence: Volume elastance model in isolated canine hearts. Am J Physiol 253: H1381-1390, 1987.

44. Minami S, Onodera T, Okazaki F, Miyazaki H, Ohsawa S, and Mochizuki S. Myocyte morphological characteristics differ between the phases of pulmonary hypertensioninduced ventricular hypertrophy and failure. Int Heart J 47: 629-637, 2006.

45. Miyamoto S, Nagaya N, Satoh T, Kyotani S, Sakamaki F, Fujita M, Nakanishi N, and Miyatake K. Clinical correlates and prognostic significance of six-minute walk test in patients with primary pulmonary hypertension. Comparison with cardiopulmonary exercise testing. Am J Respir Crit Care Med 161: 487-492, 2000.

46. Morris-Thurgood JA, and Frenneaux MP. Diastolic ventricular interaction and ventricular diastolic filling. Heart Fail Rev 5: 307-323, 2000.

47. Nash MP, and Hunter PJ. Computational mechanics of the heart: From tissue structure to ventricular function. J Elasticity 61: 113-141, 2000.

48. Olansen JB, Clark JW, Khoury D, Ghorbel F, and Bidani A. A closed-loop model of the canine cardiovascular system that includes ventricular interaction. Comput Biomed Res 33: 260-295, 2000.

49. Olivetti G, Ricci R, Lagrasta C, Maniga E, Sonnenblick EH, and Anversa P. Cellular basis of wall remodeling in long-term pressure overload-induced right ventricular hypertrophy in rats. Circ Res 63: 648-657, 1988.

50. Olsen CO, Tyson GS, Maier GW, Spratt JA, Davis JW, and Rankin JS. Dynamic ventricular interaction in the conscious dog. Circ Res 52: 85-104, 1983.

51. Reisner SA, Azzam Z, Halmann M, Rinkevich D, Sideman S, Markiewicz W, and Beyar R. Septal/free wall curvature ratio: A noninvasive index of pulmonary arterial pressure. J Am Soc Echocardiogr 7: 27-35, 1994.

52. Roeleveld RJ, Marcus JT, Faes TJ, Gan TJ, Boonstra A, Postmus PE, and Vonk-Noordegraaf A. Interventricular septal configuration at mr imaging and pulmonary arterial pressure in pulmonary hypertension. Radiology 234: 710-717, 2005.

53. Santamore WP, and Burkhoff D. Hemodynamic consequences of ventricular interaction as assessed by model analysis. Am J Physiol 260: H146-157, 1991.

54. Sechtem U, Pflugfelder PW, Gould RG, Cassidy MM, and Higgins CB. Measurement of right and left ventricular volumes in healthy individuals with cine mr imaging. Radiology 163: 697-702, 1987. 
55. Slinker BK, and Glantz SA. End-systolic and end-diastolic ventricular interaction. Am J Physiol 251: H1062-1075, 1986.

56. Smith BW, Chase JG, Shaw GM, and Nokes RI. Simulating transient ventricular interaction using a minimal cardiovascular system model. Physiol Meas 27: 165-179, 2006.

57. Stolzmann P, Scheffel H, Leschka S, Schertler T, Frauenfelder T, Kaufmann PA, Marincek $\mathrm{B}$, and Alkadhi $\mathrm{H}$. Reference values for quantitative left ventricular and left atrial measurements in cardiac computed tomography. Eur Radiol 18: 1625-1634, 2008.

58. Streeter DD, Jr. Gross morphology and fiber geometry of the heart. In: Handbook of physiology, edited by Berne RM, Sperelakis N, and Geiger SR. Bethesda: American Physiological Society, 1979, p. 61-112.

59. Suga $H$, Hayashi $T$, and Shirahata $M$. Ventricular systolic pressure-volume area as predictor of cardiac oxygen consumption. Am J Physiol 240: H39-44, 1981.

60. Sun Y, Beshara M, Lucariello RJ, and Chiaramida SA. A comprehensive model for rightleft heart interaction under the influence of pericardium and baroreflex. Am J Physiol 272: H1499-1515, 1997.

61. Taher MF, Santamore WP, and Bogen DK. Ventricular interaction is described by three coupling coefficients. Am J Physiol 266: H228-234, 1994.

62. Tei C, Dujardin KS, Hodge DO, Bailey KR, McGoon MD, Tajik AJ, and Seward SB. Doppler echocardiographic index for assessment of global right ventricular function. J Am Soc Echocardiogr 9: 838-847, 1996.

63. Tei C, Dujardin KS, Hodge DO, Kyle RA, Tajik AJ, and Seward JB. Doppler index combining systolic and diastolic myocardial performance: Clinical value in cardiac amyloidosis. J Am Coll Cardiol 28: 658-664, 1996.

64. ter Keurs HE, Rijnsburger WH, van Heuningen R, and Nagelsmit MJ. Tension development and sarcomere length in rat cardiac trabeculae. Evidence of lengthdependent activation. Circ Res 46: 703-714, 1980.

65. Thistlethwaite PA, and Jamieson SW. Tricuspid valvular disease in the patient with chronic pulmonary thromboembolic disease. Curr Opin Cardiol 18: 111-116, 2003.

66. Usyk TP, Le Grice IJ, and McCulloch AD. Computational model of three-dimensional cardiac electromechanics. Comput Visual Sci 4: 249-257, 2002.

67. Watkins MW, and LeWinter MM. Physiologic role of the normal pericardium. Annu Rev Med 44: 171-180, 1993.

68. Weber KT, Janicki JS, Shroff S, and Fishman AP. Contractile mechanics and interaction of the right and left ventricles. Am J Cardiol 47: 686-695, 1981.

69. Wu Y, Cazorla O, Labeit D, Labeit S, and Granzier H. Changes in titin and collagen underlie diastolic stiffness diversity of cardiac muscle. J Mol Cell Cardiol 32: 2151-2162, 2000 . 
70. Yeo TC, Dujardin KS, Tei C, Mahoney DW, McGoon MD, and Seward JB. Value of a doppler-derived index combining systolic and diastolic time intervals in predicting outcome in primary pulmonary hypertension. Am J Cardiol 81: 1157-1161, 1998.

71. Yu CM, Sanderson JE, Chan S, Yeung L, Hung YT, and Woo KS. Right ventricular diastolic dysfunction in heart failure. Circulation 93: 1509-1514, 1996. 
TriSeg Model of Ventricular Mechanics and Hemodynamics 


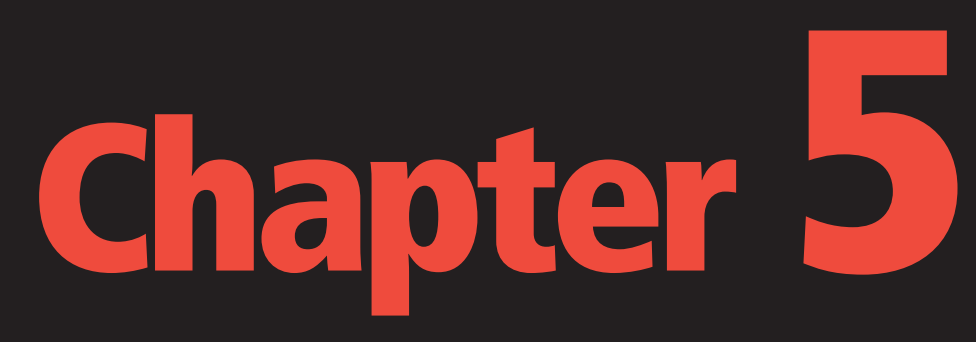

\title{
Right Ventricular Free Wall Pacing Improves Cardiac Pump Function in Severe Pulmonary Arterial Hypertension: a Computer Simulation Analysis
}

\author{
Joost Lumens 1,2 \\ Theo Arts 1 \\ Bernard Broers 3 \\ Karin A. Boomars 4 \\ Pieter van Paassen 5 \\ Frits W. Prinzen 1 \\ Tammo Delhaas ${ }^{1}$
}

Departments of ${ }^{1}$ Physiology and ${ }^{2}$ Biomedical Engineering, Cardiovascular Research Institute Maastricht (CARIM), Maastricht University; and Departments of ${ }^{3}$ Cardiology, ${ }^{4}$ Respiratory Diseases, and ${ }^{5}$ Internal Medicine, University Hospital Maastricht, Maastricht, The Netherlands.

Am J Physiol Heart Circ Physiol. 297: H2196-2205, 2009 


\section{ABstract}

In pulmonary arterial hypertension (PAH), duration of myofiber shortening is prolonged in the right ventricular (RV) free wall (RVfw) as compared to that in the interventricular septum and left ventricular (LV) free wall (LVfw). This interventricular mechanical asynchrony eventually leads to right heart failure. We investigated by computer simulation whether, in PAH, early RVfw pacing may improve interventricular mechanical synchrony and, hence, cardiac pump function. A mathematical model of the human heart and circulation was used to simulate LV and RV pump mechanics and myofiber mechanics. First, we simulated cardiovascular mechanics of a healthy adult at rest. Size and mass of heart and blood vessels were adapted so that mechanical tissue load was normalized. Second, compensated PAH was simulated by increasing mean pulmonary artery pressure (mPAP) to $32 \mathrm{mmHg}$ while applying load adaptation. Third, decompensated PAH was simulated by increasing mPAP further to 79 $\mathrm{mmHg}$ without further adaptation. Finally, early RVfw pacing was simulated in severely decompensated PAH. Time courses of circumferential strain in the ventricular walls as simulated were similar to the ones measured in healthy subjects (uniform strain patterns) and in PAH patients (prolonged RVfW shortening). When simulating pacing in decompensated PAH, RV pump function was best upon 40-ms RVfw preexcitation, as evidenced by maximal decrease of $\mathrm{RV}$ end-diastolic volume, reduced RVfw myofiber work, and most homogeneous distribution of workload over the ventricular walls. Thus, our simulations indicate that, in decompensated PAH, RVfw pacing may improve RV pump function and may homogenize workload over the ventricular walls. 


\section{INTRODUCTION}

In pulmonary arterial hypertension (PAH), progressive increase of pulmonary vascular resistance causes chronic elevation of right ventricular (RV) pressure. When left untreated, PAH leads to right heart failure and premature death. ${ }^{18,24}$ Several studies showed that morbidity and mortality of PAH patients are determined by the ability of the RV to maintain normal stroke volume and cardiac output. 6,23

In PAH, structural remodeling (hypertrophy and dilatation) is limited to the RV free wall (RVfw), ${ }^{5,19}$ suggesting an inhomogeneous distribution of myocardial tissue load. Therefore, local reduction of mechanical load of the RVfw may be another therapeutic option, in addition to conventional reduction of pulmonary vascular resistance. ${ }^{8}$

Marcus et al. ${ }^{17}$ demonstrated the presence of interventricular mechanical asynchrony in severe PAH patients, evidenced as prolonged duration of shortening and delayed peak shortening in the RVfw as compared to that in the LV free wall (LVfw) and the interventricular septum (Sept). RVfw shortening even continued after pulmonary valve closure and appeared related to leftward septal motion during LV isovolumic relaxation. As a potential treatment, early RVfw pacing was suggested to improve interventricular mechanical synchrony and thereby cardiac pump function. ${ }^{2,12,17}$ This seems logical, because during ventricular pacing, mechanical myofiber work is significantly decreased in early activated regions, whereas it is increased in late activated regions. ${ }^{21}$

In order to explore the idea of RV pacing in PAH, we used a mathematical mode ${ }^{16}$ to simulate ventricular mechanics and hemodynamics under normal and PAH conditions. The hypothesis was assessed that, in severe PAH, early pacing of the RVfw decreases mechanical myofiber load in the RVfw and thereby improves RV pump function.

\section{Methods}

\section{Model design}

For the present study, the TriSeg model, ${ }^{16}$ describing ventricular mechanics including mechanical interaction of the ventricular walls, was embodied in the CircAdapt model of the whole circulation. ${ }^{1}$

The TriSeg model of ventricular mechanics, previously described in more detail by Lumens et al. ${ }^{15,16}$ incorporates mechanical interaction of the LVfw, RVfw, and Sept resulting in realistic coupling characteristics of LV and RV pump mechanics. In short, three thick-walled spherical segments representing the 
ventricular walls join in a common junction so that they encapsulate the LV and RV cavity. Given LV and RV cavity volume, area and curvature of the LVfw and $\mathrm{RVfw}$ are calculated while assuming an initial estimate of Sept geometry. From wall geometry, representative myofiber strain is calculated for each wall. From myofiber strain, myofiber stress is determined using constitutive equations describing sarcomere mechanics. This latter empirical model incorporates sarcomere properties as derived from experiments on isolated cardiac muscle, ${ }^{7,26}$ i.e., velocity of sarcomere shortening increases with passive stretch, strength of activation increases with sarcomere length, and duration of activation increases with sarcomere length. Using myofiber stress and wall geometry, total radial and axial force components of tension acting on the junction are calculated. Then, septal geometry (initially estimated) is adjusted so that equilibrium of tensional force in the junction is satisfied. As a result, geometries of the three ventricular walls are known, together with myofiber stresses, wall tensions, and ventricular cavity volumes and pressures. Summarizing, the TriSeg model relates global LV and RV pump mechanics to local myofiber mechanics in the three ventricular walls. This relation is based on the principle of conservation of energy, meaning that summed ventricular pump work equals summed mechanical work as generated by the myofibers in the ventricular walls.

The CircAdapt model of the whole circulation ${ }^{1}$ creates the required physiological environment (circulatory boundary conditions) for the ventricles as represented by the TriSeg model. The CircAdapt model consists of a network of modules representing myocardial walls, valves, large blood vessels, and peripheral resistances. It allows beat-to-beat simulation of time-dependent ventricular mechanics and hemodynamics, e.g., volumes and pressures of the ventricular cavities, geometries and myofiber mechanics of the three ventricular walls, and flows through valves. An important feature of the CircAdapt model is that the number of independent parameters is reduced by incorporating adaptation of cardiac and vascular wall size and wall mass to mechanical load so that stresses and strains in the walls of heart and blood vessels are normalized to tissue-specific physiological standard levels. ${ }^{1,16}$

Pulmonary peripheral resistance was modeled as a non-linear resistive module connecting the compliant pulmonary arterial and venous blood vessels. Pulmonary flow was proportional to the squared pulmonary arteriovenous pressure drop, which was prescribed for each simulation in the present study. This relation is in agreement with the pressure-flow relationship as measured in the human and canine lung with normal as well as increased peripheral pulmonary resistance due to pulmonary vascular disease. ${ }^{11,14,28}$ 


\section{Simulations}

Cardiovascular mechanics and hemodynamics were simulated under normal ventricular loading conditions at rest (NORM), with compensated mild PAH (PAHComp), with moderately and severely decompensated PAH (PAHDecompMod and PAHDecompSev, respectively), and with additional pacing of the RVfw. Details of the methods used for simulation of ventricular mechanics and hemodynamics under normal and pulmonary hypertensive loading conditions have been published previously. ${ }^{16}$

\section{NORM simulation}

The NORM simulation represents the human circulation under normal ventricular loading conditions with regular resting values of heart rate (70 bpm), cardiac output ( $5 \mathrm{l} / \mathrm{min})$, and mean arterial blood pressure $(92 \mathrm{mmHg})$. The heart and large blood vessels were fully compensated by adaptation to mechanical load. ${ }^{16}$ The three ventricular walls were activated synchronously.

\section{PAH simulations}

The PAHComp simulation represents a state of mild compensated PAH. Mean pulmonary arteriovenous pressure drop was increased from $11 \mathrm{mmHg}(1.5 \mathrm{kPa})$ to $26 \mathrm{mmHg}(3.5 \mathrm{kPa})$, so that mean pulmonary artery pressure (mPAP) increased from $18 \mathrm{mmHg}$ (NORM) to $32 \mathrm{mmHg}$. Subsequently, size and mass of the heart and large blood vessels were fully compensated by adaptation to normalize mechanical load of the constituting tissues. This adaptation resulted in a $40 \%$ increase of RVfw mass. More details of the simulation protocol during adaptation have been published previously. ${ }^{16}$ The PAHDecompMod and PAHDecompSev simulations represent a moderate and a severe state of decompensated PAH, respectively. This pathological condition was achieved by further increase of mean pulmonary arteriovenous pressure drop to $49 \mathrm{mmHg}$ (6.5 kPa, PAHDecompMod) and $71 \mathrm{mmHg}(9.5 \mathrm{kPa}$, PAHDecompSev), so that mPAP increased to $55 \mathrm{mmHg}$ and $79 \mathrm{mmHg}$, respectively. In the PAHDecomp simulations, no further adaptation of ventricular and atrial walls to mechanical load was performed as compared to the PAHComp simulation, thus introducing partial RV decompensation. The three ventricular walls were activated synchronously.

\section{RVfw pacing simulations}

To study the acute effect of early RVfw pacing on ventricular mechanics in severely decompensated PAH, the RVfw in the PAHDecompSev simulation was activated 
20, 40, and 60 ms earlier than the LVfw and the Sept. No adaptation was applied. Finally, the same pacing protocol was applied to the NORM simulation as control measurement and to the PAHDecompMod simulation to investigate whether the effect of RVfw pacing in PAH depends on degree of decompensation.

\section{Data analysis}

In order to test whether the model realistically describes ventricular mechanics in $\mathrm{PAH}$, simulated time courses of circumferential strain in the ventricular walls were compared with clinical strain data obtained in healthy subjects and severe PAH patients by Marcus et al. ${ }^{17}$ In our simulations, for each ventricular wall, circumferential strain was quantified as the percent change in sarcomere length with respect to reference sarcomere length at moment of aortic valve opening.

For each simulation, LV and RV pressure waveforms were normalized to their maximum values and plotted as function of time. These normalized pressure curves were used to quantify timing difference $\left(\Delta t_{\text {dec }}\right)$ between LV and $\mathrm{RV}$ pressure decay at half maximum pressure. This timing difference was defined positive when RV pressure decay precedes LV pressure decay.

For each ventricular wall segment, mechanical myofiber load was represented by natural myofiber strain and Cauchy myofiber stress. The area of the obtained myofiber stress-strain loop represents stroke work density, defined as contractile myofiber work per unit of tissue volume (unit: $\mathrm{Pa}=\mathrm{Joule} / \mathrm{m}^{3}$ ) per beat.

\section{RESULTS}

Below, we first compare simulated and measured circumferential strain patterns under normal healthy and under severe PAH conditions (Figure 5.1). Next, we evaluate the effect of compensated and decompensated PAH on cardiac pump function in Figures 5.2, 5.3, and 5.4. Finally, we evaluate the effect of RVfw pacing in severely decompensated PAH on cardiac pump function in Figures 5.5, 5.6, and 5.7.

\section{NORM and PAH simulations}

In the NORM simulation as well as in the healthy subject, time courses of circumferential strain showed synchronous shortening of the ventricular walls during ejection, synchronous lengthening during filling, and similar values of peak circumferential shortening in the three walls (Figure 5.1, left panels). Also under severely decompensated PAH conditions, simulated and measured circumferential strain patterns were similar (Figure 5.1, right panels), with RVfw 
peak shortening being delayed with respect to LVfw and Sept peak shortening and with prolonged RVfw shortening that continues after aortic and pulmonary valve closure. During LV isovolumic relaxation, leftward bulging of the interventricular septum was associated with lengthening of myofibers in the

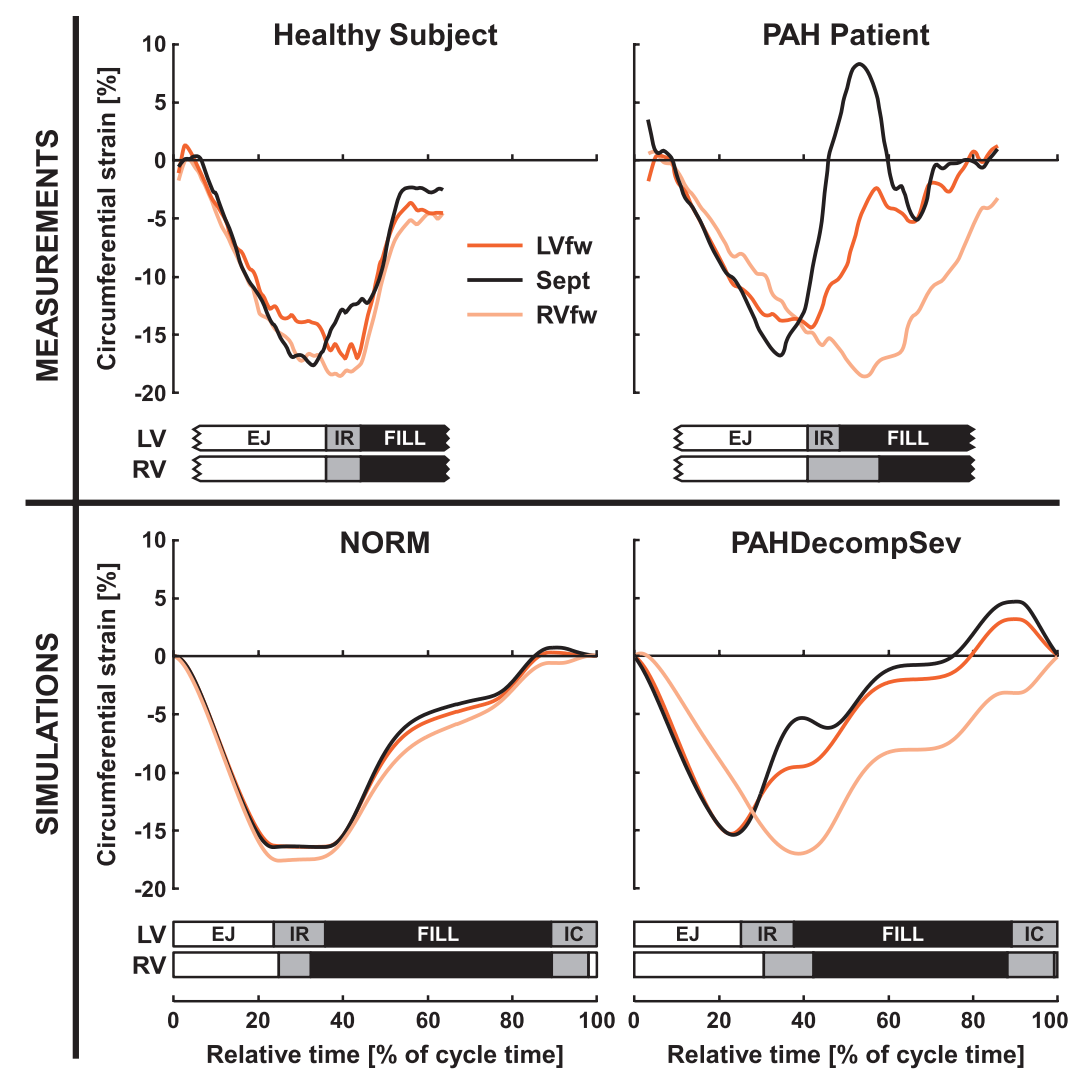

Figure 5.1. Time courses of circumferential strain in a healthy subject and a pulmonary arterial hypertension (PAH) patient: measurements (top row) [Adapted from Marcus et al. ${ }^{17}$ ] and simulations (bottom row). Both, measurements and simulations show uniform strain curves under normal loading conditions and interventricular mechanical asynchrony with severe PAH, i.e., prolonged duration of RVfw shortening, delayed RVfw peak shortening, and lengthening of the Sept and LVfw during left ventricular isovolumic relaxation. Timing of left ventricular (LV) and right ventricular (RV) filling (FILL), isovolumic contraction (IC), isovolumic relaxation (IR), and ejection (EJ) are indicated in the horizontal bar plots. LVfw, LV free wall; RVfw, RV free wall; and Sept, septal wall. 
Sept and LVfw. Maximal leftward septal bulging occurred at moment of RVfw peak shortening. Furthermore, simulated as well as measured RV isovolumic relaxation phase was prolonged and delayed in severe PAH as compared to that in the normal situation (horizontal bar plots in Figure 5.1).

In the upper panel row of Figure 5.2, time courses of ventricular pressures show that RV pressure increased with PAH. In the PAHDecompSev simulation, RV pressure even exceeded LV pressure during diastole and late systole. The time

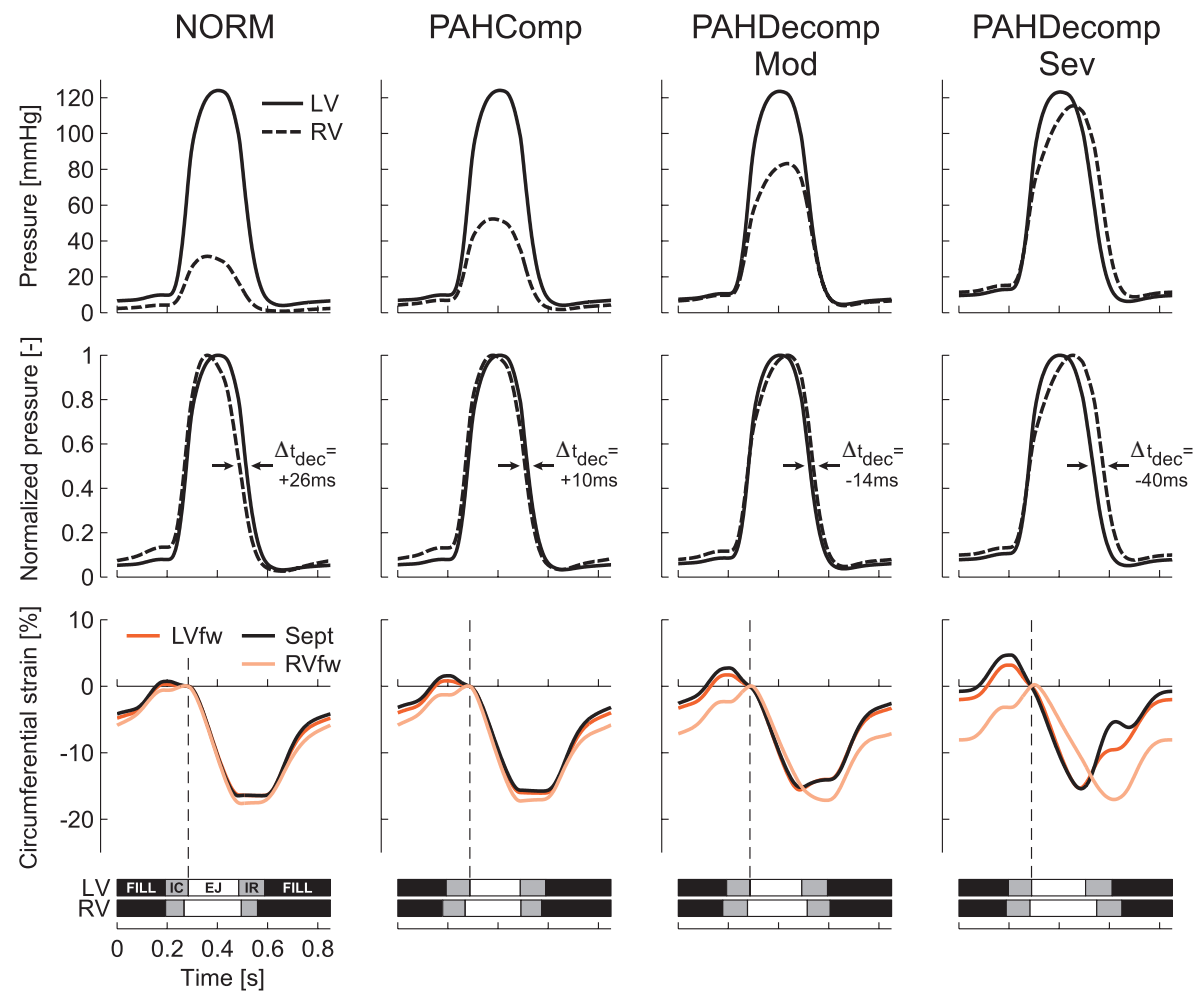

Figure 5.2. Time courses of simulated ventricular pressures and circumferential strains under normal (NORM), compensated pulmonary arterial hypertensive (PAHComp), and decompensated pulmonary arterial hypertensive ventricular loading conditions (PAHDecompMod, and PAHDecompSev). Upper panel row: left (LV) and right (RV) ventricular cavity pressures. Middle panel row: normalized LV and RV pressure curves. Lower panel row: circumferential strain in the LV free wall (LVfw), septum (Sept), and RV free wall (RVfw). Timing of LV and RV filling (FILL), isovolumic contraction (IC), isovolumic relaxation (IR), and ejection (EJ) are indicated in the horizontal bar plots. Vertical dashed lines indicate moment of aortic valve opening. 
courses of normalized LV and RV pressures show that LV and RV pressure rise synchronously in NORM, PAHComp, PAHDecompMod, and PAHDecompSev (middle panel row of Figure 5.2). PAH hardly affected timing of LV pressure rise and decay. RV pressure decay, on the other hand, delayed with increase of PAH. RV pressure decay preceded LV pressure decay in NORM and PAHComp, as reflected by positive values of $\Delta \mathrm{t}_{\mathrm{dec}}$, whereas decay of $\mathrm{LV}$ pressure preceded that of the RV in decompensated PAH, as reflected by negative values of $\Delta t_{\text {dec }}$. This delay of $R V$ pressure decay was associated with delayed $R V$ isovolumic relaxation.

In the NORM and PAHComp simulations, time courses of circumferential strains (lower panel row Figure 5.2) showed relatively synchronous and uniform strain patterns in the three ventricular walls. In each wall, peak shortening was reached at end-ejection. Peak shortening was $16 \%$ in the LVfw as well as in the Sept and $17 \%$ in the RVfw. However, with decompensation (PAHDecompMod and PAHDecompSev) circumferential strain patterns changed. During isovolumic contraction, the LVfw and Sept shortened, whereas the RVfw lengthened. Consequently, onset of RVfw shortening was delayed as compared to that of the LVfw and Sept. Peak RVfw shortening was also delayed till moment of mitral valve opening, whereas peak shortening of the LVfw and Sept still occurred at moment of aortic valve closure. The delay of RVfw peak shortening with respect to that of the LVfw was 106 and 136 ms for the PAHDecompMod and PAHDecompSev simulations, respectively. Duration of RVfw shortening increased from $212 \mathrm{~ms}$ in the NORM and PAHComp simulations to 294 and 318 $m s$ in the PAHDecompMod and PAHDecompSev simulations, respectively, whereas duration of LVfw and Sept shortening was similar (282 ms) in the NORM and the three PAH simulations.

As shown in Figure 5.3A, RV end-diastolic pressure increased from 4 $\mathrm{mmHg}$ in the NORM to $15 \mathrm{mmHg}$ in the PAHDecompSev simulation. LV enddiastolic pressure increased less, from $10 \mathrm{mmHg}$ in the NORM and PAHComp simulations to $11 \mathrm{mmHg}$ in PAHDecompMod and $13 \mathrm{mmHg}$ in PAHDecompSev. In the PAHComp simulation, RVfw mass and end-diastolic thickness were increased by $38 \%$ and $50 \%$, respectively, whereas LV and RV end-diastolic volumes were not changed with respect to the NORM simulation (Figure 5.3B). With decompensation, however, RV end-diastolic volume increased by $54 \%$ and LV end-diastolic volume decreased by $9 \%$ in PAHDecompSev. As compared to the

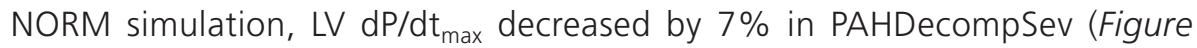
5.3C), while $R V d P / d t_{\max }$ increased by $175 \%$. 

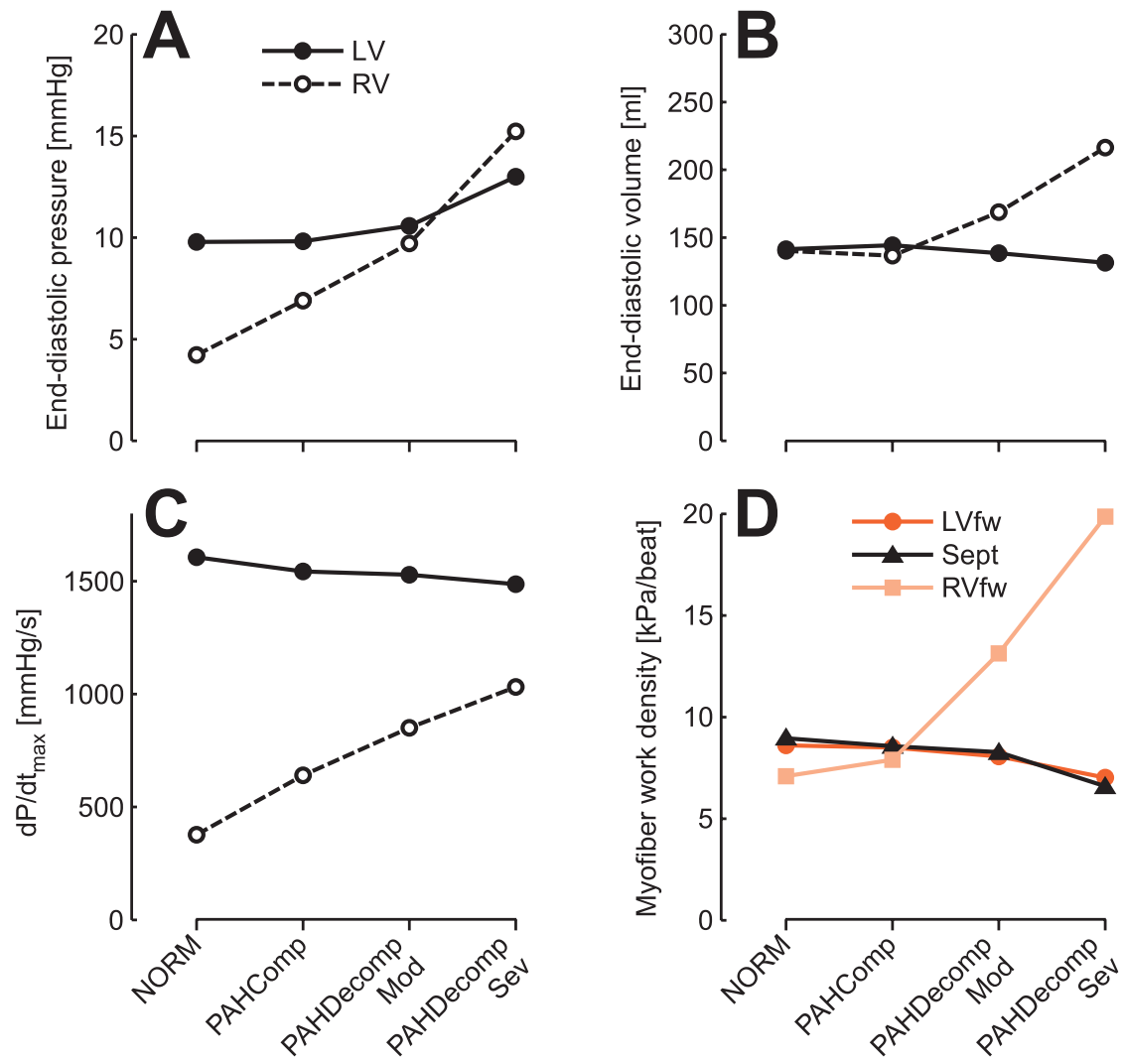

Figure 5.3. Simulated left (LV) and right (RV) ventricular end-diastolic pressures (A), end-diastolic volumes $(\mathbf{B})$, maximal rates of pressure rise $\mathrm{dP} / \mathrm{dt}_{\max }(\mathbf{C})$, and myofiber work densities (D) in the LV free wall (LVfw), septum (Sept), and RV free wall (RVfw) under normal (NORM), compensated pulmonary arterial hypertensive (PAHComp), and decompensated pulmonary arterial hyper-tensive ventricular loading conditions (PAHDecompMod and PAHDecompSev).

In the NORM and PAHComp simulations, mechanical load was distributed homogeneously over the ventricular walls as indicated by similarity of myofiber stress-strain relations (Figure 5.4). In PAHDecompMod and PAHDecompSev, however, mechanical load was inhomogeneously distributed over the ventricular walls. In the PAHDecompSev simulation, RVfw myofiber work density was increased by $182 \%$ with respect to that in the NORM simulation (Figures 5.3D and 5.4), whereas Sept and LVfw myofiber work densities were decreased by $27 \%$ and $18 \%$, respectively, predominantly due to myofiber lengthening during 
LV isovolumic relaxation (Figure 5.4). Peak RVfw myofiber stress was three times higher than in the NORM simulation. Furthermore, with decompensation, the RVfw myofiber stress-strain relation was shifted to the right, reflecting increase of mean RVfw sarcomere length from $1.9 \mu \mathrm{m}$ in the NORM and PAHComp simulations to $2.0 \mu \mathrm{m}$ and $2.2 \mu \mathrm{m}$ in the PAHDecompMod and PAHDecompSev simulations, respectively.
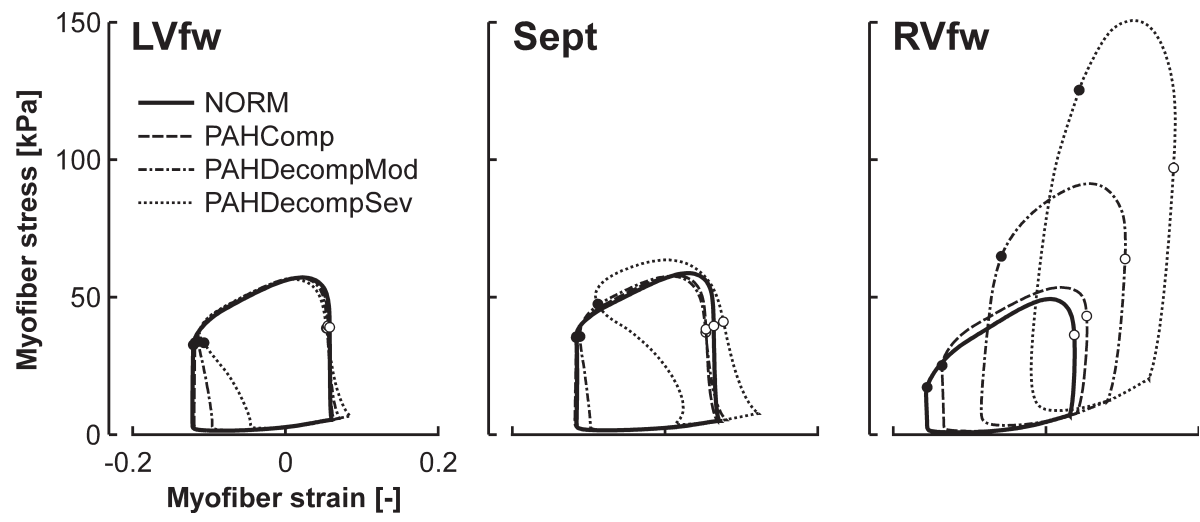

Figure 5.4. Simulated myofiber mechanics, in terms of natural myofiber strain and Cauchy myofiber stress, in the ventricular walls under normal (NORM), compensated pulmonary arterial hypertensive (PAHComp), and decompensated pulmonary arterial hypertensive ventricular loading conditions (PAHDecompMod and PAHDecompSev). Zero myofiber strain corresponds to a sarcomere length of $2.0 \mu \mathrm{m}$. Moments of aortic valve opening (open dots) and closure (closed dots) are indicated. LVfw, LV free wall; RVfw, RV free wall; and Sept, septal wall.

\section{RVfw pacing simulations}

As shown in the upper panel row of Figure 5.5, RVfw pacing did not affect the amplitude of ventricular pressures in severe $\mathrm{PAH}$. RV pressure rise and decay occurred earlier with RVfw pacing in PAH, while timing of LV pressure rise and decay did not change (middle panel row Figure 5.5). Timing difference $\Delta t_{\text {dec }}$ increased when activating the RVfw earlier than the LVfw and Sept, indicating resynchronization of LV and RV pressure decay. Early RVfw pacing decreased RVfw peak shortening, increased Sept and LVfw peak shortening, resynchronized LV and RV isovolumic relaxation phases, and reduced lengthening of the Sept and LVfw during LV isovolumic relaxation (lower panel row Figure 5.5). 


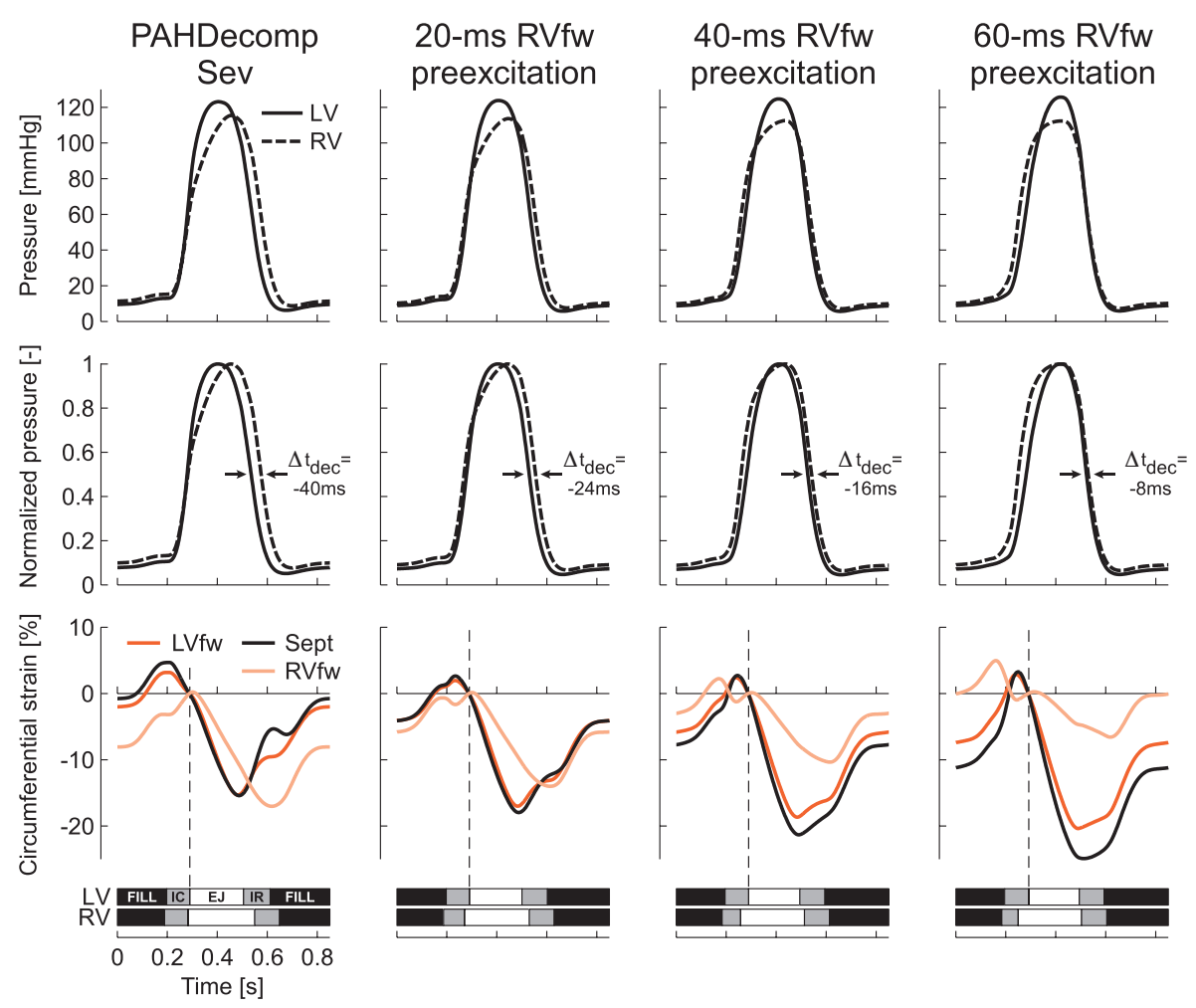

Figure 5.5. Time courses of simulated ventricular pressures and circumferential strains in severely decompensated pulmonary arterial hypertension with synchronous activation of the ventricular walls (PAHDecompSev) and with 20-, 40-, and 60-ms RV free wall preexcitation. Upper panel row: left (LV) and right (RV) ventricular cavity pressures. Middle panel row: normalized LV and RV pressure curves. Lower panel row: circumferential strain in the LV free wall (LVfw), septum (Sept), and RV free wall (RVfw). Timing of LV and RV filling (FILL), isovolumic contraction (IC), isovolumic relaxation (IR), and ejection (EJ) are indicated in the horizontal bar plots. Vertical dashed lines indicate moment of aortic valve opening.

RVfw pacing decreased RV end-diastolic pressure and volume (Figure 5.6). These parameters reached their minimal value $(-7 \%$ and $-6 \%$ as compared to PAHDecompSev, respectively) upon 40-ms RVfw preexcitation. In this latter simulation, LV end-diastolic pressure and volume were not changed. Furthermore, RV dP/dt $\max$ was increased (+13\%) and LV dP/dt $t_{\max }$ was decreased $(-25 \%)$ as compared to the PAHDecompSev simulation. 
With early RVfw pacing in severe PAH, RVfw myofiber work density decreased (Figure 5.6D). This decrease of RVfw myofiber work resulted from decrease of peak myofiber stress (up to $8 \%$ with $40-\mathrm{ms}$ RVfw preexcitation) and shortening of the RVfw (decrease of myofiber strain) between mitral valve closure and aortic valve opening (Figure 5.7). RVfw pacing also decreased myofiber lengthening in the Sept and LVfw after aortic valve closure, whereas shortening during ejection increased (Figure 5.7). As a consequence, RVfw pacing increased myofiber work density in the Sept and LVfw. Myofiber work was distributed most homogeneously over the ventricular walls upon 40-ms RVfw preexcitation (Figure 5.6D).
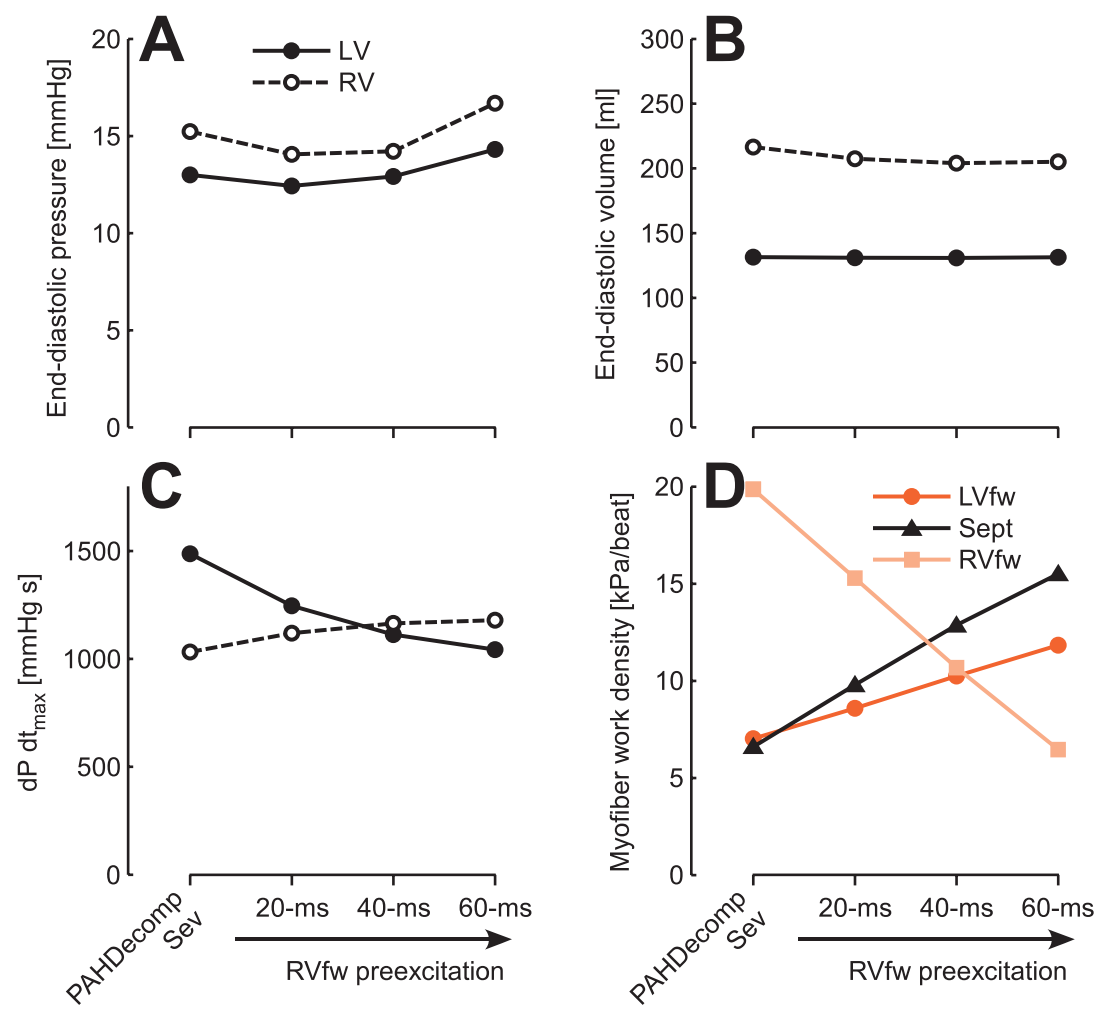

Figure 5.6. Simulated left (LV) and right (RV) ventricular end-diastolic pressures (A), end-diastolic volumes (B), maximal rates of pressure rise $\mathrm{dP} / \mathrm{dt}_{\max }(\mathbf{C})$, and myofiber work densities (D) in the LV free wall (LVfw), septum (Sept), and RV free wall (RVfw) in severely decompensated pulmonary arterial hypertension with synchronous activation of the ventricular walls (PAHDecompSev) and with 20-, 40-, and 60-ms RVfw preexcitation. 


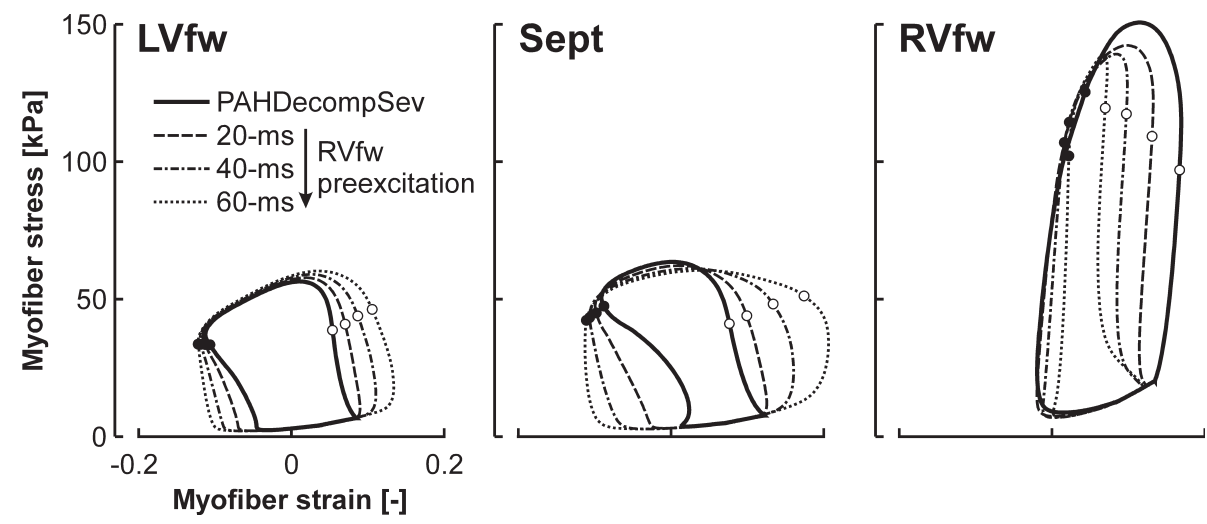

Figure 5.7. Simulated myofiber mechanics, in terms of natural myofiber strain and Cauchy myofiber stress, in the LV free wall (LVfw), septum (Sept), and RV free wall (RVfw) in severely decompensated pulmonary arterial hypertension with synchronous ventricular activation (PAHDecompSev) and with 20-, 40-, and 60-ms RVfw preexcitation. Zero myofiber strain corresponds to a sarcomere length of $2.0 \mu \mathrm{m}$. Moments of aortic valve opening (open dots) and closure (closed dots) are indicated.

The model predicted detrimental effects of RVfw preexcitation on ventricular pump function and myofiber mechanics under normal ventricular loading conditions (NORM simulation; Figure 5.8): LV and RV end-diastolic

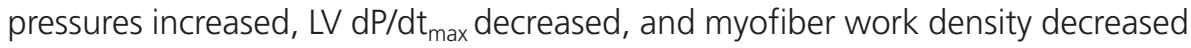
in the RVfw and Sept, whereas it increased in the LVfw. 

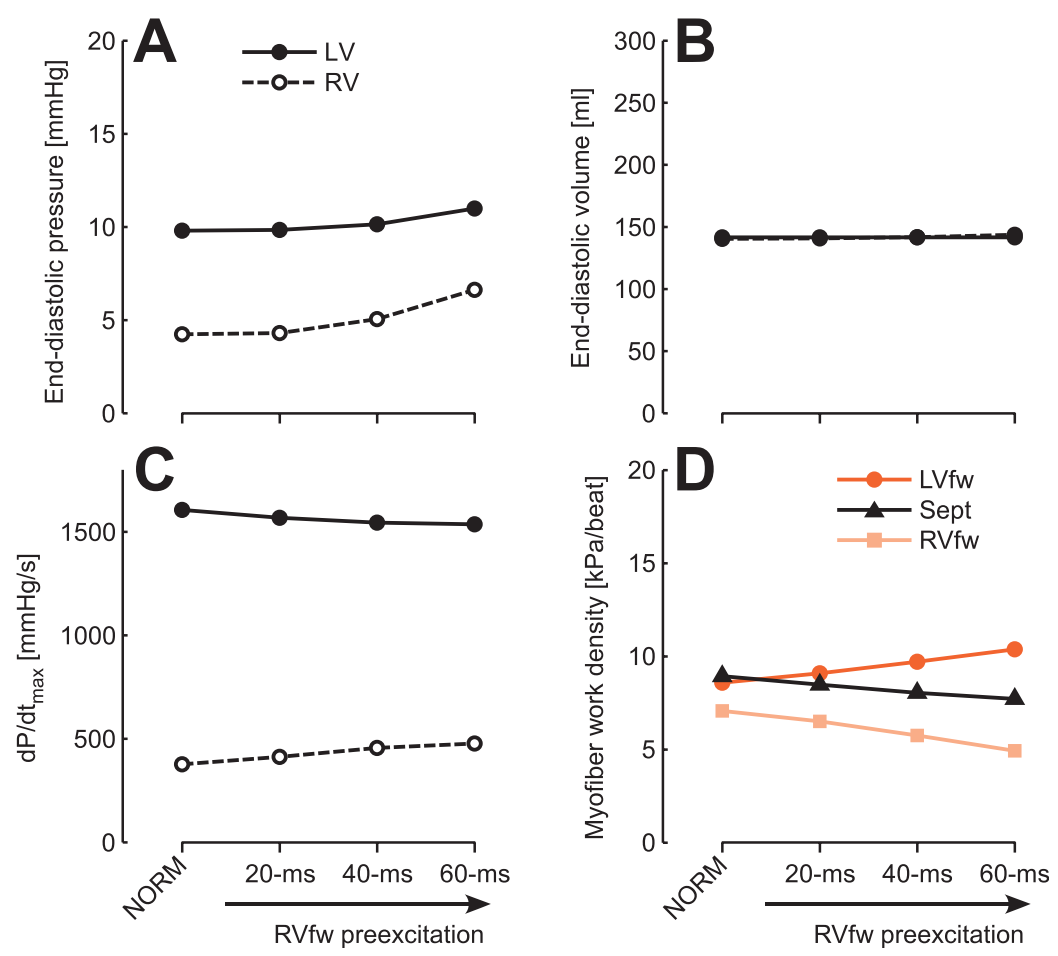

Figure 5.8. Simulated left (LV) and right (RV) ventricular end-diastolic pressures (A), end-diastolic volumes (B), maximal rates of pressure rise $\mathrm{dP} / \mathrm{dt}_{\max }(\mathbf{C})$, and myofiber work densities (D) in the LV free wall (LVfw), septum (Sept), and RV free wall (RVfw) under normal ventricular loading conditions with synchronous activation of the ventricular walls (NORM) and with 20-, 40-, and 60ms RVfw preexcitation.

Similarly as observed in severely decompensated PAH (Figure 5.6), RVfw preexcitation resulted in redistribution of myofiber work over the ventricular walls in moderately decompensated PAH (PAHDecompMod simulation; Figure 5.9). Under these moderate PAH conditions, myofiber work was distributed most homogeneously over the ventricular walls upon 20-ms RVfw preexcitation. This redistribution, however, was not associated with decrease of end-diastolic ventricular pressures or volumes. These simulation results show that, in our model, the beneficial effect of RVfw pacing on cardiac pump function in decompensated PAH increases with disease severity. 

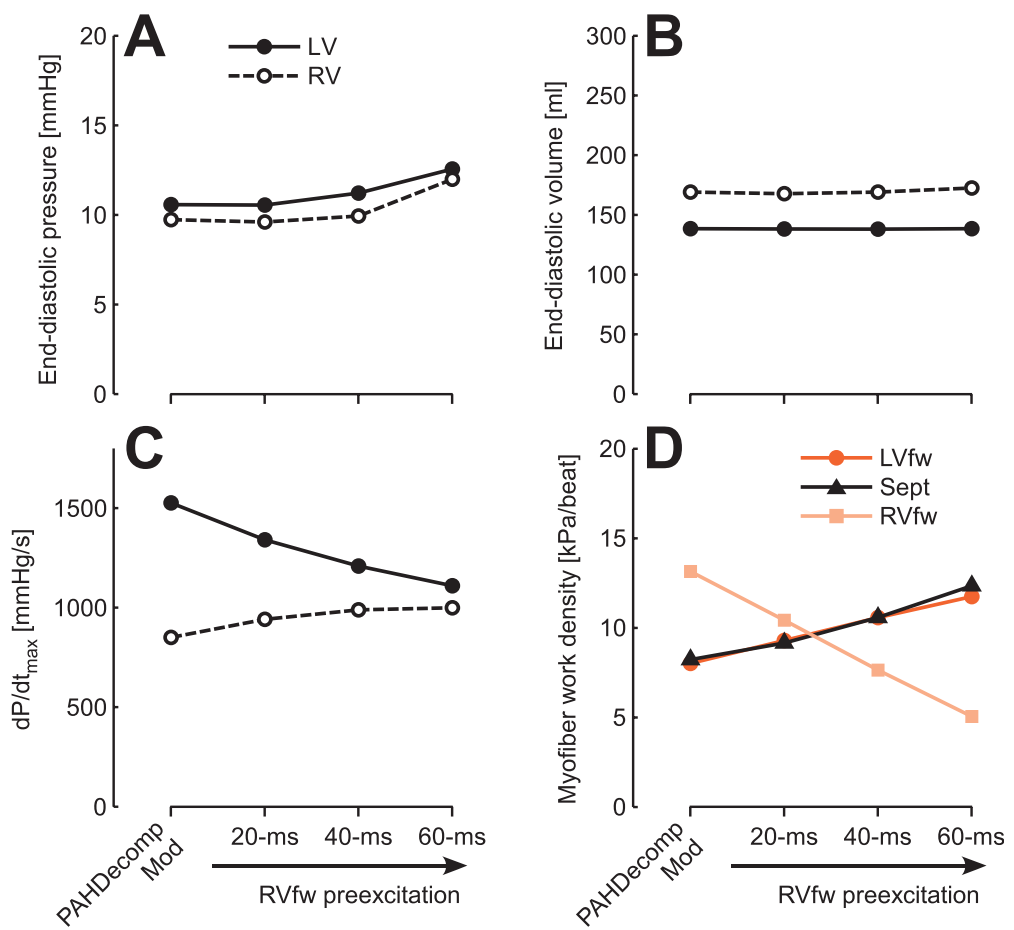

Figure 5.9. Simulated left (LV) and right (RV) ventricular end-diastolic pressures (A), end-diastolic volumes (B), maximal rates of pressure rise $\mathrm{dP} / \mathrm{dt}_{\max }(\mathbf{C})$, and myofiber work densities (D) in the LV free wall (LVfw), septum (Sept), and RV free wall (RVfw) in moderately decompensated pulmonary arterial hypertension with synchronous activation of the ventricular walls (PAHDecompMod) and with 20-, 40-, and 60-ms RVfw preexcitation.

\section{Discussion}

In the present study, a mathematical model describing mechanics of ventricular interaction was used to predict the effect of RVfw pacing on ventricular pump mechanics and hemodynamics in severe PAH. With severely decompensated $\mathrm{PAH}$, the model predicted an inhomogeneous distribution of myofiber load over the ventricular walls, expressed by three times higher myofiber work in the RVfw than in the Sept and LVfw. Interestingly, the model predicted that early RVfw pacing decreases the exorbitantly high myofiber work in the RVfw and increases myofiber work in the LVfw and Sept, while global LV and RV pump work remain the same. Apparently, the mechanical interaction of the ventricular walls 
allowed local redistribution of myofiber work over the ventricular walls. In our simulations, an optimal moment of RVfw preexcitation was found resulting in homogeneous distribution of myofiber work over the ventricular walls and moderate improvement of RV pump function.

\section{Comparison of measured and simulated ventricular geometry and wall mechanics in compensated and decompensated PAH}

Similarly as observed in experimental animals with mild chronic RV pressure overload, ${ }^{19,20}$ the RVfw was hypertrophied in the PAHComp simulation, while resting LV and RV end-diastolic volumes were not changed. Increasing pulmonary resistance without further cardiac load adaptation (PAHDecompMod and PAHDecompSev simulations), however, resulted in RV dilatation, decrease of LV volume, and prolonged as well as delayed RV isovolumic relaxation. All these changes are in agreement with measurements as obtained in patients with severe PAH. ${ }^{9,25}$ The simulated circumferential strain patterns in the three ventricular walls were in close agreement with measurements in healthy subjects and PAH patients. ${ }^{17}$ Measurements in and simulations of severe PAH showed prolonged RVfw shortening associated with lengthening of the Sept and LVfw during LV isovolumic relaxation. All these similarities between simulations and measurements show that our model enables realistic simulation of ventricular mechanics under normal as well as pulmonary arterial hypertensive ventricular loading conditions. Despite these qualitative similarities, however, lengthening of the Sept and LVfw during LV isovolumic relaxation appeared underestimated in the PAHDecompSev simulation as compared to the lengthening measured in the specific PAH patient of Figure 5.1. Apparently, the interventricular mechanical asynchrony as measured in the specific PAH patient cannot be entirely explained by normal sarcomere physiology as simulated by our sarcomere model. ${ }^{16}$ In the pathological PAH situation, the effect of decompensation on local myofiber mechanics may be aggravated. This is in agreement with the fact that in myocardial cells isolated from decompensated failing hearts action potential duration is prolonged as compared to that in normal cells. ${ }^{27}$

In patients with severe PAH, LV end-diastolic volume is significantly smaller $(\geq 25 \%)$ than in healthy controls. ${ }^{9,17}$ In our PAHDecompSev simulation, however, we observed a smaller decrease (9\%) (Figure 5.3B). This discrepancy most likely resulted from the assumption of constant cardiac output for all simulations. This was assumed in order to study the isolated effect of increased pulmonary peripheral resistance on ventricular mechanics and hemodynamics. In a clinical 
study comparing healthy controls with severe PAH patients, cardiac output was significantly smaller in PAH patients $(\geq 25 \%) .{ }^{9}$ If we would assume a similar reduction of cardiac output in our simulations, a similar change of LV enddiastolic volume is expected.

\section{Comparison of measured and simulated effects of pacing in decompensated PAH}

The beneficial effect of early RVfw pacing in chronically RV overloaded hearts seems to be supported by experimental data published recently by Handoko et al. ${ }^{10}$ In this study, the effect of RVfw pacing was investigated in a Langendorff set up of hearts isolated from rats with chronic pulmonary hypertension and right heart failure. Similarly as observed in our simulations, with RVfw preexcitation, an optimal pacing setting was found resulting 1) in maximal improvement of RV systolic function ( $8.5 \%$ increase of $\left.\mathrm{RV} \mathrm{dP} / \mathrm{dt}_{\max }\right), 2$ ) in decrease of timing difference of LV and RV pressure decay, and 3) in resynchronization of LV and RV peak pressure. Furthermore, in this study, RVfW pacing did not detrimentally affect LV pump function or coronary perfusion. Thus, the results of our simulation study and of the experimental study by Handoko et al. mutually support and complement each other.

In an open-chest pig model of acute RV pressure overload and complete AV-block, Quinn et al. ${ }^{22}$ also showed a beneficial effect of RVfw pacing on cardiac performance. In this study, early activation of the RVfw with respect to the LVfw resulted in increase of cardiac output, decrease of LV dP/dt $t_{\max }$, and increase of $\mathrm{RV} \mathrm{dP} / \mathrm{dt}_{\max }$. In our simulations, the largest decrease of RV enddiastolic pressure and volume was observed with 40-ms RVfw preexcitation (Figure 5.6). Similarly, in the experiments by Quinn et al., maximal increases of cardiac output and RV dP/dt $t_{\max }$ occurred when the RVfw was 37 ms earlier activated than the LVfw. Simulations as well as measurements showed that, during this optimal pacing setting, LV and RV pressure waveforms were resynchronized.

The simulations of RVfw pacing in a normal heart (Figure 5.8) are in agreement with experimental findings on RV pacing as well as left bundle branch block, indicating the combination of impairment of LV pump function, decrease of Sept and, to a greater extent, of RVfw workload, increase of LVfw workload, and moderate increase of RV pump function (RV dP/dt $\left.t_{\max }\right){ }^{13,21}$ 


\section{Potential mechanism behind interventricular mechanical asynchrony in PAH}

It is important to realize that in the PAHDecomp simulations all three ventricular walls were activated simultaneously, thus simulating a decompensated PAH condition without conduction delay. The mechanical asynchrony, especially the prolonged RVfw shortening observed in the PAHDecomp simulations, is explained by the basic property of our model ${ }^{16}$ that duration of mechanical myofiber activation increases with sarcomere length. This latter property is well known from experiments on isolated cardiac muscle. ${ }^{26}$ Our simulation results suggest that interventricular mechanical asynchrony in PAH is a direct consequence of stretching the RVfw (RV decompensation). Therefore, it seems safe to assume that the interventricular mechanical asynchrony as reported among patients with severe PAH by Marcus et al. also indicates RV decompensation. ${ }^{17} \mathrm{~A}$ spin-off of the present study may therefore be that detection of mechanical interventricular asynchrony could be used as an index of RV decompensation.

\section{Study limitations}

Obviously, conditions in a computer simulation may differ considerably from those in patients. In our model, each ventricular wall is lumped into a spherical wall segment containing a single contractile fiber describing representative passive and active sarcomere properties of the entire wall. ${ }^{16}$ Although this simplified setup allows inhomogeneity of material properties to some extent, i.e., between ventricular walls, it does not allow local inhomogeneities of material properties within a single wall. In our RVfw pacing simulations, the effect of activation spread away from the pacing site was not included. Mechanical activation time of the RVfw represented representative average activation time. Therefore, with this model we were not able to test the effect of pacing at different locations within the RVfw. Nevertheless, the qualitative and sometimes quantitative agreement between our model simulations and experimental as well as clinical observations indicate that the simplifications made in the simulations may not obscure first-order effects of RVfw pacing on ventricular mechanics and pump function in severe PAH. The present study only focuses on the acute hemodynamic effects of RVfw pacing. Long-term effects are not considered. However, in analogy with cardiac resynchronization in dyssynchronous ventricles, acute hemodynamic effects can be expected to be maintained over time and may even lead to further improvements by reverse remodeling. ${ }^{3}$ 
The effect of tricuspid regurgitation, which often exists in severe PAH and which leads to RV volume overload, is not included in the present simulations. RVfW pacing may aggravate tricuspid regurgitation by changing synchrony of RV papillary muscle activation but may also decrease it by reducing RV end-diastolic volume and thereby tricuspid annular dilatation.

Previous experimental studies showed that PAH leading to RV decompensation and heart failure is associated with structural remodeling of the RVfw myocardium (summarized in a review by Bogaard et al. ${ }^{4}$ ). Ischemia and interstitial fibrosis may locally change active and passive myocardial material properties in severe PAH. Although such changes were not included in our simulations of decompensated $\mathrm{PAH}$, we expect minor influence on the decreasing effect of early RVfw pacing on local mechanical work. Whether the translation of work homogenization to improvement of cardiac pump function, as observed in our simulations, is affected by such change of myocardial material properties is unknown. Nonetheless, Handoko et al. showed acute improvement of RV pump function with RVfw pacing in remodeled and failing hearts of rats with PAH. ${ }^{10}$

\section{Clinical implications}

The present study may provide a theoretical framework for a novel therapeutic strategy for severely decompensated PAH patients. Although decrease of pulmonary vascular resistance remains the primary therapeutic target in PAH patients, RVfw pacing as an additional treatment may contribute to clinical improvement of the patient by unloading the RVfw and thereby delaying development of RV failure. Furthermore, it might be useful as bridge to thoracic organ transplantation in these patients. Clearly, more experimental and clinical evidence is needed to prove that the predicted beneficial effect of RVfw pacing is clinically relevant for a PAH patient.

\section{CONCLUSIONS}

Our simulations of ventricular mechanics under pulmonary arterial hypertensive conditions show many similarities with data obtained in patients. Both, simulations and measurements show interventricular mechanical asynchrony in severely decompensated PAH, associated with prolonged duration of RVfw shortening and inhomogeneous distribution of myofiber load over the ventricular walls. Early RVfw pacing results in more synchronous LV and RV pressure decay, more homogeneous distribution of myofiber load over the 
ventricular walls, and slight improvement of RV pump function. Our simulations suggest that the potential of RVfw pacing as a novel therapeutic approach in PAH patients should be further investigated.

\section{GRANTS}

This work was financially supported by the Netherlands Heart Foundation Grant 2007 B203.

\section{Disclosures}

This work was financially supported by Actelion Pharmaceuticals Nederland B.V. (Woerden, The Netherlands). T. Delhaas receives research grants from Actelion Pharmaceuticals Nederland B.V. (Woerden, The Netherlands). F.W. Prinzen receives research grants from Medtronic Inc. (Minneapolis, MN), Boston Scientific Corp. (St. Paul, MN), and EBR Systems Inc. (Sunnyvale, CA). 
Chapter 5 


\section{REFERENCES}

1. Arts T, Delhaas T, Bovendeerd P, Verbeek X, and Prinzen FW. Adaptation to mechanical load determines shape and properties of heart and circulation: The circadapt model. Am J Physiol Heart Circ Physiol 288: H1943-1954, 2005.

2. Beyar R. Heart inefficiency in pulmonary hypertension: A double jeopardy. J Am Coll Cardiol 51: 758-759, 2008.

3. Bleeker GB, Schalij MJ, Nihoyannopoulos P, Steendijk P, Molhoek SG, van Erven L, Bootsma M, Holman ER, van der Wall EE, and Bax JJ. Left ventricular dyssynchrony predicts right ventricular remodeling after cardiac resynchronization therapy. J Am Coll Cardiol 46: 2264-2269, 2005.

4. Bogaard HJ, Abe K, Vonk Noordegraaf A, and Voelkel NF. The right ventricle under pressure: Cellular and molecular mechanisms of right-heart failure in pulmonary hypertension. Chest 135: 794-804, 2009.

5. Bouchard A, Higgins CB, Byrd BF, 3rd, Amparo EG, Osaki L, and Axelrod R. Magnetic resonance imaging in pulmonary arterial hypertension. Am J Cardiol 56: 938-942, 1985.

6. Chin KM, Kim NH, and Rubin LJ. The right ventricle in pulmonary hypertension. Coron Artery Dis 16: 13-18, 2005.

7. de Tombe PP, and ter Keurs HE. Force and velocity of sarcomere shortening in trabeculae from rat heart. Effects of temperature. Circ Res 66: 1239-1254, 1990.

8. Galie N, Torbicki A, Barst R, Dartevelle P, Haworth S, Higenbottam T, Olschewski H, Peacock A, Pietra G, Rubin LJ, Simonneau G, Priori SG, Garcia MA, Blanc JJ, Budaj A, Cowie M, Dean V, Deckers J, Burgos EF, Lekakis J, Lindahl B, Mazzotta G, McGregor K, Morais J, Oto A, Smiseth OA, Barbera JA, Gibbs S, Hoeper M, Humbert M, Naeije R, and Pepke-Zaba J. Guidelines on diagnosis and treatment of pulmonary arterial hypertension. The task force on diagnosis and treatment of pulmonary arterial hypertension of the european society of cardiology. Eur Heart J 25: 2243-2278, 2004.

9. Gan CT, Lankhaar JW, Marcus JT, Westerhof N, Marques KM, Bronzwaer JG, Boonstra A, Postmus PE, and Vonk-Noordegraaf A. Impaired left ventricular filling due to right-to-left ventricular interaction in patients with pulmonary arterial hypertension. Am J Physiol Heart Circ Physiol 290: H1528-1533, 2006.

10. Handoko ML, Lamberts RR, Redout EM, de Man FS, Boer C, Simonides WS, Paulus WJ, Westerhof N, Allaart CP, and Vonk-Noordegraaf A. Right ventricular pacing improves right heart function in experimental pulmonary arterial hypertension: A study in the isolated heart. Am J Physiol Heart Circ Physiol 297: H1752-1759, 2009.

11. Janicki JS, Weber KT, Likoff MJ, and Fishman AP. The pressure-flow response of the pulmonary circulation in patients with heart failure and pulmonary vascular disease. Circulation 72: 1270-1278, 1985. 


\section{Chapter 5}

12. Keogh AM, Mayer E, Benza RL, Corris P, Dartevelle PG, Frost AE, Kim NH, Lang IM, Pepke-Zaba J, and Sandoval J. Interventional and surgical modalities of treatment in pulmonary hypertension. J Am Coll Cardiol 54: S67-77, 2009.

13. Kerckhoffs RC, Lumens J, Vernooy K, Omens JH, Mulligan LJ, Delhaas T, Arts T, McCulloch AD, and Prinzen FW. Cardiac resynchronization: Insight from experimental and computational models. Prog Biophys Mol Biol 97: 543-561, 2008.

14. Kondo M, Washizu M, Matsukura Y, Washizu T, Miyasaka K, and Takata M. Pressureflow relationship and longitudinal distribution of pulmonary vascular resistance in heartworm-infected dogs. J Vet Med Sci 65: 965-970, 2003.

15. Lumens J, Delhaas T, Kirn B, and Arts T. Modeling ventricular interaction: A multiscale approach from sarcomere mechanics to cardiovascular system hemodynamics. Pac Symp Biocomput 378-389, 2008.

16. Lumens J, Delhaas T, Kirn B, and Arts T. Three-wall segment (TriSeg) model describing mechanics and hemodynamics of ventricular interaction. Ann Biomed Eng 37: 22342255, 2009.

17. Marcus JT, Gan CT, Zwanenburg JJ, Boonstra A, Allaart CP, Gotte MJ, and VonkNoordegraaf A. Interventricular mechanical asynchrony in pulmonary arterial hypertension: Left-to-right delay in peak shortening is related to right ventricular overload and left ventricular underfilling. J Am Coll Cardiol 51: 750-757, 2008.

18. McLaughlin VV. Survival in patients with pulmonary arterial hypertension treated with first-line bosentan. Eur J Clin Invest 36 Suppl 3: 10-15, 2006.

19. Minami S, Onodera T, Okazaki F, Miyazaki H, Ohsawa S, and Mochizuki S. Myocyte morphological characteristics differ between the phases of pulmonary hypertensioninduced ventricular hypertrophy and failure. Int Heart J 47: 629-637, 2006.

20. Olivetti G, Ricci R, Lagrasta C, Maniga E, Sonnenblick EH, and Anversa P. Cellular basis of wall remodeling in long-term pressure overload-induced right ventricular hypertrophy in rats. Circ Res 63: 648-657, 1988.

21. Prinzen FW, Hunter WC, Wyman BT, and McVeigh ER. Mapping of regional myocardial strain and work during ventricular pacing: Experimental study using magnetic resonance imaging tagging. J Am Coll Cardiol 33: 1735-1742, 1999.

22. Quinn TA, Berberian G, Cabreriza SE, Maskin LJ, Weinberg AD, Holmes JW, and Spotnitz HM. Effects of sequential biventricular pacing during acute right ventricular pressure overload. Am J Physiol Heart Circ Physiol 291: H2380-2387, 2006.

23. Sandoval J, Bauerle O, Palomar A, Gomez A, Martinez-Guerra ML, Beltran M, and Guerrero ML. Survival in primary pulmonary hypertension. Validation of a prognostic equation. Circulation 89: 1733-1744, 1994. 
24. Simonneau G, Galie N, Rubin L, Langleben D, Seeger W, Domenighetti G, Gibbs S, Lebrec D, Speich R, Beghetti M, Rich S, and Fishman A. Clinical classification of pulmonary hypertension. J Am Coll Cardiol 43: 5S-12S, 2004.

25. Stojnic BB, Brecker SJ, Xiao HB, Helmy SM, Mbaissouroum M, and Gibson DG. Left ventricular filling characteristics in pulmonary hypertension: A new mode of ventricular interaction. Br Heart J 68: 16-20, 1992.

26. ter Keurs HE, Rijnsburger WH, van Heuningen R, and Nagelsmit MJ. Tension development and sarcomere length in rat cardiac trabeculae. Evidence of lengthdependent activation. Circ Res 46: 703-714, 1980.

27. Tomaselli GF, and Marban E. Electrophysiological remodeling in hypertrophy and heart failure. Cardiovasc Res 42: 270-283, 1999.

28. West JB, Dollery CT, and Naimark A. Distribution of blood flow in isolated lung; relation to vascular and alveolar pressures. J Appl Physiol 19: 713-724, 1964. 



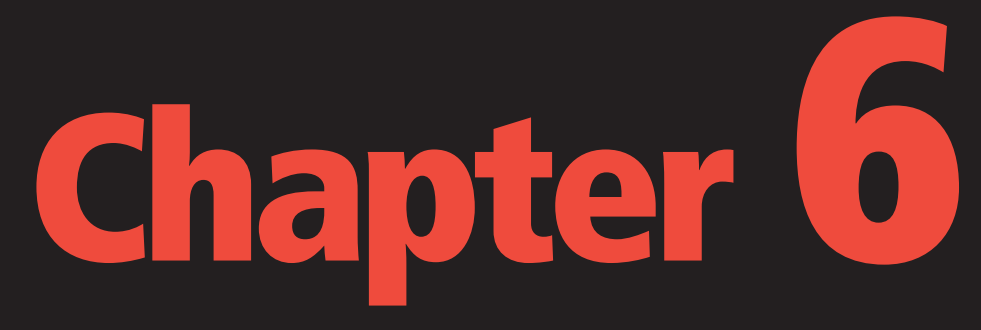

Left Ventricular Underfilling and Not Septal Bulging Dominates Abnormal Left Ventricular Hemodynamics in Chronic Thromboembolic Pulmonary Hypertension

\author{
Joost Lumens ${ }^{1,2}$ \\ Daniel G. Blanchard ${ }^{3}$ \\ Theo Arts 2 \\ Ehtisham Mahmud \\ Tammo Delhaas ${ }^{1,2}$
}

Departments of ${ }^{1}$ Physiology and ${ }^{2}$ Biomedical Engineering, Cardiovascular

Research Institute Maastricht (CARIM), Maastricht University, Maastricht, The Netherlands. Division of ${ }^{3}$ Cardiology, University of California, San Diego School of Medicine and UCSD Medical Center, San Diego, California.

Submitted 


\section{ABstract}

Chronic thromboembolic pulmonary hypertension (CTEPH) is associated with abnormal left ventricular (LV) filling hemodynamics (mitral E/A<1). Pulmonary thromboendarterectomy (PTE) acutely reduces pulmonary vascular resistance resulting in increase of mitral E/A. Abolishment of leftward septal bulging and increase in right ventricular (RV) systolic output are thought to be responsible for increase of mitral E/A. In this study, we quantified the separate effects of leftward septal bulging and of RV systolic output on LV hemodynamics. In 39 CTEPH patients who underwent PTE, transmitral flow velocities and RV hemodynamic data were obtained pre- and postoperatively. A mathematical model describing mechanics of ventricular interaction was fitted to preoperative average values of cardiac output (CO; $4.4 \mathrm{l} / \mathrm{min})$, mean pulmonary artery pressure (mPAP; $50 \mathrm{mmHg}$ ), mitral E/A (0.74), and mean left atrial pressure (mLAP; $9.8 \mathrm{mmHg}$ ). Starting from this preoperative reference state with leftward septal bulging, PTE was simulated by changing MPAP and CO to average postoperative values $(28 \mathrm{mmHg}$ and $5.7 \mathrm{l} / \mathrm{min}$, respectively). Simulated and postoperatively measured data on E/A (1.27 vs 1.48), mLAP (12.6 vs 11.5 $\mathrm{mmHg}$ ), and septal curvature (both rightward) were consistent. Simulating exclusive decrease of mPAP, mitral E/A increased $26 \%$ due to facilitated passive LV filling at $16 \%$ lower $\mathrm{mLAP}$, and septal curvature became rightward. Simulating exclusive increase of CO, mitral E/A increased 53\%, mLAP increased $62 \%$, whereas leftward septal bulging persisted. Thus, our simulations suggest that increase of mitral E/A with PTE is for two-third caused by increase of RV systolic output and for one-third by abolishment of leftward septal bulging. 


\section{INTRODUCTION}

In patients with chronic thromboembolic pulmonary hypertension (CTEPH), pulmonary vascular resistance (PVR) is chronically increased due to resistive lesions in the pulmonary vascular bed. The chronic increase of right ventricular (RV) afterload in these patients is associated with an abnormal left ventricular (LV) filling pattern, 7, 12, 27, 31 i.e., peak blood flow velocity of the early passive mitral filling wave (E-velocity) is smaller than that of the atrial contractioninduced late active filling wave (A-velocity). Successful treatment with surgical pulmonary thromboendarterectomy (PTE) leads to acute reduction of PVR associated with decrease of mean pulmonary artery pressure (MPAP) and with increase of cardiac output (CO). ${ }^{7,27}$ Consequently, PTE results in acute improvement of cardiac pump function. ${ }^{14,31,43}$ Furthermore, mitral E-velocity increases so that mitral $E / A$ changes from abnormal value $(E / A<1)$ to normal physiological value $(E / A>1)$.

Generally, two different mechanisms have been hypothesized to be responsible for the acute change of the LV filling pattern in CTEPH patients undergoing PTE. The first mechanism comprises the effect of PTE on LV filling via the pulmonary circulation, i.e., reduction of RV afterload leads to increase of RV systolic output and, thus, to increase of LV preload. The second mechanism comprises the effect directly transmitted by the interventricular septum, i.e., reduction of $\mathrm{RV}$ afterload is associated with re-establishment of the normal negative transseptal pressure gradient (LV pressure > RV pressure) and, thus, with abolishment of leftward septal bulging. This acute change of septal geometry is assumed to facilitate LV filling.

In the present study, we aimed to quantify the separate contributions of both mechanisms to the change of LV filling hemodynamics in CTEPH patients undergoing PTE.

\section{Materials And Methods}

In this study, clinical measurements, as obtained in a group of CTEPH patients undergoing PTE, are combined with a computational modeling approach that allows quantitative discrimination between the effect of increased RV systolic output and of decreased leftward septal bulging on LV filling hemodynamics in these patients. 


\section{Patient data}

Patient characteristics and a full description of data acquisition have been published previously. ${ }^{27}$ For the present study, relevant details of the patient population, of the echocardiographic and catheterization measurements, and of the statistical analysis, are summarized below.

\section{Patient population}

The patient group consisted of 39 consecutive patients (24 men and 15 women, mean age $55 \pm 11$ years) with surgically accessible CTEPH who underwent successful PTE. All patients were classified as New York Heart Association class III and IV.

\section{Echocardiography}

All patients underwent a standard echocardiographic examination $10 \pm 7$ days $($ mean \pm SD) before PTE. Pulsed-wave Doppler imaging of transmitral flow velocities was performed to quantify $E$ and $A$ mitral flow velocities. Heart rate was determined from the electrocardiogram during the Doppler echocardiographic measurements. This echocardiography protocol was repeated $10 \pm 6$ days after PTE.

\section{Right heart catheterization}

All patients underwent right heart catheterization within $48 \mathrm{~h}$ of preoperative echocardiography. A 7.5F Swan Ganz catheter was used for invasive measurement of mPAP and mean pulmonary capillary wedge pressure. Cardiac output was obtained by the thermodilution method. Mean arterial blood pressure was measured noninvasively. This catheterization protocol was repeated postoperatively within $72 \mathrm{~h}$ of the postoperative echocardiogram.

\section{Statistics}

Echocardiographic and hemodynamic patient data are expressed as mean \pm SD and are summarized in Table 6.1. A two-tailed Student t-test for paired populations was used to compare pre- and postoperative measurements of echocardiographic and hemodynamic parameters. A $p$-value $<0.05$ was considered statistically significant. 
Table 6.1. Catheterization and echocardiographic data obtained in 39 CTEPH patients before and after PTE.

\begin{tabular}{lccc} 
& Before PTE & After PTE & p-value \\
\hline Catheterization data & & & \\
\hline CO (I/min) & $4.4 \pm 1.1$ & $5.7 \pm 0.9$ & $<0.0001$ \\
mPAP (mmHg) & $50 \pm 11$ & $28 \pm 9$ & $<0.0001$ \\
PCWP (mmHg) & $9.8 \pm 5.0$ & $11.5 \pm 4.5$ & 0.04 \\
MAP (mmHg) & $93 \pm 6$ & $95 \pm 7$ & NS \\
\hline Echocardiographic data & & & \\
\hline E (cm/s) & $52.5 \pm 20.5$ & $82.5 \pm 18.5$ & $<0.0001$ \\
A (cm/s) & $68.0 \pm 17.9$ & $60.1 \pm 17.1$ & NS \\
E/A (-) & $0.74 \pm 0.22$ & $1.48 \pm 0.69$ & $<0.0001$ \\
HR (bpm) & $80 \pm 16$ & $81 \pm 15$ & NS \\
\hline
\end{tabular}

These patient data have been previously published by Mahmud et al. ${ }^{22}$. NS indicates that the parameter is not significantly different after PTE.

$A=$ peak flow velocity of late mitral filling wave; $C O=$ cardiac output; $C T E P H=$ chronic thromboembolic pulmonary hypertension; $E$ = peak flow velocity of early mitral filling wave; $E / A=$ mitral $\mathrm{E} / \mathrm{A}$ ratio; $\mathrm{HR}=$ heart rate; $\mathrm{MAP}=$ mean arterial blood pressure; $\mathrm{mPAP}=$ mean pulmonary artery pressure; $\mathrm{PCWP}=$ pulmonary capillary wedge pressure; $\mathrm{PTE}=$ pulmonary thromboendarterectomy.

\section{Computer simulations}

\section{Model design}

For the present study, the TriSeg model ${ }^{25}$ of ventricular mechanics was embodied in the CircAdapt model describing mechanics of the whole human circulation. ${ }^{1}$

The TriSeg model of ventricular mechanics, previously described in more detail by Lumens et al., ${ }^{24,25}$ incorporates mechanical interaction of the LV free wall, the RV free wall, and the interventricular septum resulting in realistic coupling characteristics of LV and RV pump mechanics. In short, three thickwalled spherical segments representing the ventricular walls join in a common junction so that they encapsulate the LV and RV cavity. Given LV and RV cavity volume, area and curvature of the LV and RV free wall are calculated while assuming an initial estimate of septal geometry. From wall geometry, representative myofiber strain is calculated for each wall. From myofiber strain, 
myofiber stress is determined using constitutive equations describing sarcomere mechanics. The latter empirical model incorporates sarcomere properties as derived from experiments on isolated cardiac muscle, ${ }^{5,42}$ i.e., velocity of sarcomere shortening increases with passive stretch, strength of activation increases with sarcomere length, and duration of activation increases with sarcomere length. Using myofiber stress and wall geometry, total radial and axial force components of tension acting on the junction are calculated. Then, septal geometry (initially estimated) is adjusted so that equilibrium of tensional force in the junction is satisfied. As a result, geometries of the three ventricular walls are known, together with myofiber stresses, wall tensions, and ventricular cavity volumes and pressures. Summarizing, the TriSeg model relates global LV and RV pump mechanics to local myofiber mechanics in the three ventricular walls. This relation is based on the principle of conservation of energy, meaning that summed ventricular pump work equals summed mechanical work as generated by the myofibers in the ventricular walls.

The CircAdapt model of the whole circulation ${ }^{1}$ creates the required physiological environment (circulatory boundary conditions) for the ventricles as represented by the TriSeg model. The CircAdapt model consists of a network of modules representing myocardial walls, valves, large blood vessels, and peripheral resistances. It allows beat-to-beat simulation of time-dependent ventricular mechanics and hemodynamics, e.g., volumes and pressures of the ventricular cavities, geometries and myofiber mechanics of the three ventricular walls, and flows through valves. An important feature of the CircAdapt model is that the number of independent parameters is reduced by incorporating adaptation of cardiac and vascular wall size and wall mass to mechanical load so that stresses and strains in the walls of heart and blood vessels are normalized to tissue-specific physiological standard levels. ${ }^{1,25}$

Pulmonary peripheral resistance is modeled as a non-linear resistive module representing the pulmonary microcirculation and connecting the compliant pulmonary arterial and venous blood vessel modules. ${ }^{1}$ Instantaneous blood flow through the pulmonary resistance $\left(q_{\text {pulm }}(t)\right.$, with $t$ representing time) is related to the squared instantaneous pulmonary arteriovenous pressure drop $\left(\Delta p_{\text {pulm }}(t)\right.$, defined as pulmonary artery pressure - pulmonary venous pressure):

$$
\mathrm{q}_{\text {pulm }}(\mathrm{t})=\left(\frac{\Delta \mathrm{p}_{\text {pulm }}(\mathrm{t})}{\Delta \mathrm{p}_{\text {pulm,mean }}}\right)^{2} \cdot \mathrm{CO}
$$

where constant parameters $\Delta \mathrm{p}_{\text {pulm,mean }}$ and CO represent mean pulmonary arteriovenous pressure drop and cardiac output, respectively. The non-linearity 
of this pressure-flow relationship is in agreement with the one measured in the human and canine lung with normal as well as increased pulmonary peripheral resistance due to pulmonary vascular disease. ${ }^{13,15,45}$ Both measured and simulated pulmonary pressure-flow relationships show strong non-linearity at low flow. This non-linearity at low flow is mainly caused by recruitment of pulmonary capillaries, i.e., with rising pressure, more capillaries begin to conduct blood, thus lowering overall resistance. At high flow, most pulmonary capillaries are recruited so that overall resistance changes little. The latter change of resistance in the absence of recruitment is a result of vessel distension.

Instantaneous blood flow $\mathrm{q}_{\text {sys }}(\mathrm{t})$ through the systemic resistance is directly proportional to the systemic arteriovenous pressure drop $\Delta \mathrm{p}_{\text {sys }}$ (aortic pressure - systemic venous pressure):

$$
\mathrm{q}_{\mathrm{sys}}(\mathrm{t})=\frac{\Delta \mathrm{p}_{\text {sys }}(\mathrm{t})}{\Delta \mathrm{p}_{\text {sys, mean }}} \cdot \mathrm{CO}
$$

where $\Delta \mathrm{p}_{\mathrm{sys}, \text { mean }}$ represents mean systemic arteriovenous pressure drop defined as mean arterial pressure (MAP) minus instantaneous systemic venous pressure.

The combined CircAdapt/TriSeg model was used to simulate cardiovascular mechanics and hemodynamics under normal healthy ventricular loading conditions (Normal simulation) and under chronic thromboembolic pulmonary hypertensive conditions before (CTEPH simulation) and after (POST-PTE simulation) pulmonary thromboendarterectomy. Furthermore, simulations were performed to quantify the effects of either decrease of MPAP (POST-mPAP simulation) or increase of CO (POST-CO simulation) on LV filling hemodynamics in CTEPH.

In each simulation, systemic venous return is set to pre- and postoperatively measured values of $\mathrm{CO}$ (Table 6.2), whereas $\Delta p_{\text {pulm,mean }}$ is chosen so that simulated mPAP equals pre- and postoperatively measured values (Table 6.2). In all simulations, MAP and heart rate are set to their preoperatively measured values (93 $\mathrm{mmHg}$ and $80 \mathrm{bpm}$, respectively) because both MAP and heart rate were not significantly different after PTE (Table 6.1).

\section{Normal simulation}

The Normal simulation rendered human cardiovascular mechanics and hemodynamics under normal ventricular loading conditions at rest. Size and mass of cardiac walls and large blood vessels were adapted to normalize mechanical load in the tissues. The methods used for simulation of normal ventricular mechanics and hemodynamics have been published in detail 
elsewhere.25 In the Normal simulation, CO was set to the average value measured postoperatively in the CTEPH patients (Tables 6.1 and 6.2).

Figure 6.1 shows a schematic representation of the simulation protocol as followed to simulate the different patient conditions as described below.

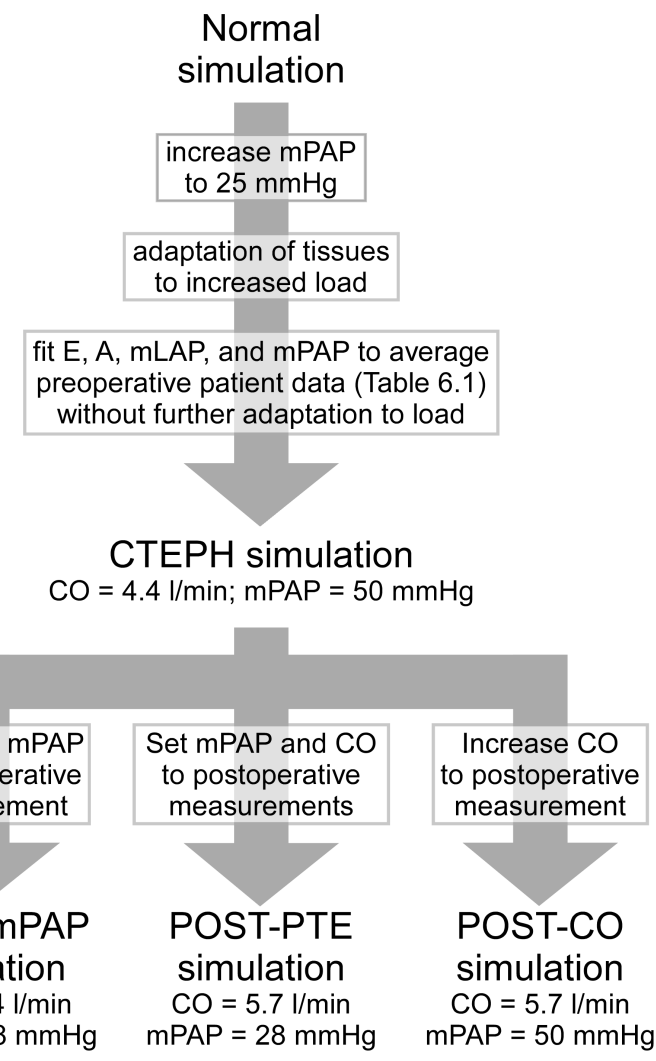

Figure 6.1: Schematic representation of the simulation protocol. Starting from a reference simulation of normal cardiovascular mechanics and hemodynamics (Normal), the model was fitted to average hemodynamic data measured preoperatively in a group of CTEPH patients (CTEPH simulation). Pulmonary thromboendarterectomy (PTE) was simulated by acutely changing mean pulmonary artery pressure (mPAP) and cardiac output (CO) to average postoperative measurements (POST-PTE simulation). The effect of RV afterload on LV filling hemodynamics in CTEPH patients undergoing PTE was quantified by decreasing mPAP to postoperative value, while keeping CO constant (POST-mPAP simulation). Similarly, to quantify the effect of RV systolic output on LV filling, CO was increased from pre- to postoperative value, while keeping mPAP constant (POST-CO simulation). Abbreviations: $A$ = peak blood flow velocity of the late mitral filling wave; $E=$ peak blood flow velocity of the early mitral filling wave; and $\mathrm{mLAP}=$ mean left atrial pressure. 


\section{CTEPH simulation}

Using the Normal simulation as point of departure, the CTEPH simulation was obtained in two steps. First, PVR was increased so that mPAP was equal to 25 $\mathrm{mmHg}$. Up to this level of RV afterload, we assumed the heart and large blood vessels to be able to adapt their wall size and wall mass so that stresses and strains in the walls are normalized to tissue-specific physiological levels. Moreover, this value of mPAP was chosen because pulmonary hypertension is clinically defined as mPAP $>25 \mathrm{mmHg}$ at rest. ${ }^{11}$ The adaptation led to a $25 \%$ increase of the mass of the RV free wall. Second, several model parameters were varied so that simulated sum of mitral E- and A-velocities, mitral E/A, mean left atrial pressure (mLAP), and mPAP matched the average measurements as obtained preoperatively in the patients (Table 6.1), i.e.,

- $\quad$ mitral valve area was varied so that the sum of mitral E- and A-velocities was $1.21 \mathrm{~m} / \mathrm{s}$;

- $\quad$ passive stiffness of the left atrial myocardium was varied so that mitral E/A was 0.74;

- $\quad$ passive stiffness of the LV myocardium (LV free wall and septum) was varied so that $\mathrm{mLAP}$ was $9.8 \mathrm{mmHg}$; and

- $\Delta p_{\text {pulm,mean }}$ was varied so that mPAP was $50 \mathrm{mmHg}$.

During the fitting procedure, no further adaptation of cardiac and vascular walls to mechanical load was performed. Similarly as observed in previous studies, ${ }^{23,} 25$ increase of mPAP from 25 to $50 \mathrm{mmHg}$ without adaptation led to $\mathrm{RV}$ decompensation, i.e., increase of RV end-diastolic volume and of RV filling pressure (Table 6.2). It was assumed that pulmonary capillary wedge pressure was a reasonable accurate surrogate of mLAP in the present patient group. ${ }^{2}$ The resulting CTEPH simulation served as reference state for each of the remaining three simulations, i.e., POST-PTE, POST-mPAP, and POST-CO.

\section{POST-PTE, POST-MPAP, and POST-CO simulations}

The POST-PTE simulation, representing the average CTEPH patient after PTE, was obtained by applying two acute interventions (Figure 6.1), as derived from the patient data (Table 6.1): 1) $\Delta p_{\text {pulm,mean }}$ was decreased so that mPAP decreased from $50 \mathrm{mmHg}$ to $28 \mathrm{mmHg}$ and 2) CO was increased from $4.4 \mathrm{l} / \mathrm{min}$ to 5.7 $\mathrm{I} / \mathrm{min}$. The POST-mPAP simulation was obtained by decreasing mPAP to postoperative value, while CO was set to preoperative value. Similarly, the POST$\mathrm{CO}$ simulation was obtained by increasing $\mathrm{CO}$ to postoperative value, while mPAP was kept constant at preoperative value. 


\section{Assessment of LV filling and septal geometry}

Starling function curves are obtained by plotting CO, which reflects LV output, as function of mLAP, which approximates LV filling pressure. ${ }^{34}$ The Starling curve is afterload dependent and characterizes ventricular function and interaction of the ventricle with the distal vascular system. Under the condition of constant LV pressure load, a shift up and to the left of the LV Starling curve generally indicates improved LV function, with greater output for a given filling pressure. In the present study, Starling curves of the left heart were obtained by variation of $\mathrm{CO}$ between 3 and $7 \mathrm{l} / \mathrm{min}$ with high preoperative mPAP and with low postoperative mPAP, to asses the effect of RV afterload on left heart function as transmitted by the septum.

To assess the effect of PTE on septal geometry, average septal curvature during the early mitral filling wave $\left(C_{\text {sept }}\right)$ was quantified. This curvature was defined as the reciprocal of radius of curvature and was defined positive for physiological septal bulging towards the RV cavity.

\section{Sensitivity analysis}

The CTEPH, the POST-MPAP, and the POST-CO simulations were used to quantify the relative sensitivity (RS) of the dependent parameter DP for changes of the independent parameters mPAP and CO. For example, $\mathrm{RS}_{\mathrm{DP} \text {, mPAP }}$ indicates relative sensitivity of DP for changes of MPAP:

$$
\mathrm{RS}_{\text {DP, MPAP }}=\frac{\log \left(\frac{\mathrm{DP}_{\text {POST-MPAP }}}{\text { DP }_{\text {CTEPH }}}\right)}{\log \left(\frac{\mathrm{mPAP} P_{\text {POST-MPAP }}}{m \text { PAP }_{\text {CTEPH }}}\right)}
$$

where the denominator is a constant value of -0.58 . Analogously, RSDP, CO indicates relative sensitivity of DP for changes of $\mathrm{CO}$ :

$$
\mathrm{RS}_{\mathrm{DP}, \mathrm{CO}}=\frac{\log \left(\frac{\mathrm{DP} \mathrm{P}_{\text {POST-CO }}}{\mathrm{DP}_{\text {CTEPH }}}\right)}{\log \left(\frac{C \mathrm{CO}_{\text {POST-CO }}}{\mathrm{CO}_{\text {CTEPH }}}\right)}
$$

where the denominator is a constant value of 0.26 . A value of 1,0 , and -1 for RS indicates a proportional, independent, and reciprocal relationship, respectively. Septal curvature can be zero or negative with pulmonary hypertension. Consequently, RS could not be quantified for $C_{\text {sept }}$ because only positive real numbers have real-valued logarithms. 


\section{RESULTS}

Figure 6.2 shows measured as well as simulated LV filling parameters in CTEPH patients before and after PTE. Similarly as measured in patients, the model predicted increase of mitral E-velocity, mitral E/A, mLAP, and little change of mitral A-velocity with PTE in CTEPH patients. Simulation data indicate that the increase of mitral E/A is predominantly caused by increase of $\mathrm{CO}$ (Figure 6.2C, blue line) and to a lesser degree by decrease of mPAP (Figure 6.2C, red line). Increase of CO and decrease of mPAP have opposite effects on mLAP (Figure 6.2D). The increase of $\mathrm{mLAP}$ with $\mathrm{CO}$ (Figure 6.2D, blue line) is much larger than the decrease with mPAP (Figure 6.2D, red line) resulting in a net increase of mLAP with PTE.
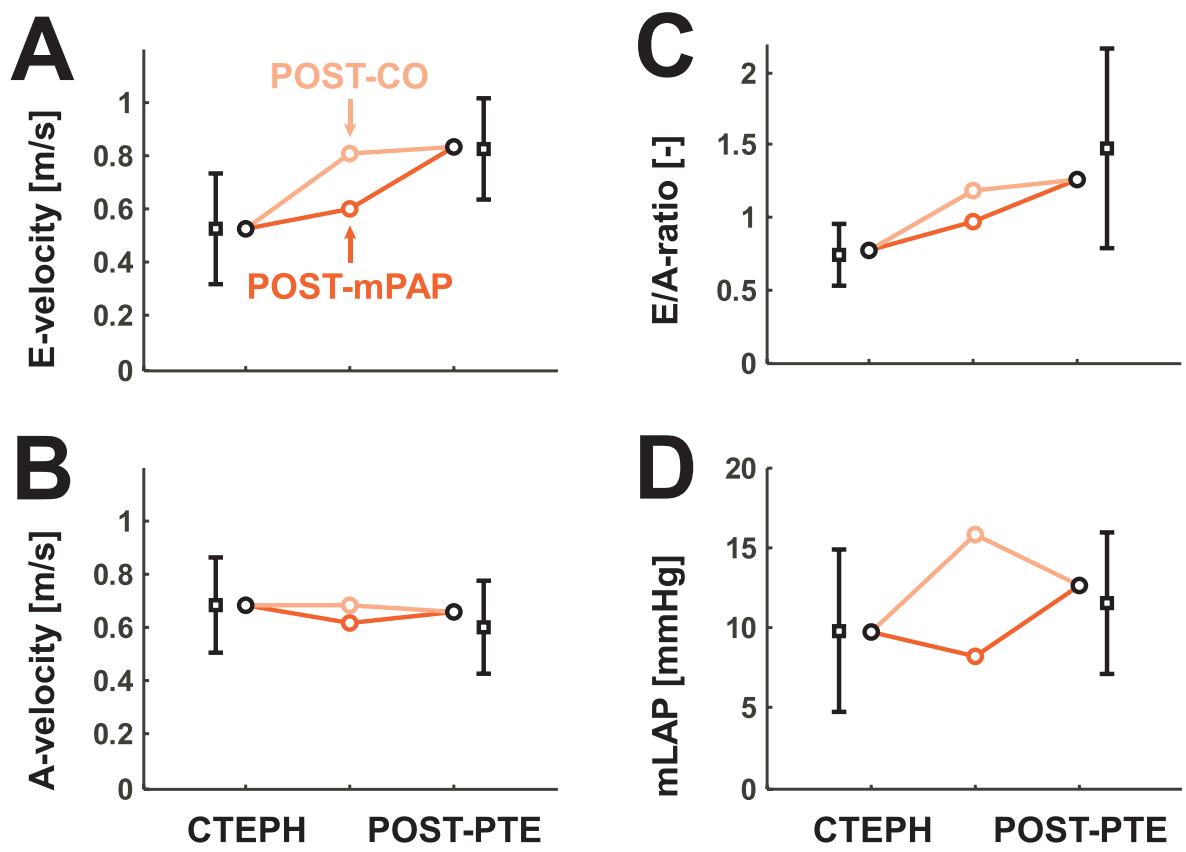

Figure 6.2: LV filling parameters in CTEPH patients before and after PTE: measurements and simulations. Squares with error bars represent measurements (mean \pm standard deviation) in CTEPH patients. Circles represent data points derived from the simulations as defined in Figure. 6.1. A: peak mitral E-velocity. B: peak mitral A-velocity. C: mitral E/A-ratio. D: mean left atrial pressure (mLAP). 
Time courses of simulated mitral and aortic blood flow velocities (Figure 6.3A) show that PTE predominantly affected the mitral E-wave and hardly affected the A wave. Increase of CO (Figure 6.3A, blue line) caused most of the increase of the E-wave, whereas decrease of MPAP (Figure 6.3A, red line) caused a relatively small shift of flow from the late A-wave to the early E-wave. Increase of $\mathrm{CO}$ as well as decrease of mPAP resulted in earlier opening of the mitral valve. PTE did not affect timing of mitral and aortic valve closure. Consequently, duration of the LV isovolumic relaxation phase decreased from $124 \mathrm{~ms}$ (CTEPH) to $90 \mathrm{~ms}$ (POST-PTE). Furthermore, PTE resulted in relatively little changes of duration of LV ejection (increase from $234 \mathrm{~ms}$ to $244 \mathrm{~ms}$ ) and of LV isovolumic contraction (decrease from $46 \mathrm{~ms}$ to $34 \mathrm{~ms}$ ). LV Tei (or myocardial performance) index, defined as the sum of LV isovolumic contraction and relaxation time divided by LV ejection time ${ }^{41}$ decreased significantly from 0.73 to 0.51 .

Time courses of simulated septal curvature (Figure 6.3B) show that the interventricular septum is relatively flat in the CTEPH simulation. After aortic valve closure, septal curvature decreases quickly to negative value at moment of mitral valve opening. This decrease of septal curvature corresponded with a rapid leftward septal motion during the LV isovolumic relaxation phase. As a result, the septum bulges towards the LV cavity during early LV filling. In the POST-PTE simulation, septal curvature was increased to nearly normal values. The increase of septal curvature with PTE resulted almost entirely from decrease of mPAP rather than from increase of $\mathrm{CO}$. The effect of PTE on septal motion is clearly illustrated by supplemental echocardiographic movies showing ventricular geometry in parasternal short axis view as obtained pre- and postoperatively in a CTEPH patient. The first movie (Data supplement: Movie I) demonstrates a flat interventricular septum and rapid end-systolic leftward septal motion in a CTEPH patient before PTE. The second movie (Data supplement: Movie II) demonstrates abolishment of septal flattening and leftward septal motion after PTE. For qualitative comparison with these measurements, two additional movies demonstrate simulated ventricular geometry and time courses of ventricular pressures as obtained from the CTEPH and POST-PTE simulations (Data supplement: Movie III and Movie IV, respectively).

Table 6.2 contains the values of several hemodynamic and geometric parameters in all simulations. These data show that mitral E/A, mitral E-velocity, $\mathrm{C}_{\text {sept, }}$ LV end-diastolic volume, and LV as well as RV ejection fraction increased from subnormal values in the CTEPH simulation to normal values in the POST-PTE simulation. Similarly, RV end-diastolic volume decreased from a pathologically high value to a normal physiological one. However, mLAP did not decrease to a 


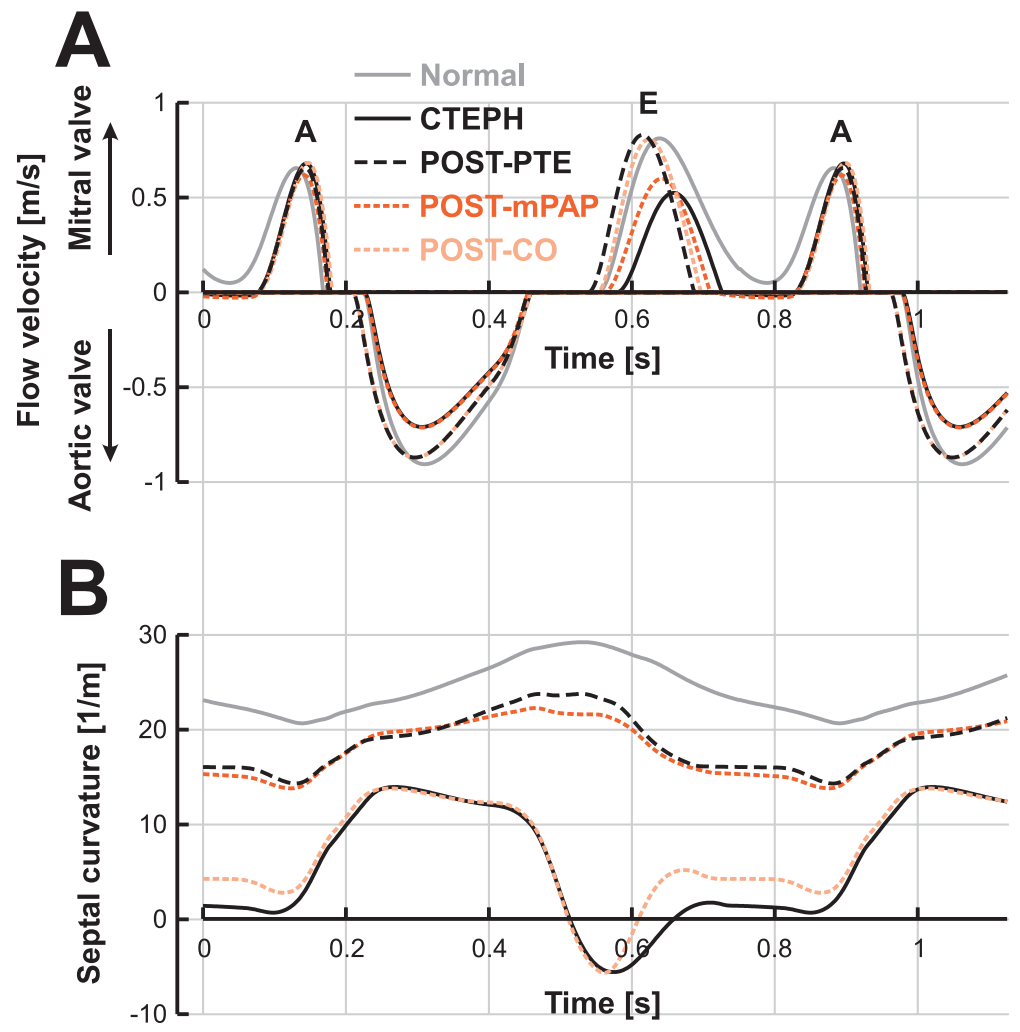

Figure 6.3: Simulated time courses of blood flow velocities in the mitral and aortic valves and of septal curvature. See Figure 6.1 for definitions of simulations. A: time courses of mitral (positive) and aortic (negative) blood flow velocities. B: time courses of septal curvature. Positive curvature denotes rightward septal curvature, whereas negative curvature denotes leftward septal curvature. Zero curvature represents a flat septum. Simulation duration amounts to 1.5 cardiac cycle. Abbreviations as in Figure 6.1.

normal physiological value but increased by $29 \%$ from CTEPH to POST-PTE. Moreover, mean right atrial pressure (mRAP) decreased $30 \%$.

Table 6.2 also presents sensitivity analysis data as derived from the POSTmPAP and POST-CO simulations. The POST-mPAP simulation revealed that mitral Evelocity increased less than proportional with decrease of mPAP, whereas the POST$\mathrm{CO}$ simulation revealed that mitral E-velocity increased more than proportional with $\mathrm{CO}$. Furthermore, mitral A-velocity decreased less than proportional with mPAP, whereas it was not affected at all by CO. Summarizing, these data suggest that, in 


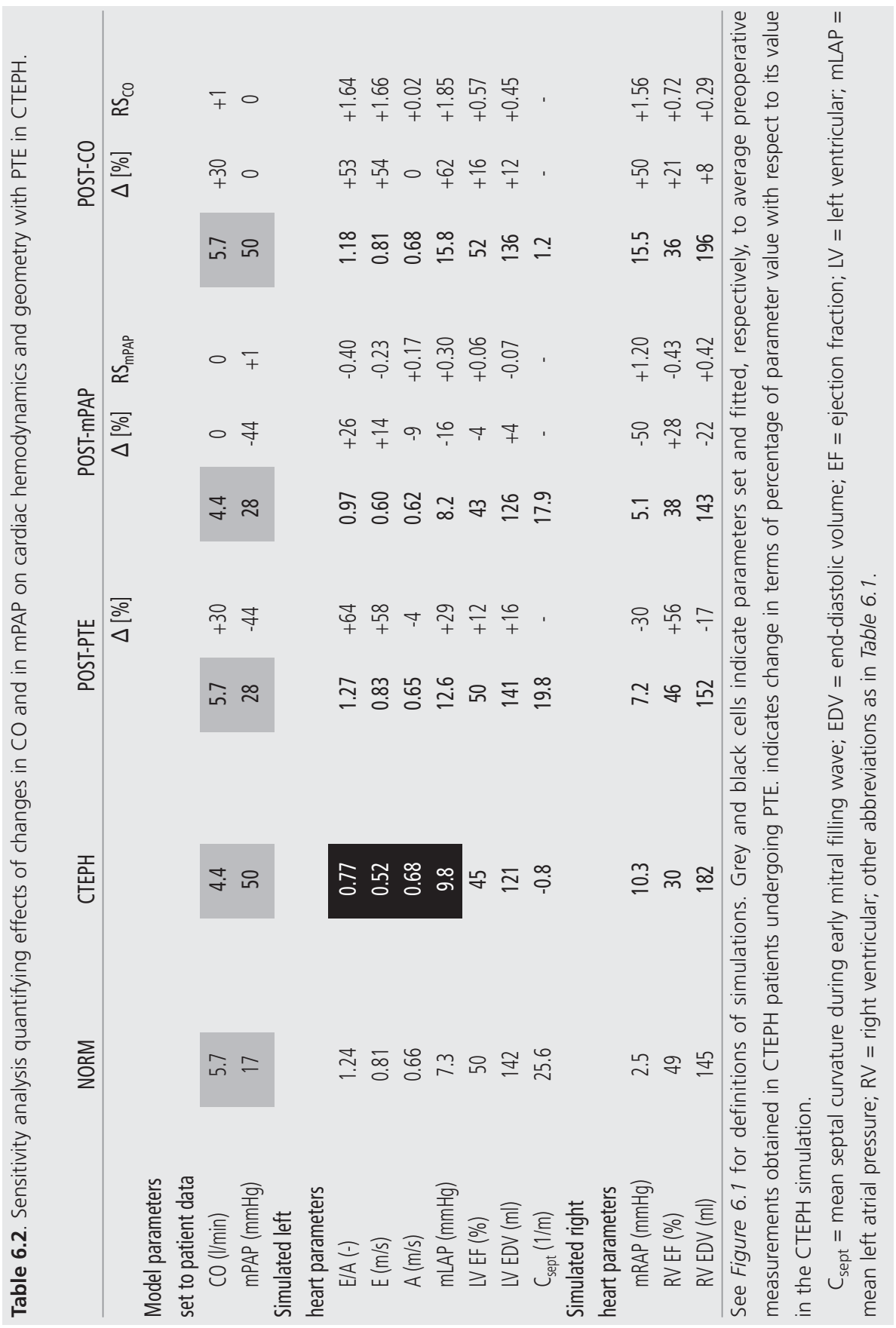


$\mathrm{CTEPH}$, mitral E/A is four times more sensitive to relative changes of $\mathrm{CO}\left(\mathrm{RS}_{\mathrm{CO}}=\right.$ $+1.64)$ than to those of mPAP $\left(R S_{\text {mPAP }}=0.40\right)$. Apparently, the increase of mitral E/A with PTE resulted predominantly from increase of RV systolic output and not so much from normalization of septal geometry.

Figure 6.4 shows LV Starling curves as obtained by variation of $\mathrm{CO}$ with high preoperative mPAP (solid line) and with low postoperative MPAP (dashed line), while LV afterload was kept constant. Decrease of mPAP resulted in a shift of the LV Starling curve to the left indicating improvement of LV performance. In the CTEPH patients, however, mLAP increased with PTE (Figure 6.4: from closed to

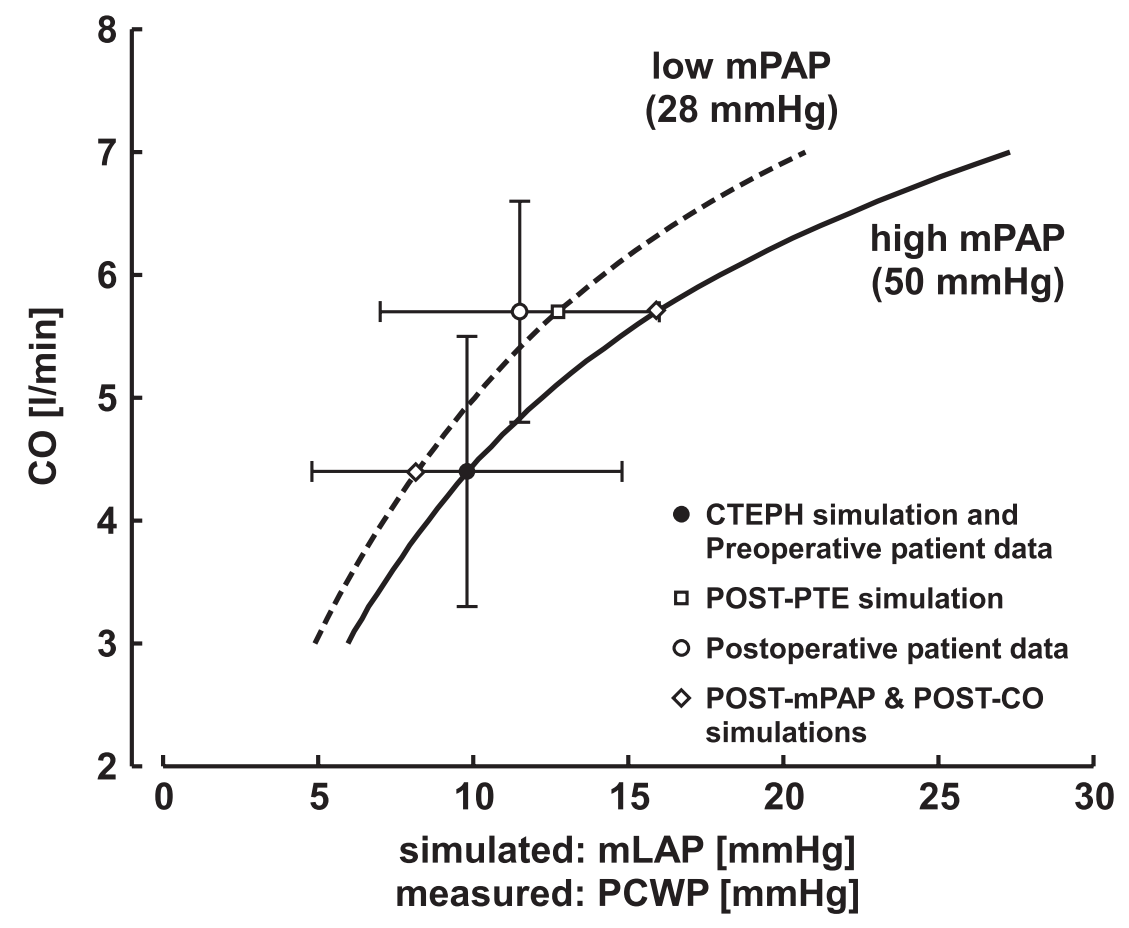

Figure 6.4: Simulated left heart function curves with high preoperative MPAP (solid line) and with low postoperative MPAP (dashed line). Crosses indicate pre- and postoperative average values and their standard deviations as measured in the CTEPH patients. Data points obtained from the POST-mPAP simulation (open diamond on dashed line) and POST-CO simulation (open diamond on solid line) are indicated. Note that measured pulmonary capillary wedge pressure (PCWP) is assumed to be a reasonable accurate surrogate of mean left atrial pressure (mLAP) in the present patient group. Abbreviations as in Figure 6.1. 
open circle). When exclusively increasing CO in our model (POST CO simulation) to average postoperative value (5.7 l/min), mLAP increased $62 \%$ (Figure 6.4: from closed circle to open diamond on solid line). When changing both MPAP and CO to average postoperative values (Figure 6.4: from closed circle to open square on dashed line), mLAP increased $29 \%$ to a value close to the actual one measured postoperatively in the patients (Figure 6.4: open circle).

\section{Discussion}

In the present study, we assessed the effect of PTE on LV filling mechanics and hemodynamics in CTEPH patients by fitting a mathematical model of cardiovascular mechanics and hemodynamics including ventricular interaction to measurements obtained in a group of CTEPH patients before and after PTE. The model was made pathology-specific by fitting it to average preoperative patient data. Starting from this preoperative reference state, PTE was simulated by decreasing MPAP and by increasing $\mathrm{CO}$ acutely to average postoperative values. Simulated postoperative LV filling hemodynamics was in agreement with postoperative measurements (Figure 6.2). The added value of our modeling approach was that the effect of leftward septal bulging and the effect of $\mathrm{CO}$ on LV filling could be separated quantitatively by exclusively decreasing mPAP and by exclusively increasing $\mathrm{CO}$ to postoperative values, respectively. The latter model simulations suggested that 1) mitral E/A was about four times more sensitive to relative changes of $\mathrm{CO}$ than to those of mPAP, 2) mitral A-velocity was relatively insensitive to acute changes of $\mathrm{CO}$ and MPAP, and 3) septal curvature during early LV filling was very sensitive to changes of MPAP and relatively insensitive to changes of $\mathrm{CO}$ (Table 6.2). These simulation data suggest that increase of mitral E/A with PTE in CTEPH patients is largely the effect of increased RV systolic output and that abolishment of leftward septal bulging is of minor importance.

The acute effect of PTE on LV filling hemodynamics as predicted by our model was qualitatively as well as quantitatively similar to the effect as measured postoperatively in the CTEPH patients (Figure 6.2), whose average preoperative data were used as input for our model. Mitral E/A, mitral E-velocity, and $\mathrm{mLAP}$ were increased while mitral A-velocity was relatively unaffected after PTE. The simulated effect of PTE on ventricular cavity volumes is also in close agreement with the effect as derived from pre- and postoperative MRI measurements in CTEPH patients undergoing PTE. ${ }^{35}$ These measurements as well as our simulations show that, under CTEPH conditions before PTE, LV end- 
diastolic volume, LV ejection fraction, and RV ejection fraction are significantly decreased whereas RV end-diastolic volume is significantly increased with respect to values simulated and measured under normal physiological conditions. Furthermore, simulated as well as measured ventricular end-diastolic volumes and ejection fractions changed to normal physiological values after PTE. ${ }^{35}$ In our simulations as well as in patients with CTEPH, ${ }^{30}$ the favorable effect of successful PTE on global LV performance is reflected by significant decrease of LV Tei index. Also, septal geometry was simulated realistically before and after PTE. Similarly as observed in CTEPH patients, ${ }^{16,35}$ our simulations showed a major change of septal curvature with PTE (Figure 6.3B and Table 6.2), i.e., from negative septal curvature (leftward septal bulging) to physiological positive septal curvature (rightward septal bulging) after aortic valve closure and during early LV filling. Since septal geometry directly depends on the transseptal pressure gradient, ${ }^{8}$ decrease of MPAP rather than increase of $\mathrm{CO}$ resulted in abolishment of leftward septal bulging by re-establishment of the normal negative transseptal pressure gradient (LV pressure $>$ RV pressure). Supplemental movies show strong similarity between simulated and measured septal motion before and after PTE in CTEPH (Data supplement: Movies I-IV). All these similarities between simulations and measurements show that our model enables realistic simulation of ventricular mechanics and hemodynamics under pre- and postoperative CTEPH conditions.

The leftward shift of the LV Starling curve with decrease of MPAP (Figure 6.4) indicates that the LV fills at lower diastolic pressure. This finding is in agreement with experiments in cardiac preparations in which direct mechanical ventricular interaction via the septum had been preserved but series ventricular interaction via the pulmonary circulation had been abolished. ${ }^{9,} 32,40,44$ These studies showed that decrease of RV afterload decreased LV end diastolic pressure as a direct result of geometrical changes such as reduction of leftward septal bulging. LV afterload and filling volume were kept constant during these experiments.

In addition to these experimental data, our POST-mPAP and POST-CO simulations show how a decrease of RV afterload and how an increase of RV systolic output, respectively, affect clinical LV filling parameters as measured in CTEPH patients. In the POST-mPAP simulation, decrease of MPAP was associated with $9 \%$ decrease of mitral A-velocity and with $14 \%$ increase of mitral $E$ velocity. Apparently, the acute abolishment of leftward bulging of the interventricular septum facilitates passive LV filling (increase mitral E-wave) and results in redistribution of blood flow from the late to the early mitral filling 
phase evidenced by a $26 \%$ increase of mitral E/A in the POST-mPAP simulation (Figures 6.2 and 6.3). In the POST-CO simulation, however, increase of CO did not affect mitral A-velocity but resulted in 54\% increase of mitral E-velocity. In CTEPH patients, PTE resulted in a small (not significant) decrease of mitral Avelocity and in a significant increase of pulmonary capillary wedge pressure (Table 6.1). ${ }^{12,27}$ In combination with these latter observations in patients, our simulations show that decrease of RV afterload and associated abolishment of leftward septal bulging, could only explain a relatively small part $(\sim 30 \%)$ of the increase of mitral E/A observed in patients undergoing PTE. Increase of CO, however, accounted for most ( 70\%) of the increase of E/A with PTE.

Our finding that an exclusive $30 \%$ increase of CO (POST-CO simulation) did not affect mitral A-velocity and resulted in $54 \%$ increase of mitral E-velocity is in agreement with Doppler measurements obtained before and after hemodialysis in patients with renal insufficiency but without overt heart disease. ${ }^{39}$ In these patients, the decrease of circulating blood volume as a result of hemodialysis is associated with a $13 \%$ decrease of CO, whereas heart rate and systemic blood pressures did not change significantly. This decrease of $\mathrm{CO}$ was associated with $\sim 30 \%$ decrease of mitral E-velocity and mitral E/A, whereas mitral A-velocity remained unchanged. Although pulmonary artery pressure and septal geometry are unknown in this patient group, it is very unlikely that these parameters are significantly different before and after hemodialysis. Therefore, these data strongly support our conclusion derived from our simulations of PTE, i.e., increase of mitral E/A with PTE predominantly results from increase of LV preload rather than from release of LV compression by the septum.

In animal models with chronic RV pressure overload, LV systolic function (contractility) appeared unaffected. ${ }^{10,17,18,20}$ Thus, increase of CO with PTE is most likely the result of improved RV systolic function. This latter improvement may have several causes. First, decrease of RV afterload in decompensated pulmonary hypertension results in reduction of interventricular mechanical dyssynchrony ${ }^{23,26,28}$ and, thus, in a more coordinated contraction of the three ventricular walls with less leftward septal bulging. This may, therefore, account for an indirect effect of decreased leftward septal bulging on LV filling hemodynamics via improvement of RV systolic output. Second, in patients with CTEPH, decrease of RV afterload by PTE has been shown to significantly reduce tricuspid regurgitation. ${ }^{36}$ Third, PTE and the associated decrease of RV enddiastolic volume may result in an acute reduction of total heart volume and, thus, of pericardial constraint. Acute reduction of pericardial constraint has been shown to increase $\mathrm{CO}$ in various animal models of pulmonary hypertension..$^{3,4}$ 
Finally (and speculatively), PTE may lead to improvement of RV coronary perfusion. Exorbitantly high RV pressure may increase intramyocardial pressure in the RV free wall and, thus, increase resistance of the RV coronary vasculature more than that of the LV. This may result in relatively reduced myocardial perfusion of the RV. The acute decrease of RV pressure with PTE may result in a decrease of RV coronary vascular resistance (facilitating RV myocardial perfusion) and also cause a decrease in RV mechanical myofiber load. Consequently, RV contractile function and stroke volume can improve.

Many studies have reported strong correlations between septal curvature and LV filling parameters such as E/A and speculated on the potential detrimental effect of leftward septal bulging on LV filling in patients with

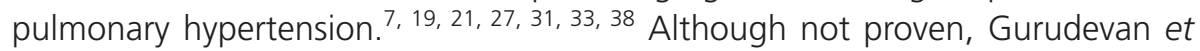
al. ${ }^{12}$ suggested that increase of mitral E/A with PTE predominantly resulted from increase of LV preload rather than from release of LV compression by the septum. However, the relative contributions of both mechanisms could not be quantified from the available patient data alone. Our simulations confirm the hypothesis that increase of mitral E/A with PTE in CTEPH patients is largely the effect of increased RV systolic output while abolishment of leftward septal bulging is of minor importance. However, whether normalization of septal geometry and motion also has an indirect effect on LV filling by increasing RV performance and, thus, RV systolic output, remains unknown. This cannot be tested using our model.

\section{Clinical implications}

There has been some debate concerning the relative importance of LV underfilling vs. LV compression by septal bulging in the low-output state of advanced pulmonary hypertension. ${ }^{12,29}$ The etiology of the LV "diastolic dysfunction" often present in CTEPH has also been controversial. ${ }^{22,27,37}$ The results of the present study strongly support the concept that perturbations in LV filling pressure, output, and Doppler flow patterns due to CTEPH are primarily caused by RV outflow obstruction and resultant LV underfilling. Although not negligible, the relative contribution of septal geometry and bulging is considerably less. Also, the increases in cardiac output and LV filling explain the marked improvement in LV Doppler diastolic filling pattern after PTE, rather than changes in septal geometry per se. 


\section{Study limitations}

Obviously, conditions in a computer simulation may differ from those in patients. An important limitation of our model is the fact that heart rate, ventricular stroke volume, and, hence, cardiac output are prescribed independent model parameters. In reality, however, these parameters are mutually dependent and are tightly controlled in order to maintain mean arterial blood pressure. Thus, the exact mechanism by which PTE results in increase of $\mathrm{CO}$ can not be studied with the current model. Nevertheless, the quantitative agreement between our postoperative simulation data and postoperative patient data on LV filling hemodynamics (Figure 6.2) indicate that the simplifications made in the simulations may not obscure firstorder mechanical effects of hemodynamic and mechanical ventricular interaction on LV filling characteristics in CTEPH patients undergoing PTE.

Although the supplemental movies show qualitative agreement between simulated and measured changes of septal motion due to PTE, septal curvature is not quantified in our patients. Nevertheless, in a previous study, ${ }^{25}$ we have shown that the relation between septal curvature and transseptal pressure difference in our model is similar to the relation measured in a group of healthy volunteers and patients with pulmonary arterial hypertension. ${ }^{6}$

Tricuspid regurgitation, which often exists in patients with CTEPH and which has been shown to be significantly reduced by PTE, ${ }^{36}$ is not included in our simulations.

\section{CONCLUSIONS}

The effect of PTE on LV filling hemodynamics as predicted by our model was surprisingly similar to the effect as observed in patients with CTEPH. Mitral E/A, mitral E-velocity, LV end-diastolic volume, and septal curvature increased from subnormal values in CTEPH before PTE to normal values after PTE, whereas RV end-diastolic volume decreased to normal value. Our model simulations suggest that 1) mitral E/A is about four times more sensitive to relative changes of cardiac output than to those of mean pulmonary artery pressure, 2) mitral Avelocity is relatively insensitive to changes of cardiac output and mean pulmonary artery pressure, and 3) septal curvature is almost solely dependent of mean pulmonary artery pressure and relatively independent of cardiac output. Our simulation data suggest that one-third of the increase of mitral E/A with PTE in CTEPH patients can be explained by the mechanical effect of decrease of RV pressure overload as transmitted by the septum. The remaining two-third increase of mitral E/A can be attributed to increase of RV systolic output. 


\section{GRANTS}

This work was financially supported by Actelion Pharmaceuticals Nederland B.V. (Woerden, The Netherlands) and in part by the Netherlands Heart Foundation (Grant 2007B203).

\section{Disclosures}

We, the authors of this manuscript, state that there are no conflicts of interest to disclose. 
Chapter 6 


\section{REFERENCES}

1. Arts T, Delhaas T, Bovendeerd P, Verbeek X, and Prinzen FW. Adaptation to mechanical load determines shape and properties of heart and circulation: The circadapt model. Am J Physiol Heart Circ Physiol 288: H1943-1954, 2005.

2. Batson GA, Chandrasekhar KP, Payas $Y$, and Rickards DF. Measurement of pulmonary wedge pressure by the flow directed swan-ganz catheter. Cardiovasc Res 6: 748-752, 1972.

3. Belenkie I, Dani R, Smith ER, and Tyberg JV. The importance of pericardial constraint in experimental pulmonary embolism and volume loading. Am Heart J 123: 733-742, 1992.

4. Belenkie I, Sas R, Mitchell J, Smith ER, and Tyberg JV. Opening the pericardium during pulmonary artery constriction improves cardiac function. J Appl Physiol 96: 917-922, 2004.

5. de Tombe PP, and ter Keurs HE. Force and velocity of sarcomere shortening in trabeculae from rat heart. Effects of temperature. Circ Res 66: 1239-1254, 1990.

6. Dellegrottaglie S, Sanz J, Poon M, Viles-Gonzalez JF, Sulica R, Goyenechea M, Macaluso F, Fuster V, and Rajagopalan S. Pulmonary hypertension: Accuracy of detection with left ventricular septal-to-free wall curvature ratio measured at cardiac mr. Radiology 243: 63-69, 2007.

7. Dittrich HC, Chow LC, and Nicod PH. Early improvement in left ventricular diastolic function after relief of chronic right ventricular pressure overload. Circulation 80: 823830, 1989.

8. Dong SJ, Smith ER, and Tyberg JV. Changes in the radius of curvature of the ventricular septum at end diastole during pulmonary arterial and aortic constrictions in the dog. Circulation 86: 1280-1290, 1992.

9. Elzinga G, van Grondelle R, Westerhof N, and van den Bos GC. Ventricular interference. Am J Physiol 226: 941-947, 1974.

10. Faber MJ, Dalinghaus M, Lankhuizen IM, Steendijk P, Hop WC, Schoemaker RG, Duncker DJ, Lamers JM, and Helbing WA. Right and left ventricular function after chronic pulmonary artery banding in rats assessed with biventricular pressure-volume loops. Am J Physiol Heart Circ Physiol 291: H1580-1586, 2006.

11. Galie N, Torbicki A, Barst R, Dartevelle P, Haworth S, Higenbottam T, Olschewski H, Peacock A, Pietra G, Rubin L, Simonneau G, Priori SG, Garcia MA, Blanc JJ, Budaj A, Cowie M, Dean V, Deckers J, Burgos EF, Lekakis J, Lindahl B, Mazzotta G, McGregor K, Morais J, Oto A, Smiseth OA, Barbera JA, Gibbs S, Hoeper M, Humbert M, Naeije R, and Pepke-Zaba J. Guidelines on diagnosis and treatment of pulmonary arterial hypertension. The task force on diagnosis and treatment of pulmonary arterial hypertension of the european society of cardiology. Eur Heart J 25: 2243-2278, 2004. 


\section{Chapter 6}

12. Gurudevan SV, Malouf PJ, Auger WR, Waltman TJ, Madani M, Raisinghani AB, DeMaria $A N$, and Blanchard DG. Abnormal left ventricular diastolic filling in chronic thromboembolic pulmonary hypertension: True diastolic dysfunction or left ventricular underfilling? J Am Coll Cardiol 49: 1334-1339, 2007.

13. Janicki JS, Weber KT, Likoff MJ, and Fishman AP. The pressure-flow response of the pulmonary circulation in patients with heart failure and pulmonary vascular disease. Circulation 72: 1270-1278, 1985.

14. Keogh AM, Mayer E, Benza RL, Corris P, Dartevelle PG, Frost AE, Kim NH, Lang IM, Pepke-Zaba J, and Sandoval J. Interventional and surgical modalities of treatment in pulmonary hypertension. J Am Coll Cardiol 54: S67-77, 2009.

15. Kondo M, Washizu M, Matsukura Y, Washizu T, Miyasaka K, and Takata M. Pressureflow relationship and longitudinal distribution of pulmonary vascular resistance in heartworm-infected dogs. J Vet Med Sci 65: 965-970, 2003.

16. Kreitner KF, Ley S, Kauczor HU, Mayer E, Kramm T, Pitton MB, Krummenauer F, and Thelen M. Chronic thromboembolic pulmonary hypertension: Pre- and postoperative assessment with breath-hold mr imaging techniques. Radiology 232: 535-543, 2004.

17. Lamberts RR, Caldenhoven E, Lansink M, Witte G, Vaessen RJ, St Cyr JA, and Stienen GJ. Preservation of diastolic function in monocrotaline-induced right ventricular hypertrophy in rats. Am J Physiol Heart Circ Physiol 293: H1869-1876, 2007.

18. Lamberts RR, Vaessen RJ, Westerhof N, and Stienen GJ. Right ventricular hypertrophy causes impairment of left ventricular diastolic function in the rat. Basic Res Cardiol 102: 19-27, 2007.

19. Lazar JM, Flores AR, Grandis DJ, Orie JE, and Schulman DS. Effects of chronic right ventricular pressure overload on left ventricular diastolic function. Am J Cardiol 72: 1179-1182, 1993.

20. Leeuwenburgh BP, Helbing WA, Steendijk P, Schoof PH, and Baan J. Biventricular systolic function in young lambs subject to chronic systemic right ventricular pressure overload. Am J Physiol Heart Circ Physiol 281: H2697-2704, 2001.

21. Louie EK, Rich S, and Brundage BH. Doppler echocardiographic assessment of impaired left ventricular filling in patients with right ventricular pressure overload due to primary pulmonary hypertension. J Am Coll Cardiol 8: 1298-1306, 1986.

22. Louie EK, Rich S, Levitsky S, and Brundage BH. Doppler echocardiographic demonstration of the differential effects of right ventricular pressure and volume overload on left ventricular geometry and filling. J Am Coll Cardiol 19: 84-90, 1992.

23. Lumens J, Arts T, Broers B, Boomars KA, van Paassen P, Prinzen FW, and Delhaas T. Right ventricular free wall pacing improves cardiac pump function in severe pulmonary arterial hypertension: A computer simulation analysis. Am J Physiol Heart Circ Physiol 297: H2196-2205, 2009. 


\section{Left Ventricular Filling in Pulmonary Hypertension}

24. Lumens J, Delhaas T, Kirn B, and Arts T. Modeling ventricular interaction: A multiscale approach from sarcomere mechanics to cardiovascular system hemodynamics. Pac Symp Biocomput 378-389, 2008.

25. Lumens J, Delhaas T, Kirn B, and Arts T. Three-wall segment (TriSeg) model describing mechanics and hemodynamics of ventricular interaction. Ann Biomed Eng 37: 22342255, 2009.

26. Lurz P, Puranik R, Nordmeyer J, Muthurangu V, Hansen MS, Schievano S, Marek J, Bonhoeffer $\mathrm{P}$, and Taylor AM. Improvement in left ventricular filling properties after relief of right ventricle to pulmonary artery conduit obstruction: Contribution of septal motion and interventricular mechanical delay. Eur Heart J 30: 2266-2274, 2009.

27. Mahmud E, Raisinghani A, Hassankhani A, Sadeghi HM, Strachan GM, Auger W, DeMaria AN, and Blanchard DG. Correlation of left ventricular diastolic filling characteristics with right ventricular overload and pulmonary artery pressure in chronic thromboembolic pulmonary hypertension. J Am Coll Cardiol 40: 318-324, 2002.

28. Marcus JT, Gan CT, Zwanenburg JJ, Boonstra A, Allaart CP, Gotte MJ, and VonkNoordegraaf A. Interventricular mechanical asynchrony in pulmonary arterial hypertension: Left-to-right delay in peak shortening is related to right ventricular overload and left ventricular underfilling. J Am Coll Cardiol 51: 750-757, 2008.

29. Marcus JT, Vonk Noordegraaf A, Roeleveld RJ, Postmus PE, Heethaar RM, Van Rossum $A C$, and Boonstra A. Impaired left ventricular filling due to right ventricular pressure overload in primary pulmonary hypertension: Noninvasive monitoring using mri. Chest 119: 1761-1765, 2001.

30. Menzel T, Kramm T, Mohr-Kahaly S, Mayer E, Oelert H, and Meyer J. Assessment of cardiac performance using tei indices in patients undergoing pulmonary thromboendarterectomy. Ann Thorac Surg 73: 762-766, 2002.

31. Menzel T, Wagner S, Kramm T, Mohr-Kahaly S, Mayer E, Braeuninger S, and Meyer J. Pathophysiology of impaired right and left ventricular function in chronic embolic pulmonary hypertension: Changes after pulmonary thromboendarterectomy. Chest 118 : 897-903, 2000.

32. Moulopoulos SD, Sarcas A, Stamatelopoulos S, and Arealis E. Left ventricular performance during by-pass or distension of the right ventricle. Circ Res 17: 484-491, 1965.

33. Moustapha A, Kaushik V, Diaz S, Kang SH, and Barasch E. Echocardiographic evaluation of left-ventricular diastolic function in patients with chronic pulmonary hypertension. Cardiology 95: 96-100, 2001.

34. Patterson SW, and Starling EH. On the mechanical factors which determine the output of the ventricles. J Physiol 48: 357-379, 1914. 


\section{Chapter 6}

35. Reesink HJ, Marcus JT, Tulevski, II, Jamieson S, Kloek JJ, Vonk Noordegraaf A, and Bresser P. Reverse right ventricular remodeling after pulmonary endarterectomy in patients with chronic thromboembolic pulmonary hypertension: Utility of magnetic resonance imaging to demonstrate restoration of the right ventricle. J Thorac Cardiovasc Surg 133: 58-64, 2007.

36. Sadeghi HM, Kimura BJ, Raisinghani A, Blanchard DG, Mahmud E, Fedullo PF, Jamieson SW, and DeMaria AN. Does lowering pulmonary arterial pressure eliminate severe functional tricuspid regurgitation? Insights from pulmonary thromboendarterectomy. J Am Coll Cardiol 44: 126-132, 2004.

37. Schena M, Clini E, Errera D, and Quadri A. Echo-doppler evaluation of left ventricular impairment in chronic cor pulmonale. Chest 109: 1446-1451, 1996.

38. Stojnic BB, Brecker SJ, Xiao HB, Helmy SM, Mbaissouroum M, and Gibson DG. Left ventricular filling characteristics in pulmonary hypertension: A new mode of ventricular interaction. Br Heart J 68: 16-20, 1992.

39. Sztajzel J, Ruedin P, Monin C, Stoermann C, Leski M, Rutishauser W, and Lerch R. Effect of altered loading conditions during haemodialysis on left ventricular filling pattern. Eur Heart J 14: 655-661, 1993.

40. Taylor RR, Covell JW, Sonnenblick EH, and Ross J, Jr. Dependence of ventricular distensibility on filling of the opposite ventricle. Am J Physiol 213: 711-718, 1967.

41. Tei C, Dujardin KS, Hodge DO, Kyle RA, Tajik AJ, and Seward JB. Doppler index combining systolic and diastolic myocardial performance: Clinical value in cardiac amyloidosis. J Am Coll Cardiol 28: 658-664, 1996.

42. ter Keurs HE, Rijnsburger $\mathbf{W H}$, van Heuningen R, and Nagelsmit MJ. Tension development and sarcomere length in rat cardiac trabeculae. Evidence of lengthdependent activation. Circ Res 46: 703-714, 1980.

43. Thistlethwaite PA, Kaneko K, Madani MM, and Jamieson SW. Technique and outcomes of pulmonary endarterectomy surgery. Ann Thorac Cardiovasc Surg 14: 274-282, 2008.

44. Visner MS, Arentzen CE, Crumbley AJ, 3rd, Larson EV, O'Connor MJ, and Anderson RW. The effects of pressure-induced right ventricular hypertrophy on left ventricular diastolic properties and dynamic geometry in the conscious dog. Circulation 74: 410-419, 1986.

45. West JB, Dollery CT, and Naimark A. Distribution of blood flow in isolated lung; relation to vascular and alveolar pressures. J Appl Physiol 19: 713-724, 1964. 



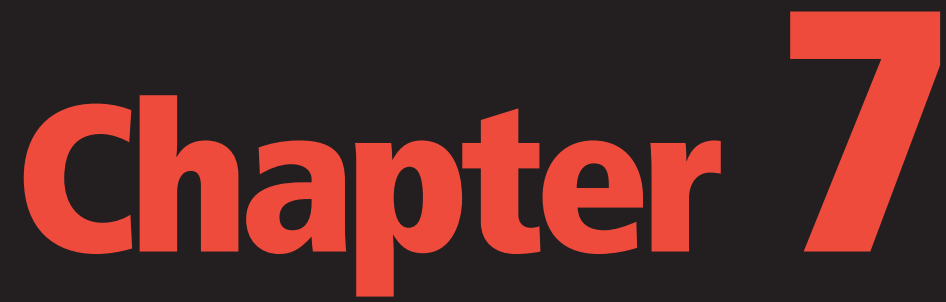

\section{Patient-Specific Modeling for Assessment of Ventricular Mechanical Asynchrony in Pulmonary Arterial Hypertension}

Departments of ${ }^{1}$ Physiology and ${ }^{2}$ Biomedical Engineering, Cardiovascular Research Institute Maastricht (CARIM), Maastricht University, Maastricht, The Netherlands. Departments of ${ }^{3}$ Physics and Medical Technology, ${ }^{4}$ Physiology, and ${ }^{5}$ Pulmonary Diseases, Institute for Cardiovascular Research (ICaR-VU), VU University Medical Center, Amsterdam, The Netherlands. 


\section{ABstract}

Pulmonary arterial hypertension (PAH) is associated with prolonged myofiber shortening in the right ventricular (RV) free wall (RVfw) compared to that in the left ventricular (LV) free wall and with early diastolic lengthening of the septum. It is unclear how, in PAH, mechanical overload of the RVfw leads to the observed ventricular mechanical asynchrony. In this study, we hypothesize that delay of mechanical relaxation of the RVfw alone can explain ventricular mechanical asynchrony in PAH. In $21 \mathrm{PAH}$ patients, aortic and pulmonary arterial pressures were obtained by arm-cuff measurements and right heart catheterization, respectively. Ventricular volume and circumferential strain data were obtained by magnetic resonance imaging. A mathematical model of ventricular mechanics and hemodynamics was fitted patient-specifically to available arterial pressure and ventricular volume data. Delay of RVfw peak shortening as predicted by the model $(0.120 \pm 0.048 s$; mean $\pm S D)$ was similar to the measured value $(0.112 \pm 0.034$ s). Early diastolic septal lengthening was systematically underestimated $(6.0 \pm 3.0 \%$ simulated vs. $10.1 \pm 6.0 \%$ measured). In a subgroup of 4 PAH patients with right bundle branch block, agreement between measured and simulated values of early diastolic septal lengthening improved further by imposing delayed onset of RVfw mechanical activation. In conclusion, we developed a patient-specific modeling approach that enables quantitative assessment of ventricular mechanical asynchrony in a hetero-geneous group of PAH patients. Patient-specific simulations showed that, in PAH, ventricular mechanical asynchrony is most likely caused by delay of mechanical relaxation of the RVfw either due to prolonged mechanical activation, delayed onset of activation, or both. 


\section{INTRODUCTION}

In the normal heart, synchronous activation and relaxation of the left ventricular (LV) free wall (LVfw), the interventricular septum (Sept), and the right ventricular (RV) free wall (RVfw) results in relatively uniform deformation of the ventricular walls during the cardiac cycle. ${ }^{20,27,29}$ In patients with pulmonary arterial hypertension (PAH) and chronic thromboembolic pulmonary hypertension (CTEPH), however, RV decompensation is associated with ventricular mechanical asynchrony as evidenced by prolonged duration of shortening and delayed peak shortening of the RVfw as compared to that of the LVfw and the ventricular septum. ${ }^{23,27}$ This ventricular mechanical asynchrony is related to disease severity and functional class of the patient. ${ }^{23}$ When left untreated, PAH leads to right heart failure and premature death. ${ }^{28,34}$ Several studies showed that morbidity and mortality of PAH patients are determined by the ability of the RV to maintain normal stroke volume and cardiac output., ${ }^{8,32}$ The mechanism by which pulmonary hypertension leads to ventricular mechanical asynchrony and right heart failure is poorly understood.

Several clinical and experimental studies showed that chronic pulmonary hypertension is associated with significant electrophysiological changes in the RVfw myocardium. $7,15,21,22$ In patients with CTEPH, action potential duration is increased and conduction velocity is decreased in the RVfW compared to those in the LVfw. ${ }^{15}$ Moreover, myocyte activation and repolarization are completed later in the RVfw than in the LVfw. ${ }^{7}$ In experimental animals ${ }^{16}$ as well as in patients ${ }^{17}$ with $\mathrm{PAH}$, profound abnormalities have been observed of the electrocardiogram (ECG) indicating decreased action potential duration heterogeneity and discordance of ventricular depolarization and repolarization. Despite all these important experimental and clinical observations, it is still unknown whether electrophysiological remodeling of the RVfw alone can explain the ventricular mechanical asynchrony in PAH and CTEPH.

In the present study, we aimed to elucidate the mechanism that underlies ventricular mechanical asynchrony in PAH and CTEPH. We assessed the hypothesis that ventricular mechanical asynchrony in patients with chronic pulmonary hypertension is caused by delay of mechanical relaxation of the RVfw. To test this hypothesis, we combined clinical measurements, as obtained in a group of PAH patients, with a novel patient-specific modeling approach. In 21 PAH patients, global hemodynamic status was assessed by measurement of left and right ventricular pressure and volume data using right heart catheterization and magnetic resonance (MR) imaging, respectively. ${ }^{27}$ Ventricular mechanical 
asynchrony was assessed by obtaining time courses of circumferential strain in the three ventricular walls by analysis of MR myocardial tagging images.

A numerical mode ${ }^{26}$ describing cardiovascular mechanics and hemodynamics including ventricular interaction was used to simulate the most likely hemodynamic status of each individual patient. In a first patient-specific simulation, the model was fitted to the ventricular volume and pressure data as obtained in each individual patient while assuming normal physiological behavior of the ventricular myocardium. Then, simulated strain patterns in the RVfw and Sept were compared with the measured strain patterns. In a second simulation step, a subgroup of four patients with coexisting right bundle branch block (RBBB) was further evaluated to test whether delayed onset of RVfw mechanical activation can more accurately explain the ventricular mechanical asynchrony as revealed by the ventricular strain patterns measured in those patients.

\section{MetHOdS}

\section{Patient data acquisition}

\section{Patient characteristics}

Twenty-one patients ( 5 men and 16 women, mean age $43 \pm 14$ years) with chronic pulmonary hypertension were included, i.e., 16 idiopathic PAH and 5 CTEPH. The patient group contained patients with New York Heart Association class II $(n=3)$, III $(n=17)$, and IV $(n=1)$. The medical ethical committee of the VU Medical Center approved the measurements used in the present study. More detailed descriptions of patient characteristics and of data acquisition procedures have been published previously. ${ }^{27}$ Relevant patient data are summarized in Table 7.1.

\section{Pressure data}

In all patients, pulmonary artery pressure was measured invasively by right heart catheterization. This pressure registration was used to calculate mean, systolic, and diastolic pulmonary artery pressure. Simultaneously, an arm-cuff blood pressure measurement was performed to obtain systolic and diastolic arterial blood pressure. Additionally, a standard 12-lead ECG was recorded. Based on ECG morphology (Table 7.1), a subgroup of four patients had an RBBB (3 incomplete and 1 complete). 


\section{Ventricular volume data}

LV and RV wall volumes and end-diastolic cavity volumes were calculated from a stack of short-axis cine magnetic resonance (MR) images covering both ventricles.

\section{Ventricular strain data}

An MR myocardial tagging procedure was applied with Complementary Spatial Modulation of Magnetization to acquire a time series of tagging images in the mid-ventricular short-axis plane. ${ }^{40,41}$ Midwall circumferential strain patterns in the LVfw, the Sept, and the RVfw were obtained by analyzing the MR tagging images with the Harmonic Phase procedure. ${ }^{30,40}$ Temporal resolution amounted to 14 ms. The ECG R-wave was used as reference point with zero strain. Heart rate $(H R)$ was obtained from the same ECG.

For quantitative analysis, the circumferential strain curves were filtered using a second-order Savitsky-Golay smoothing filter with a frame width of 9 data points. ${ }^{33}$ Figure 7.1 shows a typical example of filtered LVfw, Sept, and RVfw circumferential strain curves obtained in a PAH patient (Patient 15).

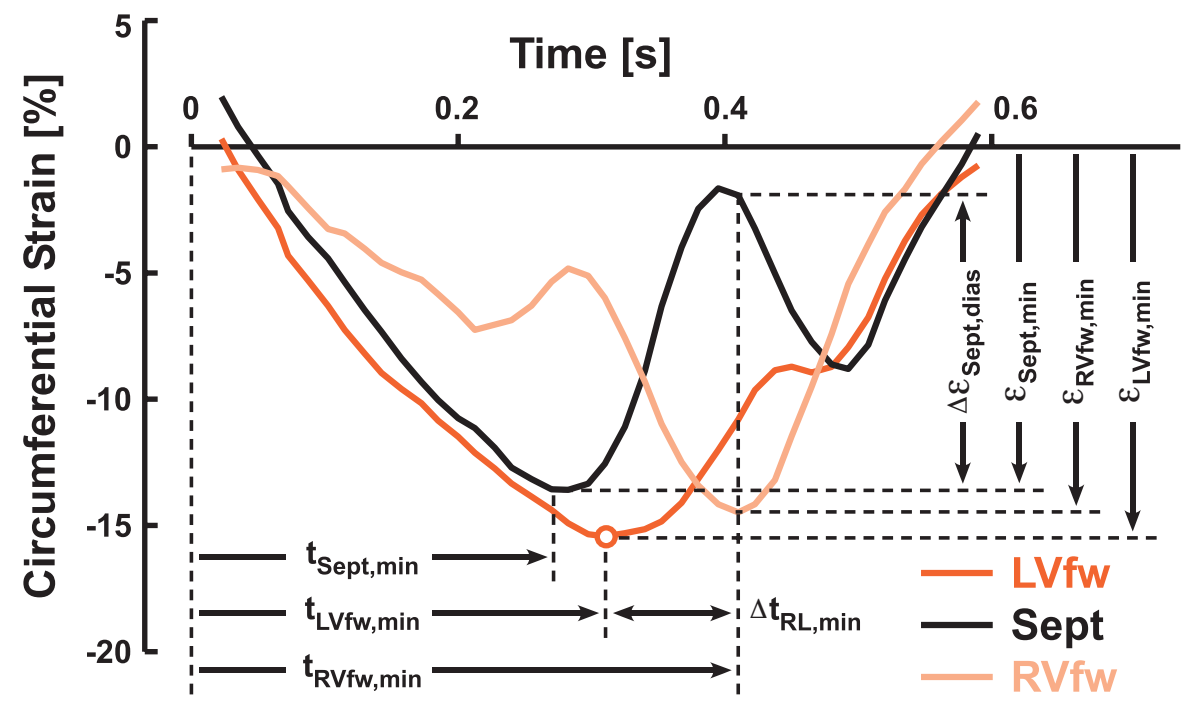

Figure 7.1: Time courses of circumferential strain as measured in the left ventricular free wall (LVfw), septum (Sept), and right ventricular free wall (RVfw) during a cardiac cycle in a pulmonary arterial hypertension (PAH) patient (Patient 15). Time zero corresponds to peak ECG R-wave serving as zero strain reference. Characteristic strains $(\varepsilon)$ : $\varepsilon_{\mathrm{LVfw} / \mathrm{RVfw} / \mathrm{Sept}, \mathrm{min}}=$ minimum strain in the LVfw/RVfw/Sept; $\Delta \varepsilon_{\text {Sept,dias }}=$ early diastolic septal lengthening. Characteristic times $(\mathrm{t}): \mathrm{t}_{\mathrm{LVfw} / \mathrm{RVfw} / \mathrm{Sept} \text {, } \min }=$ time to minimum strain in the LVfw/RVfw/Sept; $\Delta t_{R L, m i n}=R V f w-L V f w$ time delay of minimum strain. 
Minimum strain $\left(\varepsilon_{\min }\right)$ and time from ECG R-wave to minimum strain $\left(t_{\min }\right)$ were calculated for the LVfw, the Sept, and the RVfw (Figure 7.1). Ventricular mechanical asynchrony was quantified by the delay of the moment of minimum $\mathrm{RVfw}$ strain with respect to moment of minimum LVfw strain $\left(\Delta t_{\mathrm{RL}, \min }=\right.$ time to minimum RVfw strain - time to minimum LVfw strain) and by early diastolic septal lengthening $\left(\Delta \varepsilon_{\text {sept,dias }}=\right.$ Sept strain at moment of minimum RVfw strain minimum Sept strain).

\section{Patient-specific simulations}

\section{Model design}

For the present study, we used a numerical model that simulates ventricular mechanics and hemodynamics realistically under normal and pulmonary hypertensive conditions. ${ }^{25,26}$ This model consisted of a module (TriSeg model ${ }^{26}$ ) describing ventricular mechanics, including mechanical interaction of the ventricular walls, embodied in the CircAdapt model describing mechanics of the whole human circulation. ${ }^{1}$

In the TriSeg model, three thick-walled spherical segments representing the ventricular walls join in a common junction so that they encapsulate the LV and RV cavity. Given LV and RV cavity volume, area and curvature of the LVfw and RVfw are calculated while assuming an initial estimate of Sept geometry. From wall geometry, representative myofiber strain is calculated for each wall. From myofiber strain, myofiber stress is determined using constitutive equations describing sarcomere mechanics. The latter modele ${ }^{26}$ incorporates experimental findings on isolated cardiac muscle, ${ }^{9,36}$ i.e., velocity of sarcomere shortening increases with passive stretch, strength of activation increases with sarcomere length, and duration of activation increases with sarcomere length. Using myofiber stress and wall geometry, total radial and axial force components of tension acting on the junction are calculated. Then, the initially estimated septal geometry is adjusted so that equilibrium of forces is satisfied in the junction. As a result, ventricular cavity volumes and pressures as well as geometries of the three ventricular walls are known, together with their myofiber stress and strain.

The CircAdapt model of the whole circulation ${ }^{1}$ provides the required hemodynamic environment for the ventricles as represented by the TriSeg model. The CircAdapt model is composed of modules representing atrial walls, cardiac valves, large blood vessels, and peripheral resistances. Furthermore, the model allows adaptation of size and mass of cardiac walls and large blood vessels to normalize local mechanical load to tissue-specific physiological levels. ${ }^{1,26}$ The combined CircAdapt/TriSeg model allows beat-to-beat simulation of time-dependent volumes 
and pressures of the cardiac cavities, geometries and myofiber mechanics of the cardiac walls, and flows through valves.

In the present study, we developed a patient-specific simulation procedure that enables fitting of the model to measurements as obtained in individual PAH patients.

\section{Simulation of the average PAH patient}

The model was initialized by simulation of human cardiovascular mechanics and hemodynamics under normal ventricular loading conditions at rest. The methods used to obtain this normal simulation have been published in detail elsewhere. ${ }^{26}$ Next, pulmonary vascular resistance was increased so that mean pulmonary artery pressure (mPAP) was equal to $25 \mathrm{mmHg}$. The heart and large blood vessels were fully compensated by adaptation to this level of increased mechanical load. This value of MPAP was chosen because pulmonary hypertension is clinically defined as mPAP > $25 \mathrm{mmHg}$ at rest. ${ }^{11}$

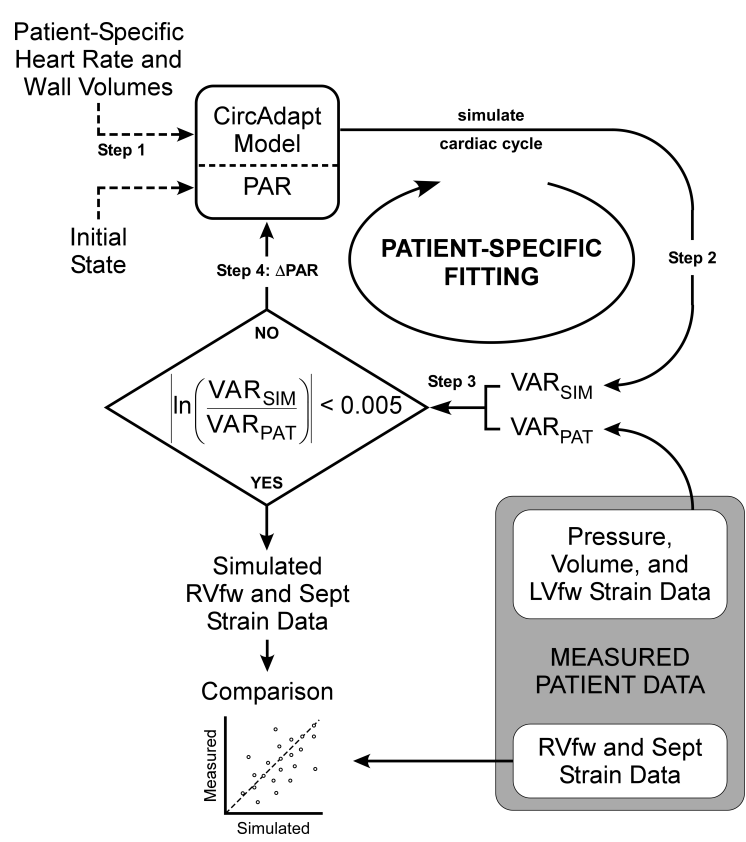

Figure 7.2: Schematic representation of the patient-specific simulation procedure. Starting from an initial state, a cardiac cycle was simulated with patientspecific settings of heart rate and ventricular wall volumes. Simulated parameter values $V A R_{\text {SIM }}$ are derived from the simulation in the same way as their measured equivalents $V_{A} R_{\text {PAT }}$ are derived from the strain patterns measured in the patients. Differences between VAR $R_{\text {PAT }}$ and $V A R_{\text {SIM }}$ are used to improve the fit iteratively by adjusting model parameters PAR. If the difference is below a threshold, iteration stops. Finally, simulated and measured values of characteristic RVfw and Sept strain parameters were compared. 
Table 7.1 Measured input parameters (VARPAT) obtained in 21 pulmonary arterial hypertension patients $^{\dagger}$

\begin{tabular}{|c|c|c|c|c|c|c|c|c|c|c|c|c|c|}
\hline \multirow[t]{2}{*}{$\begin{array}{c}\text { Patient } \\
\#\end{array}$} & \multirow[t]{2}{*}{$\begin{array}{c}\text { HR } \\
\text { [bpm] }\end{array}$} & \multicolumn{2}{|c|}{$\begin{array}{l}\text { Wall Volume } \\
\text { [ml] }\end{array}$} & \multicolumn{2}{|c|}{$\begin{array}{l}\text { EDV } \\
{[\mathrm{ml}]}\end{array}$} & \multicolumn{2}{|c|}{$\begin{array}{c}\mathrm{BP} \\
{[\mathrm{mmHg}]}\end{array}$} & \multicolumn{3}{|c|}{$\begin{array}{c}\text { PAP } \\
{[\mathrm{mmHg}]}\end{array}$} & \multirow[t]{2}{*}{$\begin{array}{c}\varepsilon_{\text {LVfw,min }} \\
{[\%]}\end{array}$} & \multirow[t]{2}{*}{$\begin{array}{l}\mathrm{t}_{\mathrm{LVfw}, \mathrm{min}} \\
{[\mathrm{ms}]}\end{array}$} & \multirow[t]{2}{*}{ Etiology } \\
\hline & & LV & RV & LV & RV & Sys & Dias & Sys & Dias & Mean & & & \\
\hline 1 & 78 & 112 & 79 & 101 & 157 & 100 & 50 & 101 & 35 & 62 & -14.1 & 285 & IPAH \\
\hline 2 & 67 & 130 & 259 & 126 & 554 & 110 & 80 & 71 & 28 & 47 & -7.0 & 270 & CTEPH* \\
\hline 3 & 71 & 82 & 57 & 133 & 175 & 120 & 90 & 84 & 31 & 49 & -21.2 & 315 & IPAH \\
\hline 4 & 61 & 112 & 65 & 71 & 227 & 110 & 75 & 93 & 34 & 54 & -13.8 & 298 & IPAH \\
\hline 5 & 81 & 155 & 41 & 82 & 146 & 110 & 50 & 102 & 34 & 61 & -14.5 & 270 & IPAH \\
\hline 6 & 74 & 103 & 104 & 131 & 209 & NA & NA & 83 & 33 & 51 & -14.1 & 280 & $\mathrm{CTEPH}^{* *}$ \\
\hline 7 & 86 & 116 & 63 & 135 & 176 & 120 & 70 & 97 & 32 & 57 & -17 & 288 & PAH $^{* *}$ \\
\hline 8 & 57 & 84 & 43 & 169 & 193 & 115 & 65 & 41 & 15 & 28 & -15 & 358 & IPAH \\
\hline 9 & 88 & 85 & 100 & 65 & 181 & 110 & 80 & 109 & 38 & 64 & -11. & 258 & IPAH \\
\hline 10 & 102 & 83 & 81 & 106 & 219 & 120 & 80 & 77 & 40 & 53 & -15.1 & 228 & $\mathrm{PAH}^{* *}$ \\
\hline 11 & 105 & 71 & 73 & 75 & 176 & 115 & 72 & 112 & 37 & 70 & -13 & 213 & IPAH \\
\hline 12 & 85 & 112 & 68 & 120 & 139 & 120 & 80 & 64 & 21 & 50 & & & CTEPH \\
\hline 13 & 103 & 75 & 59 & 76 & 176 & NA & NA & 87 & 38 & 60 & -13 & 29 & IPAH \\
\hline 14 & 75 & 82 & 106 & 92 & 247 & 90 & 70 & 89 & 39 & 58 & -10 & 31 & IPAH \\
\hline 15 & 80 & 112 & 61 & 103 & 146 & 120 & 80 & 88 & 29 & 52 & -15.4 & 310 & IPAH \\
\hline 16 & 80 & 155 & 153 & 129 & 285 & 122 & 67 & 130 & 57 & 90 & -12.4 & 285 & IPAH \\
\hline 17 & 57 & 103 & 76 & 82 & 213 & 135 & 80 & 98 & 35 & 56 & -14.4 & 398 & IPAH \\
\hline 18 & 92 & 87 & 64 & 82 & 202 & 100 & 70 & 92 & 33 & 58 & -15 & 228 & CTEPH \\
\hline 19 & 70 & 97 & 90 & 101 & 294 & NA & NA & 89 & 34 & 55 & -15.3 & $\angle 30$ & CTEPH \\
\hline 20 & 80 & 85 & 41 & 137 & 169 & 100 & 60 & 49 & 11 & 32 & -18.4 & 252 & IPAH \\
\hline 21 & 98 & 91 & 74 & 95 & 265 & 105 & 65 & 79 & 34 & 50 & -18.0 & 275 & IPAH \\
\hline ean & 80 & 102 & 84 & 105 & 217 & 112 & 71 & 87 & 33 & 55 & -14.9 & 286 & \\
\hline 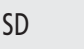 & 14 & 24 & 48 & 28 & 89 & 11 & 11 & 20 & 9 & 13 & 3.5 & 43 & \\
\hline
\end{tabular}

For each measurement, mean value and standard deviation (SD) are presented at the bottom of the table.

† Data obtained from Marcus et al. ${ }^{27}$

* Patient 2 had a complete right bundle branch block.

**Patients 6, 7, and 10 had an incomplete right bundle branch block.

$\mathrm{BP}=$ brachial arterial blood pressure; $\mathrm{CTEPH}=$ chronic thromboembolic pulmonary hypertension; Dias = diastolic; $E D V=$ end-diastolic volume; $\mathrm{HR}=$ heart rate; $\mathrm{IPAH}=$ idiopathic pulmonary arterial hypertension; LV = left ventricular; NA = not available; PAP = pulmonary artery pressure; $\mathrm{RV}=$ right ventricular; Sys = systolic; $\mathrm{t}_{\mathrm{LVfw}, \min }=$ time to minimum strain; $\varepsilon_{\mathrm{LVfw}, \text { min }}$ $=$ minimum strain in the LV free wall. 
Using the resulting simulation as initial state, the average patient was simulated by fitting the model to the average values of the measurements given in Table 7.1. Below, the used fitting algorithm is described in steps (Figure 7.2).

Step 1: HR and ventricular wall volumes were set to average values measured in the patient group (Table 7.1). LVfw and Sept wall volumes were set to $2 / 3$ and $1 / 3$ of total LV wall volume, respectively, which fractions are normal for PAH patients. $5,10,18,31$ 


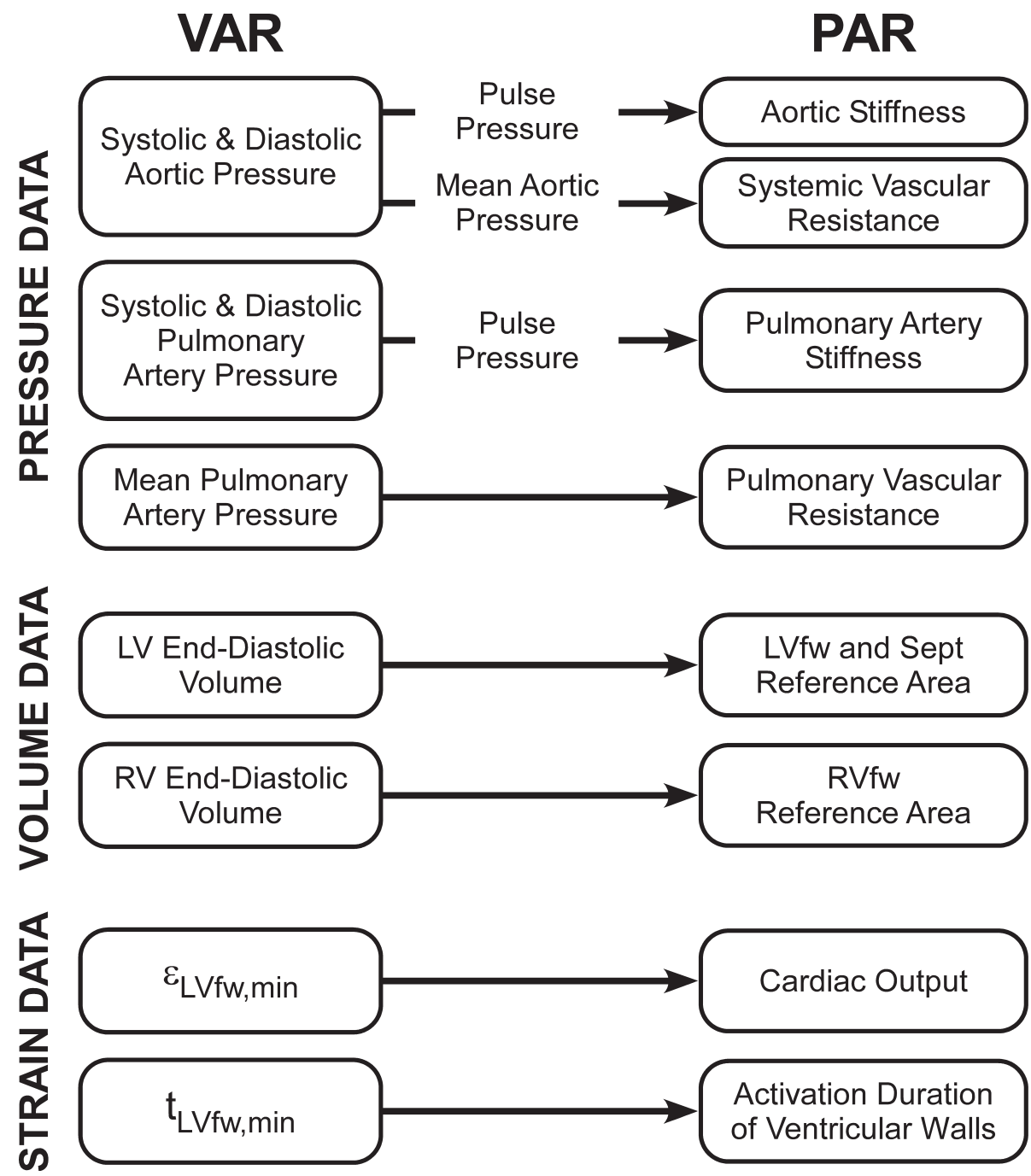

Figure 7.3: Relations between input parameters (VAR) and adjustable model parameters (PAR) as used for patient-specific fitting. Differences between measured and simulated values of ventricular pressure and volume data and of LVfw circumferential strain were used to adjust cardiac output and other model parameters representing mechanical properties of pulmonary and systemic circulations and of ventricular walls. Abbreviations: SBP/DBP = systolic/diastolic aortic blood pressure; sPAP/dPAP/mPAP = systolic/diastolic/mean pulmonary artery pressure; EDV = end-diastolic volume; LV = left ventricular; RV = right ventricular; Other abbreviations and symbols as in Figure 7.1 . 
Then, in an iterative fitting procedure, a set of heuristically chosen model parameters (VAR in Figures 7.2 and 7.3) was adjusted so that simulated values of a set of variables (VAR in Figures 7.2 and 7.3) were equal to the corresponding average values as measured in the patient group (Table 7.1).

Step 2: A single cardiac cycle was simulated. All variables VAR were derived from the simulated time courses of arterial pressures, ventricular volumes, and LVfw strain.

Step 3: For each variable VAR, a scaling factor was quantified as the ratio of simulated to measured value $\left(V A R_{\text {SIM }} / V A R_{\text {PAT }}\right)$.

Step 4: The scaling factors were used to adjust model parameters PAR (Figure 7.3). Pulmonary arterial pulse pressure (systolic-diastolic pressure difference) was used to adjust pulmonary artery stiffness. Mean pulmonary artery pressure was used to adjust pulmonary vascular resistance. Analogously, aortic pulse pressure and mean aortic pressure were used to adjust aortic stiffness and systemic vascular resistance, respectively. Mean aortic pressure was estimated by the geometric mean of SBP and DBP. ${ }^{6}$ Cardiac output was adjusted so that minimum $\mathrm{LVfw}$ strain ( $\varepsilon_{\mathrm{LVfw}, \min }$ in Figure 7.1) was equal to its measured value. Durations of mechanical activation of the three ventricular walls were adjusted proportionally so that simulated time to minimum LVfw strain ( $\mathrm{t}_{\mathrm{LVfw} w \text { min }}$ in Figure 7.1 ) was equal to its measured value. RV and LV end-diastolic volumes were used to adjust RVfw reference area and summed LVfw and Sept reference area, respectively. In the model, reference area of a wall is defined as the midwall surface area at reference sarcomere length of $2.0 \mu \mathrm{m} .{ }^{26}$

The iterative fitting cycle (Steps 2-4) was repeated until VAR $R_{\text {SIM }}$ matched $V_{A A R}$ PAT with less than $0.5 \%$ error. After about $50-100$ cycles, a steady state was achieved representing the circulation of the average PAH patient.

\section{Simulation of the specific PAH patient}

For each set of patient-specific measurements given in Table 7.1, the abovementioned fitting procedure was applied using the average PAH patient as initial state (Figure 7.2). Finally, this resulted in 21 patient-specific simulations.

\section{Additional simulation of RBBB and tricuspid regurgitation}

To quantify the effect of delay of RVfw relaxation on ventricular strain patterns, a subgroup of four PAH patients (Patient 2, 6, 7, and 10) with coexisting RBBB was further evaluated. Starting from the thus far found patient-specific fits, onset of mechanical activation of the RVfw was delayed with $30 \mathrm{~ms}$ and $60 \mathrm{~ms}$ 
as compared to that of the LVfw and the Sept. Furthermore, there was clinical evidence of significant tricuspid regurgitation (TR) in Patient 2, i.e., RV stroke volume $>2 x$ LV stroke volume. In this patient, the effect of TR on ventricular strain patterns was investigated by setting leakage orifice area to $10 \%$ and $20 \%$ of tricuspid valve opening area.

\section{Comparison of simulated and measured strains}

For each patient-specific simulation, circumferential strains in the three ventricular walls were quantified relative to the status at moment of aortic valve opening. Linear regression and the Bland-Altman method were used to test similarity of six measured and simulated strain and timing variables, i.e., minimum Sept and RVfw strain, time to minimum Sept and RVfw strain, delay of RVfw minimum strain with respect to LVfw minimum strain, and early diastolic septal lengthening (Figure 7.1). Analogously, measured strain curves of the subgroup of patients with RBBB were compared with the strain curves obtained from the simulations with and without additional simulation of RBBB.

\section{Sensitivity analysis}

A sensitivity analysis (Appendix 7.A) was performed to quantify relative sensitivities of $V A R_{\text {SIM }}$ to changes in model parameters PAR.

\section{RESULTS}

\section{Patient-specific simulations}

In Table 7.2, group mean values of strain and timing parameters are presented, as derived from the measured strain curves and as obtained from the patientspecific simulations. Patient group heterogeneity appeared quite large as reflected by high standard deviations of the measured pressures, volumes, and strains (Table 7.1 and 7.2). Despite the large heterogeneity, patient-specific simulation resulted in steady state solutions in all patients. Furthermore, average values and standard deviations of the differences between measured and simulated parameter values are shown in Table 7.2. The small mean differences between measured and simulated timing parameters suggest that the patientspecific simulation procedure did not result in systematic errors in timing of ventricular strains. Differences between measured and simulated strain parameters, however, show that RVfw peak shortening $\left(\varepsilon_{\text {RVfw,min }}\right)$ and early diastolic septal lengthening $\left(\Delta \varepsilon_{\text {Sept,dias }}\right)$ were systematically underestimated in our simulations. Moreover, the large standard deviations (about $50 \%$ of mean value 
of measurements) of the differences of $\Delta \mathrm{t}_{\mathrm{RL}, \mathrm{min}}$ and of $\Delta \varepsilon_{\text {Sept,dias }}$ show that the used patient-specific fitting procedure does not accurately predict ventricular mechanical asynchrony in this patient group.
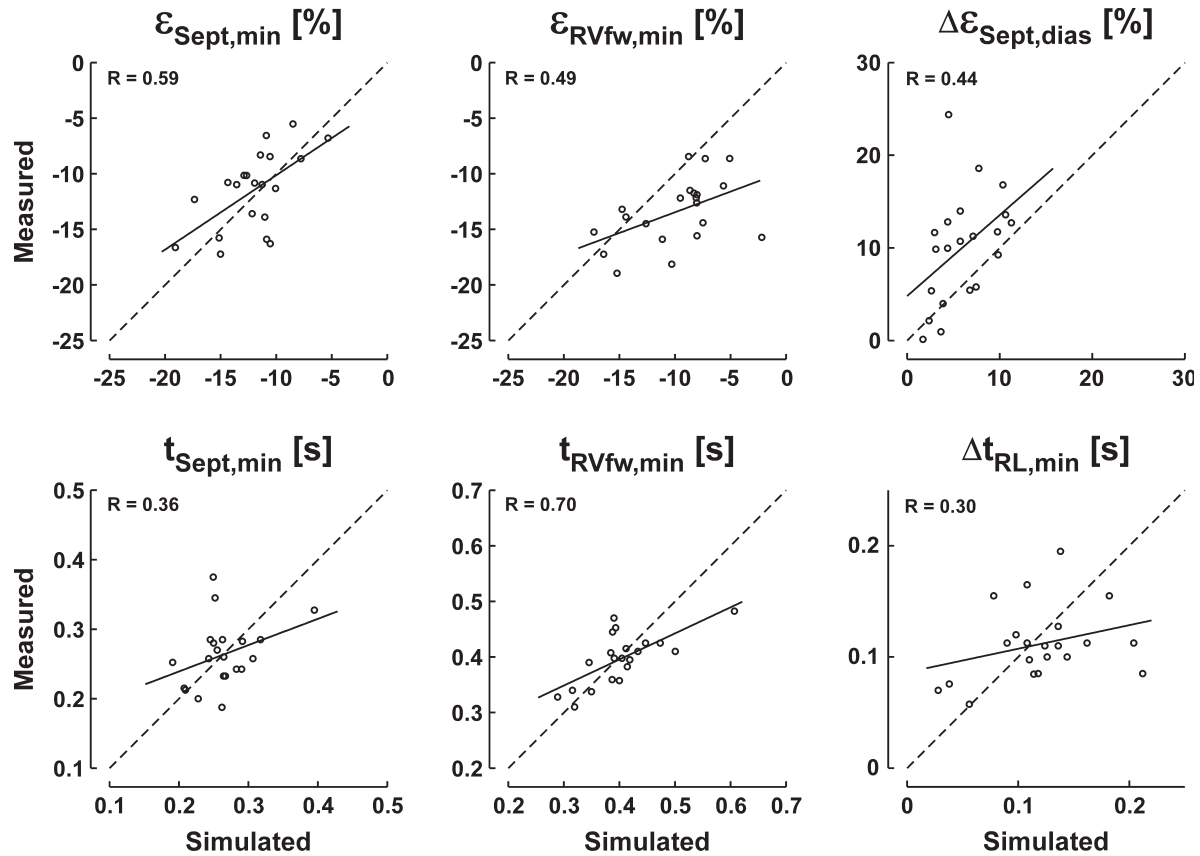

Figure 7.4: Measured vs. simulated values of characteristic strain and timing parameters as derived from strain measurements and patient-specific simulations in $21 \mathrm{PAH}$ patients. Linear regression line (solid line) and correlation coefficient ( $R$ ) are shown, as well as the line of identity (dashed line). Abbreviations as in Figure 7.1.

In Figure 7.4, measured values of strain and timing parameters of all patients are plotted as function of their corresponding simulated values. Regression analysis revealed significant correlations $(p<0.05)$ between measured and simulated values for all three strain parameters (top row of Figure 7.4) and for time to minimum RVfw strain. Although the absolute value of early diastolic septal lengthening was underestimated, the patient-specific fitting procedure predicted ventricular mechanical asynchrony (positive $\Delta t_{R L, \text { min }}$ and $\Delta \varepsilon_{\text {Sept,dias }}$ ) in all patients. Full time courses of measured and simulated strains of all patients are shown in Appendix 7.B. 
Table 7.2 Measured vs. simulated values of strain parameters: simulations with physiological mechanical properties of the myocardial tissue

\begin{tabular}{|c|c|c|c|}
\hline & $\begin{array}{l}\text { Measurements } \\
\text { (Meas) }\end{array}$ & $\begin{array}{l}\text { Simulations } \\
\text { (Sim) }\end{array}$ & $\begin{array}{c}\text { Difference } \\
\text { (Meas - Sim) }\end{array}$ \\
\hline & $n=21$ & $n=21$ & $n=21$ \\
\hline \multicolumn{4}{|c|}{ Strain parameters } \\
\hline$\varepsilon_{\text {Sept,min }}(\%)$ & $-11.5 \pm 3.5$ & $-12.0 \pm 3.1$ & $0.5 \pm 3.0$ \\
\hline$\varepsilon_{\mathrm{RVfw}, \min }(\%)$ & $-13.4 \pm 3.0$ & $-9.9 \pm 3.9$ & $-3.5 \pm 3.6$ \\
\hline$\Delta \varepsilon_{\text {Sept,dias }}(\%)$ & $10.1 \pm 6.0$ & $6.0 \pm 3.0$ & $4.1 \pm 5.4$ \\
\hline \multicolumn{4}{|c|}{ Timing parameters } \\
\hline $\mathrm{t}_{\text {Sept, min }}(\mathrm{s})$ & $0.263 \pm 0.046$ & $0.263 \pm 0.044$ & $0.000 \pm 0.051$ \\
\hline$t_{R V f w \text { min }}(s)$ & $0.397 \pm 0.046$ & $0.403 \pm 0.069$ & $-0.006 \pm 0.049$ \\
\hline$\Delta t_{R L \text { min }}(\mathrm{s})$ & $0.112 \pm 0.034$ & $0.120 \pm 0.048$ & $-0.008 \pm 0.049$ \\
\hline
\end{tabular}

Data are presented as mean value \pm standard deviation.

$t_{\mathrm{RVfw}, \text { min }}=$ time to minimum RVfW strain; $\mathrm{t}_{\mathrm{Sept,min}}=$ time to minimum Sept strain; $\varepsilon_{\mathrm{RVfw}, \text { min }}=$ minimum RVfw strain; $\varepsilon_{\text {Sept,min }}=$ minimum Sept strain; $\Delta t_{R L, m i n}=R V f w-L V f w$ time delay of minimum strain; $\Delta \varepsilon_{\text {Sept,dias }}=$ early diastolic septal lengthening.

\section{Additional simulation of RBBB and tricuspid regurgitation}

For the PAH patients with RBBB, time courses of measured ventricular strains are compared with those of simulated strains as obtained from the patient-specific simulations with and without added delay of RVfw mechanical activation (Figure 7.5). With $30 \mathrm{~ms}$ and $60 \mathrm{~ms}$ delay of RVfw mechanical activation, early diastolic septal lengthening $\left(\Delta \varepsilon_{\text {Sept,dias }}\right)$ increased by $4.4 \pm 0.7 \%$ and $9.5 \pm 1.3 \%$, respectively. Delay of minimum RVfw strain $\left(\Delta \mathrm{t}_{\mathrm{RL}, \mathrm{min}}\right)$ increased by $11 \pm 13 \mathrm{~ms}$ and $26 \pm 13 \mathrm{~ms}$, respectively. Moreover, the amplitude of RVfw shortening $\left(\varepsilon_{\mathrm{RVfw}, \mathrm{min}}\right)$ increased by $2.1 \pm 0.7 \%$ and $3.6 \pm 1.2 \%$, respectively. In Patients 6,7 , and 10 , best fits of simulated RVfw and Sept strain curves were obtained with $60 \mathrm{~ms}, 30$ $\mathrm{ms}$, and $60 \mathrm{~ms}$ delay of RVfw activation, respectively. In Patient 2, however, delay of RVfw activation duration alone (top rows of Figures 7.5 and 7.6) did not explain the measured high amplitude of RVfw shortening. Simulation of TR alone (left column of Figure 7.6), improved the fit of $\varepsilon_{\text {RVfw, }}$ min but decreased $\Delta \varepsilon_{\text {sept,dias. }}$. However, adding effects of both RBBB and TR to the simulation resulted in improved fits of $\varepsilon_{\mathrm{RVfw}, \min }$ as well as $\Delta \varepsilon_{\text {Sept,dias }}$ (right lower corner of Figure 7.6). 
Simulation

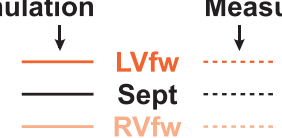

Patient 2

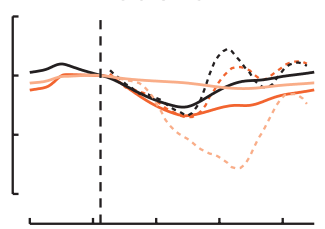

Patient 6

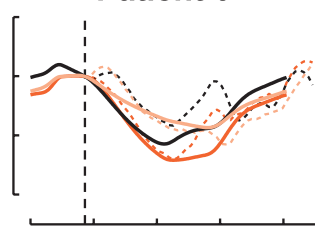

Patient 7

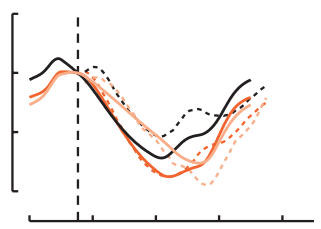

Patient 10

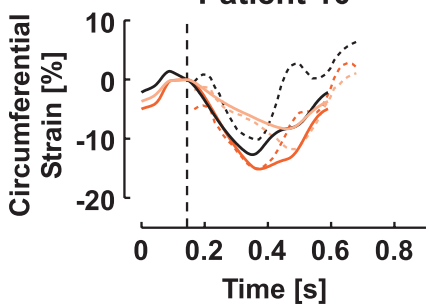

Additional Simulation of RBBB

30 ms Delay of RVfw Activation
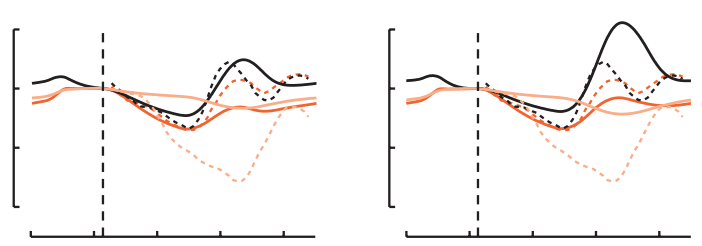

$60 \mathrm{~ms}$ Delay of RVfw Activation
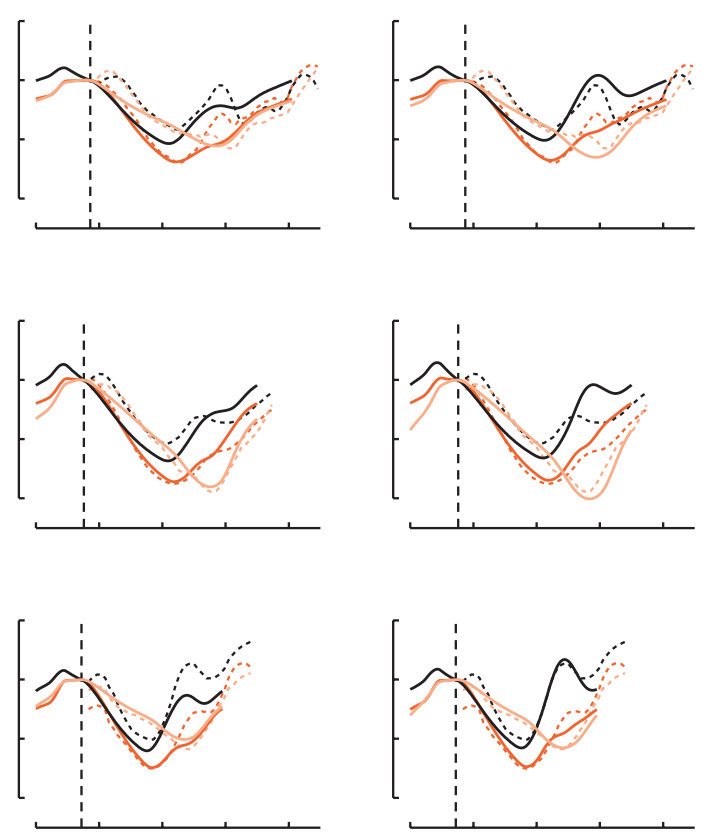

Figure 7.5: Time courses of measured (dashed lines) and simulated (solid lines) circumferential strains in the ventricular walls during a cardiac cycle of $4 \mathrm{PAH}$ patients with right bundle branch block (RBBB). Left column: measured strains are compared with strain curves as obtained by regular patient-specific simulation. Middle and right column: patient-specific simulation with additional effect of RBBB. Timing of zero strain references are indicated by vertical dashed lines. For the measurements, this reference corresponds to the ECG R-wave. For the simulations, this reference corresponds to the moment of aortic valve opening. 
Additional Simulation of RBBB

Patient 2

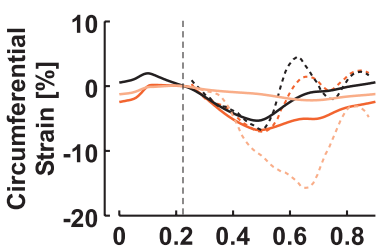

Time $[\mathrm{s}]$

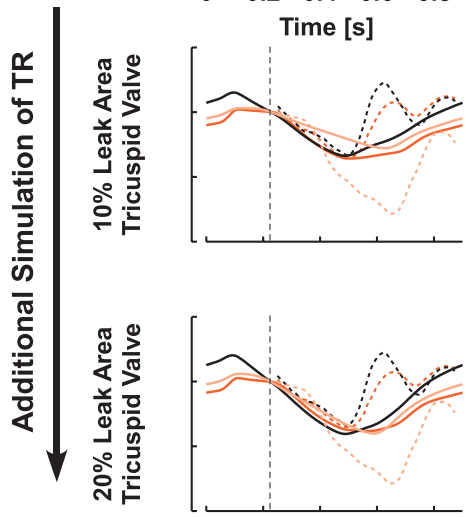

30 ms Delay of RVfw Activation
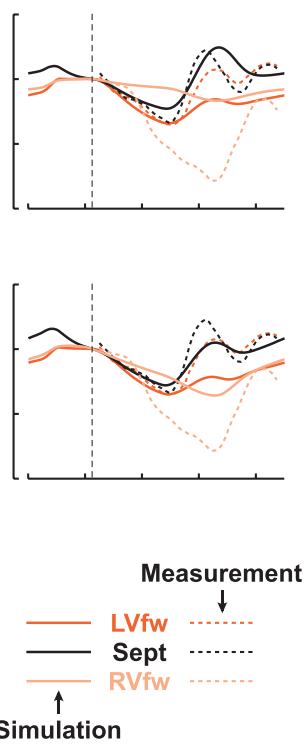

$60 \mathrm{~ms}$ Delay of RVfw Activation
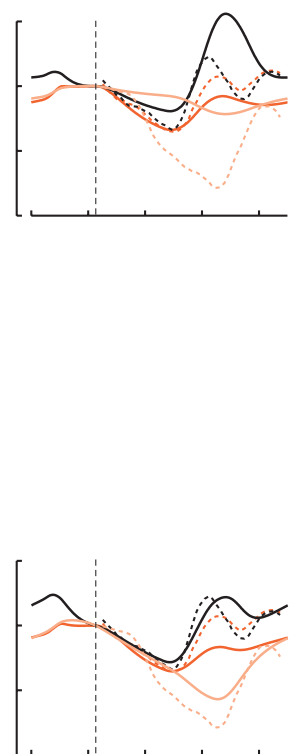

Figure 7.6: Time courses of measured (dashed lines) and simulated (solid lines) circumferential strains in the ventricular walls during a cardiac cycle of Patient 2 with chronic thromboembolic pulmonary hypertension, right bundle branch block (RBBB) and tricuspid regurgitation (TR). Upper left corner: measured strains are compared with strain curves as obtained by regular patientspecific simulation. From upper left corner to upper right corner: patient-specific simulation with additional effect of RBBB. From upper left corner to lower left corner: patient-specific simulation with additional simulation of TR. From upper left corner to lower right corner: patient-specific simulation with additional simulation of both RBBB and TR. In Patient 2, best overall fit was achieved by additional simulation of both RBBB and TR (lower right corner). Timing of zero strain references are indicated by vertical dashed lines. For the measurements, this reference corresponds to the ECG R-wave. For the simulations, this reference corresponds to the moment of aortic valve opening.

For the subgroup of PAH patients with RBBB, Figure 7.7 shows the improvement of the patient-specific fits as a result of additional simulation of RBBB (and TR in Patient 2). For all six characteristic strain parameters, all individual data points moved toward the line of identity when simulating 
additional RBBB (and TR in Patient 2). Both, mean and standard deviation of the difference decreased considerably for $\varepsilon_{R V f w, m i n}, \Delta \varepsilon_{\text {Sept,dias, }} t_{R V f w, m i n}$, and $\Delta t_{R L \text {,min }}$ (Table 7.3). Additional simulation of RBBB and TR did hardly affect absolute value and timing of Sept peak shortening, i.e., $\varepsilon_{\text {Sept,min }}$ and $t_{\text {Sept,min, }}$ respectively. No statistical analysis was performed in this subgroup due to the limited number of patients.
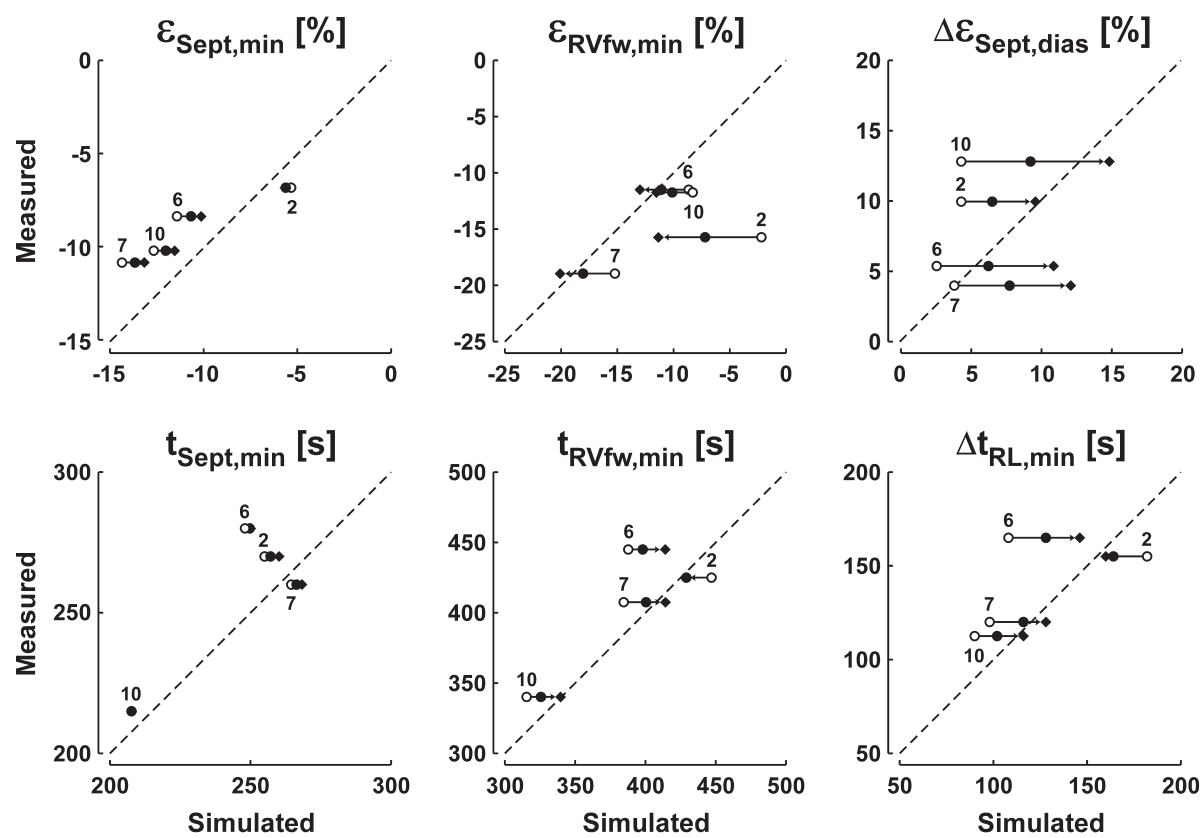

Figure 7.7: Measured vs. simulated values of characteristic strain and timing parameters as derived from strain measurements and patient-specific simulations of 4 PAH patients with RBBB. Open circles $(\bigcirc)$ indicate data points obtained from the regular patient-specific simulations (also shown in Figure 7.4). Closed circles $(\bullet)$ and diamonds $(\bullet)$ indicate data points obtained from patient-specific simulations with 30 ms and 60 ms delay of RVfw mechanical activation, respectively. Note that for Patient 2, not only delay of RVfw activation but also TR has been imposed by $10 \%$ and $20 \%$ leak area of the tricuspid valve. Numbers and arrows indicate patient number and direction of change of simulated parameter value, respectively. Dashed line represents line of identity. Abbreviations as in Figures 7.1 and 7.6 . 
Table 7.3 Differences between measured and simulated values of strain parameters in four patients with PAH and RBBB: effect of additional simulation of RBBB (and TR in Patient 2)

\begin{tabular}{|c|c|c|c|}
\hline & $\begin{array}{c}\text { Difference } \\
\text { (Meas - Sim) }\end{array}$ & $\begin{array}{c}\text { Difference } \\
\text { (Meas - Sim) }\end{array}$ & $\begin{array}{c}\text { Difference } \\
\text { (Meas - Sim) }\end{array}$ \\
\hline \multirow[t]{2}{*}{$\begin{array}{l}\text { Additional } \\
\text { simulation }\end{array}$} & No & $\begin{array}{c}\text { 30ms Delay of RVfw Activation } \\
(10 \% \text { TR in Patient 2) }\end{array}$ & $\begin{array}{c}\text { 60ms Delay of RVfw Activation } \\
\text { (20\% TR in Patient 2) }\end{array}$ \\
\hline & $n=4$ & $n=4$ & $n=4$ \\
\hline \multicolumn{4}{|l|}{ Strain parameters } \\
\hline$\varepsilon_{\text {Sept,min }}(\%)$ & $1.9 \pm 2.3$ & $1.5 \pm 1.8$ & $1.1 \pm 1.6$ \\
\hline$\varepsilon_{\mathrm{RVfw}, \min }(\%)$ & $-5.9 \pm 5.1$ & $-2.9 \pm 3.8$ & $-0.5 \pm 2.7$ \\
\hline$\Delta \varepsilon_{\text {sept,dias }}(\%)$ & $4.2 \pm 3.6$ & $0.5 \pm 3.6$ & $-3.9 \pm 3.7$ \\
\hline \multicolumn{4}{|l|}{ Timing parameters } \\
\hline$t_{\text {Sept, min }}(s)$ & $0.012 \pm 0.015$ & $0.011 \pm 0.016$ & $0.010 \pm 0.016$ \\
\hline$t_{\text {RVfw,min }}(s)$ & $0.021 \pm 0.033$ & $0.016 \pm 0.022$ & $0.005 \pm 0.017$ \\
\hline$\Delta \mathrm{t}_{\mathrm{RL}, \min }(\mathrm{s})$ & $0.019 \pm 0.035$ & $0.011 \pm 0.019$ & $0.001 \pm 0.012$ \\
\hline
\end{tabular}

Data are presented as mean difference \pm standard deviation of the difference.

$\mathrm{RBBB}=$ right bundle branch block; $\mathrm{TR}=$ tricuspid regurgitation; Other abbreviations as in Table 7.2.

\section{Discussion}

We developed a novel patient-specific fitting procedure to assess ventricular mechanics in 21 patients with PAH. In all patients, measured as well as patientspecifically simulated strain patterns showed early diastolic septal lengthening and delayed peak shortening of the RVfw as compared to that of the LVfw. These parameters of ventricular mechanical asynchrony were systematically underestimated in the patient-specific simulations. In a subgroup of four patients with PAH and RBBB, quantitative agreement between measured and simulated values of early diastolic septal lengthening and delay of RVfw peak shortening improved by delaying onset of RVfw mechanical activation. One of the latter four patients also had significant TR. In this patient, simulation of tricuspid valve leakage in addition to RBBB increased RVfw peak shortening and, thereby, further improved agreement between the simulated and the measured strain patterns.

In the present study, it was found that delay of mechanical relaxation of the RVfw alone predominantly caused ventricular mechanical asynchrony in the ventricular walls of PAH patients. Moreover, it has been shown that this 
happened irrespective whether the delay of RVfw relaxation is caused by prolonged RVfw mechanical activation, delayed onset of RVfw activation, or both. This is in agreement with measurements obtained by Hardziyenka et al. among CTEPH patients. ${ }^{15}$ In these patients, ventricular mechanical asynchrony was related to significant action potential prolongation and conduction slowing in the RVfw as compared to the LVfw. In a subgroup of CTEPH patients with RBBB, they found more pronounced delay of RVfw mechanical relaxation. Other studies showed that the ventricular mechanical asynchrony as a result of delayed mechanical RVfw relaxation may be a target for therapeutic intervention. ${ }^{14,25}$ These studies showed that early pacing of the RVfw may acutely reduce ventricular mechanical asynchrony and, thereby, improve RV pump function.

In all patients, patient-specific fitting resulted in delay of RVfw minimum strain with respect to LVfw minimum strain $\left(\Delta t_{R L, m i n}\right.$ in Figure 7.4$)$. Mean difference between measured and simulated $\Delta \mathrm{t}_{\mathrm{RL}, \mathrm{min}}$ was close to zero (Table 7.2), but the standard deviation of the difference was large. This large standard deviation may be explained by the heterogeneity of the patient group used in this study. Many PAH patients are likely to have considerable TR, ${ }^{2,37} \mathrm{RBBB}^{7,12,15}$ or both. As shown in Figure 7.6, simulated $\Delta t_{R L, m i n}$ decreased with additional simulation of TR, whereas it increased with RBBB. Coexisting TR and RBBB may also explain the systematic underestimation of RVfw peak shortening $\left(\varepsilon_{\text {RVfw,min }}\right.$ in Figure 7.4 and Table 7.2) in the simulations without RBBB and TR. As shown in Figure 7.6 , simulated $\varepsilon_{\text {RVfw,min }}$ decreased with additional simulation of both TR and RBBB. Unfortunately, the amount of TR was not known for the patient group in this study except for Patient 2. Thus, part of the discrepancy between measured and simulated values of $\varepsilon_{R V f w, m i n}$ (Table 7.2) may be caused by neglecting TR in most of the patient-specific simulations.

In patients with severe PAH, early diastolic lengthening of the septum corresponds to leftward septal bulging during LV isovolumic relaxation. ${ }^{12,19,26,27,35}$ In our regular patient-specific simulations, early diastolic septal lengthening $\left(\Delta \varepsilon_{\text {sept,dias }}\right)$ was systematically underestimated (Figure 7.4 and Table 7.2). Interestingly, the degree of underestimation correlated well with $\Delta \varepsilon_{\text {Sept,dias }}$ as measured in the patients. As shown in Figure 7.5, 7.6, and 7.7, $\Delta \varepsilon_{\text {sept,dias }}$ increased considerably with delay of RVfw mechanical relaxation in the patients with RBBB. Notably, the amount of delay, which was needed to fit $\Delta \varepsilon_{\text {Sept,dias, }}$ increased with measured $\Delta \varepsilon_{\text {Sept,dias. }}$. The latter phenomenon has also been observed in CTEPH patients with and without additional RBBB. ${ }^{15}$ It needs further investigation whether the amount of underestimation of $\Delta \varepsilon_{\text {sept,dias }}$ by our patient-specific simulation procedure is an indicator of myocardial pathology in the RVfw. 
In our simulations of $\mathrm{PAH}$, prolonged mechanical activation of the myofibers in the dilated RV free wall is explained by the normal physiological property of the cardiac muscle that duration of mechanical myofiber activation increases with sarcomere length. This latter property is well known from experiments on isolated cardiac muscle ${ }^{36}$ and is as such implemented in the constitutive equations describing sarcomere mechanics in the TriSeg model. ${ }^{26}$ The systematic underestimation of ventricular mechanical asynchrony in our patient-specific simulations suggests that the abovementioned normal physiological behavior of the cardiac muscle is not sufficient to explain the ventricular mechanical asynchrony as measured in patients with severe PAH. Previous experimental studies showed that severe PAH is associated with structural and electrophysiological remodeling of the RVfw myocardium. . $^{415}$ Ischemia ${ }^{13,38,39}$ and interstitial fibrosis ${ }^{3,15,24}$ in the RV free wall may locally change active and passive myocardial material properties in severe $\mathrm{PAH}$. The disregarding of these pathophysiological aspects in the simulations of severe PAH may partially explain the discrepancy between simulated and measured ventricular mechanical asynchrony.

Besides giving insight in the potential mechanism behind ventricular mechanical asynchrony in PAH patients, the present study also presented a proof of principle showing that the model is feasible for patient-specific simulation of ventricular mechanics in a heterogeneous group of PAH patients. Especially in the subgroup of patients for which additional information about TR and RBBB was used, realistic simulations of local ventricular strain patterns were obtained by fitting the model to a limited number of measured variables. Except for pulmonary artery pressure, all variables were measured non-invasively.

Timing of zero reference strain was defined differently for the measured and the simulated strains. For the simulations, circumferential strain was referred to the status at the moment of aortic valve opening. For the measurements, strain was referred to the circumferential length of the ventricular wall in the first MR tagging image after the ECG R-wave as measured by the MR imaging system. Furthermore, the delay between the true ECG QRS complex and the QRS complex as used by the MR imaging system to trigger image acquisition was unknown. Consequently, measured and simulated strain curves may not be perfectly synchronized. Although this was expected to cause small $(<10 \%)$ systematic differences between measured and simulated values of minimum strain and time to minimum strain in the ventricular walls, it did not affect delay of RVfw minimum strain $\left(\Delta \mathrm{t}_{\mathrm{RL}, \mathrm{min}}\right.$ in Figure 7.1) and early diastolic septal lengthening $\left(\Delta \varepsilon_{\text {sept,dias }}\right.$ in Figure 7.1).

Convergence of the patient-specific fit to steady state was not fast (50-100 
cardiac cycles) and oscillations occurred due to cross-relationships between most adjustable model parameters (PAR in Figure 7.3). Although cross-relations appeared weak (Appendix 7.A), quantitative incorporation of these crossrelations in the fitting procedure can possibly accelerate convergence to steady state. However, computational optimization of the fitting procedure was beyond the scope of the present study.

\section{CONCLUSIONS}

A novel patient-specific fitting procedure has been used to assess ventricular mechanics in a group of patients with $\mathrm{PAH}$. In all patients, patient-specific simulation predicted ventricular mechanical asynchrony as evidenced by delay of RVfw peak shortening and by early diastolic lengthening of the septum. Comparison of measured and simulated ventricular strain patterns revealed a systematic underestimation of early diastolic lengthening of the septum in the simulations. This underestimation suggests that the asynchrony can only partly be explained on the basis of physiological mechanical properties of the myocardial tissue. In a subgroup of PAH patients with RBBB, agreement between measured and simulated ventricular mechanical asynchrony was further improved by additional delay of onset of RVfw mechanical activation. These findings confirm our hypothesis that, in PAH, ventricular mechanical asynchrony is most likely caused by delay of mechanical relaxation of the RVfw. It has been shown that the asynchrony happened irrespective whether the delay of RVfw relaxation is caused by prolonged RVfw mechanical activation, delayed onset of RVfw activation, or both.

\section{ACKNOWLEDGMENTS}

We gratefully acknowledge the financial support of Actelion Pharmaceuticals Nederland B.V. (Woerden, The Netherlands) and the Netherlands Heart Foundation (Grant 2007B203). 


\section{Appendix 7.A: Sensitivity Analysis}

The simulation of the average PAH patient was used as reference simulation for sensitivity analysis. Starting from this reference, one measured variable $V A R_{P A T}$ (Table 7.1) at a time was varied by $10 \%$. For each variation, a simulation was performed with the fitting procedure (Steps 2-4 in the "Methods" section) until steady state was reached. Each of these simulations was used to quantify the relative sensitivities of the model parameters PAR to a change of the measured variable $V A R_{\text {PAT }}$. Relative sensitivity (RS) was defined as:

$$
R S=\frac{\log (\triangle P A R)}{\log \left(\Delta V_{A} R_{P A T}\right)}
$$

A value of 1,0 , and -1 for RS indicates a proportional, independent, and reciprocal relationship, respectively. 
Table 7.A Relative sensitivities of simulated input parameters $V_{A R}$ SIM to changes of model parameters PAR

\begin{tabular}{|c|c|c|c|c|c|c|c|c|}
\hline PAR $\rightarrow$ & $\begin{array}{c}\mathrm{k}_{\mathrm{AO}} \\
{[-]}\end{array}$ & $\begin{array}{c}\mathrm{p}_{\mathrm{AO}} \\
{[\mathrm{kPa}]}\end{array}$ & $\begin{array}{l}k_{P A} \\
{[-]}\end{array}$ & $\begin{array}{c}\text { PDroppulm } \\
{[\mathrm{kPa}]}\end{array}$ & $\begin{array}{l}A_{\text {ref,ll }} \\
{\left[\mathrm{cm}^{2}\right]}\end{array}$ & $\begin{array}{c}A_{\text {ref,RVfw }} \\
{\left[\mathrm{cm}^{2}\right]}\end{array}$ & $\begin{array}{c}\mathrm{CO} \\
{[\mathrm{ml} / \mathrm{s}]}\end{array}$ & $\begin{array}{l}t_{\text {Act }} \\
{[s]}\end{array}$ \\
\hline Reference value & 10.1 & 12.2 & 19.1 & 6.9 & 114 & 154 & 67 & 0.619 \\
\hline \multicolumn{9}{|l|}{ VAR $_{\text {SIM }} \downarrow$} \\
\hline SBP & 2.1 & 0.6 & 0.1 & -0.1 & -0.3 & 0.0 & 0.0 & -0.4 \\
\hline DBP & -1.9 & 0.4 & 0.0 & 0.0 & 0.0 & 0.0 & 0.0 & 0.2 \\
\hline SPAP & 0.0 & 0.0 & 2.8 & 0.2 & 0.0 & -0.1 & 0.0 & 0.0 \\
\hline dPAP & 0.0 & 0.0 & -1.0 & -0.1 & 0.0 & 0.0 & 0.0 & 0.0 \\
\hline mPAP & 0.0 & 0.0 & -1.3 & 1.0 & 0.0 & -0.2 & 0.0 & 0.0 \\
\hline LV EDV & 0.1 & 0.0 & 0.6 & 0.0 & 0.4 & -0.2 & 0.7 & -0.3 \\
\hline RV EDV & 0.0 & 0.0 & 0.0 & 0.0 & 0.0 & 0.5 & 0.1 & 0.0 \\
\hline$\varepsilon_{\mathrm{LVfw}, \min }$ & 0.0 & 0.0 & 0.6 & -0.1 & -0.2 & -0.1 & 0.8 & 0.0 \\
\hline $\mathrm{t}_{\mathrm{LVfw}, \min }$ & -0.4 & 0.1 & 0.0 & 0.0 & 0.1 & 0.1 & 0.0 & 0.9 \\
\hline
\end{tabular}

The absolute reference values of the variable model parameters PAR are derived from the average PAH patient simulation. Grey cells indicate relative sensitivities of the VAR $R_{\text {SIM }}$ - PAR couples as used in the fitting procedure.

Definitions of PAR symbols: $A_{\text {ref, LVVRVfw }}=$ reference area LV wall/RV free wall, LV wall includes LV free wall and septal wall; $\mathrm{CO}=$ cardiac output; $\mathrm{k}_{\mathrm{AO} / \mathrm{PA}}=$ aortic/pulmonary artery stiffness; $\mathrm{p}_{\mathrm{AO}}=$ mean aortic pressure; $p_{\text {Droppulm }}=$ mean pulmonary arteriovenous pressure drop; $t_{A c t}=$ ventricular mechanical activation duration. Definitions of VAR $R_{\text {IIM }}$ symbols: DBP/SBP = diastolic/systolic aortic blood pressure; dPAP/sPAP/mPAP = diastolic/systolic/mean pulmonary artery pressure; EDV = enddiastolic volume; $\mathrm{LV}=$ left ventricular; $\mathrm{RV}$ = right ventricular; $\mathrm{t}_{\mathrm{LVfw}}$ min $=$ time to minimum strain $\mathrm{LV}$ free wall; $\varepsilon_{\mathrm{LVfw}, \min }=$ minimum strain LV free wall. 


\section{Appendix 7.B: Measured vs. Simulated Patient-Specific Ventricular Strain Curves}

This Appendix presents time courses of measured and simulated circumferential strains of the LVfw, Sept, and RVfw for each individual patient.

MEASUREMENT

Patient 1

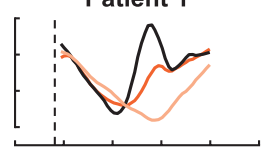

Patient 2

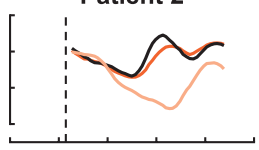

Patient 3
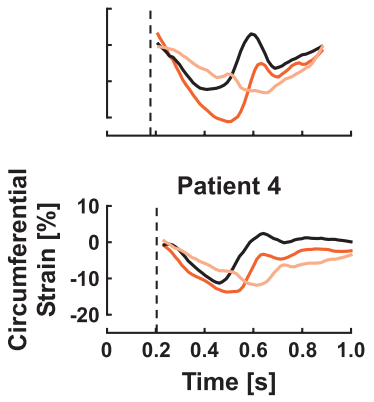

SIMULATION
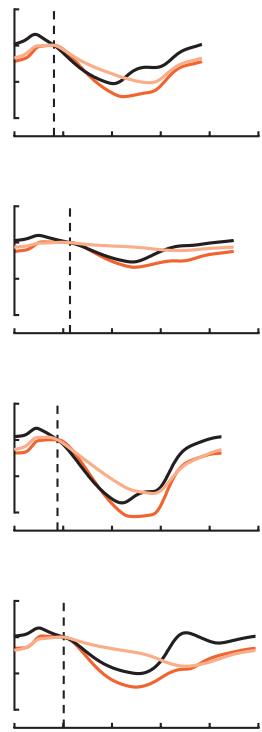

Figure 7.B1: Time courses of measured (left) and simulated (right) circumferential strains in the left ventricular free wall (LVfw), septum (Sept), and right ventricular free wall (RVfw, blue lines) during a cardiac cycle of patients 1-4. Timing of zero strain references are indicated by vertical dashed lines. For the measurements, this reference corresponds to the ECG R-wave. For the simulations, this reference corresponds to the moment of aortic valve opening

LVfw

Sept

RVfw 
MEASUREMENT

Patient 5

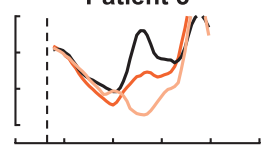

Patient 6

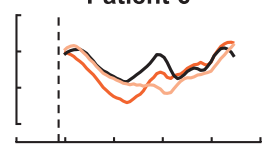

Patient 7

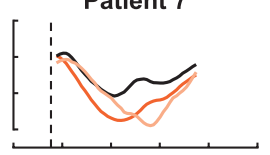

Patient 8

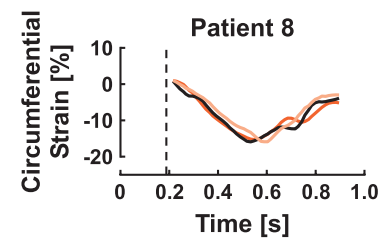

MEASUREMENT

Patient 9

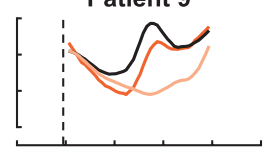

Patient 10

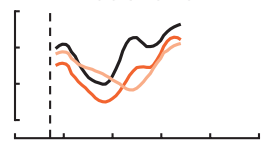

Patient 11
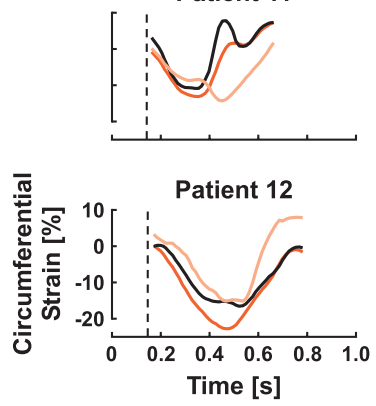

SIMULATION
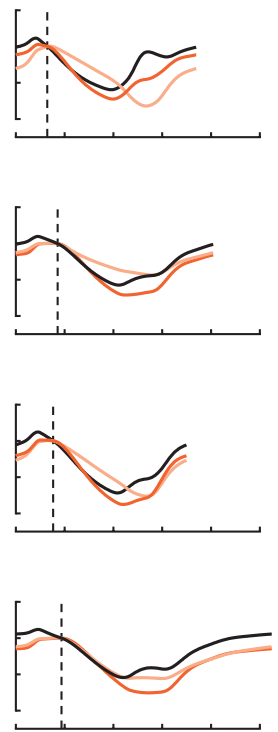

SIMULATION
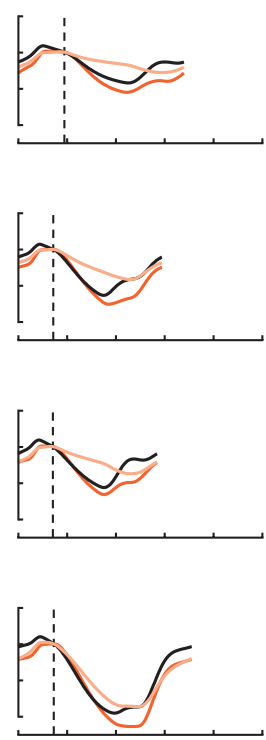

Figure 7.B2: Strain data of patients 5 -

8. Abbreviations and legend details similar as Figure 7.B1.

- LVfw

$\longrightarrow$ Sept

RVfw

Figure 7.B3: Strain data of patients 912. Abbreviations and legend details similar as Figure 7.B1. 


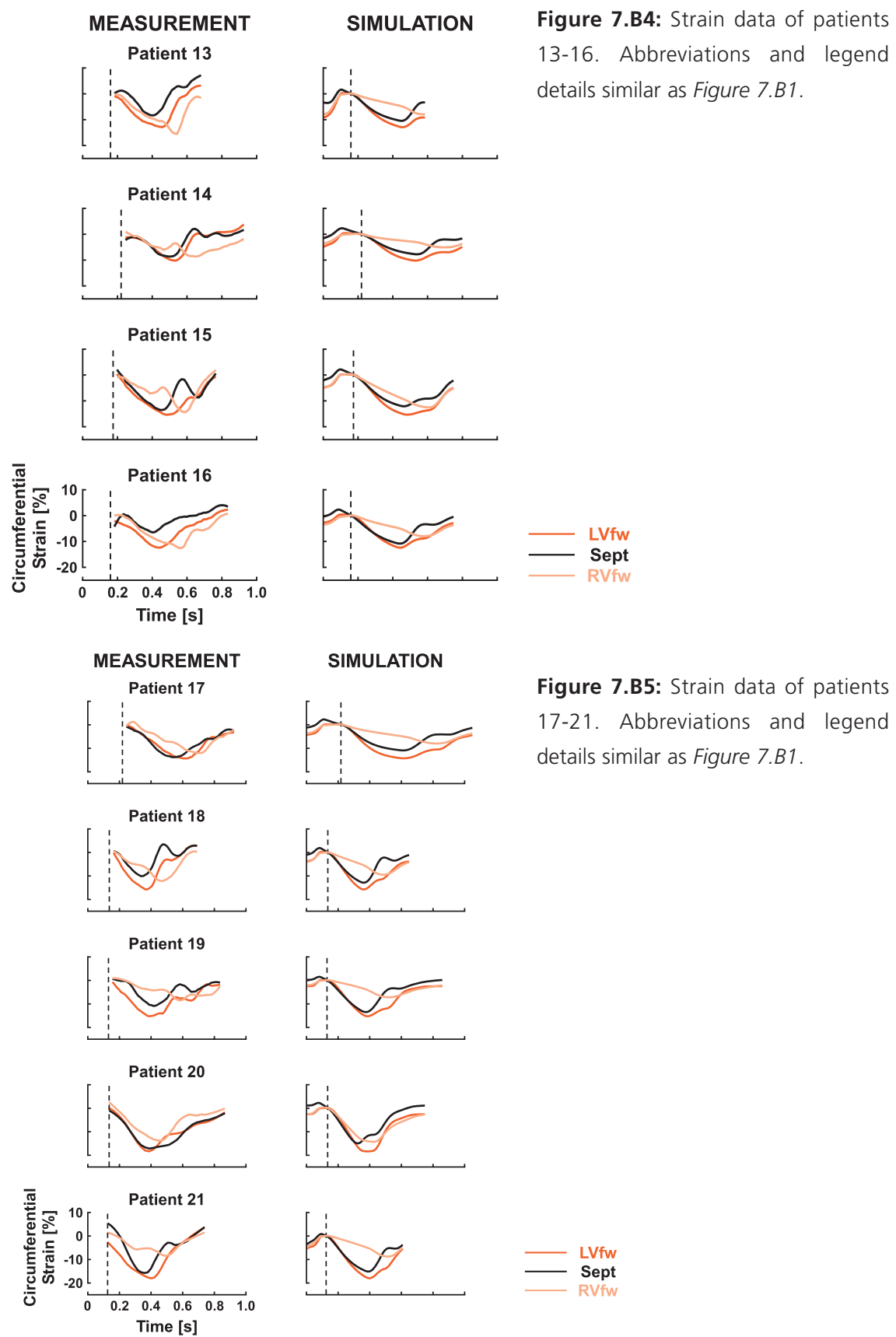




\section{Patient-Specific Modeling in Pulmonary Hypertension}

\section{REFERENCES}

1. Arts T, Delhaas T, Bovendeerd P, Verbeek X, and Prinzen FW. Adaptation to mechanical load determines shape and properties of heart and circulation: The circadapt model. Am J Physiol Heart Circ Physiol 288: H1943-1954, 2005.

2. Berger M, Haimowitz A, Van Tosh A, Berdoff RL, and Goldberg E. Quantitative assessment of pulmonary hypertension in patients with tricuspid regurgitation using continuous wave doppler ultrasound. J Am Coll Cardiol 6: 359-365, 1985.

3. Bishop JE, Rhodes S, Laurent GJ, Low RB, and Stirewalt WS. Increased collagen synthesis and decreased collagen degradation in right ventricular hypertrophy induced by pressure overload. Cardiovasc Res 28: 1581-1585, 1994.

4. Bogaard HJ, Abe K, Vonk Noordegraaf A, and Voelkel NF. The right ventricle under pressure: Cellular and molecular mechanisms of right-heart failure in pulmonary hypertension. Chest 135: 794-804, 2009.

5. Bouchard A, Higgins CB, Byrd BF, 3rd, Amparo EG, Osaki L, and Axelrod R. Magnetic resonance imaging in pulmonary arterial hypertension. Am J Cardiol 56: 938-942, 1985.

6. Chemla D, Antony I, Zamani K, and Nitenberg A. Mean aortic pressure is the geometric mean of systolic and diastolic aortic pressure in resting humans. J Appl Physiol 99: 22782284, 2005.

7. Chen PS, Moser KM, Dembitsky WP, Auger WR, Daily PO, Calisi CM, Jamieson SW, and Feld GK. Epicardial activation and repolarization patterns in patients with right ventricular hypertrophy. Circulation 83: 104-118, 1991.

8. Chin $\mathrm{KM}$, Kim NH, and Rubin LJ. The right ventricle in pulmonary hypertension. Coron Artery Dis 16: 13-18, 2005.

9. de Tombe PP, and ter Keurs HE. Force and velocity of sarcomere shortening in trabeculae from rat heart. Effects of temperature. Circ Res 66: 1239-1254, 1990.

10. Doherty NE, 3rd, Fujita N, Caputo GR, and Higgins CB. Measurement of right ventricular mass in normal and dilated cardiomyopathic ventricles using cine magnetic resonance imaging. Am J Cardiol 69: 1223-1228, 1992.

11. Galie N, Torbicki A, Barst R, Dartevelle P, Haworth S, Higenbottam T, Olschewski H, Peacock A, Pietra G, Rubin LJ, Simonneau G, Priori SG, Garcia MA, Blanc JJ, Budaj A, Cowie M, Dean V, Deckers J, Burgos EF, Lekakis J, Lindahl B, Mazzotta G, McGregor K, Morais J, Oto A, Smiseth OA, Barbera JA, Gibbs S, Hoeper M, Humbert M, Naeije R, and Pepke-Zaba J. Guidelines on diagnosis and treatment of pulmonary arterial hypertension. The task force on diagnosis and treatment of pulmonary arterial hypertension of the european society of cardiology. Eur Heart J 25: 2243-2278, 2004. 


\section{Chapter 7}

12. Gan CT, Lankhaar JW, Marcus JT, Westerhof N, Marques KM, Bronzwaer JG, Boonstra A, Postmus PE, and Vonk-Noordegraaf A. Impaired left ventricular filling due to right-to-left ventricular interaction in patients with pulmonary arterial hypertension. Am J Physiol Heart Circ Physiol 290: H1528-1533, 2006.

13. Gomez A, Bialostozky D, Zajarias A, Santos E, Palomar A, Martinez ML, and Sandoval J. Right ventricular ischemia in patients with primary pulmonary hypertension. J Am Coll Cardiol 38: 1137-1142, 2001.

14. Handoko ML, Lamberts RR, Redout EM, de Man FS, Boer C, Simonides WS, Paulus WJ, Westerhof N, Allaart CP, and Vonk-Noordegraaf A. Right ventricular pacing improves right heart function in experimental pulmonary arterial hypertension: A study in the isolated heart. Am J Physiol Heart Circ Physiol 297: H1752-1759, 2009.

15. Hardziyenka M, Campian ME, Bouma BJ, Linnenbank AC, de Bruin-Bon HA, Kloek JJ, van der Wal AC, Baan J, Jr., de Beaumont EM, Reesink HJ, de Bakker JM, Bresser P, and Tan HL. Right-to-left ventricular diastolic delay in chronic thromboembolic pulmonary hypertension is associated with activation delay and action potential prolongation in right ventricle. Circ Arrhythm Electrophysiol 2: 555-561, 2009.

16. Henkens IR, Mouchaers KT, Vliegen HW, van der Laarse WJ, Swenne CA, Maan AC, Draisma HH, Schalij I, van der Wall EE, Schalij MJ, and Vonk-Noordegraaf A. Early changes in rat hearts with developing pulmonary arterial hypertension can be detected with three-dimensional electrocardiography. Am J Physiol Heart Circ Physiol 293: H13001307, 2007.

17. Henkens IR, Mouchaers KT, Vonk-Noordegraaf A, Boonstra A, Swenne CA, Maan AC, Man SC, Twisk JW, van der Wall EE, Schalij MJ, and Vliegen HW. Improved ecg detection of presence and severity of right ventricular pressure load validated with cardiac magnetic resonance imaging. Am J Physiol Heart Circ Physiol 294: H2150-2157, 2008.

18. Hessel MH, Steendijk P, den Adel B, Schutte Cl, and van der Laarse A. Characterization of right ventricular function after monocrotaline-induced pulmonary hypertension in the intact rat. Am J Physiol Heart Circ Physiol 291: H2424-2430, 2006.

19. King ME, Braun H, Goldblatt A, Liberthson R, and Weyman AE. Interventricular septal configuration as a predictor of right ventricular systolic hypertension in children: A crosssectional echocardiographic study. Circulation 68: 68-75, 1983.

20. Klein SS, Graham TP, Jr., and Lorenz CH. Noninvasive delineation of normal right ventricular contractile motion with magnetic resonance imaging myocardial tagging. Ann Biomed Eng 26: 756-763, 1998.

21. Lee JK, Kodama I, Honjo H, Anno T, Kamiya K, and Toyama J. Stage-dependent changes in membrane currents in rats with monocrotaline-induced right ventricular hypertrophy. Am J Physiol 272: H2833-2842, 1997. 
22. Li GR, Lau CP, Leung TK, and Nattel S. Ionic current abnormalities associated with prolonged action potentials in cardiomyocytes from diseased human right ventricles. Heart Rhythm 1: 460-468, 2004.

23. Lopez-Candales A, Dohi K, Rajagopalan N, Suffoletto M, Murali S, Gorcsan J, and Edelman K. Right ventricular dyssynchrony in patients with pulmonary hypertension is associated with disease severity and functional class. Cardiovasc Ultrasound 3: 23, 2005.

24. Lowes BD, Minobe W, Abraham WT, Rizeq MN, Bohlmeyer TJ, Quaife RA, Roden RL, Dutcher DL, Robertson AD, Voelkel NF, Badesch DB, Groves BM, Gilbert EM, and Bristow MR. Changes in gene expression in the intact human heart. Downregulation of alphamyosin heavy chain in hypertrophied, failing ventricular myocardium. J Clin Invest 100: 2315-2324, 1997.

25. Lumens J, Arts T, Broers B, Boomars KA, van Paassen P, Prinzen FW, and Delhaas T. Right ventricular free wall pacing improves cardiac pump function in severe pulmonary arterial hypertension: A computer simulation analysis. Am J Physiol Heart Circ Physiol 297: H2196-2205, 2009.

26. Lumens J, Delhaas T, Kirn B, and Arts T. Three-wall segment (TriSeg) model describing mechanics and hemodynamics of ventricular interaction. Ann Biomed Eng 37: 22342255, 2009.

27. Marcus JT, Gan CT, Zwanenburg JJ, Boonstra A, Allaart CP, Gotte MJ, and VonkNoordegraaf A. Interventricular mechanical asynchrony in pulmonary arterial hypertension: Left-to-right delay in peak shortening is related to right ventricular overload and left ventricular underfilling. J Am Coll Cardiol 51: 750-757, 2008.

28. McLaughlin VV. Survival in patients with pulmonary arterial hypertension treated with first-line bosentan. Eur J Clin Invest 36 Suppl 3: 10-15, 2006.

29. Naito H, Arisawa J, Harada K, Yamagami H, Kozuka T, and Tamura S. Assessment of right ventricular regional contraction and comparison with the left ventricle in normal humans: A cine magnetic resonance study with presaturation myocardial tagging. $\mathrm{Br}$ Heart J 74: 186-191, 1995.

30. Osman NF, Kerwin WS, McVeigh ER, and Prince JL. Cardiac motion tracking using cine harmonic phase (harp) magnetic resonance imaging. Magn Reson Med 42: 1048-1060, 1999.

31. Reesink HJ, Marcus JT, Tulevski, II, Jamieson S, Kloek JJ, Vonk Noordegraaf A, and Bresser P. Reverse right ventricular remodeling after pulmonary endarterectomy in patients with chronic thromboembolic pulmonary hypertension: Utility of magnetic resonance imaging to demonstrate restoration of the right ventricle. J Thorac Cardiovasc Surg 133: 58-64, 2007.

32. Sandoval J, Bauerle O, Palomar A, Gomez A, Martinez-Guerra ML, Beltran M, and Guerrero ML. Survival in primary pulmonary hypertension. Validation of a prognostic equation. Circulation 89: 1733-1744, 1994. 


\section{Chapter 7}

33. Savitzky A, and Golay MJE. Smoothing and differentiation of data by simplified least squares procedures. Anal Chem 36: 1627-1639, 1964.

34. Simonneau G, Galie N, Rubin LJ, Langleben D, Seeger W, Domenighetti G, Gibbs S, Lebrec D, Speich R, Beghetti M, Rich S, and Fishman A. Clinical classification of pulmonary hypertension. J Am Coll Cardiol 43: 5S-12S, 2004.

35. Tanaka H, Tei C, Nakao S, Tahara M, Sakurai S, Kashima T, and Kanehisa T. Diastolic bulging of the interventricular septum toward the left ventricle. An echocardiographic manifestation of negative interventricular pressure gradient between left and right ventricles during diastole. Circulation 62: 558-563, 1980.

36. ter Keurs HE, Rijnsburger $\mathbf{W H}$, van Heuningen $\mathrm{R}$, and Nagelsmit MJ. Tension development and sarcomere length in rat cardiac trabeculae. Evidence of lengthdependent activation. Circ Res 46: 703-714, 1980.

37. Thistlethwaite PA, and Jamieson SW. Tricuspid valvular disease in the patient with chronic pulmonary thromboembolic disease. Curr Opin Cardiol 18: 111-116, 2003.

38. van Wolferen SA, Marcus JT, Westerhof $N$, Spreeuwenberg MD, Marques KM, Bronzwaer JG, Henkens IR, Gan CT, Boonstra A, Postmus PE, and Vonk-Noordegraaf A. Right coronary artery flow impairment in patients with pulmonary hypertension. Eur Heart J 29: 120-127, 2008.

39. Zong P, Tune JD, and Downey HF. Mechanisms of oxygen demand/supply balance in the right ventricle. Exp Biol Med (Maywood) 230: 507-519, 2005.

40. Zwanenburg JJ, Gotte MJ, Kuijer JP, Heethaar RM, van Rossum AC, and Marcus JT. Timing of cardiac contraction in humans mapped by high-temporal-resolution mri tagging: Early onset and late peak of shortening in lateral wall. Am J Physiol Heart Circ Physiol 286: H1872-1880, 2004.

41. Zwanenburg JJ, Kuijer JP, Marcus JT, and Heethaar RM. Steady-state free precession with myocardial tagging: Cspamm in a single breathhold. Magn Reson Med 49: 722-730, 2003. 
Patient-Specific Modeling in Pulmonary Hypertension 



\section{chapter 0}

General Discussion 
Chapter 8 


\section{INTRODUCTION}

Pulmonary arterial hypertension (PAH) is defined as a group of diseases with a common characteristic histopathological pattern of pulmonary vascular remodeling. This vascular remodeling results in resistive lesions in the pulmonary vascular bed progressively leading to chronic elevation of pulmonary arterial pressure. ${ }^{31}$ Eventually, right ventricular (RV) pressure overload due to PAH leads to impairment of cardiac pump function and premature death.

In this thesis, we aimed to elucidate the mechanisms by which pulmonary arterial hypertension (PAH) affects cardiac pump function. For that purpose, we developed a mathematical model of cardiovascular mechanics incorporating hemodynamic and mechanical ventricular interaction (chapters 3 and 4). It has been shown that the model enables realistic beat-to-beat simulation of global ventricular pump mechanics, dynamics of septal geometry, and myofiber mechanics in the three ventricular walls under normal as well as pulmonary hypertensive loading conditions (chapter 4). In severe PAH, the model predicted similar ventricular mechanical asynchrony as measured in patients. Peak shortening of the right ventricular (RV) free wall was delayed compared to that of the left ventricular (LV) free wall. The interventricular septum lengthened rapidly during the LV isovolumic relaxation phase. We tested pacing of the RV free wall for its potential to treat RV failure in patients with severe PAH (chapter 5). These model simulations suggested that early pacing of the RV free wall reduces ventricular mechanical asynchrony and, thereby, improves RV pump function. Furthermore, the model was used to simulate ventricular mechanics in patients with chronic thromboembolic pulmonary hypertension before and after pulmonary thromboendarterectomy (chapter 6). Pathology-specific simulations predicted similar improvement of LV filling hemodynamics (increase of mitral E/A-ratio) after pulmonary thromboendarterectomy as measured in a group of patients. It has also been shown that the increase of mitral E/A-ratio with pulmonary thromboendarterectomy is predominantly caused by increase of RV systolic output (hemodynamic ventricular interaction) and to a lesser degree by abolishment of leftward septal bulging (mechanical ventricular interaction). Finally, a patient-specific simulation procedure was designed for assessment of ventricular mechanics and hemodynamics in the individual PAH patient (chapter 7). With patient-specific simulation in a group of PAH patients with and without right bundle branch block, it is shown that ventricular mechanical asynchrony is most likely caused by delay of mechanical relaxation of the RV free wall either due to prolonged mechanical activation of the RV free wall, delayed onset of mechanical activation, or both. 


\section{Chapter 8}

In this concluding chapter, we will put the findings as presented in the previous chapters in a broader perspective. We will also focus on study limitations, potential clinical implications, and future studies as derived from our study results.

\section{Modeling Mechanical Ventricular Interaction Using the TriSeg Model}

In the human heart, direct anatomical coupling of the ventricular walls results in mechanical ventricular interaction. Consequently, a change in loading condition of either ventricle directly influences pump function of the other ventricle mediated, among others, by the interventricular septum. ${ }^{3}, 26,76,85,98$ Experimental as well as clinical studies showed that septal geometry and motion are directly related to septal transmural pressure difference. ${ }^{21,26,46,56,79}$ Septal geometry and motion appeared to be of diagnostic and predictive significance in patients with asynchronous activation of the ventricular walls ${ }^{34,56,77}$ and with PAH. ${ }^{21,41,45,80}$

We developed the mathematical TriSeg model for the assessment of LV and RV mechanics and hemodynamics under normal and pathological ventricular loading conditions (chapter 3 and 4). Three ventricular wall segments representing the LV free wall, the septum, and the RV free wall are mechanically coupled on the basis of tensile force equilibrium in their junction. Global LV and RV pump mechanics are related to representative myofiber mechanics in the three ventricular walls, satisfying the principle of conservation of energy. The TriSeg model is embodied as a ventricular module in the lumped CircAdapt model describing mechanics and hemodynamics of the whole human circulation. ${ }^{2}$ The latter model provides the required hemodynamic environment for the ventricles as represented by the TriSeg model, i.e., atrial walls, cardiac valves, large blood vessels, and peripheral resistances. The combined CircAdapt/TriSeg model allows beat-to-beat simulation of time-dependent ventricular mechanics and hemodynamics such as volumes and pressures of the cardiac cavities, geometries and myofiber mechanics of the cardiac walls, and flows through the valves. Furthermore, the model allows adaptation of size and mass of cardiac walls and large blood vessels to normalize local mechanical load to tissue-specific physiological levels.2, 64

In a first trial (chapter 3), the model was used to simulate ventricular mechanics in the canine heart with synchronous mechanical activation of the three ventricular walls and with asynchronous mechanical activation due to left bundle branch block (LBBB). The simulated results of ventricular mechanics and hemodynamics under normal and LBBB conditions were compared with experimental data obtained before and after acute induction of LBBB in dogs, 
respectively. Similarly as observed in dogs with $\mathrm{LBBB}, 57,78,95$ the model predicted 1) delay of pressure rise and decay in the LV as compared to those in the RV, 2) increase of asymmetry of the RV pressure curve, 3) early systolic leftward motion of the septum, and 4) increase of myofiber stroke work in the late activated LV free wall. The surprisingly good agreement between simulations and measurements suggested that the TriSeg model allows realistic simulation of ventricular mechanics including mechanical ventricular interaction and, thereby, extends the application range of the CircAdapt model.

We also simulated ventricular mechanics and hemodynamics in the human heart with normal and increased pulmonary vascular resistance (chapter 4). With normal pulmonary vascular resistance, the model predicted realistic ventricular geometry as obtained by adaptation of ventricular wall masses and sizes so that mechanical myofiber stress and strain were normalized to known physiological levels. LV and RV end-diastolic cavity volume were about equal, ${ }^{84}$ whereas the LV free wall and the septum were about 3 times thicker than the RV free wall. ${ }^{24,36,}$ ${ }^{87}$ Also LV and RV pressure-volume loops and time courses of blood flow velocities through the cardiac valves were in agreement with measurements obtained in healthy humans and experimental animals. ${ }^{30,} 39,47,83,91,92$ With increase of pulmonary vascular resistance including load adaptation of the ventricular walls, model simulations showed similar changes of ventricular wall thicknesses, septal geometry, and blood flow patterns through RV valves as observed in patients and experimental animals with PAH. RV free wall thickness increased, whereas thickness of the LV free wall and septal wall hardly changed. ${ }^{75}$ Septal curvature decreased linearly with pressure difference across the septum. ${ }^{14,21,25,26,45,46,80}$ Acceleration time of pulmonary flow velocity (time to peak flow velocity) decreased linearly with increase of mean pulmonary artery pressure. ${ }^{13,20}$ Furthermore, RV isovolumic contraction and relaxation times increased while pulmonary ejection time decreased. ${ }^{91}$, 99 When increasing pulmonary vascular resistance without load adaptation of the myocardial tissue, the model predicted similar changes of ventricular mechanics and geometry as often associated with RV failure in patients with severe $\mathrm{PAH}^{17,40,69}$ i.e., RV dilatation and rapid leftward septal motion following LV ejection.

Based on all these similarities between measurements and simulations (chapter 3 and 4), it is concluded that the model is a valuable simulation tool for assessment of time-dependent ventricular mechanics and hemodynamics in patients with PAH. Moreover, the model is applicable in a wide range of disease severity due to its possibility to apply adaptation of the myocardial tissue to mechanical load. 


\section{Right Ventricular Free Wall Pacing in Pulmonary Arterial Hypertension: Treatment of Right Ventricular Failure?}

In PAH, peak shortening of the RV free wall is delayed as compared to that of the LV free wall and septum. This ventricular mechanical asynchrony appears related to leftward septal bulging, stroke volume, RV free wall stress, disease severity, and functional class of the patient. ${ }^{58,69}$ As a potential treatment, RV resynchronization therapy has been suggested to improve ventricular mechanical synchrony and, thereby, RV pump function in PAH. $7,42,58,69$

In chapter 5, the TriSeg model is used to predict the effect of early RV free wall pacing on ventricular pump mechanics and hemodynamics in PAH. Severe PAH was simulated by increasing RV pressure load without adaptation (decompensation). This simulation showed many similarities with data obtained in patients with severe PAH, i.e., RV dilatation, delayed peak shortening of the RV free wall as compared to that of the LV free wall and septum, and leftward septal bulging. Furthermore, the model predicted an inhomogeneous distribution of mechanical myofiber load over the ventricular walls. Myofiber work in the RV free wall was three times higher than that in the septum and the LV free wall. Interestingly, preexcitation of the RV free wall by pacing decreased the exorbitantly high myofiber work in the RV free wall and increased myofiber work in the LV free wall and septum. Apparently, the mechanical interaction of the ventricular walls allowed redistribution of myofiber work over the ventricular walls. An optimal time of RV free wall preexcitation was found resulting in most homogeneous distribution of mechanical load over the ventricular walls, best synchrony of RV and LV pressure curves, and moderate improvement of RV pump function, as evidenced by increase of $\mathrm{RV} \mathrm{dP} / \mathrm{dt}_{\max }$, decrease of RV end-diastolic pressure, and decrease of RV enddiastolic volume. These simulations suggested that early pacing of the RV free wall is a potential treatment of RV failure in patients with severe PAH.

The beneficial effect of early RV free wall pacing on RV pump function in PAH is supported by experimental data obtained by Handoko et al. ${ }^{37}$ in isolated hearts of rats with PAH and RV failure. Similarly as observed in our simulations, an optimal pacing setting with RV free wall preexcitation was found resulting in maximal improvement of RV systolic function ( $8.5 \%$ increase of RV dP/dt $\left.t_{\max }\right)$ and resynchronization of $L V$ and RV pressure curves. Furthermore, in the study by Handoko et al., ${ }^{37}$ RV free wall pacing did not detrimentally affect LV pump function or coronary perfusion. Thus, the results of our simulation study in chapter 5 and of the experimental study by Handoko et al. ${ }^{37}$ mutually support and complement each other. 
In our model, each ventricular wall is lumped into a spherical wall segment containing a single contractile fiber describing representative passive and active sarcomere properties of the entire wall. ${ }^{64}$ Despite this simplified representation of the ventricular walls, the qualitative and quantitative agreement between our simulations and experimental as well as clinical observations indicate that the simplifications made in the simulations may not obscure first-order effects of RV free wall pacing on ventricular mechanics and pump function in severe PAH. The study in chapter 5 only focuses on the acute hemodynamic effects of pacing. Long-term effects are not considered. However, in analogy with cardiac resynchronization in dyssynchronous ventricles, acute hemodynamic effects can be expected to be maintained over time and may even lead to further improvements by reverse remodeling. ${ }^{11}$

\section{Abnormal Left Ventricular Filling Hemodynamics in Pulmonary Arterial Hypertension: a Result of Underfilling or Compression by Septal Bulging?}

In patients with PAH, chronic RV pressure overload is associated with reduced LV end-diastolic volume and abnormal LV filling pattern, 22, 32, 35, 59, 67, 70, 72, 96 i.e., peak blood flow velocity of the early passive mitral filling wave (E-velocity) is smaller than that of the atrial contraction-induced late active filling wave (Avelocity). This phenomenon might be due to leftward septal bulging, which is believed to hinder passive filling by compressing the LV (mechanical ventricular interaction). Alternatively, the inability of the RV to maintain normal stroke volume is believed to reduce preload of the LV (hemodynamic ventricular interaction). It is still unclear to what extent both mechanisms contribute to impairment of LV filling in PAH patients. In chapter 6 , the separate contributions of both mechanisms are quantified in patients with chronic thromboembolic pulmonary hypertension (CTEPH) to obtain better understanding of the mechanism by which PAH affects LV filling.

In a group of CTEPH patients, transmitral blood flow velocities and RV hemodynamic data were obtained before and after pulmonary thromboendarterectomy (PTE). The TriSeg model was fitted to preoperative average values of cardiac output (CO), mean pulmonary artery pressure (mPAP), mitral E/A-ratio, and mean left atrial pressure (mLAP). Starting from this preoperative reference state with leftward septal bulging, PTE was simulated by changing mPAP and CO to average postoperative values. The effect of PTE on LV filling hemodynamics as predicted by our model was surprisingly similar to the effect 


\section{Chapter 8}

as observed in patients with CTEPH. Mitral E/A-ratio, mitral E-velocity, LV enddiastolic volume, and septal curvature increased from subnormal values in CTEPH before PTE to normal values after PTE, whereas RV end-diastolic volume decreased to normal value. With our pathology-specific modeling approach, effects of RV pressure overload and of RV systolic output on LV filling hemodynamics could be discriminated quantitatively by simulating either an exclusive decrease of mPAP or an exclusive increase of $\mathrm{CO}$, respectively. With exclusive decrease of mPAP, mitral E/A-ratio increased 26\%, mLAP decreased $16 \%$, and leftward septal bulging was abolished. With exclusive increase of $\mathrm{CO}$, mitral E/A-ratio increased 53\%, mLAP increased 62\%, whereas leftward septal bulging persisted. These data show that increase of mitral E/A-ratio with PTE in CTEPH patients is largely the effect of increased RV systolic output. Abolishment of leftward septal bulging appears to be of less importance.

In patients with chronic RV pressure overload, cardiac output may be decreased due to many functional and morphological alterations of both the RV and the LV. As has been shown for PTE, 22, 23, 35, 67, 71, 72 lung transplantation, ${ }^{16}$ and repair of congenital heart disease, ${ }^{65,66} \mathrm{LV}$ hemodynamic and morphological changes as a result of $\mathrm{RV}$ pressure overload remain largely reversible, even after years of illness. The acute reversibility of LV filling hemodynamics after PTE strongly suggests a functional rather than a structural change of the LV myocardium with RV pressure overload due to CTEPH. This concept is confirmed by the simulations of decompensated PAH in chapters 4 and 5 showing that RV pressure overload mostly increases mechanical myofiber load in the RV free wall, whereas it hardly affects the myofiber stress-strain relation in the LV free wall and the interventricular septum.

In animal models with chronic RV pressure overload, LV systolic function (contractility) appeared unaffected. ${ }^{27,50,51,54}$ Thus, increase of CO with PTE is most likely the result of improved RV systolic performance. This latter improvement may have several causes. First, decrease of RV afterload in decompensated pulmonary hypertension results in reduction of interventricular mechanical dyssynchrony ${ }^{61,66,69}$ and, thus, in a more coordinated contraction of the three ventricular walls with less leftward septal bulging. This may, therefore, account for an indirect effect of decreased leftward septal bulging on LV filling hemodynamics via improvement of RV systolic output. Second, in patients with CTEPH, decrease of RV afterload by PTE has been shown to significantly reduce tricuspid regurgitation. ${ }^{81}$ Third, PTE and the associated decrease of RV enddiastolic volume may result in an acute reduction of total heart volume and, thus, of pericardial constraint. Acute reduction of pericardial constraint has been 
shown to increase $\mathrm{CO}$ in various animal models of pulmonary hypertension. ${ }^{5,6}$ Finally (and speculatively), PTE may lead to improvement of RV coronary perfusion. Exorbitantly high RV pressure may increase intramyocardial pressure in the RV free wall and, thus, increase resistance of the RV coronary vasculature more than that of the LV. This may result in relatively reduced myocardial perfusion of the RV. The acute decrease of RV pressure with PTE may result in a decrease of RV coronary vascular resistance (facilitating RV myocardial perfusion) and also cause a decrease in RV mechanical myofiber load. Consequently, RV contractile function and stroke volume can improve.

Many studies have reported strong correlations between septal curvature and LV filling parameters such as E/A-ratio and speculated on the potential detrimental effect of leftward septal bulging on LV filling and RV performance in patients with pulmonary hypertension. ${ }^{22,52,59,67,72,74,86}$ The results of our study strongly support the concept that perturbations in LV filling pressure, output, and Doppler flow patterns due to CTEPH are primarily caused by diminished RV outflow and, hence, LV underfilling. Although not negligible, the relative contribution of septal geometry and bulging is considerably less. Also, the increases in cardiac output and LV filling explain the marked changes in LV Doppler diastolic filling pattern after PTE, rather than changes in septal geometry per se.

\section{Patient-Specific Modeling to Assess Ventricular Mechanical Asynchrony in Pulmonary Arterial Hypertension}

In $\mathrm{PAH}$, the most distinct characteristic is the ventricular mechanical asynchrony as expressed by delay of peak shortening of the RV free wall and by early diastolic lengthening of the interventricular septum. In previous clinical studies, this ventricular mechanical asynchrony appeared related to disease severity and functional class of the patient. ${ }^{58,69}$ Moreover, model simulations (chapter 5$)^{61}$ as well as experimental data obtained in isolated hearts of rats with PAH37 show that early pacing of the RV free wall may decrease ventricular mechanical asynchrony and, thereby, improve RV pump function. Thus, ventricular mechanical asynchrony appears of significant diagnostic and therapeutic importance in patients with PAH. However, the precise mechanism by which PAH leads to ventricular mechanical asynchrony is poorly understood.

In chapter 7, a novel patient-specific fitting procedure is presented and applied to assess ventricular mechanics in a heterogeneous patient group consisting of PAH and CTEPH patients with and without right bundle branch 
block (RBBB). Patient-specific simulations showed ventricular mechanical asynchrony in all patients. This asynchrony was evidenced by delay of peak shortening of the RV free wall and by early diastolic lengthening of the septum. Comparison of measured and simulated ventricular strain patterns revealed a systematic underestimation of early diastolic lengthening of the septum in the simulations. This underestimation suggests that the mechanical asynchrony can only partly be explained on the basis of physiological mechanical properties of the myocardial tissue. In a subgroup of patients with RBBB, agreement between measured and simulated ventricular mechanical asynchrony was further improved by additional delay of mechanical activation of the RV free wall. These findings suggest that, in $\mathrm{PAH}$, ventricular mechanical asynchrony is caused by delay of mechanical relaxation of the RVfw. It has been shown that the asynchrony happened irrespective whether the delay of RVfw relaxation is caused by prolonged RVfw mechanical activation, delayed onset of RVfw activation, or both.

The suggested causality between delay of RVfw relaxation and ventricular mechanical asynchrony in PAH is supported by the findings of several clinical and experimental studies showing that chronic RV pressure overload is associated with significant electrophysiological remodeling in the myocardium of the RV free wall. ${ }^{18,38,53,55}$ In patients with CTEPH, it has been shown that action potential duration is increased and conduction velocity is decreased in the RV free wall compared to those in the LV free wall. ${ }^{18,} 38$ Moreover, ventricular mechanical asynchrony appeared to be related to the time difference between $L V$ and RV onset of electrical activation, the difference between LV and RV action potential duration, and the time difference between LV and RV repolarization. Several sarcolemmal ion currents are known to be significantly changed in the RV free wall of patients and experimental animals with chronic RV pressure overload. ${ }^{53,55}$ The impact of electrophysiological remodeling as a result of RV pressure overload on global cardiac pump function in patients with PAH is hard to assess in vivo. In the future, more insight in the mechanism by which PAH leads to delay of mechanical myofiber relaxation in the RV free wall and, hence, ventricular mechanical asynchrony may be obtained by model simulations. For that purpose, the present model of sarcomere mechanics in the TriSeg model (chapter 4, Appendix 4.B) should be replaced by a more complex model describing subcellular electrophysiology and mechanoelectric feedback. ${ }^{48,} 49$

In our patient-specific simulations based on normal physiological mechanical properties of the myocardial tissue (chapter 7), early diastolic septal lengthening was systematically underestimated. Interestingly, the degree of 
underestimation correlated well with early diastolic septal lengthening as measured in the patients. It needs further investigation whether the amount of underestimation of early diastolic septal lengthening by our patient-specific simulation procedure is an indicator of myocardial pathology in the RV free wall. For that purpose, monitoring septal geometry and motion may be of considerable importance, since it has been shown that early diastolic lengthening of the septum corresponds to leftward septal bulging during LV isovolumic relaxation in our simulations of PAH (chapters 4 and 5) as well as in patients with severe $\mathrm{PAH}^{32,45,64,69,90}$

\section{StUdy LiMitations}

In the TriSeg model, ventricular geometry is approximated by three thick-walled spherical segments encapsulating the LV and RV cavities (Figure 4.2A). In reality, LV geometry may be considered to be a thick-walled truncated ellipsoid. The relatively thin-walled RV free wall partially encapsulates the $L V$, giving the RV cavity a crescent shape when viewed in short-axis view (Figure 2.5). At the base, the ventricular cavities are enclosed by the non-contractile basal sheet with valves. ${ }^{88}$ The simplification to spherical segments without a non-contractile sheet may have resulted in systematic errors of ventricular dimensions. Although there are indications of the importance of myocardial bending stiffness for simulation of septal geometry, ${ }^{8}{ }^{26}$ we neglected this effect for simplicity. Despite these inaccuracies, simulated ventricular hemodynamics as well as relative changes of ventricular dimensions during the cardiac cycle were surprisingly realistic. The fact that ventricular mechanics and hemodynamics are simulated so realistic is most likely a result of adaptation and of strict application of laws stating conservation of energy as applied to ventricular wall and cavity mechanics.

A crucial step in the Triseg model is the calculation of myofiber strain from ventricular wall geometry in terms of midwall surface area and curvature (Figure 4.3). The one-fiber model by Arts et al., ${ }^{1}$ related myofiber strain to the ratio of cavity volume to wall volume. This model has previously been shown to be applicable to an anisotropic thick-walled structure like a myocardial wall when assuming rotational symmetry and homogeneity of mechanical load in the wall. In the TriSeg model, the relation between midwall area and myofiber strain was derived by applying the one-fiber model to a closed spherical cavity (chapter 4, Eq. 4.15 and Appendix 4.A). The resulting relation was then extended to a partial segment of the sphere by considering a fraction of the wall, having the same curvature, wall tension, and transmural pressure difference. The original 
one-fiber model has been shown to be rather insensitive to wall geometry. ${ }^{1}$ We expected the present relation between midwall area, curvature, and transmural pressure also to be quite insensitive to actual geometry. However, this fact has not been proven.

Animal studies have shown that the functional effect of asynchronous electrical activation is dramatic because the various regions differ not only in the time of onset of mechanical activation, but also in the pattern of contraction. ${ }^{78}$ Early activated regions stretch not-yet activated remote regions. This stretching further delays shortening of the late-activated regions and increases their force of local contraction by virtue of the (local) Frank-Starling mechanism. Because of their vigorous contraction, the late-activated regions impose loading on the earlier activated territories, which may undergo systolic paradoxical stretch. This inhomogeneous pattern of contraction and stretching has been shown to cause a hemodynamically less effective and energetically less efficient ventricular contraction. ${ }^{4}$ This phenomenon is also observed in our simulations of cardiac mechanics with left bundle branch block (chapter 3 and Figure 3.6) showing that mechanical myofiber work is significantly increased in the late-activated LV free wall and decreased in the earlier activated septum. These simulations indicate that mechanical ventricular interaction of the three ventricular walls is a very important mechanism for the hemodynamic changes associated with asynchronous mechanical activation of these walls. Due to its lumped character, the TriSeg model did not allow description of regional interactions within each wall segment but was limited to the description of its average representative myofiber mechanics. Especially with pacing of the dilated RV free wall of patients with severe PAH (chapter 5), the potential effect of heterogeneity of contractility and onset of activation within a single ventricular wall due to activation spread is not covered by the Triseg model.

Although the TriSeg model can be easily modified to include a description of inhomogeneous myocardial wall properties, a finite element model of cardiac mechanics is more accurate and better suited to describe local inhomogeneities in mechanical load. For patient-specific modeling of the circulation, the model should simulate many cycles in order to find a best match with the available set of measurements. For that purpose, the Triseg model is to be preferred because it allows almost real-time simulation of ventricular mechanics and hemodynamics, while calculation effort of a finite element model of both ventricles is more than 1,000 times larger. ${ }^{44}$ The finite element model is absolutely needed to estimate and evaluate possible errors introduced by the applied simplifications of the TriSeg model. 
In the present thesis, the external pressure surrounding the LV and RV free walls is assumed to be zero. The real heart is surrounded by the pericardium, which constrains increase of total heart volume during volume overload. Under normal resting conditions, the pericardium is believed to play a minor role, setting pericardial pressure close to zero. ${ }^{68,73,97}$ Also under chronic PAH conditions, the effect of the pericardium on ventricular mechanics is likely to be of minor importance, because measurements demonstrated adaptive dilatation of the pericardium in patients and experimental animals with chronic RV dilatation. ${ }^{10,28}$ However, with acute increase of total heart volume, the pericardium affects cardiac hemodynamics and interaction of the cardiac chambers significantly. 5, 15, 29 In our simulations of acute RV pressure overload in chapter 4 , the effect of the pericardium should be considered because of severe RV dilatation (Figure 4.5) and increase of total heart volume. In our simulations of acute interventions in patients with PAH such as pacing (chapter 5) or pulmonary thromboendarterectomy (chapter 6), however, the effect of the pericardium is assumed to be negligible because these interventions lead to decrease of RV cavity volume.

An important limitation of our model is the fact that heart rate, ventricular stroke volume, and, hence, cardiac output are prescribed independent model parameters. In reality, however, these parameters are mutually dependent and are tightly controlled for maintenance of mean arterial blood pressure. As a result of structural adaptation, the ventricles of the normal heart are able to deliver the mechanical pump work needed to maintain normal arterial blood pressure under a wide range of loading conditions (exercise). In patients with severe PAH, exorbitant RV pressure overload increases mechanical myofiber load in the RV free wall above the level that can be compensated for by adaptation. Several studies showed that morbidity and mortality of PAH patients are determined by the ability of the RV to maintain normal stroke volume and cardiac output. ${ }^{19,82}$ Unfortunately, the TriSeg model cannot be used to predict changes of cardiac output as a result of therapeutic interventions such as RV free wall pacing in severe PAH (chapter 5) or pulmonary thromboendarterectomy in CTEPH (chapter 6).

In our simulations of severely decompensated PAH (chapter 5), mechanical myofiber load was inhomogeneously distributed over the ventricular walls. In the RV free wall, myofiber stroke work was increased to almost three times normal values, whereas myofiber stroke work was decreased by about $20 \%$ in the LV free wall and the septum. When assuming stroke work density to be correlated to oxygen consumption, ${ }^{89}$ the latter redistribution of myofiber stroke work implies a considerable increase of oxygen demand of the myocardium in 
the RV free wall. Since oxygen supply is not included in our model, the potential effect of coronary perfusion imposed limitations on myocardial performance is unknown in our simulations of pulmonary hypertension.

With severe PAH, simulated ventricular mechanical asynchrony was systematically underestimated as compared to the asynchrony measured in patients (chapters 5 and 7). In our simulations of PAH, prolonged mechanical activation of the myofibers in the dilated RV free wall is explained by the normal physiological property of the cardiac muscle that duration of mechanical myofiber activation increases with sarcomere length. This latter property is well known from experiments on isolated cardiac muscle ${ }^{93}$ and is as such implemented in the constitutive equations describing sarcomere mechanics in the TriSeg model (chapter 4, Appendix 4.B). ${ }^{64}$ The systematic underestimation of ventricular mechanical asynchrony in the patient-specific simulations in chapter 7 suggests that the abovementioned normal physiological behavior of the cardiac muscle is not sufficient to explain the ventricular mechanical asynchrony as measured in patients with severe PAH. Previous experimental studies showed that severe PAH is associated with structural and electrophysiological remodeling of the RVfw myocardium. ${ }^{12,38}$ Ischemia $^{33,94,100}$ and interstitial fibrosis ${ }^{9,38,60}$ in the RV free wall may locally change active and passive myocardial material properties in severe $\mathrm{PAH}$. The disregarding of these pathophysiological aspects in the simulations of severe PAH may partially explain the discrepancy between simulated and measured ventricular mechanical asynchrony.

\section{Future Perspectives}

The simulation results presented in chapter 5 may provide a theoretical framework for a novel therapeutic strategy for severely decompensated PAH patients. Although decrease of pulmonary vascular resistance remains the primary therapeutic target in PAH patients, pacing of the RV free wall as an additional treatment may contribute to clinical improvement of the patient by unloading the myocardial tissue in the RV free wall and, thereby, delaying development of RV failure. Furthermore, it might be useful as bridge to thoracic organ transplantation in these patients. More experimental and clinical evidence is needed to prove that the predicted beneficial effect of RV free wall pacing is clinically relevant for a PAH patient.

Simulation results suggest that, in severe PAH, ventricular mechanical asynchrony is a direct consequence of increased myofiber stress in the RV free wall because of incomplete adaptation to increased load (chapter 5 ). Therefore, 
it seems safe to assume that the ventricular mechanical asynchrony as measured in patients with severe PAH also indicates RV decompensation (chapter 7). ${ }^{69} \mathrm{~A}$ spin-off of the present thesis may therefore be that ventricular mechanical asynchrony can be used as an index of RV decompensation.

Besides giving insight in the potential mechanism behind ventricular mechanical asynchrony in PAH patients, the study in chapter 7 also presented a proof of principle showing that the model is feasible for patient-specific simulation of ventricular mechanics in a heterogeneous group of PAH patients. Especially in the subgroup of patients for which additional information about tricuspid regurgitation and right bundle branch block was used, realistic simulations of local ventricular strain patterns were obtained by fitting the model to a limited number of measured variables. Except for pulmonary artery pressure, all variables were measured non-invasively. Currently, the model is evaluated for its potential to patient-specifically simulate ventricular hemodynamics and mechanics in PAH patients using exclusively non-invasive measurements as input. For that purpose, non-invasive quantification of RV pressure is needed. In chapter 4 , it has been shown that our model describes a linear relation between septal curvature and the pressure difference across the septum. This relation appeared similar to the one measured in a group of healthy adults and patients with PAH. ${ }^{21,64,79}$ Since it is relatively easy to image septal geometry non-invasively, we expect that it may be possible by patientspecific simulation to estimate RV pressure on the basis of exclusively noninvasive measurements. Such approach may pave the way for completely noninvasive patient-specific simulation of ventricular hemodynamics and mechanics in patients with PAH. Although we realize that invasive right heart catheterization will be necessary for clinical diagnosis of $\mathrm{PAH}$, we think that a modeling approach may be useful for non-invasive patient-specific follow-up by monitoring the effect of therapeutic interventions. It may also enable a priori patient-specific quantification of the effect of therapeutic interventions such as pacing or thromboendarterectomy on cardiac pump function.

As shown in this thesis, the CircAdapt/TriSeg environment is versatile and flexible by its modular setup and enables realistic simulation of cardiovascular mechanics and hemodynamics under normal as well as various pathological conditions. Although the present thesis primarily focuses on PAH, previous trials $^{43,62,63}$ showed that the model may also be a useful tool for in silico testing of hypotheses related to clinical and physiological issues in the field of cardiac resynchronization therapy. 


\section{General Conclusions}

In this thesis, we gained more insight in the mechanisms by which pulmonary arterial hypertension (PAH) affects left ventricular (LV) and right ventricular (RV) pump function. We presented a novel mathematical model of cardiovascular mechanics incorporating hemodynamic and mechanical ventricular interaction. This model simulates ventricular mechanics and hemodynamics realistically under normal as well as pulmonary hypertensive ventricular loading conditions. With severe $\mathrm{PAH}$, the model predicts similar ventricular mechanical asynchrony as measured in patients. Model simulations suggest that early pacing of the RV free wall in patients with severe PAH improves interventricular mechanical synchrony, decreases mechanical myofiber load in the RV free wall, and improves RV pump function. Furthermore, the model is used to assess the mechanism by which acute decrease of RV pressure overload by pulmonary thromboendarterectomy improves filling hemodynamics of the LV in patients with chronic thromboembolic pulmonary hypertension. These simulations show that increase of mitral E/A-ratio with pulmonary thromboendarterectomy is predominantly caused by increase of RV systolic output (hemodynamic ventricular interaction). The contribution of abolishment of leftward septal bulging due to decrease of RV pressure overload (mechanical ventricular interaction) is considerably less. Finally, a patient-specific simulation procedure is designed for assessment of ventricular mechanics and hemodynamics in the individual PAH patient. Patient-specific simulations are obtained by fitting the model to a limited set of measurements obtained in a heterogeneous group of PAH patients. Agreement between simulated and measured ventricular strain patterns shows that ventricular mechanical asynchrony can only partly be explained by normal physiological mechanical properties of the myocardial tissue. In a subgroup of PAH patients with right bundle branch block, agreement between simulated and measured ventricular mechanical asynchrony was further improved by introduction of an additional delay of mechanical activation of the RV free wall. These patient-specific simulations show that ventricular mechanical asynchrony is most likely caused by delay of mechanical relaxation of the RV free wall either due to prolonged mechanical activation of the RV free wall, delayed onset of mechanical activation, or both. The model presented in this thesis has been shown to be a valuable simulation tool for elucidating mechanisms of cardiac pathophysiology in PAH and for patient-specific assessment of ventricular mechanics and hemodynamics in patients with PAH. 


\section{REFERENCES}

1. Arts T, Bovendeerd PH, Prinzen FW, and Reneman RS. Relation between left ventricular cavity pressure and volume and systolic fiber stress and strain in the wall. Biophys J 59: 93-102, 1991.

2. Arts T, Delhaas T, Bovendeerd P, Verbeek X, and Prinzen FW. Adaptation to mechanical load determines shape and properties of heart and circulation: The circadapt model. Am J Physiol Heart Circ Physiol 288: H1943-1954, 2005.

3. Baker AE, Dani R, Smith ER, Tyberg JV, and Belenkie I. Quantitative assessment of independent contributions of pericardium and septum to direct ventricular interaction. Am J Physiol 275: H476-483, 1998.

4. Baller D, Wolpers HG, Zipfel J, Bretschneider HJ, and Hellige G. Comparison of the effects of right atrial, right ventricular apex and atrioventricular sequential pacing on myocardial oxygen consumption and cardiac efficiency: A laboratory investigation. Pacing Clin Electrophysiol 11: 394-403, 1988.

5. Belenkie I, Dani R, Smith ER, and Tyberg JV. The importance of pericardial constraint in experimental pulmonary embolism and volume loading. Am Heart J 123: 733-742, 1992.

6. Belenkie I, Sas R, Mitchell J, Smith ER, and Tyberg JV. Opening the pericardium during pulmonary artery constriction improves cardiac function. J Appl Physiol 96: 917-922, 2004.

7. Beyar R. Heart inefficiency in pulmonary hypertension: A double jeopardy. J Am Coll Cardiol 51: 758-759, 2008.

8. Beyar R, Dong SJ, Smith ER, Belenkie I, and Tyberg JV. Ventricular interaction and septal deformation: A model compared with experimental data. Am J Physiol 265: H20442056, 1993.

9. Bishop JE, Rhodes S, Laurent GJ, Low RB, and Stirewalt WS. Increased collagen synthesis and decreased collagen degradation in right ventricular hypertrophy induced by pressure overload. Cardiovasc Res 28: 1581-1585, 1994.

10. Blanchard DG, and Dittrich HC. Pericardial adaptation in severe chronic pulmonary hypertension. An intraoperative transesophageal echocardiographic study. Circulation 85: 1414-1422, 1992.

11. Bleeker GB, Schalij MJ, Nihoyannopoulos P, Steendijk P, Molhoek SG, van Erven L, Bootsma M, Holman ER, van der Wall EE, and Bax JJ. Left ventricular dyssynchrony predicts right ventricular remodeling after cardiac resynchronization therapy. J Am Coll Cardiol 46: 2264-2269, 2005.

12. Bogaard HJ, Abe K, Vonk Noordegraaf A, and Voelkel NF. The right ventricle under pressure: Cellular and molecular mechanisms of right-heart failure in pulmonary hypertension. Chest 135: 794-804, 2009. 


\section{Chapter 8}

13. Bossone E, Avelar E, Bach DS, Gillespie B, Rubenfire M, and Armstrong WF. Diagnostic value of resting tricuspid regurgitation velocity and right ventricular ejection flow parameters for the detection of exercise induced pulmonary arterial hypertension. Int J Card Imaging 16: 429-436, 2000.

14. Brinker JA, Weiss JL, Lappe DL, Rabson JL, Summer WR, Permutt S, and Weisfeldt ML. Leftward septal displacement during right ventricular loading in man. Circulation 61: 626-633, 1980.

15. Calvin JE. Optimal right ventricular filling pressures and the role of pericardial constraint in right ventricular infarction in dogs. Circulation 84: 852-861, 1991.

16. Carere R, Patterson GA, Liu P, Williams T, Maurer J, and Grossman R. Right and left ventricular performance after single and double lung transplantation. The toronto lung transplant group. J Thorac Cardiovasc Surg 102: 115-122; discussion 122-113, 1991.

17. Chemla D, Castelain V, Herve P, Lecarpentier $Y$, and Brimioulle S. Haemodynamic evaluation of pulmonary hypertension. Eur Respir J 20: 1314-1331, 2002.

18. Chen PS, Moser KM, Dembitsky WP, Auger WR, Daily PO, Calisi CM, Jamieson SW, and Feld GK. Epicardial activation and repolarization patterns in patients with right ventricular hypertrophy. Circulation 83: 104-118, 1991.

19. Chin $\mathrm{KM}, \mathrm{Kim} \mathrm{NH}$, and Rubin LJ. The right ventricle in pulmonary hypertension. Coron Artery Dis 16: 13-18, 2005.

20. Dabestani A, Mahan G, Gardin JM, Takenaka K, Burn C, Allfie A, and Henry WL. Evaluation of pulmonary artery pressure and resistance by pulsed doppler echocardiography. Am J Cardiol 59: 662-668, 1987.

21. Dellegrottaglie S, Sanz J, Poon M, Viles-Gonzalez JF, Sulica R, Goyenechea M, Macaluso F, Fuster $V$, and Rajagopalan S. Pulmonary hypertension: Accuracy of detection with left ventricular septal-to-free wall curvature ratio measured at cardiac mr. Radiology 243: 63-69, 2007.

22. Dittrich HC, Chow LC, and Nicod PH. Early improvement in left ventricular diastolic function after relief of chronic right ventricular pressure overload. Circulation 80: 823830, 1989.

23. Dittrich HC, McCann HA, and Blanchard DG. Cardiac structure and function in chronic thromboembolic pulmonary hypertension. Am J Card Imaging 8: 18-27, 1994.

24. Doherty NE, 3rd, Fujita N, Caputo GR, and Higgins CB. Measurement of right ventricular mass in normal and dilated cardiomyopathic ventricles using cine magnetic resonance imaging. Am J Cardiol 69: 1223-1228, 1992.

25. Dong SJ, Crawley AP, MacGregor JH, Petrank YF, Bergman DW, Belenkie I, Smith ER, Tyberg JV, and Beyar R. Regional left ventricular systolic function in relation to the cavity geometry in patients with chronic right ventricular pressure overload. A three-dimensional tagged magnetic resonance imaging study. Circulation 91: 2359-2370, 1995. 
26. Dong SJ, Smith ER, and Tyberg JV. Changes in the radius of curvature of the ventricular septum at end diastole during pulmonary arterial and aortic constrictions in the dog. Circulation 86: 1280-1290, 1992.

27. Faber MJ, Dalinghaus M, Lankhuizen IM, Steendijk P, Hop WC, Schoemaker RG, Duncker DJ, Lamers JM, and Helbing WA. Right and left ventricular function after chronic pulmonary artery banding in rats assessed with biventricular pressure-volume loops. Am J Physiol Heart Circ Physiol 291: H1580-1586, 2006.

28. Freeman GL, and LeWinter MM. Pericardial adaptations during chronic cardiac dilation in dogs. Circ Res 54: 294-300, 1984.

29. Freeman $\mathrm{GL}$, and LeWinter MM. Role of parietal pericardium in acute, severe mitral regurgitation in dogs. Am J Cardiol 54: 217-219, 1984.

30. Fujii J, Yazaki Y, Sawada H, Aizawa T, Watanabe H, and Kato K. Noninvasive assessment of left and right ventricular filling in myocardial infarction with a two-dimensional doppler echocardiographic method. J Am Coll Cardiol 5: 1155-1160, 1985.

31. Galie N, Torbicki A, Barst R, Dartevelle P, Haworth S, Higenbottam T, Olschewski $H$, Peacock A, Pietra G, Rubin LJ, Simonneau G, Priori SG, Garcia MA, Blanc JJ, Budaj A, Cowie M, Dean V, Deckers J, Burgos EF, Lekakis J, Lindahl B, Mazzotta G, McGregor K, Morais J, Oto A, Smiseth OA, Barbera JA, Gibbs S, Hoeper M, Humbert M, Naeije R, and Pepke-Zaba J. Guidelines on diagnosis and treatment of pulmonary arterial hypertension. The task force on diagnosis and treatment of pulmonary arterial hypertension of the european society of cardiology. Eur Heart J 25: 2243-2278, 2004.

32. Gan CT, Lankhaar JW, Marcus JT, Westerhof N, Marques KM, Bronzwaer JG, Boonstra A, Postmus PE, and Vonk-Noordegraaf A. Impaired left ventricular filling due to right-to-left ventricular interaction in patients with pulmonary arterial hypertension. Am J Physiol Heart Circ Physiol 290: H1528-1533, 2006.

33. Gomez A, Bialostozky D, Zajarias A, Santos E, Palomar A, Martinez ML, and Sandoval J. Right ventricular ischemia in patients with primary pulmonary hypertension. J Am Coll Cardiol 38: 1137-1142, 2001.

34. Grines CL, Bashore TM, Boudoulas H, Olson S, Shafer P, and Wooley CF. Functional abnormalities in isolated left bundle branch block. The effect of interventricular asynchrony. Circulation 79: 845-853, 1989.

35. Gurudevan SV, Malouf PJ, Auger WR, Waltman TJ, Madani M, Raisinghani AB, DeMaria $A N$, and Blanchard DG. Abnormal left ventricular diastolic filling in chronic thromboembolic pulmonary hypertension: True diastolic dysfunction or left ventricular underfilling? J Am Coll Cardiol 49: 1334-1339, 2007.

36. Hajduczok ZD, Weiss RM, Stanford W, and Marcus ML. Determination of right ventricular mass in humans and dogs with ultrafast cardiac computed tomography. Circulation 82: 202-212, 1990. 


\section{Chapter 8}

37. Handoko ML, Lamberts RR, Redout EM, de Man FS, Boer C, Simonides WS, Paulus WJ, Westerhof N, Allaart CP, and Vonk-Noordegraaf A. Right ventricular pacing improves right heart function in experimental pulmonary arterial hypertension: A study in the isolated heart. Am J Physiol Heart Circ Physiol 2009.

38. Hardziyenka M, Campian ME, Bouma BJ, Linnenbank AC, de Bruin-Bon HA, Kloek JJ, van der Wal AC, Baan J, Jr., de Beaumont EM, Reesink HJ, de Bakker JM, Bresser P, and Tan HL. Right-to-left ventricular diastolic delay in chronic thromboembolic pulmonary hypertension is associated with activation delay and action potential prolongation in right ventricle. Circ Arrhythm Electrophysiol 2: 555-561, 2009.

39. Hirschfeld S, Meyer R, Schwartz DC, Korfhagen J, and Kaplan S. Measurement of right and left ventricular systolic time intervals by echocardiography. Circulation 51: 304-309, 1975.

40. Horan LG, Flowers NC, and Havelda CJ. Relation between right ventricular mass and cavity size: An analysis of 1500 human hearts. Circulation 64: 135-138, 1981.

41. Kaul S. The interventricular septum in health and disease. Am Heart J 112: 568-581, 1986.

42. Keogh AM, Mayer E, Benza RL, Corris P, Dartevelle PG, Frost AE, Kim NH, Lang IM, Pepke-Zaba J, and Sandoval J. Interventional and surgical modalities of treatment in pulmonary hypertension. J Am Coll Cardiol 54: S67-77, 2009.

43. Kerckhoffs RC, Lumens J, Vernooy K, Omens JH, Mulligan LJ, Delhaas T, Arts T, McCulloch AD, and Prinzen FW. Cardiac resynchronization: Insight from experimental and computational models. Prog Biophys Mol Biol 97: 543-561, 2008.

44. Kerckhoffs RC, Neal ML, Gu Q, Bassingthwaighte JB, Omens JH, and McCulloch AD. Coupling of a $3 \mathrm{~d}$ finite element model of cardiac ventricular mechanics to lumped systems models of the systemic and pulmonic circulation. Ann Biomed Eng 35: 1-18, 2007.

45. King ME, Braun H, Goldblatt A, Liberthson R, and Weyman AE. Interventricular septal configuration as a predictor of right ventricular systolic hypertension in children: A crosssectional echocardiographic study. Circulation 68: 68-75, 1983.

46. Kingma I, Tyberg JV, and Smith ER. Effects of diastolic transseptal pressure gradient on ventricular septal position and motion. Circulation 68: 1304-1314, 1983.

47. Kuehne T, Yilmaz S, Steendijk P, Moore P, Groenink M, Saaed M, Weber O, Higgins CB, Ewert P, Fleck E, Nagel E, Schulze-Neick I, and Lange P. Magnetic resonance imaging analysis of right ventricular pressure-volume loops: In vivo validation and clinical application in patients with pulmonary hypertension. Circulation 110: 2010-2016, 2004.

48. Kuijpers NH, Ten Eikelder HM, Bovendeerd PH, Verheule S, Arts T, and Hilbers PA. Mechanoelectric feedback as a trigger mechanism for cardiac electrical remodeling: A model study. Ann Biomed Eng 36: 1816-1835, 2008. 
49. Kuijpers NH, ten Eikelder HM, Bovendeerd PH, Verheule S, Arts T, and Hilbers PA. Mechanoelectric feedback leads to conduction slowing and block in acutely dilated atria: A modeling study of cardiac electromechanics. Am J Physiol Heart Circ Physiol 292: H2832-2853, 2007.

50. Lamberts RR, Caldenhoven E, Lansink M, Witte G, Vaessen RJ, St Cyr JA, and Stienen GJ. Preservation of diastolic function in monocrotaline-induced right ventricular hypertrophy in rats. Am J Physiol Heart Circ Physiol 293: H1869-1876, 2007.

51. Lamberts RR, Vaessen RJ, Westerhof N, and Stienen GJ. Right ventricular hypertrophy causes impairment of left ventricular diastolic function in the rat. Basic Res Cardiol 102: 19-27, 2007.

52. Lazar JM, Flores AR, Grandis DJ, Orie JE, and Schulman DS. Effects of chronic right ventricular pressure overload on left ventricular diastolic function. Am J Cardiol 72: 1179-1182, 1993.

53. Lee JK, Kodama I, Honjo H, Anno T, Kamiya K, and Toyama J. Stage-dependent changes in membrane currents in rats with monocrotaline-induced right ventricular hypertrophy. Am J Physiol 272: H2833-2842, 1997.

54. Leeuwenburgh BP, Helbing WA, Steendijk P, Schoof PH, and Baan J. Biventricular systolic function in young lambs subject to chronic systemic right ventricular pressure overload. Am J Physiol Heart Circ Physiol 281: H2697-2704, 2001.

55. Li GR, Lau CP, Leung TK, and Nattel S. Ionic current abnormalities associated with prolonged action potentials in cardiomyocytes from diseased human right ventricles. Heart Rhythm 1: 460-468, 2004.

56. Little WC, Reeves RC, Arciniegas J, Katholi RE, and Rogers EW. Mechanism of abnormal interventricular septal motion during delayed left ventricular activation. Circulation 65: 1486-1491, 1982.

57. Liu L, Tockman B, Girouard S, Pastore J, Walcott G, KenKnight B, and Spinelli J. Left ventricular resynchronization therapy in a canine model of left bundle branch block. Am J Physiol Heart Circ Physiol 282: H2238-2244, 2002.

58. Lopez-Candales A, Dohi K, Bazaz R, and Edelman K. Relation of right ventricular free wall mechanical delay to right ventricular dysfunction as determined by tissue doppler imaging. Am J Cardiol 96: 602-606, 2005.

59. Louie EK, Rich S, and Brundage BH. Doppler echocardiographic assessment of impaired left ventricular filling in patients with right ventricular pressure overload due to primary pulmonary hypertension. J Am Coll Cardiol 8: 1298-1306, 1986.

60. Lowes BD, Minobe W, Abraham WT, Rizeq MN, Bohlmeyer TJ, Quaife RA, Roden RL, Dutcher DL, Robertson AD, Voelkel NF, Badesch DB, Groves BM, Gilbert EM, and Bristow MR. Changes in gene expression in the intact human heart. Downregulation of alphamyosin heavy chain in hypertrophied, failing ventricular myocardium. J Clin Invest 100: 2315-2324, 1997. 


\section{Chapter 8}

61. Lumens J, Arts T, Broers B, Boomars KA, van Paassen P, Prinzen FW, and Delhaas T. Right ventricular free wall pacing improves cardiac pump function in severe pulmonary arterial hypertension: A computer simulation analysis. Am J Physiol Heart Circ Physiol 297: H2196-2205, 2009.

62. Lumens J, Arts T, and Delhaas T. Ventricular mechanical asynchrony in pulmonary arterial hypertension: A model study. In: Functional Imaging and Modeling of the Heart, edited by Ayache N, Delingette H, and Sermesant M. Nice, France: Springer Berlin / Heidelberg, 2009, p. 249-257.

63. Lumens J, Delhaas T, Kirn B, and Arts T. Modeling ventricular interaction: A multiscale approach from sarcomere mechanics to cardiovascular system hemodynamics. Pac Symp Biocomput 378-389, 2008.

64. Lumens J, Delhaas T, Kirn B, and Arts T. Three-wall segment (TriSeg) model describing mechanics and hemodynamics of ventricular interaction. Ann Biomed Eng 37: 22342255, 2009.

65. Lurz P, Nordmeyer J, Coats L, Taylor AM, Bonhoeffer P, and Schulze-Neick I. Immediate clinical and haemodynamic benefits of restoration of pulmonary valvar competence in patients with pulmonary hypertension. Heart 95: 646-650, 2009.

66. Lurz P, Puranik R, Nordmeyer J, Muthurangu V, Hansen MS, Schievano S, Marek J, Bonhoeffer $\mathrm{P}$, and Taylor AM. Improvement in left ventricular filling properties after relief of right ventricle to pulmonary artery conduit obstruction: Contribution of septal motion and interventricular mechanical delay. Eur Heart J 30: 2266-2274, 2009.

67. Mahmud E, Raisinghani A, Hassankhani A, Sadeghi HM, Strachan GM, Auger W, DeMaria AN, and Blanchard DG. Correlation of left ventricular diastolic filling characteristics with right ventricular overload and pulmonary artery pressure in chronic thromboembolic pulmonary hypertension. J Am Coll Cardiol 40: 318-324, 2002.

68. Mangano DT, Van Dyke DC, Hickey RF, and Ellis RJ. Significance of the pericardium in human subjects: Effects on left ventricular volume, pressure and ejection. J Am Coll Cardiol 6: 290-295, 1985.

69. Marcus JT, Gan CT, Zwanenburg JJ, Boonstra A, Allaart CP, Gotte MJ, and VonkNoordegraaf A. Interventricular mechanical asynchrony in pulmonary arterial hypertension: Left-to-right delay in peak shortening is related to right ventricular overload and left ventricular underfilling. J Am Coll Cardiol 51: 750-757, 2008.

70. Marcus JT, Vonk Noordegraaf A, Roeleveld RJ, Postmus PE, Heethaar RM, Van Rossum $A C$, and Boonstra A. Impaired left ventricular filling due to right ventricular pressure overload in primary pulmonary hypertension: Noninvasive monitoring using MRI. Chest 119: 1761-1765, 2001. 
71. Menzel T, Kramm T, Mohr-Kahaly S, Mayer E, Oelert H, and Meyer J. Assessment of cardiac performance using tei indices in patients undergoing pulmonary thromboendarterectomy. Ann Thorac Surg 73: 762-766, 2002.

72. Menzel T, Wagner S, Kramm T, Mohr-Kahaly S, Mayer E, Braeuninger S, and Meyer J. Pathophysiology of impaired right and left ventricular function in chronic embolic pulmonary hypertension: Changes after pulmonary thromboendarterectomy. Chest 118 : 897-903, 2000.

73. Morris-Thurgood JA, and Frenneaux MP. Diastolic ventricular interaction and ventricular diastolic filling. Heart Fail Rev 5: 307-323, 2000.

74. Moustapha A, Kaushik V, Diaz S, Kang SH, and Barasch E. Echocardiographic evaluation of left-ventricular diastolic function in patients with chronic pulmonary hypertension. Cardiology 95: 96-100, 2001.

75. Olivetti G, Ricci R, Lagrasta C, Maniga E, Sonnenblick EH, and Anversa P. Cellular basis of wall remodeling in long-term pressure overload-induced right ventricular hypertrophy in rats. Circ Res 63: 648-657, 1988.

76. Olsen CO, Tyson GS, Maier GW, Spratt JA, Davis JW, and Rankin JS. Dynamic ventricular interaction in the conscious dog. Circ Res 52: 85-104, 1983.

77. Pitzalis MV, lacoviello M, Romito R, Guida P, De Tommasi E, Luzzi G, Anaclerio M, Forleo C, and Rizzon P. Ventricular asynchrony predicts a better outcome in patients with chronic heart failure receiving cardiac resynchronization therapy. J Am Coll Cardiol 45: 65-69, 2005.

78. Prinzen FW, Hunter WC, Wyman BT, and McVeigh ER. Mapping of regional myocardial strain and work during ventricular pacing: Experimental study using magnetic resonance imaging tagging. J Am Coll Cardiol 33: 1735-1742, 1999.

79. Reisner SA, Azzam Z, Halmann M, Rinkevich D, Sideman S, Markiewicz W, and Beyar R. Septal/free wall curvature ratio: A noninvasive index of pulmonary arterial pressure. J Am Soc Echocardiogr 7: 27-35, 1994.

80. Roeleveld RJ, Marcus JT, Faes TJ, Gan TJ, Boonstra A, Postmus PE, and VonkNoordegraaf A. Interventricular septal configuration at $\mathrm{mr}$ imaging and pulmonary arterial pressure in pulmonary hypertension. Radiology 234: 710-717, 2005.

81. Sadeghi HM, Kimura BJ, Raisinghani A, Blanchard DG, Mahmud E, Fedullo PF, Jamieson SW, and DeMaria AN. Does lowering pulmonary arterial pressure eliminate severe functional tricuspid regurgitation? Insights from pulmonary thromboendarterectomy. J Am Coll Cardiol 44: 126-132, 2004.

82. Sandoval J, Bauerle O, Palomar A, Gomez A, Martinez-Guerra ML, Beltran M, and Guerrero ML. Survival in primary pulmonary hypertension. Validation of a prognostic equation. Circulation 89: 1733-1744, 1994. 


\section{Chapter 8}

83. Schmitt B, Steendijk P, Lunze K, Ovroutski S, Falkenberg J, Rahmanzadeh P, Maarouf N, Ewert $P$, Berger $F$, and Kuehne T. Integrated assessment of diastolic and systolic ventricular function using diagnostic cardiac magnetic resonance catheterization: Validation in pigs and application in a clinical pilot study. JACC Cardiovasc Imaging 2: 1271-1281, 2009.

84. Sechtem U, Pflugfelder PW, Gould RG, Cassidy MM, and Higgins CB. Measurement of right and left ventricular volumes in healthy individuals with cine mr imaging. Radiology 163: 697-702, 1987.

85. Slinker BK, and Glantz SA. End-systolic and end-diastolic ventricular interaction. Am J Physiol 251: H1062-1075, 1986.

86. Stojnic BB, Brecker SJ, Xiao HB, Helmy SM, Mbaissouroum M, and Gibson DG. Left ventricular filling characteristics in pulmonary hypertension: A new mode of ventricular interaction. Br Heart J 68: 16-20, 1992.

87. Stolzmann P, Scheffel H, Leschka S, Schertler T, Frauenfelder T, Kaufmann PA, Marincek $\mathrm{B}$, and Alkadhi $\mathrm{H}$. Reference values for quantitative left ventricular and left atrial measurements in cardiac computed tomography. Eur Radiol 18: 1625-1634, 2008.

88. Streeter DD, Jr. Gross morphology and fiber geometry of the heart. In: Handbook of physiology, edited by Berne RM, Sperelakis N, and Geiger SR. Bethesda: American Physiological Society, 1979, p. 61-112.

89. Suga H, Hayashi T, and Shirahata M. Ventricular systolic pressure-volume area as predictor of cardiac oxygen consumption. Am J Physiol 240: H39-44, 1981.

90. Tanaka H, Tei C, Nakao S, Tahara M, Sakurai S, Kashima T, and Kanehisa T. Diastolic bulging of the interventricular septum toward the left ventricle. An echocardiographic manifestation of negative interventricular pressure gradient between left and right ventricles during diastole. Circulation 62: 558-563, 1980

91. Tei C, Dujardin KS, Hodge DO, Bailey KR, McGoon MD, Tajik AJ, and Seward SB. Doppler echocardiographic index for assessment of global right ventricular function. J Am Soc Echocardiogr 9: 838-847, 1996.

92. Tei C, Dujardin KS, Hodge DO, Kyle RA, Tajik AJ, and Seward JB. Doppler index combining systolic and diastolic myocardial performance: Clinical value in cardiac amyloidosis. J Am Coll Cardiol 28: 658-664, 1996.

93. ter Keurs HE, Rijnsburger $\mathbf{W H}$, van Heuningen R, and Nagelsmit MJ. Tension development and sarcomere length in rat cardiac trabeculae. Evidence of lengthdependent activation. Circ Res 46: 703-714, 1980.

94. van Wolferen SA, Marcus JT, Westerhof N, Spreeuwenberg MD, Marques KM, Bronzwaer JG, Henkens IR, Gan CT, Boonstra A, Postmus PE, and Vonk-Noordegraaf A. Right coronary artery flow impairment in patients with pulmonary hypertension. Eur Heart J 29: 120-127, 2008. 
95. Verbeek XA, Vernooy K, Peschar M, Van Der Nagel T, Van Hunnik A, and Prinzen FW. Quantification of interventricular asynchrony during lbbb and ventricular pacing. Am J Physiol Heart Circ Physiol 283: H1370-1378, 2002.

96. Vonk-Noordegraaf A, Marcus JT, Gan CT, Boonstra A, and Postmus PE. Interventricular mechanical asynchrony due to right ventricular pressure overload in pulmonary hypertension plays an important role in impaired left ventricular filling. Chest 128: 6285630S, 2005.

97. Watkins MW, and LeWinter MM. Physiologic role of the normal pericardium. Annu Rev Med 44: 171-180, 1993.

98. Weber KT, Janicki JS, Shroff S, and Fishman AP. Contractile mechanics and interaction of the right and left ventricles. Am J Cardiol 47: 686-695, 1981.

99. Yeo TC, Dujardin KS, Tei C, Mahoney DW, McGoon MD, and Seward JB. Value of a doppler-derived index combining systolic and diastolic time intervals in predicting outcome in primary pulmonary hypertension. Am J Cardiol 81: 1157-1161, 1998.

100. Zong P, Tune JD, and Downey HF. Mechanisms of oxygen demand/supply balance in the right ventricle. Exp Biol Med (Maywood) 230: 507-519, 2005. 

Summary

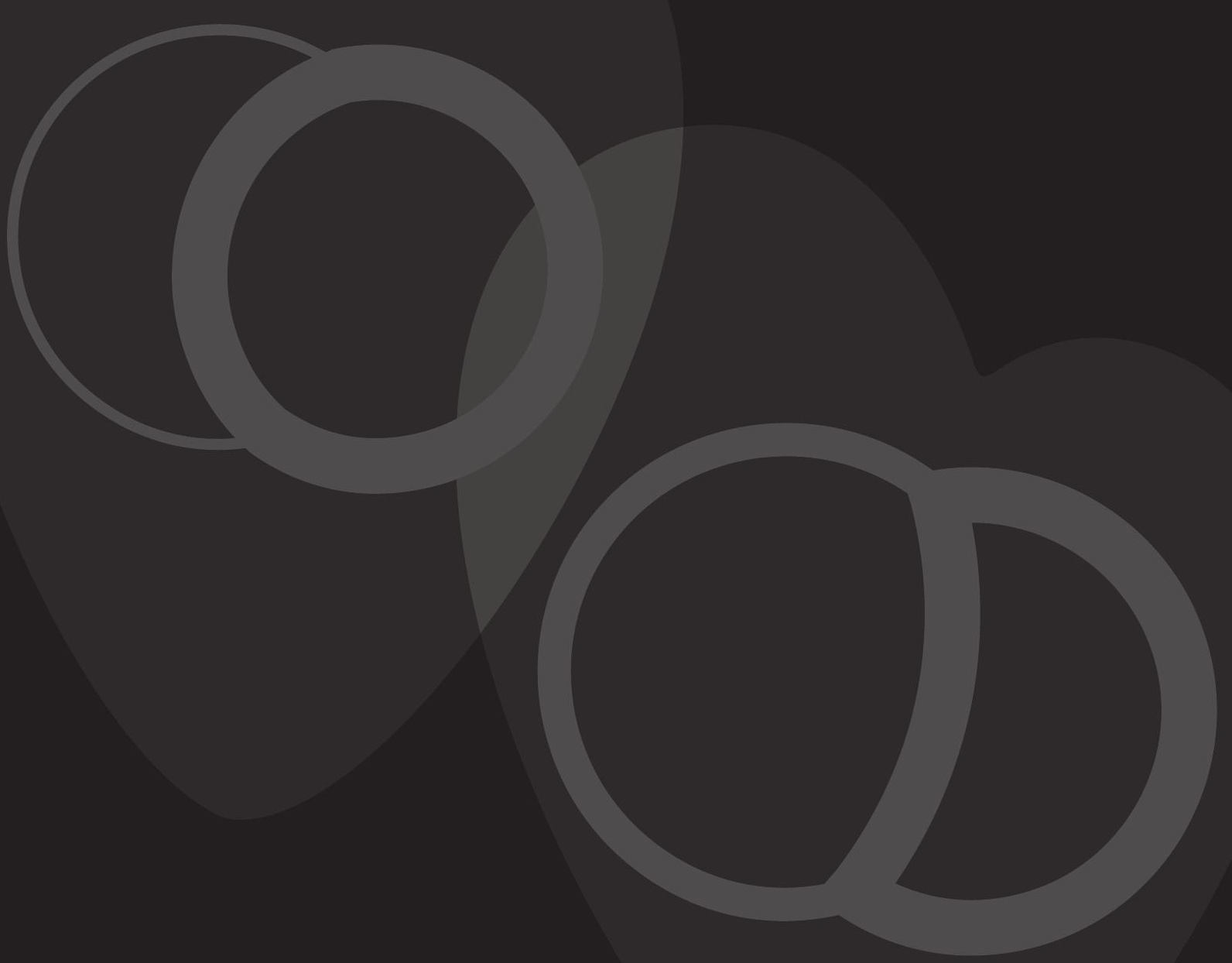


Pulmonary hypertension $(\mathrm{PH})$ is characterized by elevated blood pressure in the pulmonary artery. The clinical classification of PH distinguishes five subgroups. Each subgroup shares pathological and clinical features as well as similar therapeutic options. In this thesis, we focus on pulmonary arterial hypertension (PAH) and chronic thromboembolic pulmonary hypertension (CTEPH), both resulting from elevated vascular resistance on the arterial side of the pulmonary circulation. PAH is characterized by progressive structural remodeling of the pulmonary arterioles. In CTEPH, pulmonary vascular resistance is elevated due to thrombi and resistive lesions in the more proximal pulmonary arteries. Unless stated differently, CTEPH is considered as a type of PAH in the remainder of this summary, since it also progressively develops due to similar histopathological changes in the pulmonary vasculature.

$\mathrm{PAH}$ is associated with chronic elevation of mechanical pump load of the right ventricle. When left untreated, PAH eventually leads to right heart failure and premature death. The underlying mechanisms by which PAH leads to heart failure are poorly understood. In this thesis, we elucidate the mechanisms by which PAH affects left ventricular (LV) and right ventricular (RV) pump function. These new insights are believed to be essential for improvement of therapy and diagnostics in patients with PAH. The general aim of this thesis as well as the specific aims of the separate studies, as included in this thesis, are briefly introduced in chapter 1.

In the human heart, the left ventricle (LV) and the right ventricle (RV) are functionally dependent due to mechanical ventricular interaction via the interventricular septum and due to hemodynamic ventricular interaction since RV outflow determines LV inflow. Consequently, PAH affects both ventricles as mentioned in chapter 2. A novel mathematical model of ventricular mechanics and hemodynamics (TriSeg model), incorporating ventricular interaction, has been developed and used for quantitative assessment of ventricular pump function under normal and PAH conditions.

In chapters 3 and 4 , we present the theory behind the TriSeg model describing ventricular mechanics and hemodynamics incorporating mechanical interaction of the ventricular walls. Three wall segments representing the LV free wall, the interventricular septum, and the RV free wall are mechanically coupled, as expressed by the equilibrium of tensile forces in their junction. Global LV and $\mathrm{RV}$ pump mechanics are related to representative myofiber mechanics in the three ventricular walls, using prominently the principle of conservation of energy. Hemodynamic ventricular interaction is achieved by implementation of the TriSeg model in the modular CircAdapt model describing mechanics and 
hemodynamics of the whole human circulation. The combined CircAdapt/TriSeg model allows realistic beat-to-beat simulation of time-dependent ventricular mechanics and hemodynamics such as volumes and pressures of the cardiac cavities, geometries and myofiber mechanics of the cardiac walls, and flows through the valves. Furthermore, the model allows adaptation of size and mass of cardiac walls and large blood vessels to normalize local mechanical load to tissue-specific physiological levels.

In chapter 5, severely decompensated PAH is simulated by increasing RV pressure load without adaptation. This simulation shows many similarities with data obtained in patients with severe PAH, e.g., RV dilatation, leftward septal bulging, and delayed peak shortening of the RV free wall as compared to that of the LV free wall and septum. Myofiber work in the RV free wall was three times higher than that in the septum and the LV free wall. In our model, we tested the hypothesis that RV resynchronization therapy improves ventricular mechanical synchrony and, thereby, RV pump function in PAH. Our model simulations show that preexcitation of the RV free wall relieves the RV free wall from the exorbitantly high myofiber work at the cost of an increase of myofiber work in the LV free wall and septum. Apparently, the mechanical interaction of the ventricular walls allowed redistribution of myofiber work over the ventricular walls. Upon 40-ms preexcitation of the RV free wall, mechanical load appeared most homogeneously distributed over the ventricular walls, RV and LV pressure curves were synchronized, and RV pump function was moderately improved. The latter improvement was evidenced by increase of $\mathrm{RV} d P / \mathrm{dt}_{\max }$, decrease of RV end-diastolic pressure, and decrease of RV end-diastolic volume. These simulations suggest that early pacing of the RV free wall is a potential treatment of RV failure in patients with severe PAH.

In patients with chronic thromboembolic pulmonary hypertension (CTEPH), chronic increase of RV afterload is associated with an abnormal LV filling pattern, i.e., peak blood flow velocity of the early mitral filling wave (E-velocity) is smaller than that of the atrial contraction-induced late filling wave (Avelocity). Pulmonary thromboendarterectomy (PTE) is a surgical intervention that acutely reduces pulmonary vascular resistance and that is associated with acute increase of mitral E/A-ratio. Abolishment of leftward septal bulging and increase in RV systolic output are the two mechanisms that are thought to be responsible for the increase of the E/A-ratio with PTE. In chapter 6, the TriSeg model is used to estimate the separate contributions of both mechanisms in a group of CTEPH patients undergoing PTE. The effect of PTE on LV filling hemodynamics as predicted by our model is surprisingly similar to the effect as observed in 
patients. Mitral E/A, mitral E-velocity, LV end-diastolic volume, and septal curvature increased from subnormal values in CTEPH before PTE to normal values after PTE, whereas RV end-diastolic volume decreased to normal value after PTE. Effects of RV pressure overload and of RV systolic output on LV filling hemodynamics are separated quantitatively by simulating either an exclusive decrease of mean pulmonary artery pressure or an exclusive increase of cardiac output, respectively. With exclusive decrease of mean pulmonary artery pressure, mitral E/A-ratio increased 26\%, LV filling pressure decreased 16\%, and leftward septal bulging was abolished. With exclusive increase of cardiac output, mitral E/A-ratio increased 53\%, LV filling pressure increased $62 \%$, whereas leftward septal bulging persisted. These data show that increase of mitral E/A-ratio with PTE in CTEPH patients is largely the effect of increased RV systolic output (hemodynamic ventricular interaction). Abolishment of leftward septal bulging (mechanical ventricular interaction) appears to be of less importance.

Finally, a novel patient-specific simulation procedure is presented in chapter 7 . With this procedure, ventricular mechanics is assessed in a heterogeneous patient group consisting of PAH and CTEPH patients with and without right bundle branch block (RBBB). Patient-specific simulations showed ventricular mechanical asynchrony in all patients. This asynchrony was evidenced by delay of peak shortening of the RV free wall and by early diastolic lengthening of the septum. Comparison of measured and simulated ventricular strain patterns revealed a systematic underestimation of early diastolic lengthening of the septum in the simulations. Moreover, the underestimation was more pronounced in the simulations of patients with higher amplitude of early diastolic septal lengthening. This discrepancy between measurements and simulations may be explained by the fact that our sarcomere contraction model, as implemented in the TriSeg model, is based on experimental data obtained from isolated cardiac muscle under normal physiological loading conditions. In our PAH patients, however, the chronic increase of mechanical load of the RV myocardium likely modifies myocardial contraction. Nonetheless, most of our patient-specific simulations suggest that the ventricular mechanical asynchrony in PAH can largely be explained using regular mechanical properties of the myocardial tissue. In a subgroup of patients with RBBB, agreement between measured and simulated ventricular mechanical asynchrony was further improved when the onset of mechanical activation of the RV free wall was delayed, also resulting in delay of mechanical relaxation. These findings suggest that, in PAH, ventricular mechanical asynchrony is caused by delay of mechanical relaxation of the RV free wall. Furthermore, it has been shown that the asynchrony occurred irrespective whether the delay of 
mechanical relaxation of the RV free wall was caused by prolonged mechanical activation of the RV free wall, delayed onset of activation, or both.

In conclusion, we presented a novel mathematical model of ventricular mechanics and hemodynamics, incorporating mechanical ventricular interaction. This model has been shown to be a valuable simulation tool for elucidating mechanisms of cardiac pathophysiology related to PAH and for patient-specific assessment of ventricular mechanics and hemodynamics in patients with PAH. Each of the studies presented in this thesis starts with fitting the model to the average of a set of clinical measurements obtained in patients with PAH in order to obtain a representative pathology-specific simulation. Using such pathologyspecific simulation as point of departure, it is shown in our simulations that:

1. preexcitation of the RV free wall acutely improves RV pump function in $\mathrm{PAH}$ and, hence, RV pacing may be a potential treatment of RV failure in patients with severe PAH,

2. LV underfilling and not leftward septal bulging dominates abnormal LV filling hemodynamics in patients with CTEPH, and

3. ventricular mechanical asynchrony, as often present in PAH patients, is caused by delay of mechanical relaxation of the RV free wall compared to that of the LV free wall and the septum. 

Samenvatting 
Pulmonale hypertensie (PH) is de gemeenschappelijke naam voor een aantal aandoeningen die alle gekenmerkt worden door verhoogde bloeddruk in de longslagader (arteria pulmonalis). In de kliniek wordt op basis van pathologische, klinische en therapeutische criteria onderscheid gemaakt tussen vijf hoofdgroepen van $\mathrm{PH}$. Het onderzoek in dit proefschrift richt zich op PH als gevolg van een verhoogde weerstand van de bloedvaten aan de slagaderlijke (arteriële) zijde van de longcirculatie. Dit is het geval bij pulmonale arteriële hypertensie (PAH) en chronische trombo-embolische pulmonale hypertensie (CTEPH). PAH wordt gekenmerkt door progressieve structurele remodellering van de pulmonale arteriolen. In het geval van CTEPH is de pulmonale vaatweerstand verhoogd als gevolg van trombi en vernauwende laesies in de proximale arteriën van de pulmonale circulatie. In het restant van deze samenvatting wordt CTEPH onder een noemer gebracht met PAH, tenzij anders vermeld.

De drukoverbelasting als gevolg van PAH leidt tot een verhoging van de pompbelasting van het rechter ventrikel van het hart. Zonder behandeling zal dit uiteindelijk leiden tot rechter hartfalen en vroegtijdige dood. De hedendaagse kennis van mechanismen die ten grondslag liggen aan hartfalen als gevolg van PAH is beperkt. Het onderzoek zoals beschreven in dit proefschrift, heeft als doel nieuw inzicht te verschaffen in de invloed van PAH op linker ventrikel (LV) en rechter ventrikel (RV) pompfunctie. Deze nieuwe inzichten zijn van essentieel belang voor verbetering van zowel therapie als diagnostiek in patiënten met $\mathrm{PAH}$. In hoofdstuk 1 zijn het algemene doel en de specifieke vraagstellingen van de afzonderlijke hoofdstukken van dit proefschrift kort uiteengezet.

In het menselijke hart zijn het linker en het rechter ventrikel functioneel afhankelijk van elkaar, enerzijds ten gevolge van mechanische interactie via het interventriculaire septum, anderzijds ten gevolge van hemodynamische interactie via de pulmonale en de systemische circulatie. In Hoofdstuk 2 wordt beschreven hoe deze ventriculaire interactie ervoor zorgt dat PAH de pompfunctie van beide ventrikels beïnvloedt. Voor kwantitatieve beschrijving van ventriculaire pompfunctie zowel onder normale als onder PAH condities is een nieuw wiskundig model ontwikkeld (TriSeg model), dat de mechanica en hemodynamica van de ventrikels beschrijft inclusief de mechanische interactie tussen beide ventrikels. In hoofdstukken 3 en 4 wordt de theorie beschreven die ten grondslag ligt aan het TriSeg model. In dit model worden de LV vrije wand, het interventriculaire septum en de RV vrije wand gerepresenteerd door drie afzonderlijke wandsegmenten die mechanisch gekoppeld zijn. Deze koppeling is wiskundig beschreven door een krachtenbalans. De relatie tussen de mechanica van globale ventriculaire pompfunctie en de mechanica van de 
spiervezels in de wanden van de ventrikels wordt beschreven op basis van energiebehoud. Hemodynamische interactie tussen beide ventrikels is tot stand gebracht door integratie van het TriSeg model in het modulaire CircAdapt model, dat de mechanica en hemodynamica van het gehele menselijke cardiovasculaire systeem beschrijft. Het gecombineerde CircAdapt/TriSeg model maakt het mogelijk om de mechanica en de hemodynamica van de ventrikels realistisch en slag-op-slag te simuleren. Voorbeelden van beschikbare gesimuleerde grootheden zijn: ventriculaire holtevolumina en drukken, geometrie van de ventriculaire wanden, mechanica van de spiervezels in de ventriculaire wanden en stroming van bloed door de hartkleppen. Het model biedt de mogelijkheid tot simulatie van structurele adaptatie van het hart en de grote bloedvaten aan de mechanische belasting. Grootte en massa van hartspierwanden en bloedvaten worden aangepast zodat de lokale mechanische belasting van het weefsel in overeenstemming is met weefselspecifieke fysiologische normaalwaarden.

In hoofdstuk 5 is ernstig gedecompenseerde PAH gesimuleerd door in het model de drukbelasting van het rechter ventrikel te verhogen zonder adaptatie toe te staan. De resulterende simulatie toont de volgende gelijkenissen met data die verkregen zijn in patiënten met ernstige PAH: RV dilatatie, linkswaartse buiging van het septum, relatieve vertraging van piekverkorting van de RV vrije wand ten opzichte van die van de LV vrije wand en het septum. In de simulatie is de spiervezelarbeid per hartslag drie keer groter in de RV vrije wand dan in de LV vrije wand en in het septum. Op basis van deze resultaten is de hypothese geformuleerd dat in patiënten met PAH vervroegde activatie van de RV vrije wand de ventriculaire mechanische synchronie herstelt en daardoor ook de RV pompfunctie verbetert. Onze simulaties tonen aan dat vervroegde activatie van de RV vrije wand in PAH zorgt voor een verlaging van de buitensporig hoge spiervezelarbeid in de RV vrije wand en voor een verhoging van de arbeid die verricht wordt door de spiervezels in de LV vrije wand en in het septum. Deze redistributie van spiervezelarbeid wordt mogelijk gemaakt door de mechanische koppeling van de ventriculaire wanden. Wanneer de RV vrije wand 40 ms eerder geactiveerd wordt dan de LV vrije wand en het septum, is de mechanische belasting het meest homogeen verdeeld over de ventriculaire wanden, zijn de RV en de LV drukcurven synchroon en is de RV pompfunctie verbeterd. Deze laatste verbetering komt tot uiting door een toename van $\mathrm{RV} d P / \mathrm{dt}_{\max }$, door afname van $\mathrm{RV}$ eind-diastolische druk en door afname van RV eind-diastolisch volume. Onze simulaties suggereren dat vervroegde activatie van de RV vrije wand mogelijk gebruikt kan worden voor behandeling van rechter hartfalen in patiënten met ernstige PAH. 
In patiënten met chronische trombo-embolische pulmonale hypertensie (CTEPH) gaat de chronische verhoging van RV afterload gepaard met een abnormaal LV vullingpatroon: piek bloedstroomsnelheid door de mitralisklep tijdens de vroege passieve vullingfase (E-snelheid) is kleiner dan die tijdens de late actieve vullingfase (A-snelheid). Deze actieve vullingfase wordt geïnduceerd door contractie van het linker atrium. CTEPH patienten kunnen behandeld worden met pulmonale trombo-endarteriëctomie (PTE). Tijdens deze ingreep worden trombi en vernauwende laesies in het pulmonale vaatbed waar mogelijk chirurgisch verwijderd. PTE resulteert in de meeste gevallen in een acute reductie van de pulmonale vaatweerstand (en dus ook van RV druk) en een acute verhoging van de mitralis E/A-ratio (E/A). De daling van $R V$ druk als gevolg van PTE zorgt voor een verandering van de beweging van het septum, namelijk van abnormale linkswaartse buiging voor PTE naar normale rechtswaartse buiging na PTE. De stijging van E/A met PTE kan verklaard worden aan de hand van twee mechanismen, namelijk opheffing van het naar links doorbuigen van het septum en toename van RV systolische output. Het TriSeg model is in hoofdstuk 6 gebruikt om de afzonderlijke bijdragen van beide mechanismen te kwantificeren in een groep patiënten met CTEPH die PTE ondergaan. Het effect van PTE op LV vulling hemodynamica zoals het door ons model wordt voorspeld, is kwantitatief in overeenstemming met het effect zoals gemeten in patiënten. Zo voorspelt ons model dat E/A, E-snelheid en LV eind-diastolisch volume stijgen van subnormale waarden in CTEPH naar normale waarden na PTE. Bovendien normaliseert de kromming van het septum (van linkswaarts naar rechtswaarts) en daalt RV eind-diastolisch volume naar een normale waarde na simulatie van PTE. De afzonderlijke effecten van RV drukbelasting en van RV systolische output op de vulling hemodynamica van de LV zijn met behulp van het model kwantitatief gescheiden door respectievelijk alleen een afname van gemiddelde bloeddruk in de arteria pulmonalis ofwel alleen een toename van cardiac output te simuleren. Met enkel afname van de gemiddelde druk in de arteria pulmonalis neemt E/A $26 \%$ toe, neemt LV vullingdruk met $16 \%$ af en verdwijnt de linkswaartse buiging van het septum. Met enkel toename van cardiac output nemen E/A en LV vullingdruk met respectievelijk $53 \%$ en $62 \%$ toe, terwijl de linkswaartse buiging van het septum persisteert. Deze data suggereren dat de toename van E/A na behandeling met PTE van patiënten met CTEPH voornamelijk het gevolg is van toename van RV systolische output (hemodynamische ventriculaire interactie). Opheffing van de linkswaartse buiging van het septum (mechanische ventriculaire interactie) lijkt een minder belangrijke rol te spelen in de normalisatie van het LV vullingpatroon. 


\section{Samenvatting}

Tenslotte is in hoofdstuk 7 de ontwikkeling van een nieuwe patiënt-specifieke simulatiemethode beschreven. Deze methode is gebruikt voor kwantitatieve benadering van ventriculaire mechanica in een studiegroep bestaande uit patiënten met pulmonale hypertensie van verschillende etiologie (PAH of CTEPH) met of zonder rechter bundeltakblok (RBTB). De patiënt-specifieke simulaties vertonen ventriculaire mechanische asynchronie in alle patiënten. Deze asynchronie wordt gekenmerkt door vertraging van piek verkorting van de RV vrije wand en door vroegtijdige verlenging van het septum aan het begin van LV diastole. Een vergelijking van gesimuleerde met gemeten ventriculaire strainpatronen heeft onthuld dat het model de vroegtijdige diastolische verlenging van het septum systematisch onderschat. Bovendien bleek deze onderschatting relatief groter te zijn in simulaties van patiënten met een grotere amplitude van vroegtijdige diastolische verlenging van het septum. Een mogelijke verklaring voor deze discrepantie tussen meting en simulatie kan liggen in het feit dat het contractiemodel van de sarcomeer in het TriSeg model gebaseerd is op experimentele data die verkregen zijn in geïsoleerde hartspiercellen onder fysiologische mechanische belasting. Echter, in PAH-patiënten is de mechanische belasting van het RV hartspierweefsel zeer waarschijnlijk chronisch verhoogd en gelden mogelijk andere contractiemodellen. Desalniettemin wekken onze patiënt-specifieke simulaties sterk de indruk dat de ventriculaire mechanische asynchronie in PAH gedeeltelijk verklaard kan worden aan de hand van fysiologische mechanische eigenschappen van het hartspierweefsel. Door de start van mechanische activatie (en dus ook de relaxatie) van de $R V$ vrije wand te vertragen is in een subgroep van patiënten met RBTB de gesimuleerde ventriculaire mechanische asynchronie in kwantitatieve overeenstemming gebracht met de gemeten asynchronie. Deze bevinding suggereert dat de ventriculaire mechanische asynchronie in PAH veroorzaakt wordt door vertraging van mechanische relaxatie van de RV vrije wand ten opzicht van die van de LV vrije wand en het septum. Onze simulaties tonen ook aan dat asynchronie optreedt ongeacht de exacte oorzaak van de vertraging van de mechanische relaxatie van de RV vrije wand. Zowel een verlengde duur van mechanische activatie als een vertraagde start van activatie of zelfs beide effecten tegelijkertijd kunnen de mechanische asynchronie veroorzaken.

Samenvattend is in dit proefschrift een nieuw wiskundig model gepresenteerd, dat de mechanica en hemodynamica van de ventrikels beschrijft inclusief de mechanische interactie tussen de ventriculaire wanden. Verder hebben we laten zien dat het model enerzijds nuttig kan zijn voor fundamenteel onderzoek naar mechanismen van cardiale pathofysiologie in PAH en anderzijds voor patiëntspecifieke benadering van ventriculaire mechanica en hemodynamica in patiënten 
met PAH. Elke afzonderlijke studie van dit proefschrift begint met het in overeenstemming brengen van het model met de gemiddelde waarden van een set klinische metingen verkregen in patiënten met PAH. Op die manier wordt voor elke studiegroep een representatieve pathologie-specifieke simulatie verkregen. Startende vanuit een dergelijke pathologie-specifieke simulatie is aan de hand van simulaties aangetoond dat:

1. vervroegde activatie van de RV vrije wand resulteert in een acute verbetering van RV pompfunctie in PAH en dus mogelijk gebruikt kan worden voor behandeling van rechter hartfalen in patiënten met ernstige PAH;

2. vooral LV ondervulling en in mindere mate de linkswaartse buiging van het septum zorgt voor een abnormaal LV vullingpatroon in patiënten met CTEPH; en

3. ventriculaire mechanische asynchronie in PAH veroorzaakt wordt door vertraging van mechanische relaxatie van de RV vrije wand ten opzichte van die van de LV vrije wand en het septum. 



\section{Dankwoord}


Dankwoord 
Dit proefschrift is tot stand gekomen mede door de inspanning en kennis van vele anderen. Daarom zou ik graag willen besluiten met een woord van dank gericht tot iedereen die een bijdrage heeft geleverd, in welke vorm dan ook. Een aantal mensen wil ik in het bijzonder bedanken.

Allereerst zijn dat mijn promotores Prof. dr. ir. Theo Arts en Prof. dr. Tammo Delhaas. Beste Theo en Tammo, telkens wanneer iemand mij vroeg wie mijn begeleiders waren, heb ik jullie in één adem genoemd. Voor mij vormden jullie het ideale begeleidingsduo. De combinatie van Theo's theoretische kennis en Tammo's oog voor de klinische/fysiologische toepassing vormt een solide basis voor een medisch ingenieur die graag wil promoveren. Vooral jullie directe manier van communiceren en bekritiseren heeft mij gevormd tot de wetenschapper die ik nu ben. Wilco en ik hebben jullie niet voor niets omgedoopt tot "TNT...explosief en efficiënt"! Ontzettend bedankt voor het feit dat jullie mij tijdens mijn afstuderen gevraagd hebben om promotieonderzoek bij jullie te doen.

Beste Theo, jij was voor mij de perfecte mentor. Jouw "double-bubble" ideeën vormen het skelet van mijn proefschrift. Het maakte niet uit waar je je bevond (UM, Eijsden of Vallandry), ik wist dat ik altijd bij je kon aankloppen voor hulp. Zelfs toen ik als BMT-student stage liep in Auckland, heb je mij op afstand van de nodige begeleiding voorzien. Op momenten dat ik alleen maar problemen zag, heb jij mij elke keer weer met veel overtuiging laten inzien dat bepaalde onderzoeksresultaten positief benaderd kunnen worden. Op die manier heb je mij geholpen de gebruikelijke AlO-dip te overleven. Je vage stelligheid kon mij soms goed de keel uit hangen, maar diende tevens als trigger om dingen tot op de bodem uit te zoeken. Tot mijn grote frustratie bleek je uiteindelijk bijna altijd gelijk te hebben. Toch ben ik blij dat je, mede dankzij ons (Tammo, Wilco en mij), bepaalde dingen door een andere bril bent gaan bekijken, hoewel Jacqueline daar (naar jouw zeggen) niet zo blij mee was. Wat heb ik genoten van onze retraiteweken in Vallandry, ontzettend bedankt daarvoor!

Beste Tammo, ik zal onze eerste ontmoeting op jouw oude kamer in het Dr. Tansgebouw nooit vergeten. Het enthousiasme waarmee je over je onderzoek praatte, was nog groter dan de bende op je kamer. Jij hebt vanaf dat moment mijn interesse voor hartmechanica weten te wekken. Het was vooral de ongedwongen sfeer, die jij vanaf dat eerste moment elke keer weer wist te creëren, die mij enorm aansprak. Na of tijdens het werk was er altijd tijd voor een gezellig "YouTube-momentje" of een borrel. Je was werkelijk 24 uur per dag bereikbaar voor hulp en nooit te beroerd om acuut iets voor mij te regelen. 
Ik kijk met veel plezier terug naar de talloze geplande en spontane etentjes waarvan Irene, Wilco en ik bij jou thuis hebben mogen genieten. Ik koester onze vriendschap en onze "gezonde" passie voor de Bourgondische levensstijl. Ik wens je heel veel succes als leider van de nieuwe BMT-groep in Maastricht.

De leden van de beoordelingscommissie wil ik graag hartelijk bedanken voor de kritische beoordeling van mijn proefschrift. Prof. dr. Hans-Peter BrunnerLa Rocca, bedankt ook voor het vervullen van de voorzittersrol. Prof. dr. Robert Naeije en Prof. dr. Luc Snoeckx, ik ben mij ervan bewust dat dit proefschrift een nogal onconventionele modelmatige aanpak beschrijft van rechter hartfalen. Ik wil jullie daarom extra bedanken voor de kritische beoordeling van dit werk. Prof. dr. ir. van de Vosse, beste Frans, uiteraard wil ik ook jou ontzettend bedanken voor de kritische beoordeling van dit proefschrift, maar ook voor het feit dat je mij als BMT-student op de hoogte hebt gebracht van het cardiologische MRI-onderzoek van Tammo in Maastricht. Dear Prof. John Tyberg, thank you very much for critically assessing my thesis and for your willingness to participate in my public defense.

Beste Wilco, samen met Tammo en Theo vormden wij lange tijd de "TNT modeling agency". Bedankt voor deze mooie tijd! Op het zogenaamde Testosteron-lab hebben wij samen ongelofelijk veel lol gehad en geweldig genoten! Met je ongezouten humor en je enorme kennis van mechanica en Matlab heb je mij vaak verder geholpen met mijn onderzoek. Ons dagelijks "teske cola" was elke keer weer een welkome afleiding van ons serieuze modellenwerk. Ik ben blij dat wij de afgelopen jaren vrienden zijn geworden. Ook mijn voormalige directe collega's binnen de hartmechanicagroep, Jos Boeije en Borut Kirn, wil ik ontzettend bedanken voor de gezellige tijd die wij samen gehad hebben. Dear Borut, thank you very much for the sometimes endless and heated ThreeFlap-discussions. I still have to visit you in Slovak......(oops!) Slovenia.

Mijn paranimfen, Remco Megens en Koen Reesink, wil ik bedanken voor onze vriendschap en voor het feit dat ze mijn paranimfen willen zijn. Remco, jou ken ik het langst van alle collega's. Vanaf het begin vormden wij een perfecte match. Jij hebt mij in moeilijke tijden altijd moed ingesproken door alles te relativeren. Aangezien jij mij de compensatietheorie hebt geleerd, maak ik me momenteel een beetje zorgen om het feit dat je auto en huis steeds groter worden. Koen, wat voor Remco geldt, geldt ook voor jou. Wij voelen elkaar moeiteloos aan en kunnen elkaars gedachten lezen. Je peptalks hebben mij altijd weer positief laten denken. Ik heb intens genoten van onze lange discussies over persoonlijke en werkgerelateerde zaken en over vlinders en 
modellen die ergens op moeten lijken. Remco en Koen, jullie zijn beide uit het juiste hout gesneden en ik ben dan ook erg blij dat wij goede vrienden zijn geworden. Ik vind het heel erg (fijn) dat jullie mijn parapimps zijn.

Alle huidige en voormalige collega's van Biofysica, Fysiologie en de nieuwe BMT-groep wil ik bedanken voor de gezelligheid en de prettige werksfeer. Jullie hebben er allemaal voor gezorgd dat ik na een vakantie altijd weer ontzettend veel zin had om te gaan werken. Een speciaal woord van dank wil ik richten tot Prof. dr. Frits Prinzen. Beste Frits, jouw enthousiasme voor ons modellenwerk heeft mij vaak gestimuleerd (te simuleren); ontzettend bedankt voor je kritische commentaar op het pacingverhaal.

Alle collega's van de multidisciplinaire PAH werkgroep van het MUMC wil ik hartelijk bedanken voor hun hulp, gezelligheid en vooral enthousiasme. In het bijzonder wil ik dr. Karin Boomars, Bernard Broers en dr. Pieter van Paassen bedanken voor de praktische en inhoudelijke bijdragen aan mijn werk. Het wordt tijd voor een nieuwe PAH-BBQ!

Ik wil ook mijn collega-onderzoekers van het VUmc in Amsterdam, dr. J. Tim Marcus, dr. Anton Vonk Noordegraaf en Prof. dr. Nico Westerhof, ontzettend bedanken voor hun kritische blik op mijn werk en voor de samenwerking.

Dear Prof. Daniel G. Blanchard and Dr. Ehtisham Mahmud, I sincerely thank you for your interest in our work. Your scientific contribution to this thesis and your willingness to cooperate with us are gratefully appreciated.

Verder wil ik graag mijn vrienden van de G-Squad en van GVCG bedanken. Zodra ik in de auto naar Guttecoven zit, kan ik mijn werk altijd vergeten en ben ik gewoon Luumes. Dit is mij heel veel waard! Ik hoop dat jullie nu een klein beetje begrijpen waar ik me de afgelopen jaren mee bezig heb gehouden. Tevens wil ik mijn studievriend Remo Strangi bedanken. Pemo, zonder jou zou mijn tijd als BMT-student veel minder enerverend zijn geweest!

Lieve pap en mam, hoe kan ik jullie bedanken voor jullie vertrouwen in mijn kunnen? Jullie hebben mij altijd vrij gelaten in mijn keuzes. Dit waardeer ik enorm! Zonder jullie steun en goede zorgen was ik nooit zo ver gekomen. Bedankt voor alles! Aniek en Sander, ik wil ook jullie graag bedanken voor jullie betrokkenheid en voor het feit dat ik bij jullie altijd welkom ben. Ik hoop een goede "suikeroom" te worden voor de kleine!

Lieve Irene, zonder jou was dit proefschrift nooit op tijd klaar geweest. De onvoorwaardelijke steun en liefde die ik van jou gekregen heb, is onbetaalbaar. Samen kunnen wij enorm van het leven en van elkaar genieten. Ik heb het getroffen met jou! 

Curriculum Vitae 
Curriculum Vitae 


\section{JOZEF ELISABETH LUMENS}

was born on May 28, 1982, in Sittard, The Netherlands.

From 1994 until 2000, he attended secondary education at Trevianum in Sittard where he obtained his Gymnasium ( $\beta$ ) diploma. In 2000, he started his academic education at Eindhoven University of Technology where he obtained his Bachelor of Science degree (BSc) in Biomedical Engineering in September 2003 and his Master of Science degree (MSC) in Medical Engineering with the designation 'With Great Appreciation' in November 2005. During the Master Program, he did an internship at the Bioengineering Institute of the University of Auckland in New Zealand (January - April 2005) under the supervision of Assoc. Prof. Alistair A. Young. Furthermore, he did his final Master project, entitled "Effect of Aging on Left Ventricular Mechanics", at the Department of Biophysics at the Cardiovascular Research Institute Maastricht (CARIM) of Maastricht University under the supervision of Prof. Theo Arts and Dr. Tammo Delhaas. From 2006 until 2010, he worked as a PhD candidate at the Departments of Physiology and Biophysics (currently Department of Biomedical Engineering) at Maastricht University, again under the supervision of Prof. Theo Arts and Dr. Tammo Delhaas. During this PhD project, he used a patient-specific modeling approach to study the mechanisms of right heart failure in patients with pulmonary arterial hypertension. As of March 2010, he is working as a Postdoctoral Research Fellow at the Department of Biomedical Engineering at Maastricht University. 

List of Publications 


\section{Peer-Reviewed Journal Articles}

Lumens J, Delhaas T, Arts T, Cowan BR, and Young AA. Impaired subendocardial contractile myofiber function in asymptomatic aged humans, as detected using MRI. Am J Physiol Heart Circ Physiol 291: H1573-1579, 2006.

Kerckhoffs RC, Lumens J, Vernooy K, Omens JH, Mulligan LJ, Delhaas T, Arts T, McCulloch AD, and Prinzen FW. Cardiac resynchronization: Insight from experimental and computational models. Prog Biophys Mol Biol 97: 543-561, 2008.

Lumens J, Delhaas T, Kirn B, and Arts T. Three-wall segment (TriSeg) model describing mechanics and hemodynamics of ventricular interaction. Ann Biomed Eng 37: 2234-2255, 2009.

Lumens J, Arts T, Broers B, Boomars KA, van Paassen P, Prinzen FW, and Delhaas $T$. Right ventricular free wall pacing improves cardiac pump function in severe pulmonary arterial hypertension: A computer simulation analysis. Am J Physiol Heart Circ Physiol 297: H2196-2205, 2009.

Lumens J, Blanchard DG, Arts T, Mahmud E, and Delhaas T. Left ventricular underfilling and not septal bulging dominates abnormal left ventricular hemodynamics in chronic thromboembolic pulmonary hypertension. Submitted.

Lumens J, Arts T, Marcus JT, Westerhof N, Vonk-Noordegraaf A, and Delhaas T. Patient-specific modeling for assessment of ventricular mechanical asynchrony in pulmonary arterial hypertension. Submitted.

\section{Peer-Reviewed Proceedings}

Lumens J, Arts T, and Delhaas T. Ventricular mechanical asynchrony in pulmonary arterial hypertension: A model study. In: Functional Imaging and Modeling of the Heart, edited by Ayache N, Delingette $\mathrm{H}$, and Sermesant M. Nice, France: Springer Berlin / Heidelberg, 2009, p. 249-257.

Lumens J, Delhaas T, Kirn B, and Arts T. Modeling ventricular interaction: A multiscale approach from sarcomere mechanics to cardiovascular system hemodynamics. Pac Symp Biocomput 378-389, 2008. 


\section{BOOK CHAPTERS}

Arts T, Lumens J, Kroon W, Donker DW, Prinzen FW, and Delhaas T. Patientspecific modeling of cardiovascular dynamics with a major role for adaptation. In: Technology-driven personalized medicine: patient-specific computing of cardiovascular function, edited by Kerckhoffs RC, In Press. 
Juf

"Wat wil je worden" vroeg de juf,

't was in de derde klas,

ik keek haar aan en wist het niet,

'k dacht dat ik al iets was.

Toon Hermans 